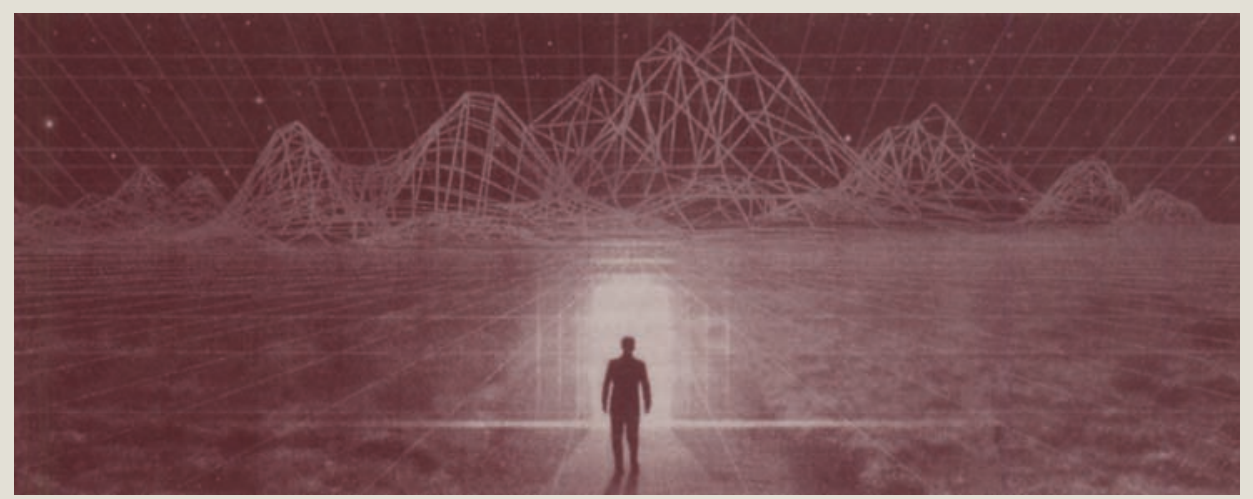

Alexandra Budke, Detlef Kanwischer, Andreas Pott (Hg.)

\title{
Internetgeographien
}

Beobachtungen zum Verhältnis

von Internet, Raum und Gesellschaft

Franz Steiner Verlag 
Internetgeographien 


\section{ERDKUNDLICHES WISSEN}

Schriftenreihe

für Forschung und Praxis

Begründet von

Emil Meynen

Herausgegeben

von Gerd Kohlhepp,

Adolf Leidlmair

und Fred Scholz

Band 136 


\section{Internetgeographien}

Beobachtungen zum Verhältnis von

Internet, Raum und Gesellschaft

Herausgegeben von

Alexandra Budke,

Detlef Kanwischer

und Andreas Pott 
Umschlagabbildung: BMG Video

Bibliographische Information der Deutschen Bibliothek

Die Deutsche Bibliothek verzeichnet diese

Publikation in der Deutschen Nationalbibliographie; detaillierte bibliographische Daten sind im Internet über < http://dnb.ddb.de> abrufbar.

ISBN 3-515-08506-8

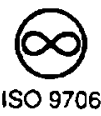

Jede Verwertung des Werkes außerhalb der Grenzen des Urheberrechtsgesetzes ist unzulässig und strafbar. Dies gilt insbesondere für Übersetzung, Nachdruck, Mikroverfilmung oder vergleichbare Verfahren sowie für die Speicherung in Datenverarbeitungsanlagen.

(C) 2004 by Franz Steiner Verlag Wiesbaden GmbH, Sitz Stuttgart. Gedruckt auf säurefreiem, alterungsbeständigem Papier.

Druck: Rheinhessische Druckwerkstätte, Alzey

Printed in Germany 


\section{STATT EINES VORWORTS}

\section{Geistesblitze}

21.07.01

Jena. Auf dem Gipfel des Jenzig

Alexandra: Wir müssen dringend etwas gemeinsam zum Thema Internet machen.

Andreas: $\quad$ Gute Idee. Virtualität und Raum ...

Detlef: $\quad$ Ich weiß nicht recht, immer diese Unterscheidung zwischen virtuellen und realen Welten. Das überzeugt mich nicht.

Andreas: $\quad \mathrm{Ja}$, du hast wohl recht. Vielleicht kann man es auch ganz anders machen.

\section{Brainstorming}

28.11.01 Versmold. Auf dem Bauernhof von Detlef

Alexandra: Lasst uns doch mal sammeln, was uns als „Raumwissenschaftler“ am Internet interessiert.

Detlef: $\quad$ Virtuelle Welten im Geographieunterricht. E-Learning und Raumgebundenheit ...

Andreas: $\quad$ Also mich interessieren Räume des Internets als soziale Konstruk-

tionen, das Verhältnis von Raum und Gesellschaft ...

Alexandra: Ich finde Internet als moderne Utopie und Globalisierung durch das Internet reizvoll ...

\section{Call for papers}

10.07.02 Frankfurt am Main. Uniturm, 29. Stock. Büro von Andreas

Andreas: $\quad$... der Begriff „Internetgeographien“ zeigt an, dass die Beobachtungsgebundenheit wissenschaftlicher Erkenntnis nicht invisibilisiert wird, sondern ...

Detlef: ? ? Hör auf mit diesem Soziologendeutsch!

Alexandra: Finde ich auch. Lasst uns den Satz umformulieren, dann sind wir mit dem Call for papers so gut wie fertig.

\section{Besprechung der Beiträge}

\subsubsection{Osnabrück, $30^{\circ} \mathrm{C}$. Wohnzimmer von Alexandra}

Alexandra: Grundsätzlich finde ich die Artikel gut. Aber wie gehen wir mit den unklaren Stellen um?

Detlef: $\quad$ Wir teilen uns die Autoren zur Beratung auf.

Andreas: $\quad \mathrm{Ja}$, und wir machen konkrete Vorschläge. 


\section{Vor der Drucklegung}

12.12.03 Versmold. Auf dem Bauernhof von Detlef

Detlef: Was müssen wir eigentlich dieses Wochenende machen?

Alexandra: Feiem?

Andreas: $\quad$ Klar, auch, wir müssen aber auf jeden Fall noch das Vorwort schreiben und uns ganz herzlich bei Sabine Seidel und Joachim Schindler für die Formatierung der Druckvorlage bedanken. Nicht zu vergessen sind auch Prof. Tilman Rhode-Jüchtern, Prof. Gerd Kohlhepp und Prof. Fred Scholz, die uns mit Rat und Tat unterstützt haben!

Alexandra: Und was machen wir als Nächstes?

Loxten / Osnabrück / Frankfurt am Main, im Januar 2004

Detlef Kanwischer, Alexandra Budke und Andreas Pott

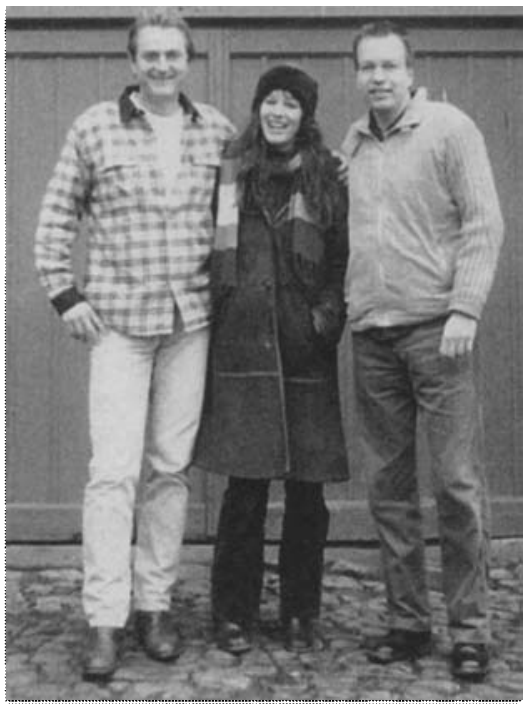




\section{INHALTSVERZEICHNIS}

Andreas Pott,

Alexandra Budke und

Detlef Kanwischer
Internet, Raum und Gesellschaft. Zur

$\underline{9}$

nisses

\section{Verortungen des Internets}

Niels Werber

Inga Heinze

Christian Langhagen-

Rohrbach

Holger Floeting
Von der Bagatellisierung des Raums. „Neue

Medien“ als „raumüberwindende Mächte“

Methoden und Anwendungsgebiete der Internetkartographie

Internet und Internet-User. Wer nutzt das Netz wo?

Internet und Stadt. Geht den Kommunen der Ort verloren?
23

41

57

79

\section{Der Geocode des Internets}

Christian Stegbauer

Cornelia Becker
Zum Verhältnis von Zentrum und Peripherie im Internet

Raum-Metaphern als Brücke zwischen Internetwahrnehmung und Internetkommunikation

\section{Räume des Internets}

Hubertus Niedermaier und Markus Schroer

Sabine Thabe und Arne Schlechter

Daniela Ahrens

Detlef Kanwischer
Sozialität im Cyberspace

Yonern als ironische Raum(de)-

143 konstruktion. Ethnographische Erkundungen in virtuellen Räumen

Internet, Nicht-Orte und die Mikrophysik des Ortes

163

Zwischen den Welten. Raumsimulationen im Netz 



\section{INTERNET, RAUM UND GESELLSCHAFT ZUR UNTERSUCHUNG EINES DYNAMISCHEN VERHÄLTNISSES}

Andreas Pott, Alexandra Budke und Detlef Kanwischer

Der vorliegende Band widmet sich dem Verhältnis von Internet, Raum und Gesellschaft. Das Forschungsfeld, das durch diese Begriffe konstituiert wird, lässt sich in vier Schritten bestimmen:

\section{INTERNET UND INTERNETFORSCHUNG}

Die Geschichte des Internets ist kurz, verblüffend kurz. Der erste technische Vorläufer des Kommunikationsmediums, das sich heute weltweit aus Alltag wie Wissenschaft nicht mehr wegdenken lässt, wurde in den 60er Jahren des letzten Jahrhunderts für das Militär der USA entwickelt. Dieses anfangs nur wenige Rechner verknüpfende dezentrale Netz, das sog. ARPANET, verbreitete sich in den 70er Jahren schnell im ganzen Land. Eine Reihe weiterer und zunehmend miteinander verknüpfter Computernetze kamen in den 80er Jahren dazu. Thre wachsende zivile Nutzung blieb jedoch noch weitgehend auf die US-amerikanischen Universitäten beschränkt. Die geradezu explosionsartige Verbreitung des uns heute bekannten Internets nahm erst vor einem Jahrzehnt seinen Anfang.

Vor gut zehn Jahren starteten drei Buchstaben ihren beeindruckenden Siegeszug: www - World Wide Web. Am 30. April 1993 gab das Direktorium des europäischen Kernforschungszentrums CERN (Conseil Européen pour la Recherche Nucléaire) das World Wide Web kostenlos für die Öffentlichkeit frei. Der britische Physiker Tim Berners-Lee hatte die Idee zu dem heute wichtigsten InternetDienst. Er wollte Wissenschaftlern des Genfer Instituts einen einfachen Austausch ihrer Daten unabhängig vom Computersystem oder Programm ermöglichen. Anfang der 90er Jahre entwickelte Berners-Lee das WWW sowie html (hypertext markup language), eine Programmiersprache, die eine einheitliche Formatierung von Dokumenten erlaubte. Diese können mit Hilfe eines Browsers betrachtet werden. Damit die Dateien via Internet übertragen werden können, bedarf es eines Protokolls, das die Übertragung regelt, das http (hypertext transfer protocol). Im Gegensatz zum WWW wurde mit dem Begriff Internet ursprünglich nur der physische Teil des weltweiten Netzwerks bezeichnet, d.h. seine aus untereinander verbundenen Rechnern bestehende technische Infrastruktur. Heute werden die Begriffe Internet und World Wide Web oft synonym verwendet.

Seit der Einführung des WWW hat sich das Phänomen Internet rasant ausgebreitet. In wenigen Jahren expandierte es nicht nur in praktisch alle Länder der Erde, sondern auch in nahezu alle Lebensbereiche. Mit der Verbreitung des Inter- 
nets und der Ausweitung seiner Nutzung nahm daher auch bald der Umfang der internetbezogenen Forschung zu. Entsprechend der Vielfalt der tangierten Bereiche handelt es sich hierbei um einen ausgesprochen interdisziplinären Forschungszusammenhang. So reicht das Spektrum der einschlägigen Publikationen von der Analyse technologischer und medienwissenschaftlicher Aspekte, sozialräumlicher Verteilungs- und Diffusionsprozesse sowie der Zugangsmöglichkeiten und Nutzungsweisen des Internets über seine Bedeutung für die Welt der Arbeit, der Wirtschaftsbeziehungen, der Politik oder der Bildung, die Entwicklungsmöglichkeiten von Städten oder Regionen bis zur Untersuchung der Veränderung von sozialen Ungleichheiten, kommunikativen Verhaltensweisen, Gruppen- und Identitätsbildungsprozessen oder Sexualität. ${ }^{1}$

Im Hinblick auf die gesellschaftliche Veränderungskraft des Internets schwingt in vielen der frühen Arbeiten der Internetforschung noch ein vergleichsweise optimistischer Grundton mit. Nicht selten klingt die Semantik an, die schon die Ausbreitung des Internets und seine wirtschaftliche und politische Förderung begleitet: Beim Internet handele es sich um ein fundamental neues, uneingeschränkt zu begrüßendes Phänomen, das einschneidende, ja umwälzende Veränderungen in Richtung einer besseren, demokratischeren, gerechteren Gesellschaft, einer von körperlichen Zwängen und räumlichen Beschränkungen befreiten Sozialität usw. zeitige. Mittlerweile ist dieser euphorische Ton der ,ersten Generation" der Internetforschung schwächer geworden. Seit einigen Jahren wird er abgelöst von einer distanzierteren, teilweise auch skeptischen Betrachtungsweise. Mit dem wachsenden zeitlichen Abstand zur Geburtsstunde des WWW lassen sich frühere Mutmaßungen und punktuelle Ersterhebungen über die Wirkungen des Internets durch systematischere Beobachtungen seiner Folgen ersetzen. Es eröffnet sich die Möglichkeit für umfassende und differenzierte empirische Analysen. Ebenso hilfreich ist heute die Fülle von Arbeitsgruppen, Forschungsnetzwerken, Tagungen, Zeitschriftenbeiträgen, Monographien und Sammelbänden, die der Erforschung des Internets gewidmet sind. Denn sie bieten neben ihren jeweiligen Ergebnissen oft wertvolles weiteres Beobachtungsmaterial. Sie ermöglichen es außerdem, nun den Blick auch auf die bisherige Internetforschung selbst zu richten und ihre Annahmen und Beobachtungsweisen kritisch zu rekonstruieren.

\section{INTERNET UND GESELLSCHAFT}

Den Einfluss, den das Internet auf die „Gesellschaft ausübt, kann man (noch) nicht vollständig überblicken und exakt messen. Aber die Vermutung ist nicht gewagt, dass er immens ist“" (Schmidt 2003). Große Worte wie das von der ,digitalen Revolution" nimmt zwar heute niemand mehr in den Mund, wenn vom Internet die Rede ist. Zu sehr ist die Nutzung des weltumspannenden Datennetzes Alltag geworden; als zu übertrieben haben sich viele Prophezeiungen erwiesen.

Vgl. etwa: Aoyama/Sheppard 2003, Becker/Paetau 1997, Bollmann/Heibach 1996, Dodge/Kitchin 2001, Faßler 1999, Funken/Löw 2003, Hülsmann 2000, Münker/Roesler 1997, Neverla 1998, Thimm 2000, Turkle 1998. 
Trotzdem scheint gerade im Rückblick die Bezeichnung „Revolution“ nicht ungerechtfertigt: Das Internet hat das Leben und Verhalten vieler Menschen im zurückliegenden Jahrzehnt wie kein anderes Medium seit der Erfindung des Fernsehens verändert. Die Art, wie sie sich informieren, wie sie miteinander kommunizieren und wie sie Geschäfte abwickeln, unterliegt im Zeitalter des Internets einem enormen Wandel.

In nur wenigen Jahren etwa hat sich das Internet vom neuen Informationsmedium zum komplexen Handelskanal entwickelt. Man kann heutzutage viele Bedarfsgüter per Internet kaufen. Umsatzstarke Märkte wie das Online-Auktionsgeschäft sind neu entstanden. Etablierte Anbieter in der Reisebranche, im Autohandel, im Musikgeschäft oder in den Banken haben sich auf neue Konkurrenten und Wettbewerbssituationen einstellen müssen. Billiganbieter haben sich eine starke Vertriebsplattform im Netz geschaffen und machen den alteingessenen Akteuren Konkurrenz. Als Reaktion gründen diese Online-Ableger, um auf ihre bisherigen teuren, personal- und mietintensiven Vertriebsorganisationen verzichten zu können. Auch Arbeitsabläufe werden durch die Verwendung des Internets verändert. In den virtuellen Büros transnationaler Konzerne beginnen deutsche Mitarbeiter ein Projekt, geben es abends an die Kollegen in den USA weiter und übernehmen die dort überarbeitete Fassung am nächsten Morgen wieder. Und in vielen Schulen und Universitäten gehört das internetbasierte, oft auch überregional vernetzte Lernen und Forschen mittlerweile zur alltäglichen Routine.

Die skizzierten Veränderungen zeigen, dass das Internet unter anderem die Globalisierung vorantreibt. Man kann im Internet gar eines der zentralen Medien der gegenwärtigen Globalisierungsprozesse sehen, das zwar neue soziale Ein- und Ausschlüsse hervorbringt, die Bedeutung der traditionellen nationalstaatlichen Ländergrenzen aber weiter verringert. Indem das Internet die Globalisierung von Wirtschaft, Wissenschaft, Kunst, Kultur, Medizin, Bildung oder Tourismus, aber auch von Terror-, Freundschafts- oder Migrationsnetzwerken oder neuen sozialen Protestbewegungen wie Attac stark befördert, trägt es entscheidend zur Entwicklung und Ausformung einer „Weltgesellschaft“" (Stichweh 2000) bei.

Die alltägliche Nutzung und Bedeutung des Internets hat noch weitere soziale Folgen. Das spielerische Experimentieren mit verschiedenen Identitäten in den Kommunikationsforen des Internets stellt die tradierten Identitätskonzepte und ihre Persistenz in Frage. Die bisherigen Grenzen zwischen Öffentlichkeit und Privatheit werden noch durchlässiger und verschieben sich. Und schon heute werden Kinder in der Schule zu Außenseitern, wenn sie zugeben müssen, zu Hause keinen Zugang zum Internet zu haben. Fast alle Studierenden sowie drei Viertel aller leitenden Angestellten und höheren Beamten in Deutschland surfen im Internet. Dagegen sind Arbeiter und einfache Angestellte sowie Rentner vergleichsweise selten im Netz unterwegs. Die Preis-, Informations- und Dienstleistungsvorteile des Internets sind ihnen verwehrt, sie bleiben den InternetNutzern vorbehalten. Soziologen sprechen von der digitalen Spaltung der Gesellschaft.

Nicht zuletzt verändert das Internet das Realitätsverständnis. Für diejenigen, die das Internet nutzen, treten neue Handlungs-, Erfahrungs- und Wirklichkeitsbe- 
reiche neben die bisher bekannten. Die sich online erschließende Welt des Internets, diese ,virtuelle Realität“ des Cyberspaces, ergänzt, überlagert und modifiziert die ,reale Welt".

\section{INTERNET UND RAUM}

Wie diese Welt- und Cyberspace-Begriffe sind auch viele andere Begriffe der Internetkommunikation räumlich konnotiert: Die Internetnutzer sind im weltumspannenden Netz auf Datenautobahnen unterwegs, sie surfen in den Tiefen und Weiten des Cyberspaces, diesem global village, tauschen sich in Chatrooms oder elektronischen Foren aus, kaufen auf virtuellen Marktplätzen, studieren fremde oder errichten eigene Homepages usw. Auch in der Internetforschung tauchen zur Beschreibung und Analyse der neuen Phänomene regelmäßig diese oder ähnliche Raumbegriffe und -metaphern auf.

Die auffallende Prominenz der räumlichen Semantik überrascht auf den ersten Blick, wird das Internet doch häufig gerade als Raumüberwinder oder Entgrenzungsmedium verstanden. Im Internet scheinen die Kommunikation und der Aufbau sozialer Beziehungen völlig unabhängig von räumlichen Grenzen zu erfolgen; noch müheloser als ältere Kommunikationsmittel scheint das Internet erdräumliche Entfernungen zu überwinden (vgl. Schroer 2001). Nicht nur die Netzpioniere und „Netzenthusiasten“ (ebd.), sondern auch manche Sozialwissenschaftler erwarten deshalb vom Internet die weitere Enträumlichung bzw. Entterritorialisierung der Kommunikation, den endgültigen ,death of distance“ (Cairncross 1997) und damit das Entstehen einer ,atopischen Gesellschaft" (Willke 2001) - worin im Übrigen auch ihre Hoffnung begründet liegt, das Internet helfe, bisherige soziale Ungleichheiten zu überwinden.

An dieser Sichtweise wird allerdings auch deutliche Kritik geübt. Die soziale Bedeutung von Räumlichkeit, Lokalität und Körperlichkeit werde durch das Internet nicht aufgehoben, sondern allenfalls modifiziert (vgl. z.B. Krämer 2002). Der angeblich durch das Internet vorangetriebene Bedeutungsverlust räumlicher Kategorien und Verteilungen wird unter anderem mit der Beobachtung in Frage gestellt, dass das Internet die (Welt-)Gesellschaft hinsichtlich der Internet-Nutzer, -produzenten und -infrastruktur auch geographisch spalte - z.B. entlang der regionalen Unterscheidungen Industrie-/Entwicklungsländer und Stadt/Land (vgl. Castells 2001). Ferner wird darauf hingewiesen, dass das Internet alles andere als ein raumloses und raumauflösendes Medium sei. Im Kontext der strategischen Interessen der Weltmacht USA lässt sich das Internet als geopolitische „Kommunikationswaffe“ deuten (vgl. Maresch 2002, S. 247ff.), als eine machtpolitisch wichtige, da herrschaftsausbauende und -sichernde, Technologie, die der „erdverbundenen" US-Politik den (Welt-)Raum erschließe (vgl. auch Maresch/Werber 2002, S. 19).

Die Folgen des Internets für die gesellschaftliche Relevanz des Raumes werden also konträr gedeutet. Augenscheinlich unterliegt dieser Auseinandersetzung aber ein Raumbegriff, der „Raum“ auf Erdraum, auf physisch-materielle bzw. 
körperlich-leibliche Aspekte reduziert. Demgegenüber ist die Raumbegrifflichkeit der Internetkommunikation anderer, nämlich kommunikativ-formaler Art. Die Funktionen der in dieser Kommunikation gebildeten räumlichen Formen für die Konstituierung, Strukturierung und Nutzung des Internets werden von der bisherigen Debatte zur Raumrelevanz fast vollständig ausgespart. Ebenso ungeklärt bleibt ihre Beziehung zu den in ihrer Bedeutung umstrittenen physisch-materiellen Räumen.

Insgesamt legen die genannten Beobachtungen die Vermutung nahe, dass das Internet das Verhältnis von Raum und Gesellschaft verändert. In welcher Weise dies jedoch geschieht, ist keineswegs klar. Offensichtlich bedarf es dazu auch einer Reflexion der in der wissenschaftlichen Beobachtung und Analyse verwendeten Raumkonzeptionen.

\section{RAUM UND GESELLSCHAFT}

Die Erforschung des Raum-Gesellschafts-Verhältnisses hat durch den Globalisierungsdiskurs inzwischen eine fachübergreifende Aufmerksamkeit erfahren. Auf die längste Beschäftigung und größte Erfahrung mit dieser Problematik kann sicherlich die Geographie zurückblicken. Ist man mit ihren fachkonstitutiven Problemstellungen vertraut, erinnert auch die für viele Arbeiten der Internetforschung bindende Leitunterscheidung zwischen wirklicher und virtueller Realität (bzw. Welt) an Wohlbekanntes. Sie lässt verblüffende Parallelen zu der vor allem in der Sozialgeographie schon lange diskutierten Unterscheidung von physischem Raum (Umwelt, Materie, Verteilungen von Menschen und Objekten auf der Erdoberfläche usw.) und sozial konstituiertem Raum (durch Wahrnehmung, Handlung und Kommunikation) erkennen (vgl. zuletzt Hard 2002, Meusburger 1999, Miggelbrink 2002a oder Werlen 2000).

Seit einigen Jahren entdecken auch die Soziologie und andere Sozial- und Kulturwissenschaften die Raum-Kategorie wieder, nachdem ihr dort jahrzehntelang nur ein Schattendasein beschieden war. Das neue Interesse am Raum und der sozialen Relevanz räumlicher Unterscheidungen motiviert verschiedene Versuche einer angemessenen Konzeptualisierung von Raum im Rahmen aktueller Gesellschaftstheorien (vgl. exemplarisch Kuhm 2000 und Löw 2001).

Vergleicht man die sozialgeographische Raum-Debatte der letzten Jahre mit der soziologischen, zeigen sich viele Gemeinsamkeiten. Fachübergreifend werden mit „Raum“ entweder physisch-materielle Phänomene bezeichnet, die der Umwelt der Gesellschaft angehören, oder hybride Phänomene, in denen sich Physisches und Soziales mischt (z.B. „Sozialräume"), oder aber ausschließlich sozial konstituierte Sinnphänomene. Während die ersten beiden Sichtweisen im Allgemeinen mit einem Behälter- und/oder einem relationalen Raumverständnis korrespondieren, kommen konstruktivistische bzw. akteurs-, handlungs-, kommunikations- und diskurstheoretische Ansätze zwar teilweise auch in der zweiten, insbesondere aber in der dritten Sichtweise vor. 
Neben diesen generellen Gemeinsamkeiten findet man allerdings auch bedeutsame Unterschiede. So lässt sich eine interessante diskursive Gegenbewegung beobachten, die zumindest für Teilbereiche der sozialgeographischen und soziologischen Debatten gilt. Im Gegensatz zu der die geographische Entwicklung schon seit längerem prägenden und zunehmenden Versozialwissenschaftlichung des Faches führt die Wieder- bzw. Neuentdeckung des Raumes in der Soziologie zu einer schon fast traditionell geographisch zu nennenden Zentralstellung eines immer auch materiell gedachten Raumbegriffs. Für viele Teilbereiche der Geographie lässt sich beobachten, wie sie sich allmählich, aber kontinuierlich von einem dinglich-materiell geprägten Raumverständnis entfernen. Stattdessen rücken sie vermehrt die weiteren sozialen Kontexte und Praktiken in der modernen Gesellschaft, in denen dann unter anderem auch räumliche Unterscheidungen relevant (gemacht) werden, in ihren Untersuchungsfokus. Dagegen laufen Teile der soziologischen Diskussion durch ihre gegenwärtige Zentrierung auf die konstitutive Bedeutung, die sie der Raum-Kategorie für die Funktionsweise und das Verständnis der modernen Gesellschaft zuschreiben, Gefahr, die aus der geographischen Argumentation inzwischen weitgehend verschwundenen Fallen des Geo- und Raumdeterminismus emeut aufzustellen.

Ungeachtet dieser Unterschiede gehört zu den wesentlichen Erkenntnissen der jüngeren Raum-Diskussion in den Sozialwissenschaften, dass Räume keine vorgegebenen oder konstanten Handlungsrahmen, in denen sich Soziales abspielt, sondern spezifische soziale Herstellungsleistungen sind. Räume werden erst durch die Verwendung räumlicher Unterscheidungen wie nah/fern, hier/da, oben/unten usw. im und durch den Handlungs- bzw. Kommunikationsprozess hervorgebracht und sozial relevant. Am konsequentesten lässt sich dieses Argument mit Hilfe konstruktivistischer bzw. beobachtungstheoretischer Ansätze entwickeln (vgl. Miggelbrink 2002b), also zum Beispiel im Rahmen der Luhmann'schen Systemtheorie. Man muss diesen Ansätzen (und ihrer vollständigen Dematerialisierung des Raumbegriffs) nicht folgen, um eine weitere Einsicht zu teilen, die sich für die systematische Untersuchung von „Raumfragen“ (Hard 1999) als überaus nützlich und fruchtbar erweist: Geht man davon aus, dass Räume (unabhängig davon, ob als materielle und/oder soziale gedacht) nicht immer schon vorhanden sind, sondern erst im Handlungs- bzw. Kommunikationsprozess „konstruiert“ werden und daher ständig ,in Bewegung“" sind (vgl. Schroer 2003), dann gilt es, genau diesen Herstellungs- und Veränderungsprozess samt seiner sozialen Kontextbedingungen zu untersuchen, um die Relevanz von Raum und räumlichen Schemata rekonstruieren zu können. Mit anderen Worten: In der sozialwissenschaftlichen Analyse sind nicht Handlungen und Kommunikationen, soziale Beziehungen o. A. im Raum, sondern genau umgekehrt: Raum und Raumunterscheidungen in den Handlungen, Kommunikationen, Beschreibungen, Beziehungen o. ̈. zu untersuchen.

Daraus wiederum folgt die methodologische Konsequenz, die Untersuchung raumbezogener Fragestellungen als Raum-Beobachtungen zu konzipieren und verschiedene Beobachtungspositionen zu unterscheiden: Der (die) Handelnde (also z.B. der surfende Internetnutzer, aber auch der Internetforscher), der die Welt (d.h. „seine“ Realität) mit räumlichen Schemata beobachtet (konstruiert) 
bzw. in seinen Handlungen und Kommunikationen mit Hilfe räumlicher Unterscheidungen spezifische Raumformen beschreibt oder hervorbringt, ist ein RaumBeobachter (oder Raum-Konstrukteur) erster Ordnung. Derjenige, der diesen Beobachter erster Ordnung daraufhin beobachtet, wie (und wozu) dieser Räume konstruiert, ist ein Raum-Beobachter zweiter Ordnung. Usw. (Das gleiche Argument gilt natürlich entsprechend für Gruppen, Organisationen, soziale Kommunikationssysteme u. Ä.)

\section{FRAGEN UND BEOBACHTUNGEN}

Entwickelt man den Untersuchungsgegenstand des Buches auf diese Weise, sieht man, dass es sich bei dem Verhältnis von Internet, Raum und Gesellschaft um ein in mehrfacher Hinsicht dynamisches und ungeklärtes Verhältnis handelt. Die offenen Forschungsfragen lassen sich zu fünf Fragenkomplexen bündeln, zu deren Beantwortung der Band beitragen will:

\section{1) Räumliche Formen und Internet:}

Welche Rolle spielen räumliche Unterscheidungen für den Strukturaufbau und die Nutzung des Internets? Warum werden das Internet und die mit ihm verbundenen Phänomene sowohl im nichtwissenschaftlichen Alltag als auch in der Wissenschaft regelmäßig mit Raumbegriffen beschrieben?

2) Internet als Raum:

Inwiefern lässt sich das Internet als Raum interpretieren? Welche Räume entstehen im Internet und wie werden sie konstruiert? Bewirken sie neue Formen der Raumerfahrung und des Raumerlebens? Welche Funktionen erfüllen sie für Anbieter wie für Nutzer?

3) Internet und physischer Raum:

Wie lässt sich das Verhältnis von Internet und physischem Raum deuten? Welche Relevanz haben materielle Bestandteile des Internets, ihre erdoberflächliche Verteilung sowie die Verteilung der Internetnutzer? Entwertet das Internet die gesellschaftliche Bedeutung des physischen Raumes und erdräumlicher Distanzen?

4) Gesellschaftliche Veränderungspotenziale des Internets:

Inwiefern bergen das Internet und die mit ihm hervorgebrachten Räume Potenziale für gesellschaftliche Innovationen? Tragen sie zur Entstehung einer „besseren Welt" bei? Fördern sie Demokratisierung und den Abbau sozialer und wirtschaftlicher Disparitäten?

5) Internet und Raumkonzeptionen:

Inwiefern bestätigt, unterläuft oder verändert das Internet die alltagsweltliche Vorstellung eines behälterförmigen, materiellen und die Subjekte umgebenden Raumes? Wie lässt sich das Internet als Forschungsobjekt nutzen, um der sozialwissenschaftlichen Debatte zum Verhältnis von Raum und Gesellschaft neue Impulse zu verleihen? 
Die fünf Fragenkomplexe verdeutlichen ebenso wie die obigen Ausführungen zum Raumbegriff den besonderen "räumlichen" Akzent der in diesem Band versammelten sozialgeographischen und soziologischen Untersuchungen. Die Einsichten und Problemstellungen der jüngeren interdisziplinären Raum-Diskussion sollen hier für die Untersuchung des Internets und seiner gesellschaftlichen Folgen fruchtbar gemacht werden. Daraus resultiert nicht zuletzt die beobachtungstheoretische Ausrichtung des Bandes. Vor dem Hintergrund der beschriebenen Konsequenzen, die sich für raumbezogene Untersuchungen aus der theoretischen Diskussion ergeben, lassen sich die Beiträge als Raum-Beobachtungen erster, zweiter und dritter Ordnung lesen - mit einem Wort: als Internetgeographien.

Der zusammenfassende Begriff Internetgeographien (Plural!) soll folglich nicht zum Ausdruck bringen, dass mit dem Band der Versuch der Begründung einer weiteren geographischen Bindestrich-Disziplin, i.e. einer Internetgeographie (Singular!), unternommen wird. Der Begriff wird nicht in einem disziplinärprogrammatischen Sinne verwendet. Vielmehr ist er in einem raum- und beobachtungstheoretischen Sinne zu verstehen: Die in Frage stehende Beziehung von Internet, Raum und Gesellschaft wird im vorliegenden Band aus verschiedenen Perspektiven untersucht, ${ }^{2}$ die sich gerade darin gleichen, dass sie alle spezifische Formen von Raum-Beobachtungen bzw. Raum-Beschreibungen darstellen. Im übertragenen Sinne kann auch der Band als ganzer als eine Internetgeographie verstanden werden. Denn durch die Auswahl der Artikel und damit Perspektiven bemüht er sich, die „Landschaft“ der Forschung nachzuzeichnen. Insofern bezeichnet der Begriff Internetgeographien die Einheit des Bandes und die konzeptionelle Gemeinsamkeit der Beiträge. Er zeigt an, dass die Beobachtungsgebundenheit wissenschaftlicher Erkenntnis ernst genommen wird und systematisch für die Analyse und ihre Reflexion genutzt werden soll:

Raum-Beobachtungen erster Ordnung (RB1.O) finden sich in den Beiträgen, in denen die wissenschaftlichen Beobachter die "Geographie des Internets“ beschreiben, also das Internet, das man auch anders beschreiben könnte, in ihren Beschreibungen räumlich interpretieren, räumliche Muster aufzeigen, usw.

Raum-Beobachtungen zweiter Ordnung (RB2.O) kennzeichnen zum einen die Beiträge, in denen die wissenschaftlichen Beobachter die „Geographien der Internet-Nutzer" beschreiben, also beobachten, ob, wie und wozu die Nutzer räumliche Unterscheidungen verwenden und Räume produzieren (RB2.Oa). Zum anderen handelt es sich um Raum-Beobachtungen zweiter Ordnung, wenn die „Geographien der (Internet-)Forschung" Thema sind, wenn also die Forschung selbst daraufhin beobachtet wird, ob und wie sie das Internet (bzw. die Welt) räumlich interpretiert (RB2.Ob).

Und um Raum-Beobachtungen dritter Ordnung handelt es sich entweder, wenn beobachtet wird, wie die Nutzer Räume der (Internet-)Kommunikation oder die in Softwareprogrammen präsentierten Räume selbst räumlich interpretieren (RB3.Oa), oder wenn schon die beobachtete Forschung das Internet, die Internet-

2 Beziehungsweise - strikt beobachtungstheoretisch gesprochen - als Forschungsgegenstand multiperspektivisch konstituiert. 
kommunikation oder andere soziale Zusammenhänge auf das Vorkommen und die Relevanz von Räumen hin untersucht (RB3.Ob).

Die Mehrheit der Autorinnen und Autoren nimmt im Gang ihrer Argumentation allerdings nicht nur eine, sondern - nacheinander oder im Wechsel - zwei (in einem Fall auch vier) der verschiedenen Beobachtungspositionen ein. Daher ist die beobachtungstheoretische Unterscheidung für den Vergleich der einzelnen Beiträge weniger nützlich als für die Reflexion ihrer jeweiligen Argumentationslogik.

Ähnlich wie im Falle der Beobachtungspositionen bearbeiten alle Autorinnen und Autoren mehrere der genannten fünf Fragenkomplexe. Somit stellen die mit der beobachtungstheoretischen Unterscheidung und den Fragenkomplexen markierten Perspektiven zwar zwei unterschiedliche, dem Band innewohnende Strukturprinzipien dar. Sie eignen sich aber nicht für seine Gliederung. Diese folgt einer anderen systematischen Differenzierung. Bei den nachfolgenden Internetgeographien stehen erstens die Verortungen des Internets im Vordergrund, für die insbesondere (aber bei weitem nicht nur) physische Aspekte berücksichtigt werden; zweitens zielt die Analyse auf den (abstrakten) strukturgenerierenden ,räumlichen" Geocode des Internets; und drittens liegt der Untersuchungsschwerpunkt auf (ausschließlich) kommunikativ konstituierten Räumen des Internets:

I) Der erste Teil des Bandes behandelt aus verschiedenen Perspektiven die Verortungen des Internets. Der Beobachtungsfokus wird hierbei auf physische Aspekte des Internetmediums, seiner Nutzung und der Raum-Kategorie gerichtet. Niels Werber rekonstruiert die blinden Flecken der Medien- und der soziologischen Systemtheorie in Bezug auf Raum, Materialität und Macht (RB2.Ob und RB3. $\mathrm{Ob}$ ). Aus seiner Kritik ihrer Raumlosigkeit und Bagatellisierung des territorialen Standortes leitet er die Forderung einer auch physisch-erdräumlich und geopolitisch informierten Soziologie ab. Dazu bedarf es unter anderem des Wissens um die erdräumliche Verteilung der Internetinfrastruktur und ihrer Nutzer. Wie man diese Verteilung beobachten und für die sozialwissenschaftliche Analyse fruchtbar machen kann, zeigt Inga Heinze, indem sie die verschiedenen Typen und Anwendungsgebiete der Internetkartographie vorstellt (RB2.Ob). Als empirischer Anwender dieser Methode untersucht Christian Langhagen-Rohrbach die regionalen Verteilungsmuster der Internetinfrastruktur und ihrer Nutzer in Deutschland sowie deren soziodemographische Struktur (RB1.O und RB2.Ob). Er weist exemplarisch nach, dass das Internet bestehende sozialräumliche Ungleichheitsstrukturen reproduziert und weiter festigt, und kommt zu dem Ergebnis, dass das Internet im zunehmenden globalen Wettbewerb insbesondere dem Bedeutungserhalt der Städte und Metropolen nützt. Wie diese vor dem Hintergrund ihrer bisherigen Erfahrungen mit älteren Kommunikations- und Informationstechnologien auf das Internet reagieren, es in ihren kommunalpolitischen Bezugsrahmen integrieren und internetbezogene Entwicklungsstrategien verfolgen, ist Gegenstand des Beitrags von Holger Floeting (RB1.O und RB2.Oa). 
II) Der zweite Teil des Bandes identifiziert auf zweierlei Weise einen strukturgenerierenden Geocode des Internets. Inspiriert von dem Prinzip der Selbstähnlichkeit sozialer Strukturen untersucht Christian Stegbauer an den Beispielen sozialer Beziehungen Einzelner, internetbasierter Kommunikationsforen sowie der Verlinkungsstruktur des gesamten WWW, inwiefern das Internet auf verschiedenen Aggregationsebenen stets dem gleichen ,räumlichen“ Strukturprinzip, i.e. einem Zentrum-Peripherie-Muster, folgt (RB1.O und RB2.Oa). Dagegen geht Cornelia Becker in ihren wahrnehmungstheoretischen Überlegungen und einem empirischen Anwendungsbeispiel der Funktion der für die Internetwahrnehmung und kommunikation so prominenten Raum-Metaphern nach (RB2.Oa).

III) Der eingehenden Bestimmung und Untersuchung verschiedenartiger Räume des Internets widmet sich der dritte Teil des Bandes. Hubertus Niedermaier und Markus Schroer charakterisieren den Cyberspace als ort- und bodenlosen Raum ohne physische Ausdehnung und fragen nach den Merkmalen der Sozialität, die dieser sozial konstituierte Raum ermöglicht. Dazu diskutieren sie, inwiefern der Cyberspace eine fragmentierte Öffentlichkeit beherbergt und außerdem auch selbst als Interaktionspartner auftritt - und welche Folgen dies für das soziologische Verständnis von Raum, Interaktion und Kommunikation hat (RB1.O und RB2.Oa). Auf der Basis einer mehrmonatigen teilnehmenden Beobachtung unternehmen Sabine Thabe und Arne Schlechter eine sprachphilosophisch und kulturtheoretisch angeleitete Ethno- und Topographie eines konkreten Kommunikationsforums im Internet. Aus ihrer exemplarischen Analyse der Strukturen und Logiken virtueller Gemeinschaften leiten sie weitreichende theoretische und methodologische Schlüsse für die zukünftige sozialwissenschaftliche Untersuchung von Raum(de)konstruktionen ab (RB1.O, RB2.Oa, RB3.Oa und RB3.Ob). Daniela Ahrens entfaltet die These, dass die mit dem Internet fortschreitende Enträumlichung der Kommunikation als Voraussetzung für die soziale Relevanz fungiert, die räumlichen Unterscheidungen bei der Bearbeitung von Komplexität zukommt. Dazu diskutiert sie Faßlers Raumtypologie und Augés Konzept der Nicht-Orte im Hinblick auf ihre Deutungskraft für das Verhältnis von Internet und Raum. In ihrer kritischen Auseinandersetzung mit diesen Ansätzen zeigt sie, wie deren Schwächen mit Hilfe des für die Analyse des Internet sehr fruchtbaren relationalen Raumverständnisses überwunden werden können (RB2.Ob und RB3.Ob). Den dritten Teil des Buches beschließt der Beitrag von Detlef Kanwischer. Am Beispiel eines netzgestützten Simulationsspiels für den Geographieunterricht zeigt er auf, mit welchen Raumkonzeptionen derartige Spiele arbeiten und welchen Einfluss sie auf die Wirklichkeits- und Raumvorstellungen von Schüler(inne)n haben können. Auf der Grundlage des Medienwirkungsmodells von Fritz und des mit seiner Hilfe analysierten Interviews mit einer Spielerin werden das Verstrickungsverhältnis zwischen virtueller und realer Welt sowie der Einsatz von Simulationsspielen im Unterricht diskutiert (RB2.Ob und RB3.Oa). 


\section{LITERATUR}

Aoyama, Yuko und Eric Sheppard (2003): The Dialectics of Geographic and Virtual Space. Guest Editorial, in: Environment and Planning A, Vol. 35, No. 7, S. 1151-1156.

Becker, Barbara und Michael Paetau (Hrsg.) (1997): Virtualisierung des Sozialen. Die Informationsgesellschaft zwischen Fragmentierung und Globalisierung, Frankfurt a.M.

Bollmann, Stefan und Christine Heibach (Hrsg.) (1996): Kursbuch Internet. Anschlüsse an Wirtschaft und Politik, Wissenschaft und Kultur, Mannheim.

Caimcross, Frances (1997): The Death of Distance. How the Communications Revolution will Change our Lives, Harvard.

Castells, Manuel (2001): The Internet Galaxy: Reflections on the Internet, Business, and Society, Oxford.

Dodge, Martin und Rob Kitchin (2001): Atlas of Cyberspace, Harlow.

Faßler, Manfred (Hrsg.) (1999): Alle möglichen Welten: Virtuelle Realität - Wahmehmung Ethik der Kommunikation, München.

Funken, Christiane und Martina Löw (Hrsg.) (2003): Raum - Zeit - Medialität. Interdisziplinäre Studien zu neuen Kommunikationstechnologien, Opladen.

Hard, Gerhard (1999): Raumfragen, in: Meusburger, Peter (Hrsg.): Handlungszentrierte Sozialgeographie, Erdkundliches Wissen 130, Stuttgart, S. 133-162.

Hard, Gerhard (2002): Landschaft und Raum. Aufsätze zur Theorie der Geographie. Band 1, Osnabrücker Studien zur Geographie 22, Osnabrück.

Hülsmann, Thorsten (2000): Geographien des Cyberspace, Wahrnehmungsgeographische Studien 19 , Oldenburg.

Krämer, Sybille (2002): Verschwindet der Körper? Ein Kommentar zu virtuellen Räumen, in: Maresch, Rudolf und Niels Werber (Hrsg.): Raum - Wissen - Macht, Frankfurt a.M., S. 49-68.

Kuhm, Klaus (2000): Raum als Medium gesellschaftlicher Kommunikation, in: Soziale Systeme. Zeitschrift für soziologische Theorie, Jg. 6, Heft 2, S. 321-348.

Löw, Martina (2001): Raumsoziologie, Frankfurt a.M.

Maresch, Rudolf (2002): Hard Power/Soft Power. Amerikas Waffen globaler Raumnahme, in: Maresch, Rudolf und Niels Werber (Hrsg.): Raum - Wissen - Macht, Frankfurt a.M., S. 237-262.

Maresch, Rudolf und Niels Werber (2002): Permanenzen des Raums, in: dies. (Hrsg.): Raum Wissen - Macht, Frankfurt a.M., S. 7-30.

Meusburger, Peter (Hrsg.) (1999): Handlungszentrierte Sozialgeographie. Benno Werlens Entwurf in kritischer Diskussion, Erdkundliches Wissen 130, Stuttgart.

Miggelbrink, Judith (2002a): Der gezähmte Blick. Zum Wandel des Diskurses über „Raum“ und „Region“ in humangeographischen Forschungsansätzen des ausgehenden 20. Jahrhunderts, Beiträge zur Regionalen Geographie 55, Leipzig.

Miggelbrink, Judith (2002b): Konstruktivismus? „Use with Caution“ ... Zum Raum als Medium der Konstruktion gesellschaftlicher Wirklichkeit, in: Erdkunde, Band 56, Heft 4, S. 337350.

Münker, Stefan und Alexander Roesler (Hrsg.) (1997): Mythos Internet, Frankfurt a.M.

Neverla, Irene (Hrsg.) (1998): Das Netz-Medium. Kommunikationswissenschaftliche Aspekte eines Mediums in Entwicklung, Opladen. 
Schmidt, Holger (2003): Das digitale Zeitaiter, in: Frankfurter Allgemeine Zeitung, 26. Juli 2003, S. 1.

Schroer, Markus (2001): Land und (Daten-)Meer. Zur Besetzung von Răumen im Internet, in: Telepolis. Magazin der Netzkultur, 27.8.2001, online: www.telepolis.de

Schroer, Markus (2003): Raumgrenzen in Bewegung. Zur Interpretation realer und virtueller Räume, in: Funken, Christiane und Martina Löw (Hrsg.): Raum - Zeit - Medialităt. Interdisziplinäre Studien zu neuen Kommunikationstechnologien, Opladen, S. 217-236.

Stichweh, Rudolf (2000): Die Weltgesellschaft. Soziologische Analysen, Frankfurt a.M.

Thimm, Caja (Hrsg.) (2000): Soziales im Netz: Sprache, Beziehungen und Kommunikationskulturen im Internet, Opladen.

Turkle, Sherry (1998): Leben im Netz. Identităt im Zeichen des Internet, Reinbek.

Werlen, Benno (2000): Sozialgeographie. Eine Einführung, Bern u.a.

Willke, Helmut (2001): Atopia. Studien zur atopischen Gesellschaft, Frankfurt a.M. 


\section{Verortungen des Internets}





\title{
VON DER BAGATELLISIERUNG DES RAUMS \\ "NEUE MEDIEN" ALS »RAUMÜBERWINDENDE MÄCHTE»
}

\author{
Niels Werber
}

\section{RAUM, TECHNIK, GESELLSCHAFT - MEDIEN DER GEOGRAPHIE UND DER GEOPOLITIK}

\begin{abstract}
„Auf die längste Beschäftigung und größte Erfahrung mit der Raum-Gesellschafts-Thematik kann sicherlich die Geographie zurückblicken."1 Die Annahme, dass Gesellschaften in einer spezifischen geographischen Nische evoluieren, wird bereits von Herder und Hegel vertreten und seit der Mitte des 19. Jahrhunderts von der jungen Disziplin der Geographie erforscht. Der Begriff „Kulturlandschaft" bezeugt diesen Zusammenhang. „Ein Blick auf die Landkarte“, empfiehlt der Hegelianer Gotthard Günther allen Historikern, sei „sehr belehrend.“2 Schnelle und evidente politische Einsicht verspricht noch Noam Chomsky seinen Lesern „by looking at a map“. ${ }^{3}$ Friedrich Ratzel, der Zoologe, Mentor der deutschen Geopolitik und Promotor der „Anthropogeographie“, hat mit Rückgriff auf Herder seit den 1880er Jahren immer wieder die These vertreten, alle Kultur- und Geschichtswissenschaft brauche „die Geographie, um den geschichtlichen Boden und die politischen Raumgebilde zu zeichnen, zu messen und zu beschreiben." ${ }^{* 4}$ Niemand verstehe die politische Geschichte der Nationen, wer die ,geographische Lage“ ihrer Staaten ignoriere; viele Eigentümlichkeiten etwa Britanniens seien auf die Insellage oder Deutschlands auf die berüchtigte „Mittellage“ zurückzuführen. ${ }^{5}$ Dies sehen selbst nach dem zweiten Weltkrieg auch us-amerikanische Geopolitiker noch so. 6

Die konkrete Ausprägung der Wechselbeziehung von Raum und Gesellschaft hängt aus Ratzels Sicht vom Stand der Verkehrstechniken ab. Erst eine Flotte macht die USA zum Nachbarn Japans, erst die Eisenbahn macht Russland zu
\end{abstract}

Budke/Kanwischer/Pott (2002): Internetgeographien. Call for Papers, S. 2.

Günther 2000, S. 94.

Chomsky 2002, S. 112.

Ratzel: „Mensch und Erde“, in: Haushofer (Hrsg.) (1991): Erdenmacht und Völkerschicksal, Stuttgart: „Weist man der Geschichte das zeitliche Geschehen, der Geographie das räumliche Sein zur Erforschung zu, so vergesse man nicht: alles Geschehen findet im Raum statt, jede Geschichte hat also ihren Schauplatz. Herders Satz von der Geschichte als einer in Bewegung gesetzten Geographie bleibt wahr" (S. 9).

5 Vgl. Ratzel: „Gunst und Verhängnis der Lage“, in: Haushofer (Hrsg.) (1991): Erdenmacht und Völkerschicksal, Stuttgart, S. 132 u. 135.

6 Vgl. Walsh 1946. 
einem eurasischen Staat. ${ }^{7}$ Der „Verkehr“ zielt folglich auf die „Überwindung des Raums“ ${ }^{8}$ Ratzels Herausgeber Karl Haushofer (1934) hat in dieser Tradition von „raumüberwindenden Mächten“ gesprochen. Genau wie Techniken und Verkehrsmittel im allgemeinen prägen auch Medien das Verhältnis von Raum und Gesellschaft. „Eine nur geographisch bestimmte Vorstellung“, kommentiert Carl Schmitt im Anschluss an den "Meister der geopolitischen Wissenschaft": Karl Haushofer, sei daher nicht ausreichend, denn die „Kraft der raumüberwindenden Mächte“ sei „zu groß“, um sich mit einer zweipoligen Dialektik von Raum und Gesellschaft zu begnügen (vgl. Schmitt 1991/1941, S. 29). Man müsse daher dreipolig ansetzen: Raum, Technik (Medien), Gesellschaft. Als raumüberwindende Mächte im Weltmaßstab führt Schmitt beispielsweise die Schiffe der ,Seestraßen“, die Flugzeuge der "Luftlinien" oder die Röhren der ,pipe-lines“ an (S. 36). Unter dem Begriff „,communications“ fasst der Publizist Walter Lippmann diese Verbindungswege und Verkehrsmittel zusammen (vgl. Lippmann 1943/44, S. 23, 78). Heute könnte man die Glasfaserkabel des Internets und den Orbit der Satelliten hinzufügen (vgl. Sassen 1997, S. $215 \mathrm{ff}$.). Schmitt nennt das „Radio" als exemplarisches Beispiel einer Technik, die den Raum überwindet, da seine ,elektrischen Wellen“ alle „Staatshoheiten“ und geographischen Sonderlagen ignoriert, um lichtschnell um „den Erdball zu kreisen“ (Schmitt 1991/1941, S. 60). Die Grenzfrage wird hier von der Technik gestellt, denn ein Staat, der über solche Technologien verfügt, hat also "Ausstrahlung“ (S. 63) - und sei er so klein wie Quatar. ${ }^{9}$ Daraus folgt umgekehrt, dass jeder Staat ein „Lebensinteresse im vollen Sinne des Wortes" an seinen Verkehrsmitteln hat (S. 36). Ohne seine nachrichtentechnische und logistische Infrastruktur, konkretisiert Schmitt, könnte etwa das „britische Weltreich“ nicht existieren (S. 36). Ohne eine nachrichtentechnische und logistische Infrastruktur gäbe es keine Globalisierung, keine Weltwirtschaft, keine weltweiten Massenmedien, keine full spectrum dominance ${ }^{10}$ und auch kein information warfare, vom e-commerce, e-government oder e-cinema zu schweigen. Der Herr der ,raumüberwindenden Mächte“ ist daher zugleich der Souverän der Weltgesellschaft. Heute müsste man fragen, wer in command \& control der Satelliten, Pipelines, Schiffahrtslinien, Kabelnetze und Fluglinien der Welt ist und wer auf der anderen Seite diese „Adern“ des Weltverkehrs zu bedrohen vermag. ${ }^{11}$ Die Entscheidungen über die konkrete Route eines Transatlantikkabels oder einer Gas- oder Ölleitung sind von allerhöchstem geopolitischen Rang - wenn man denn überhaupt geopolitisch beobachten mag.

7 Und die deutschen Autobahnen? Auch Adolf Hitler zählte zu den Lesern Ratzels.

8 Ratzel: „Raumverhältnisse und Raumbewältigung“, in: Haushofer (Hrsg) (1941): Erdenmacht und Völkerschicksal, Stuttgart, S. 148.

9 Hier sitzt Al-Jazeera, der wichtige arabische Nachrichtensender.

10 Es geht hier um neue Arten der Kriegsfuhrung mittels neuer Medien. Vgl. dazu vor allem die Abschnitte Full Spectrum Dominance und Information Superiority der Joint Vision 2020 der Joint Chiefs of Staff der US Army.

11 Allein die Fragestellung legt bereits eine Antwort nahe, die auf die „Asymmetrie“ der Konflikte abzielt. Gegen Pipelines kann man Terroristen einsetzten, zur Sicherung der Tausende von Kilometern Pipelines braucht man dagegen reichlich regulăre (disziplinierte) Truppen. 
Die Welt wird klein und kleiner, bis McLuhan sie ein ,global village“ nennen kann. Seit dem Ende des 19. Jahrhunderts wird die These vertreten, die in ihren Medien gleichsam geschrumpfte Welt lasse die Gegensätze der Staaten und Völker aufheben und die Nationen in einer Weltfamilie verschmelzen. Alles, was passiert, konstatiert der britische Geopolitiker Halford J. Mackinder 1904 in seinem berühmten Aufsatz „The Geographical Pivot of History“, „,is re-echoed from the far side of globe" und löse mithin weltweite Konsequenzen aus (1904, S. 27ff.). $\mathrm{Da}$ alles nun mit allem zusammenhänge, würde die Weltgemeinschaft immer enger kooperieren statt Krieg führen, hoffen die Optimisten in kosmopolitischer oder weltrepublikanischer Tradition. Man glaubte, so berichtet Friedrich Naumann (1915) skeptisch, an ,eine friedliche Entpolitisierung der Nationen, Zurückdrängung der Streitmöglichkeiten, Hebung aller durch alle." Wer miteinander Handel und Wandel treibe, bekriege sich nicht. Die Teile fügten sich zum Ganzen. Naumann fährt mit einer für die Geschichte der Medienwissenschaften sehr interessanten Überlegung fort:

„Die Folgen dieses Gedankenganges sind auf Grund der ihn weckenden und begleitenden
Verkehrstechnik ganz ungeheure geworden. Es gibt heute in der Tat eine wirtschaftlich
verbundene Menschheit, die Menschheit der Dampfschiffe, Eisenbahnen, Briefe und Tele-
gramme, eine Menschheit der Nähmaschinen, Getreidesilos, Plantagen und Warenhäuser.
Es gibt den Welthandel und die Arbeitsteilung unter den Völkern" (S. 171).

Wo der Austausch herrscht, herrscht Friede; wenn nun die Medien um den ganzen Globus ausgreifen, steht ein goldenes Zeitalter ewigen Friedens bevor. Auch innerhalb der Nationalstaaten hatte die soziale Arbeitsteilung (Emil Durkheim) nicht zum politischen Zerfall geführt, sondern im Gegenteil zur Gründung intern befriedeter, funktionsdifferenzierter Gemeinschaften. Es lag jetzt für viele nahe anzunehmen, dass sich dies im Weltmaßstab dann wiederhole, wenn auch die Dimensionen der Arbeitsteilung global würden. Der dieser Hoffnung zugrunde liegende Gedanke ist Kantianisch. In der Schrift „Zum ewigen Frieden“ (1795) heißt es: „Es ist der Handelsgeist, der mit dem Kriege nicht zusammen bestehen kann, und der früher oder später sich jedes Volks bemächtigt." Welthandel bedeute Weltfrieden (Kant 1984, S. 33).

Naumann dagegen schreibt im zweiten Jahr des ersten Weltkriegs und kann daher alle Weltbürgertumsideen lapidar kommentieren: „Der Krieg hat bewiesen, daß der Austausch allein noch nicht der Friede ist, denn er allein ist keine verwaltende und regierende, keine den Frieden erzwingende Kraft" (Naumann 1915, S. 171f.). Zwar überwinden die Medien wirklich den Raum, so dass entfernte Mächte miteinander Daten und Waren austauschen können, doch können sie eben auch miteinander Krieg führen; und der erste Weltkrieg, der alle fünf Kontinente intensiv betrifft, wird gerade um die Herrschaft über die raumüberwindenden Mächte geführt, die einen Weltkrieg erst möglich machen. Die wirklichen Mächte der Welt ,streiten innerhalb des Austauschsystems mit ihren Mitteln um den Ertrag und um die Oberleitung des Weltapparates" (ebd., S. 172). Dieser Streit hat bis heute nicht aufgehört. Naumanns Skepsis könnte man daher noch heute all 
denen entgegenhalten, die im Austausch schon den Frieden, im Internet schon die Weltgesellschaft finden. Dass dies durchaus nötig ist, zeigt ein Blick in die aktuelle Thematisierung des Medien-Raum-Macht-Syndroms in der System- und Medientheorie.

\title{
2. WELTWEITE INTERAKTION VERSUS GLOBALE KOMMUNIKATION - MEDIEN DER SYSTEMTHEORIE
}

„Die These, daß die Angelegenheiten aller Menschen irgendwie zusammenhängen, dürfte heute kaum Widerspruch finden“", beginnt Niklas Luhmann 1971 einen Aufsatz mit dem Titel „Weltgesellschaft“ (S. 51). Dreißig Jahre später hat man sich daran gewöhnt, für dieses „Irgendwie“ des Zusammenhanges Internet oder ganz allgemein: Medien zu setzen. Ein „Bereich weltweit orientierter Interaktionen“ (S. 57), so Luhmann, hätte ohne Kommunikationstechnologien überhaupt nicht entstehen können. Die „neuen Kommunikationstechnologien“, heißt es auch in Luhmanns letzter, großer Monographie über Die Gesellschaft der Gesellschaft, „bagatellisieren [...] den Platz“, weil alles auch „,anderswo“" stattfinden kann und „trotzdem nahezu gleichzeitig“. Zu den Folgen der neuen Technologien zählt zumal eine „Bagatellisierung des Standortes“. Wo etwas passiert, ist weniger wichtig als die Frage, wann etwas passiert. Die Medien errichten gleichsam einen „Rahmen der Erreichbarkeit von Wahrnehmung und Kommunikation": Es ist kein Problem, wenn alter sich an einem anderen Ort aufhält als ego, denn man kann ja telephonieren, faxen, mailen, chatten, eine Videokonferenz abhalten, etc. Nur wer aus dieser technisch hergestellten "Gleichzeitigkeit allen Weltgeschehens" herausfällt oder besser: exkludiert wird, nimmt an der Weltgesellschaft nicht teil. ${ }^{12}$ Alle anderen hängen ,irgendwie“ zusammen. Diese neue Möglichkeit eines weltweiten Zusammenhanges, den man spätestens seit der Mitte des 19. Jahrhunderts beobachtet und heute „Netzwerk" nennen würde, umschreibt Luhmann 1971 so:

\begin{abstract}
"Weltweite Interaktion ist möglich, wenn und so weit Partner unter allen Menschen gewählt werden können, sofern dies nach dem Sinn der Interaktion wünschenswert ist, ohne $\mathrm{da} ß$ Gesellschaftsgrenzen dies verhindern. Ein Argentinier mag eine Abessinierin heiraten, wenn er sie liebt; ein Seeländer in Neuseeland Kredit aufnehmen, wenn dies wirtschaftlich rational ist, ein Russe technischen Konstruktionen vertrauen, die in Japan erprobt worden sind; ein französischer Schriftsteller in Ägypten homosexuelle Beziehungen suchen; ein Berliner sich auf den Bahamas bräunen, wenn ihm dies ein Gefühl der Erholung vermittelt" (S. 53).
\end{abstract}

Was Luhmann hier auflistet, ist eine selbstverständlich unvollständige Liste der möglichen Funktions- und Leistungsrollen des Menschen in der modernen Gesellschaft. Man könnte ergänzen: Ein amerikanischer Soldat mag im Irak seinen Feind finden, gegen den er sein Land verteidigen kann, und ein französischer

12 Luhmann 1997, S. 152. Zur Exklusion aus der Weltgesellschaft vgl. Luhmann 1995a. 
Arzt in Guinea einen Kranken, den er behandeln will, oder ein deutscher Demonstrant in Seattle oder Davos ein Publikum für sein Anliegen. Weltweite Interaktion, dies dürfte nun deutlicher werden, impliziert keinesfalls weltweite Verständigung. Der Austausch ist noch nicht der Friede. Der Begriff hat aus gutem Grund keinen moralischen Wert. Die Beispiele machen ebenfalls deutlich, dass sich die den Rollen des Händlers, Soldaten, Romanciers oder Ehemannes entsprechenden Funktionssysteme der Politik, Wirtschaft, Kunst, Wissenschaft oder Liebe ihre Reichweite nicht von Landesgrenzen beschränken lassen; sie wollen sich niemanden entgehen lassen: keinen möglichen Partner, keinen möglichen Kunden, keinen Feind, keinen Leser, kein Patent, keinen Patienten und keinen Profit, egal, wo sich die Klienten dieser Funktionssysteme aufhalten mögen. Im epochalen Gegensatz zu den exklusiven Schichten, Zünften und Ständen der alteuropäischen Gesellschaft sind moderne Funktionssysteme hochgradig inklusiv. Ihr Wirkungsradius ist die Welt. Luhmann hat 1997 erneut betont, in der Weltgesellschaft würden die Grenzen zwischen Staaten „weder von Wahrheiten noch von Krankheiten, weder von Bildung noch vom Fernsehen, weder vom Geld noch von der Liebe respektiert werden", da diese sogenannten ,symbolisch generalisierten Kommunikationsmedien'13 ihrer Funktionssysteme ,unabhängig von Raumgrenzen“" operieren (Luhmann 1997, S. 166). Luhmann selbst lässt hier offen, was Raum ist, daher bleibt auch unklar, was negiert oder bagatellisiert wird. Es scheint mir jedoch, dass der Begriff der Raumgrenze dem Begriff der Lineargrenze entspricht, gegen den die deutsche Geopolitik stets polemisiert hat, um einen biologischen Grenzbegriff zu promovieren, der in der Grenze die semipermeable Peripherie eines Organismus versteht, des Organismus des Volkes, das in seiner Raumnische evoluiert. Die Grenze sei mehr als ein Strich auf einer Landkarte, wie sie „der Gelehrte durch Messung oder die Diplomatie durch einen Vertrag" festsetze, ${ }^{14}$ vielmehr sei sie nach dem Vorbild der Haut eines Lebewesens der periphere Teil eines lebendigen Organismus. Die Grenzen eines Volkes, eines Staates, einer Gesellschaft, so Ratzel, seien ,ihrem Wesen nach veränderlich“, ja lebendig, mit Willen beseelt. Daher sage man: „Die Grenze schiebt sich vor" (Ratzel 1941, S. 33). Die Systemtheorie will nun freilich nicht die Lineargrenze durch ein Lebensraum-Konzept ablösen, sondern löst auch noch den mathematisch-geographischen Raumbegriff auf. Die Medien der Systemtheorie sind mehr als raumüberwindende Mächte, sie schaffen die Notwendigkeit der Rücksicht auf den Raum für die Kommunikation ab.

13 Luhmann 1973, S. 172ff.

14 Ratzel 1941, S. 35. 


\section{KOMMUNIKATION IN DER WELTGESELLSCHAFT}

Weltweite Interaktion: Dies scheint 1971 für Luhmann zunächst einmal zu bedeuten, dass wir leibhaftig von Berlin auf die Bahamas fliegen, um uns zu erholen. Interaktion ist Interaktion unter Anwesenden (vgl. Kieserling 1999). Der Raum spielt dabei weiterhin eine gravierende Rolle, denn Körper müssen von einem Ort zum anderen bewegt werden, um zu interagieren. Dies gilt grundsätzlich für alle res extensa, etwa für Waren und Güter, für Viren und Raketen. Ganz anders verhält es sich mit der Kommunikation. Erst hier könnte man von einer echten „Aufgabe des Raumprinzips“ (Luhmann 1971, S. 61) ausgehen, denn Kommunikation ist allein auf Verkettung angewiesen, also im Grunde auf sich selbst, nicht aber auf die physische Anwesenheit von Menschen. Kommunikation setzt zwar den physischen Raum als Umwelt sozialer Systeme voraus, doch reproduziert sich Kommunikation im gänzlich nichtphysikalischen, körperlosen Medium des Sinns. In Luhmanns Theoriesprache heißt dies Autopoiesis. Bekanntlich ist es die Kommunikation, die kommuniziert, und nicht etwa der Mensch. Anders als die weltweite Partnervermittlung läuft die Kommunikation „menschenfrei" (vgl. nur Luhmann 2002, S. 250). Kommunikation überträgt auch nichts, sie transportiert auch keine Information von einem Lebewesen zum anderen. Alter hat nicht plötzlich ein Substrat, eine Sache, irgend etwas, was ego selbst dann nicht mehr besäße. ${ }^{15}$ Es gibt auch „keinen direkten Zugriff physikalischer, chemischer, biologischer Vorgänge auf Kommunikation" (Luhmann 1997, S. 114). Und den „Komponenten“ der Kommunikation: „Information, Mitteilung und Verstehen“ entspricht nichts, was es in der Welt geben würde. „Weder kann man davon ausgehen“, schreibt Luhmann in der Gesellschaft der Gesellschaft, ,daß es zunächst eine Sachwelt gibt, über die dann noch gesprochen werden kann; noch liegt der Ursprung der Kommunikation in der "subjektiv" sinnstiftenden Handlung des Mitteilens; noch existiert zunächst eine Gesellschaft, die über kulturelle Institutionen vorschreibt, wie etwas als Kommunikation zu verstehen sei." Was man, nach aller negativen Theologie, positiv sagen kann, ist, dass die Kommunikation sich selbst voraussetzt: „Die Komponenten der Kommunikation setzen sich wechselseitig voraus; sie sind zirkulär verknüpft. Sie können daher ihre Eigenschaften nicht mehr als Eigenschaften der Welt ontologisch fixieren" (ebd., S. 72). Hinzu kommt ihre enorme Flüchtigkeit, denn eine Kommunikation ist ein Ereignis, das noch im Moment des Ereignens sogleich wieder zerfällt (vgl. ebd., S. 74). Für die Kommunikation, so schreibt Peter Fuchs in seinem Buch Die Metapher des Systems, komme es daher in gewisser Hinsicht ,nicht darauf an, ob man in Urwäldern, auf Savannen, in Groß Wesenberg, Dinkelsbühl, New York oder Kalkutta lebt oder einstens lebte im Rom der Gladiatoren oder in China vor dem Bau der Großen Mauer oder in Höhlen“. Und weiter heißt es dort: „Kommunikation läuft in der Umwelt von Kannibalen, Terroristen, Benediktinern, Pharaonen so gut ab wie auf Campingplätzen an Ruhr und Lippe" (Fuchs 2001, S. 238). Man möchte gleich widersprechen, aber es geht vorerst um eine Bestandsaufnahme system-

is Vgl. Luhmann 1995b, S. 20; 1997, S. 194. 
und medientheoretischer Positionen, die allesamt einen konstruktivistischen Kommunikationsbegriff gemein haben, aus dem dann die Anschlussthese abgeleitet wird, dass im Zeitalter avancierter Kommunikationsmedien Raum und Körper keine Rolle mehr spielten. Die Positionierung von Körpern (von Kannibalen, Terroristen, Benediktinem, Pharaonen...) im Raum (der Urwälder, Savannen, Dörfer und Städte...) spielt für die Kommunikation keine Rolle, denn Raum und Körper gehören wie der Mensch und sein Bewusstsein zu den „Umweltbedingungen" der Kommunikation (vgl. Luhmann 2002, S. 112), und mit der Umwelt der Kommunikation befasst sich die Systemtheorie der Gesellschaft nicht, weil sie zwar alles Soziale beobachtet, aber alles andere nicht (vgl. Luhmann 1984, 32ff.). Was nicht letztlich in die soziale Elementareinheit der Kommunikation dekomponiert werden kann, gehört nicht zum Objektbereich der Soziologie (ebd., S. 54). Körper und Räume aber sind keine Kommunikationen. ${ }^{16}$

Der Kommunikationsbegriff sieht also vom physischen Raum und von Körpern ab. „Man kann ziemlich rasch sehen [...], dass Kommunikation über eigene Differenzen läuft und nichts mit chemischen oder physikalischen Phänomenen zu tun hat" (Luhmann 2002, S. 113). Richtig. Muss man aber daraus folgern, dass die Verbreitungstechnologien, die allesamt Kommunikation als ein physisches Phänomen behandeln, sich zur Kommunikation selbst indifferent verhalten? Darf der physische Raum, in dem alter und ego kommunizieren, deshalb keine Rolle spielen, weil er selbst keine Kommunikation ist, sondern nur zum Horizont der Kommunikation gehört? Wo ist der Wahrnehmungsbezug geblieben, der bei der Interaktion als ,negationsfeste Prämisse für Kommunikation" 17 ernst genommen wird und die Wahrscheinlichkeiten bestimmter Anschlusskommunikationen, oder genauer: Anschlussinteraktionen, mitbestimmt? Auch das, was man wahrnimmt, ist keine Kommunikation! Und spielt der Standort nicht allein schon deshalb eine Rolle, weil er die Wahl der Medien mitbestimmt und wir von Kanada aus nicht mit dem Mobiltelefon anrufen, weil es dort nicht funktioniert, und daher seit langer Zeit einmal wieder eine Telefonzelle aufsuchen und Kleingeld für ein Ferngespräch sammeln? Wenn Kommunikation aus der dreifachen Differenz von Information, Mitteilung und Verstehen besteht und Information mit Gregory Bateson als Unterschied verstanden wird, der einen Unterschied macht, wird dann nicht jedes Medium der Kommunikation zum Teil einer solchen Differenz, weil es beispielsweise einen Unterschied macht, ob man zum Geburtstag eine Email schreibt, anruft, ein Fax schickt oder einen handschriftlichen Gruß absendet, selbst wenn der Text der Mitteilung stets ,derselbe' wäre? Dies Experiment kann jeder selbst unternehmen. Macht nicht das Medium selbst einen Unterschied, der die Art der Anschlusskommunikation mitbestimmt, weil alter auf egos hochper-

16 Man kann jedoch mit Rücksicht auf Körper oder Räume kommunizieren, so wie man unter Beachtung von Zeit, Sachreferenz oder soziale Differenzen kommunizieren könnte, ohne dass Zeit, Themen oder soziale Positionen selbst schon Operationen der Kommunikation wären. Es handelt sich hier um, ,Sinndimensionen“.

17 Kieserling 1999, S. 129. Interaktion, das sei hier noch einmal expliziert, ist ein Sonderfall von Kommunikation. 
sönlichen, handschriftlichen Glückwunschbrief anders anschließt als auf eine Email vom Maildämonen von birthday.com?

Mit dem Wechsel von der Interaktion unter Anwesenden zur Kommunikation von Abwesenden als Modell für Weltgesellschaft beschreitet die Systemtheorie den Weg einer Bagatellisierung des Raums. Ohne auch nur annähernd klarzustellen, was Raum oder Raumgrenzen überhaupt seien, wird die Bagatellisierung eingeleitet und bis zur Aufhebung des Raums im Nirgendwo weitergeführt. Jedem Ansatz, der nach dem Verhältnis von Geographie, Medientechnologien und Regionalkulturen fragt, wird damit buchstäblich der Grund entzogen. Im „Atopia“18 gibt es keinen Raum für Internetgeographie. Schon Herbert Marshall McLuhan (1992/1964) hat die „neue Welt des globalen Dorfes“ (S. 113) als Folge der „Aufhebung des Raums“ in den elektronischen Medien verstanden: „Die wichtigsten Faktoren des Einflusses von Medien auf bestehende Gesellschaftsformen sind Beschleunigung und Aufteilung. Heute ist die Beschleunigung fast total und macht so dem Raum als Hauptfaktor der sozialen Ordnung ein Ende" (ebd., S. 115). Die „Zentrum-Peripherie-Struktur" der Weltordnung (erste Welt / dritte Welt, Metropolen / Provinz) macht einer mediengestützten „Homogenität“ Platz. „Peripherien hören auf unserem Planeten auf zu existieren“ (ebd., S. 111). Das in der Differenz von Peripherie und Zentrum angelegte Machtgefälle verliert seinen Halt. ${ }^{19}$ Diese Hypothese McLuhans haben deutsche Medien- und Systemtheoretiker übernommen und ausgebaut.

Niklas Luhmann und Rudolf Stichweh haben mit dem Stichwort „Weltgesellschaft“ jener neuen Tatsache Rechnung getragen, dass es zum ersten Mal in der Geschichte nur eine einzige Gesellschaft gibt. In ihr wird mit potentiell globaler Reichweite kommuniziert. Dies war vorher anders: Kommunikationen im präkolumbianischen Maya-Reich, im Australien der Aborigenes oder im alten Tibet hatten nicht die geringsten Konsequenzen in Europa - die Anschlusskommunikationen blieben auf die jeweiligen regionalen Gesellschaften beschränkt. So konnten einst Hochkulturen vergehen, ohne dass dies woanders überhaupt bemerkt wurde, während in der einen Weltgesellschaft ein schwacher Dollar, eine heimtückische Lungenseuche, ein neuer Roman, ein Attentat oder ein neuer Mobilfunkstandard weltweite Konsequenzen bewirkt. Wer es heute noch einmal mit Festungswirtschaft, geschlossenen Grenzen, gesperrten Telekommunikationsleitungen und Einfuhrverboten versuchen will, kann dies nur in der Weltgesellschaft tun - und der Rest der Welt wird sich zum Versuch dieser Abschottung verhalten und feed back in der vermeintlichen Festung geradezu erzwingen. Das historisch Neue an der Weltgesellschaft ist also die Tatsache: „Gesellschaft oder Weltgesellschaft kommt nur noch einmal vor. Es gibt keine anderen Gesellschaften oder Weltgesellschaften neben ihr" (Stichweh 2000, S. 241). Das stimmt. Und ihr Medium ist die Kommunikation, die auf die Anwesenheit der Akteure nicht angewiesen ist. Auch dies klingt evident, man denke nur an internationale Finanzmärkte, an den Erkenntnisgewinn der internationalen science community oder an die globalen

18 So der paradigmatische Titel eines Buches von Helmut Willke 2001.

19 Vgl. dazu Werber 2003. 
Massenmedien. Sie vernetzen „Abwesende“. Und für „wünschenswerte“ Performanz der entsprechenden Systeme reicht Kommunikation vollkommen aus. Man muss nie selbst in den USA gewesen sein, um dort zu publizieren, zu handeln oder zu klagen. Für die verschiedenen Theorien der Weltgesellschaft, wie sie zur Zeit von Helmut Willke, Rudolf Stichweh oder auch Norbert Bolz vertreten werden, spielt daher der Kommunikationsbegriff eine zentrale Rolle. Dies leuchtet ein. Es würde wenig Sinn machen zu formulieren, die Weltgesellschaft bestehe aus der Gemeinschaft jener, die sich gegenseitig besuchen können, um dann face-to-face zu interagieren. „Gesellschaft", so formuliert Willke im Anschluss an Luhmann, sei vielmehr das „System aller füreinander erreichbaren Kommunikationen, das umfassende Sozialsystem aller Kommunikationen. Gesellschaft ist dann zwingend Weltgesellschaft" (Willke 2001, S. 118f.). Aber was macht die Kommunikationen weltweit füreinander erreichbar?

„Die Tatsache eines weltweiten Kommunikationssystems kann nicht bestritten werden", heißt es in Luhmanns postum im Jahre 2000 erschienenen Werk über Die Politik der Gesellschaft fest (S. 220). Statt Interaktion, wie 1971, steht hier Kommunikation. Zwar erinnert Luhmann im selben Buch nochmals daran, „Kommunikation“ sei „an sich keine raumgebundene Operation“ (ebd., S. 263), doch hält er immerhin „Kommunikationstechnologien“ für notwendig, die den Raum des „weltweiten Kommunikationssystems“ überbrücken (ebd., S. 220). Freilich machen „Raumgrenzen", so Luhmann in der Gesellschaft der Gesellschaft, in der Weltgesellschaft „,keinen Sinn“ (Luhmann 1997, S. 809). ${ }^{20}$ Manfred Faßler spricht von einem „cybernetic shift“ zur „Delokalisierung“ (1999, S. 153, 161). Norbert Bolz schließlich bestätigt: Die „elektromagnetischen Wellen haben den Raum so ,erobert', daß er sich zugleich aufgelöst hat. [...] Territorialität ist keine sinnvolle Sinngrenze mehr." 21 Raum scheint hier das Territorium der Nationalstaaten zu meinen, an deren Bagatellisierung und Aufhebung die Theoretiker der Weltgesellschaft gleichfalls keinen Zweifel haben.

Wer sich für den physischen Raum nicht interessiert, kann auch die Medien vernachlässigen, die ihn überwinden. Die Gleichgültigkeit gegenüber den Medien der Weltgesellschaft kann daher nicht überraschen, Luhmann ist überzeugt, dass Medientechniken sich „völlig neutral" zur Kommunikation verhalten (vgl. Luhmann 1997, S. 302). Eine "globale Kommunikationsinfrastruktur" wird vorausgesetzt für den Vollzug von Weltgesellschaft, ${ }^{22}$ doch wird die mediale Verfassung dieser Infrastruktur nicht näher untersucht, weil sie für die Kommunikation keinen Unterschied mache. Sie mache keinen Sinn. ${ }^{23}$ Zwar gilt Kommunikation als das Medium der Gesellschaft, aber inwieweit die Kommunikation in der Weltgesell-

20 Ganz ohne raumüberwindende „Verbreitungsmedien“ geht es nicht (vgl. Luhmann 1997, S. 203), denn die „weltweite Kommunikation" wird in der Weltgesellschaft „telekommunikativ realisiert“ (S. 809). Insbesondere die „neuen Medien“, so Luhmann, „haben die Kommunikationsmöglichkeiten beträchtlich erweitert" (S. 311). Spezifischer wird es jedoch nicht.

21 Bolz 2001, S. 38. Man könnte derartigen Formulierungen die implizite Aussage entnehmen, dass der Raum einmal eine Sinngrenze gewesen sei.

22 Vgl. Stichweh 2000, S. 122. Für Technik insgesamt: Luhmann 1997, S. 532.

23 Vgl. dazu auch Hagen 1999, S. $143 \mathrm{ff}$. 
schaft Telekommunikation oder Verkehr ist und daher auf ganz bestimmte Technologien angewiesen ist, wird nicht gefragt. Die Systemsoziologie blendet die Spezifität von Medientechniken aus: Luhmanns für diesen blinden Fleck exemplarisches Buch über die Realität der Massenmedien (1996) macht keine Unterschiede zwischen Funk und Fernsehen, elektronischer Datenübermittlung und Druck. Luhmann ist die Rücksicht auf „unterschiedliche Kommunikationsmedien“ als Mechanismen der sozialen Evolution, wie wir sie etwa von Harold Adams Innis kennen (vgl. Innis 1997, S. 234), vollkommen fremd. Noch immer scheint es sich so zu verhalten, wie Carl Schmitt vor 70 Jahren festhielt: „scheinbar gibt es nichts Neutraleres als die Technik. Sie dient jedem so, wie der Rundfunk für Nachrichten aller Art und jeden Inhalts zu gebrauchen ist, oder wie die Post ihre Sendungen ohne Rücksicht auf den Inhalt befördert" (Schmitt 1994/1940, S. 146). ${ }^{24}$ Auch das Internet wäre also eine neutrale Technik, die jeder für Nachrichten aller Art und jeden Inhalts gebrauchen mag? Mit Schmitt darf man hier skeptisch sein, denn neutral ist die Technik eben nur scheinbar.

Wir fassen zusammen: Der Begriff der Kommunikation wird in der Systemtheorie jenseits aller technischen, räumlichen oder sozialen Konkretheit konzipiert. Das auf diesem Kommunikationsbegriff basierende Konzept der Weltgesellschaft scheint sich gleichfalls für ihr medientechnisches und geographisches Apriori nicht sonderlich $\mathrm{zu}$ interessieren, denn die Technologien der Verbreitungsmedien verhalten sich zur Kommunikation ja „völlig neutral". Aber wie auch politische Neutralität stets noch für jeden etwas anderes bedeutet hat, stattet auch die Systemsoziologie diese Neutralität der Medien mit einem Programm aus, das um so ungestörter ablaufen kann, weil es ja ein neutrales Programm sein soll, das dem gesellschaftlichen Zugriff entzogen ist. Ich zitiere dieses Programm in der Variante, die Helmut Willke vertritt:

\begin{abstract}
„Die atopische Gesellschaft muss sich in einer Welt ohne Land einrichten. Im Horizont des Atopischen verlieren sich die Stützpunkte erdenschwerer Verlässlichkeit und gravitätischer Traditionen. Sie machen einer konnektivistischen Fluidität Platz, deren Muster und Gestalten kommunikativ konstituierte Figuren bilden und die als Verdichtungen von Kommunikationen sich ebenso schnell auflösen können, wie sie entstanden sind“ (Willke 2001, S. 175).
\end{abstract}

\title{
4. ATOPIA - DIE RAUM-, KÖRPER- UND GEWALTLOSE MEDIENWELTGESELLSCHAFT
}

In dieser Hymne an die ortlose Fluidität skizziert Helmut Willke sein „Atopia“, eine globalisierte Gesellschaft, für die „Ort, Raum und Entfernung [...] zunehmend zu vernachlässigenden Größen" werden (Willke 2001, S. 13). Seine Diagnose klingt vertraut: „Globale Infrastruktursysteme der Telekommunikation und der Verkehrstelematik, global präsente Massenmedien und Transaktionsnetze

24 Damit ist es heute vorbei. Es werden von der Post eigens Technologien beschaff, um die Sendungen zu neutralisieren: gegen Bakterien und Viren. 
bagatellisieren den Platz, von dem aus man kommuniziert, bagatellisieren also Örtlichkeit" (ebd.). Willke folgert nun aus der bereits im Kommunikationsbegriff selbst angelegten Raumlosigkeit der atopischen Gesellschaft das Ende des politischen Hegemonialstrebens und das Ende der Geopolitik. „Wenn Verortung und Ortbarkeit verloren gehen“, so Willke, dann stehe auch die „Überwindung hegemonialer internationaler Regimes" auf der Tagesordnung (ebd., S. 198, 189). „Verortung und Ortbarkeit", Begriffe aus der Geopolitik Karl Haushofers und Carl Schmitts, stehen hier für die militärischen und politischen Voraussetzungen des Freund-Feind-Denkens. Wenn nun aber diesen Voraussetzungen des Politischen die Voraussetzung entzogen wird, weil es keinen Raum mehr gibt, in dem der Feind steht, dann werde zugleich auch, so Willke, den „Plagen wie Fremdenhass, Chauvinismus, Nationalismus, Diktatur und Krieg, welche die Nationalstaaten über die Menschheit gebracht haben“, die Basis entzogen und „obsolet" gemacht (ebd., S. 221). „Regierungsorganisationen, die sich über Landmassen definieren, werden ins Hintertreffen geraten", prophezeit auch Faßler (1999, S. 153). „In den nicht-lokalen Räumen der Medien" (S. 8), so Faßler, verlieren die alten Hierarchien (S. 42) der ,kontinental-geographischen“ Moderne (S. 36) ihre Bedeutung. Zumal der politische Wert des Raums, meint Willke, ,inflationär an Wert" verliere. Infolgedessen schwinde die Bedeutung politischer Macht, die ja letztlich an die ,glaubhafte Androhung physischer Gewalt" gebunden war. Das Netzwerk der globalen Kommunikationssysteme kommuniziere machtfrei, da diese - selbstreferentiell wie die Kommunikation selbst - die ,elementaren Werte und Regeln ihrer Selbststeuerung" selbst festlegen (Willke 2001, S. 230). Mit dem „Ende des Nationalstaates“ (ebd., S. 36, 86, 122) beginne dann das schon zitierte Atopia der „konnektivistischen Fluidität“ (S. 175), in dessen unendlichem Meer alle alten Mächte und Hegemonien endlich vollständig deterritorialisiert untergehen (S. 189). Diese friedliche, fluide und freie Weltgesellschaft entspricht zwar nicht der geopolitischen Realität, aber sie ist sauber aus den systemtheoretischen Grundlagen deduziert. Wir haben es hier mit normativen Implikationen der Theorie zu tun.

Aber nicht nur Willke gelangt derart von der Deskription zur Präskription. Norbert Bolz kommt in seinem neuen Buch Weltkommunikation zu den gleichen Schlüssen (vgl. Bolz 2001, S. 38ff.). Er setzt Niklas Luhmanns raumlose Theorie der Weltgesellschaft gegen Carl Schmitts politisches Raumdenken. Schmitt habe den ,politischen Raum“ vom „Land, dann vom Meer und schließlich von der Luft her gedacht". Staatliche Ordnung werde von ihm territorial verstanden. Das Erkennen von Freund und Feind erfordere „Ortung“. Tatsächlich setzen alle zentralen politischen Kategorien Schmitts einen Raum für Körper und Dinge voraus; die „kommunikative Erreichbarkeit“ der Personen, dies betont Bolz eigens und mit Recht, reiche dafür bei weitem nicht aus. Die ,neuen Kommunikationsverhältnisse", so lautet seine These, haben nun aber diesem Raumdenken jeden Boden entzogen. Der persönliche Standort eines Teilnehmers der Weltkommunikation sei „gleichgültig“ geworden. Die „Weltgesellschaft kann man nicht mehr verorten." Der Territorialstaat erweist sich in dieser Perspektive als Relikt, das nicht etwa Flugzeugen oder Cruise Missiles, sondern allein den grenzenlosen Kommu- 
nikationen der Funktionssysteme nichts mehr entgegenzusetzen vermag. Was Bolz hier als „Bedeutungsschwund des Raums“ bezeichnet, führe aus technischen Gründen und daher unaufhaltsam in eine Weltgesellschaft, in der ,geographische Grenzen, Geschichte (Tradition) und Nationalstaatlichkeit keine Rolle mehr spielen" (ebd., S. 53). Sein Fazit lautet: An Staat und Territorium könne man nur noch „katechontisch“ oder ,anachronistisch festhalten“ (S. 45). Wenn Bolz derart Luhmann gegen Carl Schmitt und alle Geopolitik ausspielt, lässt er das Raumdenken wortwörtlich „alt“ aussehen. Es sei anachronistisch, obsolet, überholt, und nur konservative Phantasten hielten noch daran fest. Doch wird tatsächlich nur ein teleologisches Geschichtsmodell re-etabliert, das historische Phasen kennt, die wie bei einer mehrstufigen Rakete ausgebrannt zurückgelassen werden. ${ }^{25}$ Das Freund-Feind-Denken der Nationalstaaten, des polizeilichen und militärischen Zugriffs auf Körper im Raum, der Kämpfe der Kulturen, der Raumnahmen - die Epoche der Weltkommunikation lässt all dies hinter sich.

Der kommunikationstheoretische Zuschnitt der Systemtheorie legt offenbar ein ganz bestimmtes Verhältnis von Raum, Medien und Macht nahe. Die Raumlosigkeit der Kommunikation generell und im besonderen das Atopia auf Weltniveau nimmt der Macht, verstanden als „Möglichkeit, Räume mit Körpern zu besetzen und [Körper] aus Räumen zu verdrängen“ (Gumbrecht 1999, S. 331), ihr quasi „präsentistisches“ Apriori. Und weil der Medienbegriff semantisch vollkommen neutral gehalten wird, besteht keine Gefahr der Politisierung der Medien der Kommunikation, so dass dem Sprung der atopischen Weltgesellschaft aus dem blutigen geopolitischen Raum der Nationalgeschichte kein Hindernis im Wege steht. Luhmanns unscheinbare und prima vista völlig evidente Formel von der „Bagatellisierung des Standortes“ (Luhmann 1997, S. 152) wird so innerhalb der Medien- und Systemtheorie radikalisiert zur Vision einer total deterritorialisierten Weltgesellschaft. Die Geopolitik hat ihren Boden verloren, es gibt nur noch Meer, keine Ufer. Aller ,politischen Geographie“, wie sie in Deutschland von Friedrich Ratzel bis Karl Haushofer betrieben worden ist (vgl. Ratzel 1897), und ihrer „kritischen“ Fortsetzung ${ }^{26}$ fehlen nun die Grundlagen, denn die Einfügung des Menschen in seine Umwelt wird nicht länger von Lage und Landschaft mitgeprägt, ${ }^{27}$ sondern allein von der Struktur virtueller Netze. Die digitale Welt funktioniere daher ,,vollkommen unabhängig von geopolitischen Grenzen“ (Negroponte 1995, S. 276). Im virtuellen Raum, so schreibt Faßler, ,herrscht selbst bei herkömmlichen Beziehungen Telepräsenz, da der über das elektronische Netz, die Mailbox usw. aufgerufene Informationsraum unabhängig von Territorialität, Biosphäre, Geographie usw. rechnend existiert" (Faßler 1999, S. 175). Hegel hatte den Menschen einst den „Sohn seines Bodens“ genannt und damit gemeint, dass ein Volk von der geographischen Nische geprägt wird, in der es evoluiert

25 Vgl. als siebenstufige Rakete die Phaseneinteilung bei Faßler 1999, S. 36.

26 Vgl. etwa Geopolitik. Kritische Geographie 14, Wien 2001, oder Reuber/Wolkersdorfer 2001 .

27 „Die wichtigsten davon sind Größe, Lage und Grenzen, dann die Art und Form des Bodens samt seiner Bewachsung und seinen Gewässem und endlich sein Verhältnis zu anderen Teilen der Erdoberfläche“ (Ratzel 1941, S. 113). 
(vgl. Hegel 1986, S. 106). Ratzel hat diesen Gedanken aufgegriffen, wenn er schreibt: „In die Geschichte des Volkes, dem es gelungen ist, Jahrhunderte auf gleichem Boden seinen Staat zusammenzuhalten, prägt diese unveränderliche Grundlage sich so tief ein, daß es nicht mehr möglich ist, dieses Volk ohne seinen Boden zu denken" (Ratzel 1941, S. 114). Diesen Boden hat die Weltgesellschaft nun aber verloren. Ihr Medium ist nicht der Raum der Geopolitik, sondern der Cyberspace - meinen die System- und Medientheorie.

\section{MEDIEN UND GEOGRAPHIE IM ZEITALTER DER GEOPOLITIK}

Warum eigentlich? Es könnte zum historischen Erbe der deutschen Soziologie und Medientheorie gehören, dass sie die Frage nach dem Raum nicht stellen mag - nach dem Untergang des Nationalsozialismus wurde die geopolitisch orientierte Forschung und ihre Sicht auf Medien als ,raumüberwindende Mächte' pauschal diskreditiert und ihre Begriffe und Forschungsinteressen tabuisiert. Die Geisteswissenschaften haben infolgedessen die daran anschließenden Fragen nach der Macht und der Technik nur noch ignorieren können. „Was hat die physische Landkarte mit politischer Realität zu tun?“, fragt im Jahre 2001 ein Professor für die Didaktik der Geographie und antwortet: „Nichts“, wenn man sich an „Wissenschaftler von heute" halte (vgl. Schultz 2001, S. 29). Gottseidank, denn das 19. Jahrhundert hegte aufgrund der Verkettung von Politik und Geographie gefährliche „Großraumvisionen“ eines Deutschen Reichs zwischen „Nordsee und Schwarzem Meer" (S. 32). Der zweite deutsche Weltkrieg wurde geführt, um diesen Großraum gegen allen Widerstand zu realisieren. Die Vermeidung allen geopolitischen Denkens schien umgekehrt auch die Wiederkehr einer deutschen Großmachtpolitik auszuschließen. Die Semantik der Globalisierung und der World Wide Web-Gesellschaft, der Weltgesellschaft und des Cyberspace verdankt ihre Popularität hierzulande möglicherweise der attraktiven Illusion, den als verhängnisvoll geltenden Raum politischer Souveränität (Mittellage, „Volk ohne Raum“, Bedrohung aus dem Osten, Großraumordnung) endgültig verlassen zu können. Norbert Bolz' Liste aktueller Selbstbeschreibungsformeln: „One World, Internet, Netzwerk, Ökologie, [...] Globalisierung, Multikulturalität“" (Bolz 1997, S. 120) indiziert die Bodenlosigkeit der neuen Semantik. Bolz fügt denn auch gleich hinzu, die „Idee des Postnationalen“ funktioniere als „Ausflucht“, Europa entlaste „von der Undenkbarkeit Deutschlands“ (ebd., S. 124). Auf allen Ebenen: politisch, kulturell, ökonomisch, wissenschaftlich, militärisch tritt die Semantik der Neuen Medien die Flucht aus dem Raum an. Die Internetgesellschaft ist dann selbstverständlich ortlos wie friedlich, multikulturell wie demokratisch. Während sich in den USA hinter den gleichen Formeln das Ziel einer Eroberung des Cyberspace, einer neuen Raumnahme, eines neuen Mittels globaler Hegemonie nicht einmal verbirgt, ${ }^{28}$ suggerieren sie hierzulande die Vorstellung einer gewaltfreien, unhierarchischen, atopischen Weltgesellschaft, die sich medientechnisch auf das 
Internet stützt und „politisch" auf die Netzwerke internationaler Institutionen und NGOs. Ubiquitäre Komposit-Begriffe wie WWW- oder Internet- oder CyberSociety, Wissens-, Info- oder Netz-Gesellschaft lassen den Wunsch erkennen, dass der Macht- und Nationalstaat der Vergangenheit angehören solle. Diese Hoffnungen werden von der Annahme befeuert, aus der technischen Verfassung des Internets erwachse eine entsprechende Verfasstheit der Gesellschaft: lateral, demokratisch, netzwerkartig, inklusiv, gewaltfrei, konsensuell..., ganz als ob die Weltgesellschaft nach dem Vorbild eines Internet-Forums eingerichtet würde. Die Erwartung, aus den neuen technischen Kommunikationsverhältnissen gehe zwangsläufig eine neue soziale Ordnung hervor, ist typisch für die deutsche $\mathrm{Me}$ dientheorie (vgl. Werber 1993). Heute soll das Internet ein Atopia gebären, das im Zeitalter der deutschen Weltkriege nie eine Chance hatte. Diese Semantik propagiert die restlose Integration in die Weltgesellschaft, „um Deutschland den Laufpaß zu geben" (Böckelmann 1999, S. 232). Das Netz, in dem man hierzulande ein Modell einer Weltrepublik zu finden meint, sieht man dagegen in den USA als Herausforderung, sich auch ,durch die Beherrschung der weltweiten Kommunikationssysteme" einen "massiven, aber nicht greifbaren Einfluss" zu verschaffen (vgl. Werber 2000). „Globale Kommunikation“, schreibt Samuel Huntington, ,ist eine der wichtigsten zeitgenössischen Manifestationen westlicher Macht", ja, sie zähle zu den Kernelementen einer „Hegemonie des Westens“ (Huntington 1998, S. 80). Cyber-Deutschland dagegen mache sich, so Böckelmann, zu einem „Terminal ohne Hinterland" (Böckelmann 1999, S. 233).

Unsere Vermutung, die deutsche Medien- und Systemtheorie fliehe aus dem machtpolitisch diskreditierten „Lebensraum“ wird plausibler, wenn man sieht, wie in dieser Semantik das Atopia der Weltgesellschaft der geopolitisch geprägten Epoche des deutschen Sonderweges entgegengestellt wird. „Noch das 19. Jahrhundert", schreibt Stichweh,

„scheint den Prozeß territorialer Zentralisierung voranzutreiben und mit den Vereinigten Staaten und Rußland wurden kontinentgroße Einzelstaaten weltpolitisch dominant, eine Tendenz, die [...] als der Hintergrund des deutschen Expansionismus im 20. Jahrhundert gedeutet werden kann" (Stichweh 2000, S. 23ff.).

Mit diesen Versuchen, macht- und geopolitische Großraumordnungen zu bilden, sei es aber in der Weltgesellschaft vorbei, weil die weltweite Strukturbildung politischer Kommunikation zu einer „Egalisierung nationaler Souveränität" führe. Wie die modernen Verfassungen des Nationalstaats allen Bürgern gleiche Rechte und Pflichten unabhängig von Macht, Rang, Einkommen und Stand einrichteten, würden ,die Nationalstaaten als konstitutive Bürger“ behandelt, also als Gleiche unter Gleichen. In der Weltgesellschaft gibt es für Stichweh also ein einziges System politischer Kommunikation mit globaler Dimension, dessen Spielregeln für große und kleine Staaten genauso gelten wie in den Einzelstaaten für große und kleine Parteien. „Erstmals“, so wird betont, „unterscheiden sich die Überlebenswahrscheinlichkeiten für große und kleine Staaten nicht wesentlich, sind kleine Staaten nicht mehr auf geographische Sonderlagen und hegemoniale Un- 
terordnung angewiesen" (ebd., S. 25). Dieses Ende der Hegemonial- und Machtpolitik großer und kleiner Staaten ist aber wiederum eher eine normative Implikation der Systemsoziologie als eine Beschreibung der politischen Realität der Weltgesellschaft. Stichweh spricht von einer „relativen globalen Homogenisierung“ im Medium der weltweiten „Vernetzung kommunikativer Ereignisse“. Der verwendete Netzwerkbegriff konnotiert ein Mit- und Nebeneinander gleichwertiger Knoten, deren Koordinaten im Raum ebenso unwichtig sind wie die „bagatellisierten" Standorte der telekommunizierenden User. Die Weltgesellschaft, so wird behauptet, dämpfe die ,nationalkulturellen Eigenheiten“ und „Idiosynkrasien“ ab und läute so das Ende der Hegemonien und den Anfang einer „,egalitären“ Weltordnung ein. Und jene „Staaten“, die noch einen expansiven, „,kulturellmissionarischen Zug aufweisen, würden im System der Weltgesellschaft politisch disprivilegiert" (ebd., S. 54).

All dies stützt die systemtheoretische Globalthese, dass die Epoche der Weltgesellschaft das Zeitalter der Hegemonien abgelöst habe oder doch bald ablösen werde oder ablösen müsse. Dass diese Erwartungen eine bestimmte Variante der Interneteuphorie nur auf Weltmaßstab heraufrechnet, scheint mir ganz offensichtlich zu sein, so sehr ähneln sich die Beschreibungen der Cyber-Moderne und der Weltgesellschaft, so sehr entfliehen sie gleichermaßen Räumen, Körpern und Macht und so sehr setzten sie laterale Netzwerke gegen Hierarchien und Hegemonien. Die Entwürfe der Cyber-Moderne und der Weltgesellschaft teilen sich auch das Pathos, das Ende zu verkünden. Das Ende des Raums, das Ende der Nationalstaaten, das Ende der Hegemonien oder das Ende der Gewalt. „Die protektiven Grenzanlagen, wenn sie denn angestrebt werden, schützen nur noch Vergangenes, sind terroristische Folklore verkommener Systeme, wie im Irak, im Iran, in Serbien, Kroatien, in Indonesien oder..." (Faßler 1999, S. 192). Die „globalen Infographien" übernehmen, liest man bei Faßler (ebd., S. 193), und man müsste nun nur noch auf das Vergehen des Vergangenen warten. Das Warten darauf könnte aber unendlich lange dauern. Die Zwischenzeit wird auf eine geopolitisch informierte Soziologie nicht verzichten können, und alle Medientheorie wird sich auf Internetgeographien einlassen müssen, wenn sie nicht nur an Utopien der Internetgesellschaft weiterschreiben will.

\section{LITERATUR}

Böckelmann, Frank (1999): Deutsche Einfalt, München.

Bolz, Norbert (1997): Die Sinngeselischaft, Düsseldorf.

Bolz, Norbert (2001): Weltkommunikation, München.

Budke, Alexandra, Kanwischer, Detlef und Andreas Pott (2002): Internetgeographien. Call for Papers.

Chomsky, Noam (2002): 9-11, New York, S. 112.

Faßler, Manfred (1999): Cyber-Moderne: Medienevolution, globale Netzwerke und die Künste der Kommunikation, Wien u.a.

Fuchs, Peter (2001): Die Metapher des Systems, Weilerswirst. 
Gumbrecht, Hans Ulrich (1999): „Was sich nicht wegkommunizieren läßt“, in: Maresch, Rudolf und Niels Werber (Hrsg.): Kommunikation. Medien. Macht, Frankfurt a.M., S. 329-341.

Günther, Gotthard (2000): Die Amerikanische Apokalypse, München.

Hagen, Wolfgang (1999): „Zur medialen Genealogie der Elektrizität“, in: Maresch, Rudolf und Niels Werber (Hrsg.): Kommunikation. Medien. Macht, Frankfurt a.M., S. 133-173.

Haushofer, Karl (Hrsg.) (1934): Raumüberwindende Mächte, Leipzig/Berlin.

Hegel, Georg Wilhelm Friedrich (1986): Vorlesungen über die Philosophie der Geschichte. Werke Bd. 12, Frankfurt a.M.

Huntington, Samuel P. (1996): Kampf der Kulturen. Die Neugestaltung der Weltpolitik im 21. Jahrhundert, München/Wien.

Innis, Harold Adams (1997): Kreuzwege der Kommunikation, hrsg. v. Karlheinz Barck, Wien/New York.

Kant, Immanuel (1984): Zum ewigen Frieden, Stuttgart.

Kieserling, André (1999): Kommunikation unter Anwesenden, Frankfurt a.M.

Kritische Geographie (Hrsg.) (2001): Geopolitik. Zur Ideologiekritik politischer Raumkonzepte, Wien.

Lippmann, Walter (1942/44): U.S. Foreign Policy and U.S. War Aims, USA.

Luhmann, Niklas (1991): „Die Weltgesellschaft“ (1971), in: ders.: Soziologische Aufklärung. Bd. 2, Opladen, S. 51-71.

Luhmann, Niklas (1973): „Einführende Bemerkungen zu einer Theorie symbolisch generalisierter Kommunikationsmedien“", in: ders.: Soziologische Aufklärung, Bd. 2, Opladen, S.172-192.

Luhmann, Niklas (1984): Soziale Systeme. Grundriss einer allgemeinen Theorie, Frankfurt a.M.

Luhmann, Niklas (1995a): „Jenseits von Barbarei“, in: ders.: Gesellschaftsstruktur und Semantik. Bd. 4, Frankfurt a.M., S. 138-150.

Luhmann, Niklas (1995b): Die Kunst der Gesellschaft, Frankfurt a.M.

Luhmann, Niklas (1996): Die Realität der Massenmedien, Opladen.

Luhmann, Niklas (1997): Die Gesellschaft der Gesellschaft, Frankfurt a.M.

Luhmann, Niklas (2000): Die Politik der Gesellschaft, hrsg. von André Kieserling, Frankfurt a.M.

Luhmann, Niklas (2002): Einführung in die Systemtheorie, Heidelberg.

Mackinder, Halford J. (1998): „The Geographical Pivot of History" (1904), in: Ó Tuathail, Gearóid, Dalby, Simon und Paul Routledge (Hrsg.): The Geopolitics Reader, London, S. 27-31.

McLuhan, Herbert Marshall (1992): Die magischen Kanäle. Understanding Media (1964), Düsseldorf/Wien u.a.

Naumann, Friedrich (1915): Mitteleuropa, Berlin.

Negroponte, Nicholas (1995): Total Digital, München.

Ratzel, Friedrich (1897): Politische Geographie.

Ratzel, Friedrich (1941): Erdenmacht und Völkerschicksal, hrsg. von Karl Haushofer, Stuttgart.

Reuber, Paul und Günter Wolkersdorfer (Hrsg.) (2001): Politische Geographie: Handlungsorientierte Ansätze und Critical Geopolitics, Heidelberg.

Sassen, Saskia (1997): „Cyber-Segmentienungen“, in: Münker, Stefan und Alexander Roesler (Hrsg.): Mythos Internet, Frankfurt a.M., S. 215-235.

Schmitt, Carl (1994): „Das Zeitalter der Neutralisierungen und Entpolitisierungen“, in: Positionen und Begriffe, Berlin (1940), S. 138-150. 
Schmitt, Carl (1991): Völkerrechtliche Großraumordnung mit Interventionsverbot für raumfremde Mächte (1941), Berlin.

Schultz, Hans-Dietrich (2001): „Geopolitik avant la lettre“, in: Geopolitik. Kritische Geographie 14, Wien, S. 29-50.

Stichweh, Rudolf (2000): Weltgesellschaft, Frankfurt a.M.

Walsh, Edmund A. (1946): Wahre anstatt falsche Geopolitik für Deutschland, Frankfurt a.M.

Werber, Niels (1993): „Neue Medien, alte Hoffnungen“, in: Merkur, Heft 534/535, September/Oktober, S. 887-893.

Werber, Niels (1997): „Die Form der Telematik. Zur Semantik der globalisierten Gesellschaft“, in: Merkur, Heft 9/10, Stuttgart, S. 890-901.

Werber, Niels (2000): „Mediale Großraumordnung. Das alte geopolitische Denken ist in die USA emigriert", in: Merkur, Heft 9/10 (617/618), S. 1031-1037.

Werber, Niels (2003): „Centre as a Form. On the Differentiation of Centre and Periphery in Current Semantics with a Regard to Art, Urbanism, Globalisation, and Geopolitics", in: CAC und Tobias Berger (Hrsg.): The Centre of Attraction. Reader, Vilnius.

Willke, Helmut (2001): Atopia. Studien zur atopischen Gesellschaft, Frankfurt a.M. 



\title{
METHODEN UND ANWENDUNGSGEBIETE DER INTERNETKARTOGRAPHIE
}

\author{
Inga Heinze
}

\section{WHERE ON EARTH IS THE INTERNET?}

In den Anfangsjahren lag der Reiz des Mediums Internet u.a. darin, dass sich der User von der Benutzung des Internets den Eintritt in eine völlig neue Welt versprach, die, losgelöst vom physischen Raum, eine autonome Existenz im Cyberspace zu ermöglichen schien. Dies waren zumindest die Vorstellungen und Hoffnungen einiger Internet-Pioniere. Mittlerweile ist in dieser Hinsicht weitgehend Ernüchterung eingekehrt und wohl kaum jemand wird heute noch leugnen können, dass das Internet mit zunehmender Verbreitung und der damit einhergehenden Kommerzialisierung immer stärker die Strukturen der nicht-virtuellen Welt nachbildet. Die Internetkartographie geht genau diesem Phänomen auf den Grund: Mittels kartographischer Darstellungen wird versucht, die Verbindung von Internet und physischem Raum sichtbar zu machen.

Bis Ende der 1990er Jahre beschränkten sich die Darstellungen auf die Erstellung von Netzwerkplänen der an das Internet angeschlossenen Computer und Netzwerke. Seit einigen Jahren ist zu beobachten, dass derartige Darstellungen in zunehmendem Maße auch für die Beantwortung von nicht-technischen Fragestellungen herangezogen werden. Where on Earth is the Internet? - so überschrieben beispielsweise Martin Dodge und Narushige Shiode eine Studie aus dem Jahr 1998, in der der Versuch unternommen wurde, die physisch-räumliche Verbreitung des Internets in Großbritannien durch die Ermittlung der Anschriften von IPAdressen-Inhabern zu erfassen.

Der vorliegende Beitrag grenzt zunächst den Begriff Internetkartographie gegenüber anderen Begriffen ab. Im Anschluss daran werden unterschiedliche Typen der Internetkartographie vorgestellt. Hierbei werden auch die technischen Grundlagen und die gebräuchlichen Methoden kartographischer Darstellungen des Internets aufgezeigt. Darauf aufbauend werden die Anwendungsgebiete und der Nutzen der Internetkartographie diskutiert, um abschließend einen Ausblick auf die Verknüpfung von Internetkartographie und Geographie zu geben.

\section{INTERNETKARTOGRAPHIE - EINE BEGRIFFLICHE ANNÄHERUNG}

Da bisherige Arbeiten zur Internetkartographie überwiegend in englischer Sprache verfasst sind, muss die Terminologie für den deutschen Sprachraum geklärt 
werden. Der Begriff Internetkartographie taucht im Deutschen bisher kaum auf; wenn er verwendet wird, dann als Synonym für die Bereitstellung kartographischen Materials im Internet oder für die kartographische Darstellung des Internets selbst. In der englischen Fachsprache hat sich diesbezüglich eine Differenzierung herausgebildet. So bezeichnen die englischen Begriffe Internet cartography und Web cartography die Veröffentlichung von kartographischem Material oder von Informationen zum Thema Kartographie auf einer Webseite. Der englische Begriff Internet mapping wird dagegen verwendet, um die Kartierung des Internets zu beschreiben.

In diesem Aufsatz wird der Begriff Internetkartographie im Sinne des englischen Begriffs Internet mapping verwendet. Unter Internetkartographie wird also die Kartierung von technischen Komponenten, wie z.B. der Hardware, der Datenströme oder der Netztopologien verstanden. Da die Begriffe Internet und World Wide Web im alläglichen Sprachgebrauch häufig synonym benutzt werden, taucht in jüngster Zeit auch der Begriff Webkartographie vermehrt auf. Der Begriff Webkartographie bezeichnet allerdings die Kartierung von inhaltsbezogenen Aspekten des World Wide Web, wie zum Beispiel vom Navigationsverhalten der Anwender, von Strukturen der Multi-User Environments (vgl. dazu die Abbildungen im Beitrag von Stegbauer in diesem Buch) oder wie bei der Erstellung sogenannter Sitemaps. Im Gegensatz zur Webkartographie wird in der Internetkartographie ein Bezug zwischen dem Untersuchungsobjekt und dem physischen Raum hergestellt. Auf diesen Bezug beschränkt sich mein Beitrag.

\section{TYPEN DER INTERNETKARTOGRAPHIE}

Je nachdem welche technische Komponente oder welches Phänomen des Internets untersucht wird, lassen sich unterschiedliche Typen der Internetkartographie unterscheiden. Im folgenden werden mit der thematischen Unterscheidung von Hardware, Datenaufkommen, IP-Adressen und Zugangsmöglichkeiten vier typische Darstellungsformen näher erläutert.

\subsection{Darstellungen der physischen Internet-Infrastruktur}

Die physische Infrastruktur des Internets umfasst alle Hardware-Komponenten, die zur Datenübertragung erforderlich sind - beispielsweise Datenleitungen, Router, Satelliten oder Server. In den 60er Jahren des 20. Jahrhunderts, als das Internet noch in den Kinderschuhen steckte, gab es erst wenige Bemühungen, diese Komponenten des Internets in ihrem physisch-räumlichen Bezugsrahmen darzustellen. Das Interesse konzentrierte sich zu jener Zeit überwiegend auf die Weiterentwicklung der technischen Grundlagen. Jene Wissenschaftler, die die technischen Voraussetzungen für die heutige Verbreitung des Mediums schufen, können trotzdem als die Urväter der Internetkartographie angesehen werden. 


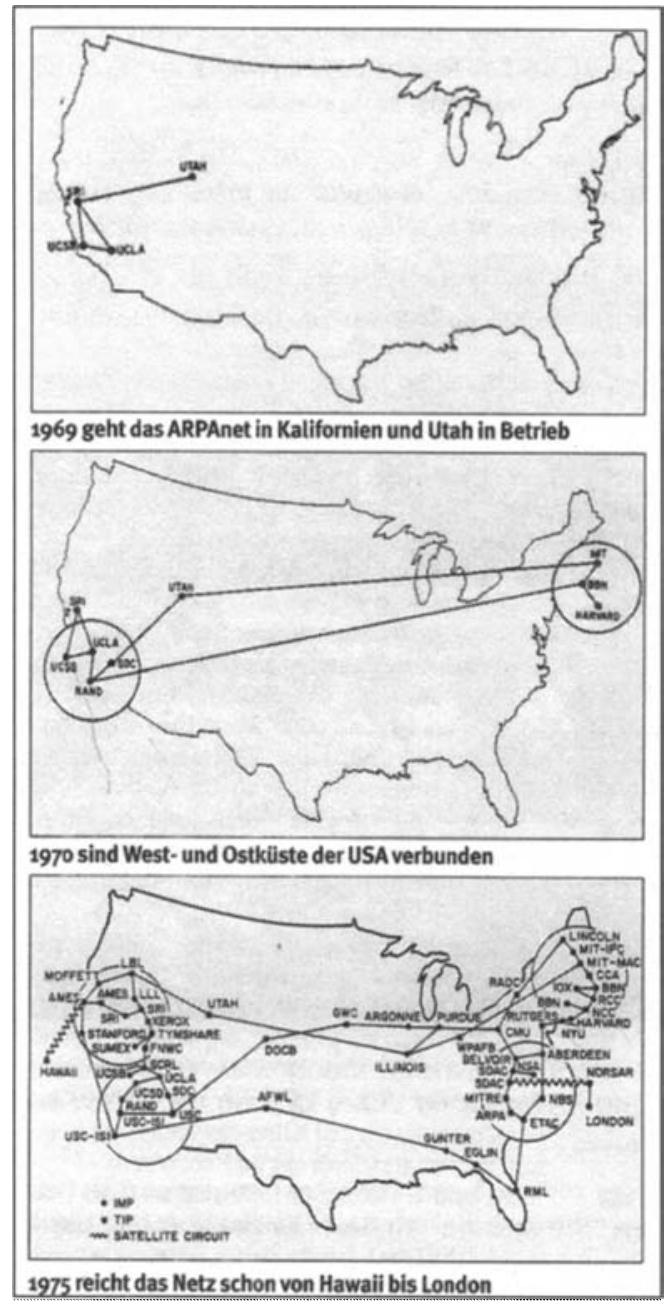

Abbildung 1: Entwicklung des ARPANET (Quelle: Rademacher 2001, S. 73).

Sie kombinierten bereits in den ersten Skizzen technische Netz- und Schaltpläne mit topographischen Informationen, um die räumlichen Dimensionen des Internets zu betonen. Damit schufen sie eine neue Darstellungsform an der Schnittstelle zwischen Geographie und Technik. Die bereits schon legendären Skizzen der ersten Knotenpunkte des ARPANET zeigen, wie problemlos sich diese beiden Bereiche zusammenfügen lassen. Bis in die 70er Jahre war es möglich, in dieser Darstellungsform jeden Host des Internets einzeln abzubilden (vgl. Abb. 1).

Die schnelle Verbreitung der Technologie führte jedoch zu einem rasanten Anwachsen der Zahl beteiligter Rechner, so dass sich seit Ende der 70er Jahre 
neben dem ARPANET weitere Netze als eigenständige Organisationseinheiten entwickelten (z.B. SATNET: Satellitenübertragung, MLNET: Netzwerk des Verteidigungsministeriums, NSFNET: Netzwerk der National Science Foundation). Von diesem Zeitpunkt an war es fast unmöglich, mit der bisherigen Methode übersichtliche Darstellungen des Internets zu erhalten, allenfalls war dies noch bei der Betrachtung einzelner Netze oder Netzabschnitte möglich. Man ging deswegen dazu über, das Internet seiner Natur nach als ein Netz von Netzen darzustellen und nur noch die Netze selbst sowie die Verbindungen zwischen den Netzen aufzuzeichnen:

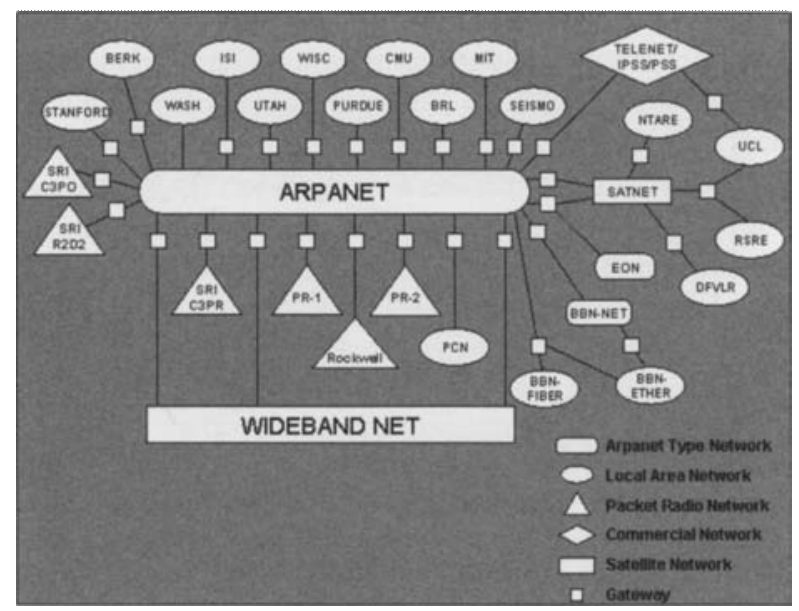

Abbildung 2: Konzeptionelle Darstellung des Internets 1983 (Computer History Museum 2003).

In den 20 Jahren, die seit der Erstellung dieser Graphik aus dem Jahr 1983 vergangen sind, ist das Intemet noch einmal um ein Vielfaches angewachsen, weswegen eine weitere Anpassung der Darstellungsform stattgefunden hat. Um nach wie vor aussagekräftige Karten erstellen zu können, tendiert man dazu, das Internet nur noch ausschnittsweise zu betrachten oder die dargestellten Faktoren sehr stark zu abstrahieren. Eine Methode besteht darin, sich auf die Kartierung von regionalen Ausschnitten zu beschränken. Die zweite Methode abstrahiert von den technischen Komponenten. So findet man z.B. häufig topographische Darstellungen der Backbone-Infrastruktur von Internetserviceprovidern, die nur das Rückgrat, d.h. die besonders gut ausgebauten Hauptdatenleitungen, zeigen (vgl. dazu auch Langhagen-Rohrbach in diesem Band, Abb. 1). Derartige Darstellungen enthalten in der Hauptsache Informationen über Knotenpunkte und die wichtigsten Verbindungen innerhalb des Netzwerks, wie Abbildung 3 am Beispiel des europäischen Teils des Sprint Backbones zeigt. Aus dieser Art der Darstellung lassen sich die differenzierten Funktionen der Knotenpunkte und die unterschiedlichen Verbindungsmedien ablesen. Beispielsweise werden Daten, die 
aus Übersee kommen, zunächst in sogenannten Landing Stations außerhalb der eigentlichen Zentren des Netzes gebündelt und von dort aus an die Zentralen weitergeleitet. $^{1}$

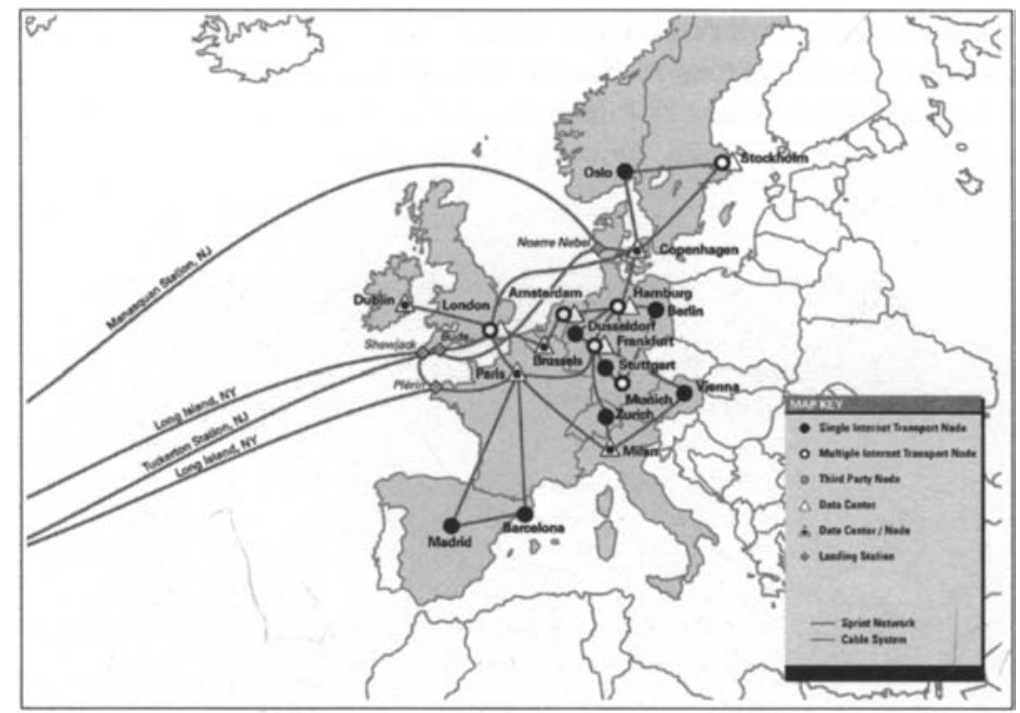

Abbildung 3: Europäischer Teil des Sprint Backbones (Sprint 2002).

\subsection{Auswertung des Internettraffics}

Als zweiter Typus der Internetkartographie kann die Darstellung des Datenaufkommens im Internet, des sogenannten Internettraffics, betrachtet werden. Dieser Bereich umfasst (a) die Analyse des quantitativen Traffic-Aufkommens und (b) die qualitative Auswertung der Paket-Routen. Für beide Arten der Analyse des Datenverkehrs sind zahlreiche Software-Produkte, sowohl kommerzielle als auch frei erhältliche, verfügbar. Aus kartographischer Sicht liegt die große Herausforderung dieser Art von Darstellungen darin, die meist sehr großen Datenmengen so zu abstrahieren, dass aussagekräftige Karten entstehen. Der Aufwand, derartige Karten zu erstellen, reicht von einfachen Programmen, mit denen der Inter-

Man findet Karten über die physische Infrastruktur des Internets am einfachsten über eine entsprechende Anfrage in einer Suchmaschine. Allein die Suche nach dem Begriff "Backbone map" liefert Dutzende dieser Abbildungen. Sehr hilfreich ist die Homepage von Martin Dodge (http://www.cybergeography.org). Diese Seite ist eine der besten Quellen zum Thema Cybergeography. Ebenfalls empfehlenswert ist Russ Haynal's ISP Page (http://navigators.com/-isp.html). Die Cooperative Association for Internet Data Analysis (CAIDA http://www.caida.org) hat sich die Überwachung der Entwicklung der InternetInfrastruktur zum Ziel gesetzt. Auf der Homepage stehen etliche Visualisierungstools zur Verfügung. 
nettraffic am heimischen Computer kartiert werden kann, bis hin zu aufwendigen globalen Trafficanalysen, bei denen zumeist Daten von globalen Netzbetreibern gebündelt verarbeitet werden.

a) Vor allem die quantitative Analyse des Internettraffics ist nur mit umfangreichen Mitteln zu bewerkstelligen. Bisher werden derartige Analysen überwiegend von kommerziellen Anbietern durchgeführt, wobei zwei US-amerikanische Firmen diesen Markt dominieren: Matrix NetSystems (http://www.mids. org) und Tele Geography (http://www.telegeography.com).

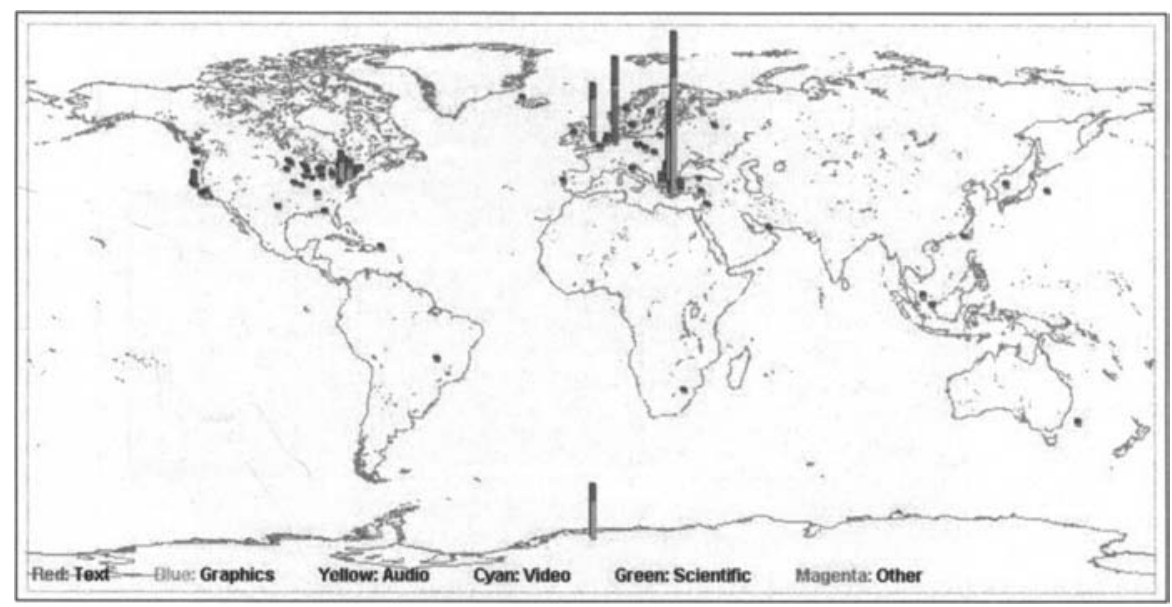

Abbildung 4: Webserveranfragen nach Ursprungsland und Inhalt, dargestellt mit Hilfe des Palantir-Tools (Papadakakis u.a. 1998).

Das Projekt Palantir (http://www.ics.forth.gr/carv/demos/palantir.html) des griechischen Forschungszentrums FORTH (Foundation for Research and Technology - Hellas) kommt mit einem wesentlich geringeren Aufwand der Datenerhebung aus, betrachtet dabei aber auch nur den Internettraffic, der an einen einzelnen Webserver in Griechenland gerichtet wird. Den Inhalt der Analyse bildet dann die Lokalisierung und Kartierung des Ursprungs der Webserveranfragen. Derartige Karten können zum Beispiel bei Fragen der Netzoptimierung herangezogen werden, um die räumliche Verteilung von Webservern zu evaluieren (vgl. Abb. 4)

b) Wesentlich zahlreicher sind die Anwendungen der qualitativen Trafficanalysen, mit deren Hilfe die Wege einzelner Datenpakete durch das Internet verfolgt werden können. Dies geschieht mit sogenannten Traceroute-Programmen. Als einfachstes Mittel beinhaltet nahezu jedes Betriebssystem das Programm „Traceroute“, mit dem die Stationen der Datenübertragung ermittelt und textbasiert ausgegeben werden können. Der Traceroute-Befehl ermöglicht es, die IP-Adressen der Stationen der Datenübertragung zu ermitteln. Programme mit kartographischer Ausgabe ermitteln zusätzlich über eine „Whois“-Abfrage den 
zur IP-Adresse gehörigen Standort und tragen ihn in eine Karte ein. Somit entsteht ein topographisches Abbild der Route, über die ein Datenpaket vom lokalen Rechner durch das Internet geschickt wird. ${ }^{2}$

Ein anderes Darstellungsprinzip ist die Bündelung mehrerer Paket-Routen. Dieses Prinzip liegt der Darstellung von Cox et al. (vgl. Abb. 5) zu Grunde. Sie vermittelt daher einen Eindruck vom Gesamtaufkommen des weltweiten Datenverkehrs.

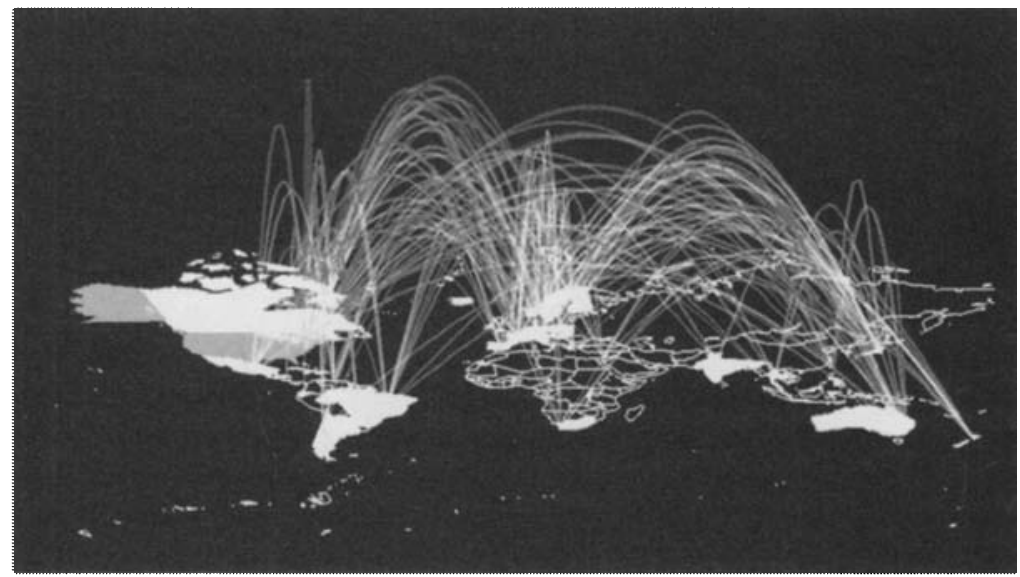

Abbildung 5: Darstellung des weltweiten Internetdatenaufkommens (Cox u.al. 1996).

\subsection{IP-Adressen und Domain-Namen-Kartierung}

Der dritte Bereich der Internetkartographie kann als IP-Adressen- bzw. DomainNamen-Kartierung beschrieben werden. Gemeint sind damit alle Ansätze, bei denen die topographische Verteilung von IP-Adressen oder Domain-Namen untersucht wird. Übliche Fragestellungen zielen unter anderem auf die Häufigkeit von IP-Adressen oder Domain-Namen innerhalb einer räumlichen Einheit oder auf ihre Verteilung im Raum.

2 Im Internet sind zahlreiche weiterführende Informationen zum Thema Traceroute zu finden. Als Einstieg in die Thematik bietet sich die allgemeine Übersicht auf den Seiten http://www. traceroute.org an. 


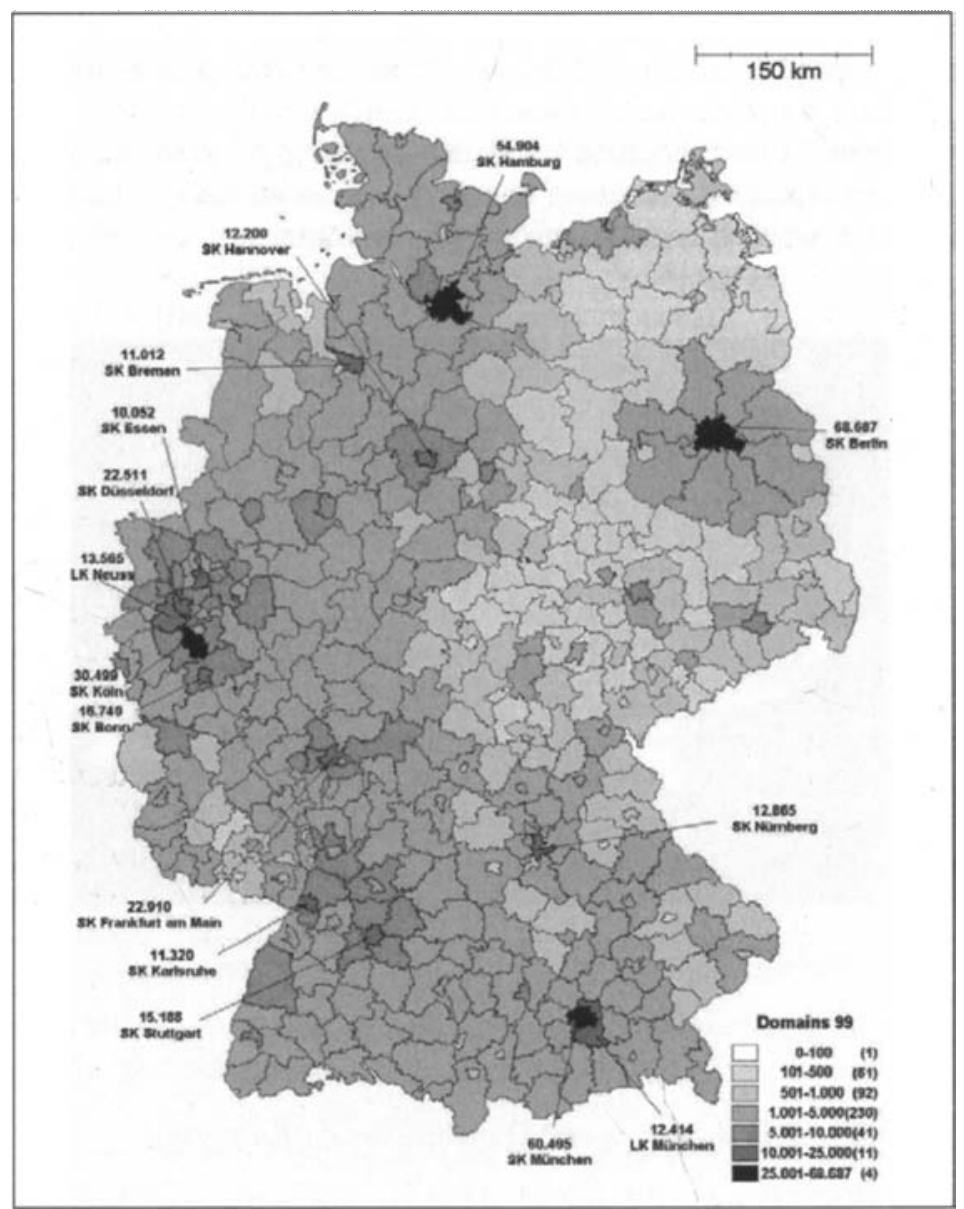

Abbildung 6: Verteilung der „.de“-Domains 1999 (Krymalowski 2002, S. 22).

Grundlage derartiger Analysen ist die Datenbasis der an die Internet Corporation for Assigned Names and Numbers (ICANN) angeschlossenen Organisationen, die für die Vergabe der Domain-Namen zuständig sind. Jede Organisation verfügt über eine öffentliche Datenbank, in der für jede Domain ihr Inhaber bzw. Administrator einschließlich seiner postalischen Adresse ausgewiesen wird. Alle deutschen „,de“-Top Level Domains (TLD) werden zum Beispiel von dem Unternehmen DENIC verwaltet (http://www.denic.de), auf dessen Homepage sich ein entsprechender Suchdienst für Einzelanfragen befindet. Der bereits erwähnte „Whois“-Dienst ermöglicht ebenfalls den Zugriff auf die Datenbank. Beispiele, bei denen Studien in Kooperation mit den Registrierungsorganisationen durchgefuihrt wurden, um die räumliche Verteilung der IP-Adressen zu ermitteln, findet man bei Krymalowski (2002) und Shiode und Dodge (1998) (vgl. Abb. 6).

In der Regel werden für derartige Analysen einzelne country code-Top Level 
Domains (ccTLD) herausgegriffen, wie zum Beispiel die bereits erwähnte ,.de“Domain für Deutschland. Generic-Top Level Domains (gTLD) wie „.com“, „.org“ und ,.net“, die auch unter den Namen der Inhaber in den untersuchten Ländern registriert sind, werden dagegen aufgrund des viel höheren Aufwands nicht mit einbezogen. Für diese Art der Kartierung gibt es keine speziellen Programme. Zur Kartierung werden in der Regel herkömmliche GIS-Anwendungen herangezogen, die die ermittelten Daten verarbeiten. ${ }^{3}$

\subsection{Kartierung des Internetzugangs}

Der vierte Typus der Internetkartographie umfasst alle Darstellungen, die sich mit der Frage der Zugangsmöglichkeiten zum Internet beschäftigen. Mit der Kartierung des Internetzugangs werden die Internetnutzer stärker in die Analyse mit einbezogen. Untersucht werden z.B. die Häufigkeit von internetfähigen PCs pro räumlicher Einheit, der PC-Anteil an der Bevölkerung, die Anzahl der Nutzer pro $\mathrm{PC}$, die Art des Internetzugangs (privater PC, Internetcafé, Arbeitsplatz), die verfügbare Bandbreite oder die Kosten des Internetzugangs. Im Gegensatz zur Kartierung der physischen Makrostrukturen des Internets richtet sich das Interesse im Fall der Kartierung des Internetzugangs auf die Mikrostrukturen der Anwenderebene. Auch bei diesem Ansatz besteht das Hauptproblem weniger in der Methodik der Darstellung als in der Erhebung der relevanten Daten.

Bis vor kurzem wurden noch Karten erstellt, die die Verfügbarkeit des Internets in allen Ländern der Erde zeigten. Da es mittlerweile nur noch wenige Länder gibt, die als not connected gelten, werden derartige Karten aber zunehmend obsolet und sind allenfalls für historische Untersuchungen zum Prozess der Ausbreitung des Internets interessant. Um heute die Disparitäten in der weltweiten Verbreitung des Internets aussagekräftig darzustellen, werden für aktuelle Karten im Maßstab der Nationalstaatenebene Indikatoren wie Bits pro Einwohner, Anzahl der Nutzer pro Internetanschluss oder Kosten des Internetzugangs verwendet. ${ }^{4}$ Das kanadische International Development Research Centre (IDRC, http://www.idrc.ca) hat solche Internet-Indikatoren für den afrikanischen Kontinent untersucht und in Form einer Kartensammlung publiziert.

Eine dieser Karten zeigt z.B. das afrikanische Pro-Kopf-Datenaufkommen in Verbindung mit dem Pro-Kopf-Bruttosozialprodukt sowie die Zielländer der von Afrika ausgehenden Internetverbindungen:

3 Detaillierte Informationen zu den Grundlagen der IP-Adressen-/Domain-Namen-System finden sich bei der Internet Corporation for Assigned Names and Numbers (ICANN, http://www. icann.org) und der Internet Assigned Numbers Authority (IANA, http://www.iana.org). Einen guten Einstieg vermitteln auch die Arbeiten von Asirvatham und Ravi (2001) und Mladenic (1998), die darüber hinaus in die Problematik einführen, wie auch funktionale Aspekte bei der kartographischen Analyse berücksichtigt werden können.

$4 \quad$ Einen Einblick in die weltweite Verbreitung des Internets liefert Crepin-Leblond (2000) anhand verschiedener interaktiver Karten unter http://www.nsrc.org/codes/bymap/world. html. 


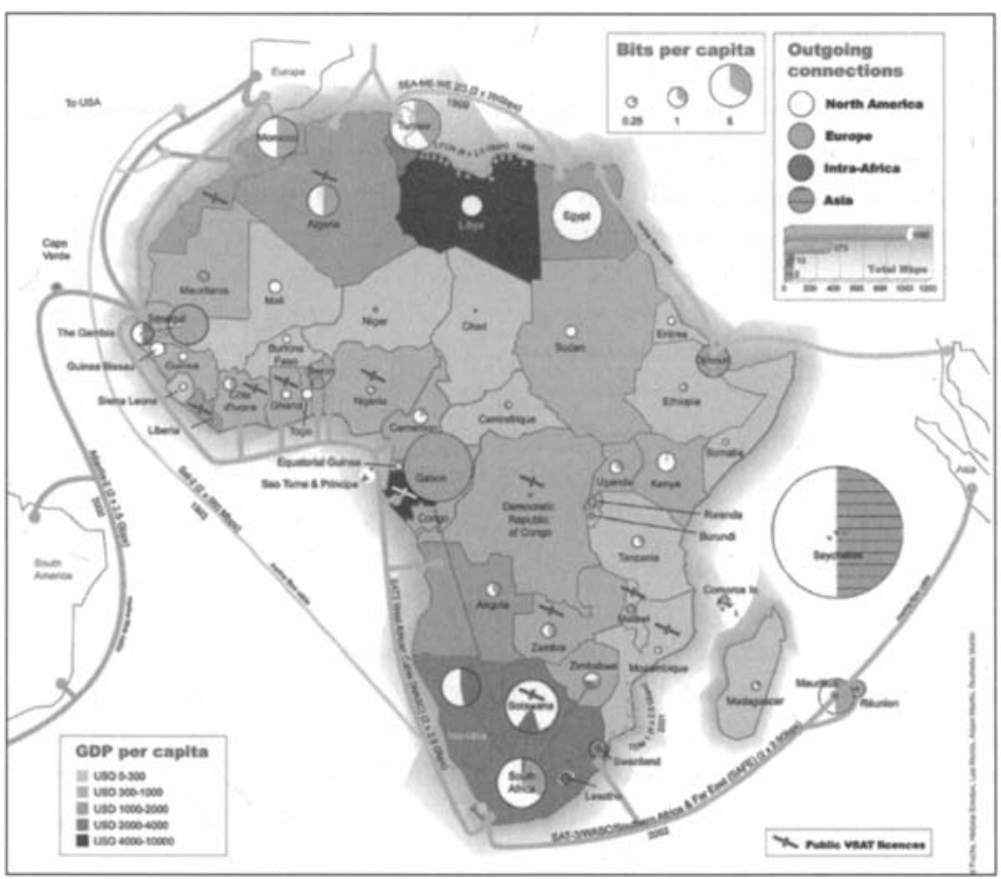

Abbildung 7: Die afrikanische Internet-Infrastruktur in Zusammenschau mit dem Pro-Kopf-BSP und dem Pro-Kopf-Datenaufkommen 2002 (International Development Research Centre 2003).

Vergleichbare Analysen können auch auf städtischer Ebene durchgeführt werden, so geschehen in einer Studie von Jeremy Crampton, der die geographische Dimension des Digital Divide in Atlanta, Georgia, mit Hilfe entsprechender Karten dargestellt hat (Crampton 2001). ${ }^{5}$

\section{ANWENDUNG UND NUTZEN DER INTERNETKARTOGRAPHIE}

In den vorangegangen Abschnitten ist deutlich geworden, dass die Internetkartographie viele unterschiedliche Bereiche untersucht. Entsprechend vielfältig sind auch die Anwendung und der Nutzen der Internetkartographie. Es lassen sich zwei Anwendergruppen unterscheiden: Diejenigen Anwender, die sich in der Hauptsache auf technische Aspekte des Internets beziehen, und jene, die die

5 Weiterführende Arbeiten, die sich mit verschiedenen Aspekten der Kartierung statistischer Zugangsdaten beschäftigt haben, finden sich auf den Seiten von Zooknic, einem Projekt von Matthew Zook zur Erfassung der Strukturen des Internets mittels unterschiedlicher Messund Analyseverfahren. Auf der Homepage sind auch verschiedene Kartenbeispiele verfugbar (http://www.zooknic.com). 
Auswirkungen des Internets auf die Gesellschaft und ihre Umwelt untersuchen.

In der Anfangszeit des Internets war die kartographische Darstellung der technischen Aspekte ein attraktives Mittel, um die räumlichen Distanzen sichtbar zu machen, die das neue Kommunikationsmittel überbrücken konnte. Auch heute noch sind aus Informations- und Marketinggründen auf den Homepages fast aller Netzbetreiber Karten der jeweiligen technischen Internet-Infrastruktur zu finden. Firmen, die Internet-Performanceanalysen durchführen, setzen ebenfalls kartographische Mittel ein, um Schwachstellen oder Bereiche hohen Datenaufkommens für ihre Kunden zu visualisieren. Der Einsatz von Internetkarten im technisch orientierten Bereich ist aber in der Regel auf veranschaulichende Zwecke begrenzt.

Für die Anwender der Internetkartographie, die die Einflüsse der Internettechnologie auf die Gesellschaft bzw. die Menschen untersuchen, sind die Karten hingegen von ganz zentraler Bedeutung. Die Karten dieser Anwender können wieder nach ihren Inhalten unterschieden werden, d.h. danach, welche Dimension der Beziehung Gesellschaft-Mensch-Internet jeweils beleuchtet wird. $\mathrm{Zu}$ den wichtigsten inhaltlichen Dimensionen gehören die sozio-demographische, die wirtschaftliche und die politisch-rechtliche Dimension. Im Folgenden soll gezeigt werden, welchen Nutzen die Internetkartographie bei der Analyse dieser drei Aspekte des Gesellschafts-Mensch-Internet-Verhältnisses haben kann.

\subsection{Soziodemographische Struktur der Internetnutzer}

Seitdem sich die Gruppe der Internetnutzer nicht mehr ausschließlich auf männliche Akademiker unter 30 Jahren beschränkt und solange noch nicht jede einzelne Person über einen Internetanschluss verfügt, stellt sich die Frage, wer die Nutzer des Internets sind (vgl. hierzu auch den Beitrag von LanghagenRohrbach in diesem Buch).

Die bisherige Entwicklung der Nutzung des Internets zeigt, dass die mit ihm verbundenen Möglichkeiten bisher nur von einem Teil der Bevölkerung wahrgenommen werden (können). Seitdem diese ungleiche Nutzung bekannt ist, gehen so genannte Digital Divide-Studien gezielt der Frage nach, welche Gruppen bisher von der Nutzung des Internets ausgeschlossen sind. Derartige Analysen führen sowohl auf lokaler als auch auf globaler Ebene zu dem Ergebnis, dass es Korrelationen zwischen bestimmten ökonomischen und demographischen Merkmalen der Nutzer und der Art der Nutzung des Internets gibt. Die Internetkartographie kann in dieser Hinsicht dazu beitragen, diejenigen Faktoren zu ermitteln, die über den Zugang zum Internet entscheiden.

Eine groß angelegte Studie des US-amerikanischen Handelsministeriums aus dem Jahr 2000 hat z.B. ergeben, dass sich die Unterschiede in der Nutzung durch verschiedene gesellschaftliche Gruppen mit zunehmender Verbreitung des Internets zwar verringern, dass es aber trotzdem noch immer Gruppen gibt, die das Medium deutlich seltener nutzen. Dazu gehören u.a. ethnische Minderheiten, ältere Generationen, einkommensschwache Haushalte und allein Erziehende. Auch 
Bewohner ländlicher Gebiete nutzen das Internet noch immer seltener als Stadtbewohner (vgl. National Telecommunications \& Information Administration 1999). Ähnliche Verteilungsmuster zeigt der internationale Vergleich: Das Internet ist am stärksten in den führenden Wirtschaftsnationen verbreitet.

\subsection{Wirtschaftliche Dimension des Internets}

Mit seiner rasanten Verbreitung und der damit einhergehenden Kommerzialisierung hat das Internet in den letzten Jahren einen ,finanziellen Hype“ ausgelöst. Trotz dieser Entwicklung hat die propagierte Entkoppelung der Wirtschaft vom Raum nicht stattgefunden. Das Internet kann zwar dabei helfen, Kommunikationsdistanzen zu überbrücken, nach wie vor fallen jedoch Standortfaktoren wie die Nähe zu Geschäftspartnern, das Angebot an Dienstleistungen und qualifizierten Arbeitskräften oder auch die Verkehrsinfrastruktur stark ins Gewicht. Deswegen haben sich die Wirtschaftszentren in den letzten Jahren räumlich weder im nationalen noch im internationalen Maßstab entscheidend verlagert. Vielmehr etabliert sich das Internet gerade dort, wo ohnehin bereits eine wirtschaftliche Aktivität vorhanden ist. Egal, welche Art der Darstellung man herausgreift, sobald es um quantitative Aspekte des Internets und ihre räumliche Dimension geht, erhält man fast automatisch ein Bild, das sich mit der Verteilung der wirtschaftlichen Aktivität vor allem des tertiären Sektors, in Teilen auch des sekundären Sektors, deckt. Die Verbreitung des Internets wird damit zu einer Art Indikator für wirtschaftliche Aktivität. Mittels Internetkarten, die die Verbreitung des Mediums zeigen, können dann wirtschaftlich aktive Regionen ermittelt werden. Auf diese Weise können im globalen Maßstab z.B. Zentren der Globalisierung, im nationalen Maßstab wirtschaftlich aktive Regionen identifiziert werden. Um Internetkarten zu diesem Zweck noch gewinnbringender einsetzen zu können, müssten allerdings zunächst die Strukturen der Internetnutzung im wirtschaftlichen Bereich systematisch untersucht werden. Bisher konzentrierte man sich allenfalls auf einzelne Branchen wie das Bankwesen oder auf ausgewählte Regionen. Allgemein gültige Aussagen können auf dieser Grundlage nicht getroffen werden.

Dennoch kann die analytische Verwendung von Internetkarten schon heute aufschlussreich sein. So zeigt sich etwa für Afrika, dass das Pro-Kopf-Datenaufkommen und die Höhe des Bruttosozialproduktes nicht in allen Ländern korrelieren (vgl. Abb. 7). Beispielsweise sind die Seychellen durch ein überdurchschnittlich hohes Datenaufkommen gekennzeichnet, während Libyen, das ebenfalls über ein vergleichsweise hohes BSP verfügt, ein relatives geringes ProKopf-Datenaufkommen aufweist. Vergleicht man die Wirtschaftsstruktur der beiden Länder, stellt man fest, dass ein Großteil der libyschen Einnahmen aus dem Ölgeschäft stammt, während auf den Seychellen der Tourismus die Haupteinnahmequelle ist. Die Zusammenführung der beiden Faktoren lässt daher den Schluss zu, dass die Tourismusbranche in hohem Maße Gebrauch vom Internet macht, um ihre Dienstleistungen anzubieten, während dieser Faktor in der Ölin- 
dustrie nur eine geringe Rolle spielt. Leider gibt es kaum vergleichbare Kartenbeispiele anderer Regionen, es fehlt eben gerade in diesem Bereich noch an Grundlagenforschung.

\subsection{Die politisch-rechtliche Dimension des Internets}

Die politisch-rechtliche Dimension des Internets umfasst ein großes Themenspektrum, aus dem einige Aspekte bereits in den beiden vorangegangenen Abschnitten angesprochen wurden. Beispielsweise kann das Phänomen des Digital Divide sowohl in einem demographischen als auch in einem politischen Kontext betrachtet werden. Vielfach überschneiden sich die Bereiche, wenn zum Beispiel als Folge ökonomischer oder demographischer Analysen politische Steuerungsinstrumente eingesetzt werden. Projekte wie Schulen ans Netz (http:// www.schulen-ans-netz.de) haben zu einer bundesweit flächendeckenden Verfügbarkeit des Internets an Schulen beigetragen. Der Nutzen der Internetkartographie entsteht in diesem und vergleichbaren Fällen daraus, dass räumliche Disparitäten der Internetnutzung aufgedeckt werden, woran dann politische Fördermaßnahmen anschließen können.

Auf einer anderen Ebene stellt sich die Frage, welchen Einfluss die Internettechnologien auf das Gefüge der Nationalstaaten haben werden. Das Internet wird durch staatliche Grenzen nicht beschränkt. Daher konnten sich viele rechtliche Grauzonen herausbilden, in denen die herkömmlichen staatlichen Ordnungssysteme nicht greifen. Vor allem die Bereiche Besteuerung, Urheber- und Markenrecht sowie die Möglichkeiten der grenzüberschreitenden Strafverfolgung sind von dieser Problematik betroffen. Wie schwer sich die Politik tut, in diesen Fraugen einen Konsens zu finden, zeigt der Prozess der Verabschiedung der sogenannten Cybercrime Convention im Europarat. Immer wieder tritt der Konflikt zwischen effektiver Bekämpfung von Kriminalität und dem gleichzeitigen Festhalten an nationalstaatlicher Souveränität deutlich zu Tage (vgl. Rötzer 2001). In der Internetkartographie gibt es bislang keine Arbeiten, die sich diesem Themenbereich widmen. Es liegen lediglich Darstellungen aus angrenzenden Bereichen vor, wie etwa die Erfassung und Visualisierung der räumlichen Dimension der organisierten Kriminalität. Dennoch sind auch in der Internetkartographie Ansätze denkbar, um den Einfluss der genannten Entwicklungen auf den Aktionsraum der einzelnen Staaten zu verdeutlichen. Während dieser in Bezug auf die Strafverfolgung bisher weitgehend deckungsgleich mit den staatlichen Grenzen war, kann es durch internationale Abkommen (etwa zur Bekämpfung von Internetdelikten) zu einer Ausdehnung dieses Rechtsraumes kommen. Auch die Frage nach dem Geltungsbereich nationalen Rechts muss in Zukunft wahrscheinlich neu und anders beantwortet werden. In den USA wird seit geraumer Zeit nach einem rechtlichen Mittel gesucht, um die Betreiber der Internettauschbörse Kazaa vor ein amerikanisches Gericht zu bringen, obwohl die Firma auf der Pazifikinsel Vanuatu registriert ist und ihren Hauptsitz in Australien unterhält. Ein kalifornisches Gericht hat erst kürzlich eine Klage gegen Kazaa mit der Begründung 
zugelassen, dass eine große Anzahl kalifornischer Bürger den Kazaa-Client heruntergeladen und benutzt hätten (Heise Newsticker 2003). Der Ausgang dieses Prozesses ist momentan noch offen. Dasselbe Gericht hat bei einem vergleichbaren Prozess die Betreiber zweier anderer Tauschbörsen freigesprochen, so dass es nicht unwahrscheinlich erscheint, dass es auch in diesem Prozess zu keiner Verurteilung kommt. Trotzdem wurde durch dieses Urteil erstmals die Möglichkeit geschaffen, ein Internetangebot gerichtlich nicht nach dem Recht des Ursprungslandes, sondern nach den Bestimmungen des Abnehmerlandes zu beurteilen. Es bleibt abzuwarten, ob dieser Fall eine Ausnahme bleibt oder sich eine neue Praxis etabliert. Sollte letzteres der Fall sein, wird die Internetkartographie interessante Aufschlüsse darüber liefern können, wie sich der Prozess der Ausdehnung nationalen Rechts vollziehen wird.

\section{INTERNETKARTOGRAPHIE UND GEOGRAPHIE}

Die Stationen dieses Aufsatzes haben gezeigt, dass die Verortung des Internets mittels kartographischer Methoden in vielfältiger Weise durchgeführt wird und dass die unterschiedlichsten Aspekte des neuen Mediums in ihrem physischräumlichen Bezug beleuchtet werden können. So vielfältig die Anwendungsmöglichkeiten der Internetkartographie theoretisch auch sein mögen, zum jetzigen Zeitpunkt befindet sie sich noch in einem sehr frühen Entwicklungsstadium und ein Großteil ihres Potenzials ist noch nicht ausgeschöpft. Ein Grund mag darin liegen, dass man für die Erstellung derartiger Karten die entsprechenden geographisch-kartographischen Kenntnisse haben muss, um den räumlichen Faktor in die Fragestellung mit einzubeziehen. Andererseits ist es nicht möglich, ohne die Kenntnis der technischen Grundlagen der Informationstechnologie den Rahmen potenzieller Anwendungsgebiete abzustecken.

In der wissenschaftlichen Praxis findet man nur wenige Berührungspunkte der Disziplinen Geographie und Informatik. Eine Annäherung findet erst in den letzten Jahren statt, seitdem sich GIS-Anwendungen zunehmend verbreiten und die Nutzung von PC und Internet aus der geographischen Forschung nicht mehr wegzudenken ist. Der immer häufiger anzutreffende Studiengang Geoinformatik zeigt, dass die Kooperation der beiden Disziplinen zu gewinnbringenden Ergebnissen führen kann. Für die Internetkartographie kann die Geographie das Wissen der Informatik bezüglich der technischen Grundlagen der Informationstechnologie und der Möglichkeiten der Datenverarbeitung Gewinn bringend nutzen. Sie ist in der Lage, die rein technischen Daten mit Aspekten des physischen oder sozialen Raumes zu relevanten Fragestellungen zu verbinden. 


\section{LITERATUR}

Asirvatham, Arul Prakash und Kranthi Kumar Ravi (2001): Web Page Categorization Based on Document Structure, online: http://gdit.iiit.net/ arul/paper.pdf

Computer History Museum (1983): Internet Topography Map, online: http://computerhistory.org/exhibits/internet_history/full_size_images/1983_topo. gif

Cox, Kenneth C. u. a. (1996): 3D Geographic Network Displays, online: http://citeseer.nj.nec.com/cache/papers2/cs/1233/http:zSzzSzwww.bell-labs.ComzS zuser zSzeickzSzbibliographyzSz1996zSz3D_copyright.pdf/cox96geographic.pdf

Crampton, Jeremy W. (2001): The Geography of the Digital Divide, online: http://monarch.gsu.edu/jcrampton/digital-divide

Crepin-Leblond, Olivier M. J. (2003): International E-mail Accessibility, online: http://www.nsrc.org/codes/country-codes.html

Heise Newsticker (2003): Kazaa kann in den USA verklagt werden, online: http://www.heise.de/newsticker/data/uma-11.01.03-003

International Development Research Centre (2003): Map of „The Internet: Out of Africa“, online: http://network.idrc.ca/uploads/user-S/10439608991map.GIF

Krymalowski, Mark (2002): Die regionale Verteilung von Domainnamen in Deutschland, online: http://www.denic.de/DENICdb/stats/da_krymalowski.pdf

Mladenic, Dunja (1998): Turning Yahoo into an Automatic Web-Page Classifier, online: http://www-ai.ijs.si/DunjaMladenic/papers/PWW/pwwECAI98yr.ps

National Telecommunications \& Information Administration (1999): Americans in the Information Age Falling Through the Net, online: http://www.ntia.doc.gov/ntiahome/digitaldivide/index.html

Papadakakis, Nektarios u. a. (1998): Palantir: A Visualization Tool for the World Wide Web, online: $\mathrm{http}: / /$ archvlsi.ics.forth.gr/html_papers/INET98_Palantir

Rademacher, Cay (2001): Das Netz der Netze, in: Geo, Nr.3, S. 73.

Rötzer, Florian (2001): Soll illegales Hosting ein Verbrechen werden, online: http://www.heise.de/tp/deutsch/inhalt/te/7480/1.html

Salus, Peter (1995): Casting the Net: From ARPANET to INTERNET and beyond..., Reading.

Shiode, Narushige und Martin Dodge (1998): Using GIS to Analyse the Spatial Pattern of the Internet in the United Kingdom,

online: http://www.geog.ucl.ac.uk/casa/martin/internetspace/paper/gisruk98.html

Sprint (2002): European IP Backbone, online: http://www.sprintworldwide.com/english/maps/europe.pdf

Teaching to Change LA (2001): G.I.S. Mapping at Jordan High, online: http://tcla.gseis.ucla.edu/divide/politics/maps/internethome.html

Yook, Soon-Hyung (2001): Modeling the Internet's Large-Scale Topology, online: http://arxiv.org/pdf/cond-mat/0107417 



\section{INTERNET UND INTERNET-USER \\ WER NUTZT DAS NETZ WO? \\ Christian Langhagen-Rohrbach}

\section{EINLEITUNG}

Moderne Telekommunikationsmittel erwecken häufig den Anschein, durch die Übertragung von Daten in Sekundenschnelle über große Distanzen hinweg sei der Standort einzelner Personen oder Institutionen weniger wichtig geworden. So besagt auch die immer wieder diskutierte These vom "Ende der Geographie" (Mosco 2000) oder dem ,,death of distance“ (Cairncross 1997), dass durch die zunehmende Nutzung moderner Informations- und Kommunikationstechnologien die Bedeutung physisch-räumlicher Kategorien sinkt, vor allem für Unternehmen bzw. wirtschaftliche Aktivitäten. Die Idealvorstellungen gehen von einer Substituierung des Verkehrs durch Kommunikation, vom Ersatz persönlicher durch mediale Kontakte oder einer Auflösung des bestehenden Siedlungsgefüges in die Fläche aus. Ubiquitär angenommene Netzanschlüsse sollen zu einer stark steigenden Bedeutung weicher Standortfaktoren und damit einem neuen Gefüge der Wohnund Unternehmensstandorte führen.

Entsprechend wenig Aufmerksamkeit wurde in den bislang vorliegenden Untersuchungen den Standorten derjenigen gewidmet, die die modernen Kommunikationsmedien nutzen. Meist steht nur „das“ Internet im Vordergrund - womit auch unterschlagen wird, dass die Bezeichnung „Internet“ im Grunde nur ein Oberbegriff ist, der verschiedene Dienste zusammenfasst. ${ }^{1}$ Doch sind die $\mathrm{Zu}$ gangsmöglichkeiten zum Internet und seinen Diensten tatsächlich überall gegeben? Und welche Zusammenhänge bestehen zwischen ihnen und der soziodemographischen Struktur der Internetnutzer? Mit dem Ziel, die genannte These vom Ende der Geographie kritisch zu hinterfragen, wird daher in diesem Aufsatz folgender Leitfrage nachgegangen: Wer nutzt das Internet von wo aus und zeigen sich bestimmte regionale Verteilungsmuster in der Internetnutzung?

Dabei ist die Nachfrage berechtigt, wozu die Kenntnis der regionalen Verteilung der Internet-Nutzer überhaupt sinnvoll sein soll. Das Spektrum der möglichen Antworten auf diese Frage ist breit: Es reicht vom Interesse an der Suche

Die populärsten Dienste sind das sog. Worldwide Web (WWW), in dem mit Hilfe der Browser Informationen über das Netz abgerufen werden können, und die elektronische Post (e-Mail). Daneben gibt es den Chat, die Dateiübertragung $(F T P$ ) oder die Diskussionsforen des Usenet (Newsgroups). Diese Dienste sind es, die die Kommunikation über das Internet erst möglich machen. Zur Nutzung ist ein Zugang zum Internet über einen Internet Service Provider (ISP) notwendig. 
nach regionalen Unterschieden in der Nutzung und deren Ursachen bis hin zu der Möglichkeit, über eine Regionalisierung der Nutzer auch sog. Geomarketing zu betreiben. Auch für virtuelle Rathäuser oder allgemeiner E-Government-Lösungen ist es vonnöten zu wissen, welche Nutzer des Internets es bislang gibt, wo noch Potenziale schlummern und wie diese ggf. nutzbar gemacht werden könnten. $^{2}$

Nach Castells (2001, S. 208-210) können die räumlichen Ausprägungen des Internets in drei Kategorien eingeteilt werden:

- Die „technical geography“ umfasst vor allem die betriebsnotwendige technische Infrastruktur, also Datenleitungen, aber auch IP-Adressen sowie den Besitz von Domain-Namen,

- die „economic geography of Internet production“ umfasst Unternehmen, die für das Internet produzieren, so dass in diese Kategorie vornehmlich die Forschung an bzw. die Entwicklung technischer Innovationen fallen, und

- die „spatial distribution of its users“, die sich mit der Lokalisierung der Internet-Nutzer befasst.

Insbesondere die dritte Kategorie ist hier Gegenstand näherer Betrachtung. In mehreren Untersuchungen wurden bislang Daten vorgelegt, die sich mit der Nutzung des Internets nach Nationen befassen. Als gesichert kann in diesem Zusammenhang gelten, dass es eine „digital divide“ gibt, die die entwickelten Nationen der nördlichen Hemisphäre von der südlichen trennt. Sowohl in Afrika als auch in Asien und Südamerika liegen die Nutzerzahlen relativ wie absolut unter denen der Staaten auf der Nordhalbkugel (vgl. dazu Zook 2001). Während die weltweite Verteilung der Nutzer recht gut bekannt ist, fehlen vor allem aus Europa Untersuchungen, die sich mit der Nutzerverteilung auf regionaler Ebene befassen. Zu den soziodemographischen Merkmalen der Nutzer sind zahlreiche Befragungen durchgeführt worden. Da aber allgemein angenommen wurde, das Internet würde zu einem Bedeutungsverlust physisch-räumlicher Kategorien und Verteilungen führen, wurden die Standorte der Nutzer nur selten - und dann nur recht großräumig - erfasst. So liegen bislang auch nur wenige Erkenntnisse über regionale Differenzierungen der Internet-Nutzung vor. Castells (2001, S. 212) ist der Ansicht, dass die Intensität der Nutzung in Stadtregionen am höchsten sei, nennt aber für diese Vermutung keine empirischen Belege.

Dieser Aufsatz versucht, die räumliche Struktur der Internet-Nutzung in Deutschland näher zu beleuchten, und will darlegen, ob auch innerhalb einer Nation Nutzungsunterschiede sichtbar gemacht werden können. Der Unterschied zwischen Zonen unterschiedlich starker Internet-Nutzung könnte in diesem Zusammenhang als Indikator für die Innovationsfreudigkeit von Regionen dienen

2 Zu virtuellen Rathäusern und der Problematik des Internets in der Kommune vgl. den Beitrag von Floeting in diesem Band. 
und indirekt Aufschluss auch über die vorhandenen endogenen Potenziale dieser Teilräume geben.

\section{NUTZUNGSVORAUSSETZUNGEN}

Zur Nutzung des Internets ist zuerst eine entsprechende Infrastruktur vonnöten, mit deren Hilfe auf die in diesem Medium verfügbaren Informationen zugegriffen werden kann. Neben den Unternehmen, die diese Infrastruktur betreiben und zur Verfügung stellen, sind Anbieter von Informationen notwendig. Dabei handelt es sich häufig um Firmen, aber auch um Privatpersonen.

Das Angebot an Datenleitungen ist über die betreffenden Firmen zu recherchieren. Entsprechende Karten sind z.B. in Dodge/Kitchin (2001, S. 31) oder in Langhagen-Rohrbach (2001, S. 425) enthalten. Diese zeigen eine hohe Netzdichte vor allem in und um die Zentren der jeweils untersuchten Nationen und besonders leistungsfähige Verbindungen zwischen den hochrangigen Zentren (vgl. Abb. 1).

Die für den technischen Betrieb des Internets erforderlichen Internet-Protocol (IP)-Adressen werden ebenfalls gruppenweise an Internet Service Provider (ISP) vergeben und sind recherchier- und kartierbar. Eine entsprechende Analyse, die eine deutliche Konzentration von IP-Adressen in den Verdichtungsräumen Großbritanniens nachweist, haben Shiode und Dodge (1999) vorgelegt.

Auch die Anbieter von Internet-Inhalten lassen sich noch relativ einfach ausfindig machen, da sie für ihr Angebot meist eindeutige sog. Domain-Namen verwenden, die zentral in Network Information Centern verwaltet werden. Diese registrieren zu jeder Domain Einträge, die den Besitzer der Domain beschreiben und Rückschlüsse über seine Verortung zulassen. Die Registrierung eines Domain-Namens kann als Hinweis darauf verstanden werden, dass der Besitzer beabsichtigt, unter dem beantragten Namen ein Angebot im Internet verfügbar zu machen. Entsprechende Untersuchungen kommen auch hier zu dem Ergebnis, dass in Verdichtungsräumen besonders viele Domain-Namen registriert sind (vgl. Krymalowski 2000 oder Langhagen-Rohrbach 2002).

Die vorhandenen Datenleitungen, die von den ISP verwendeten IP-Adressen und die Domain-Namen weisen jeweils in hoch verdichteten Regionen hohe Konzentrationen auf und können als die Angebotsseite des Internets verstanden werden. 


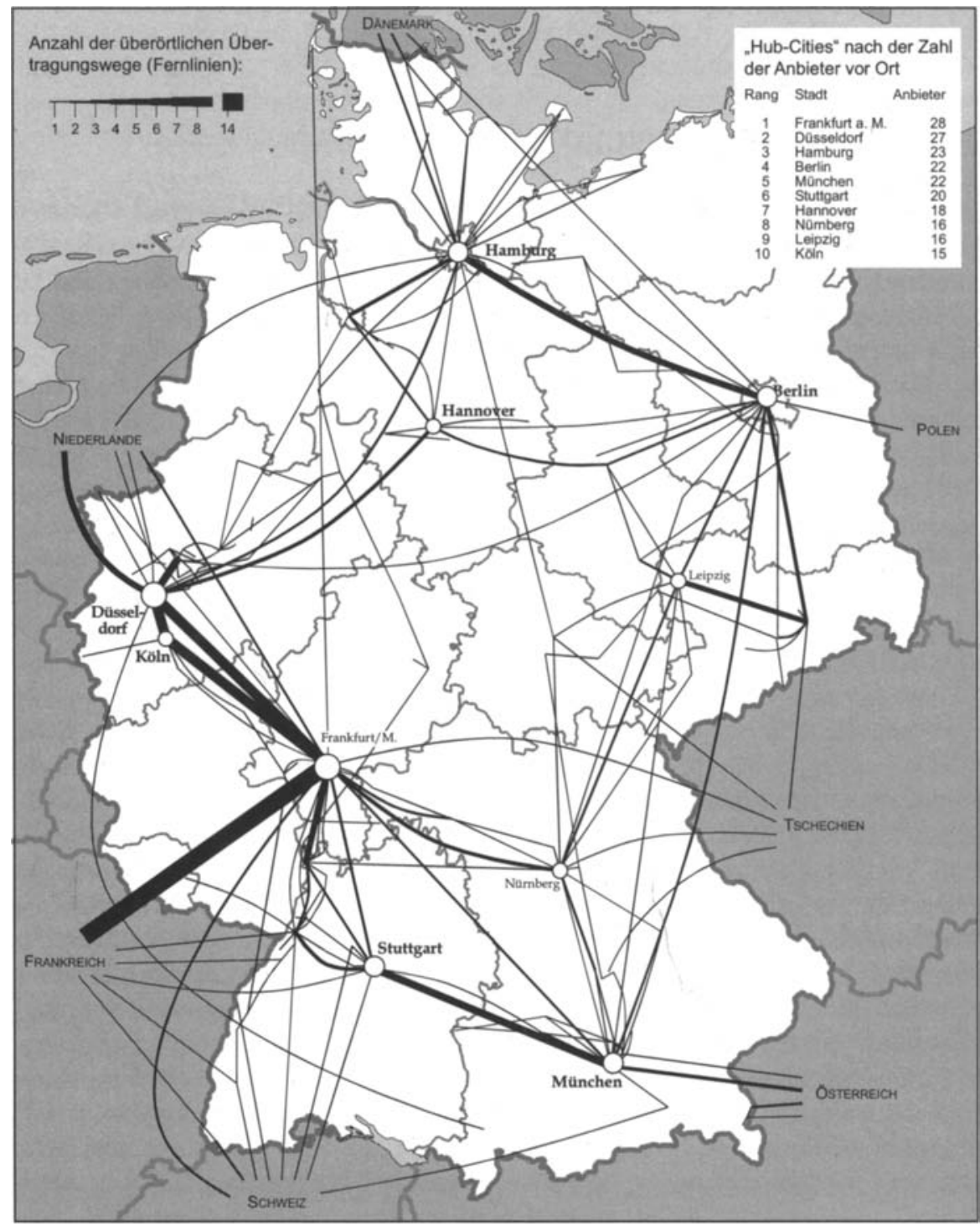

Abbildung 1: Telekommunikationsinfrastruktur in Deutschland 2003 (Quelle: Liste der von der Regulierungsbehörde für Post und Telekommunikation (Reg TP) erteilten Übertragungsweglizenzen, Klasse 3, Stand März 2003). 


\section{DIE NUTZER DES INTERNETS IM ALLGEMEINEN}

Schwieriger ist es, Daten über die regionale Verteilung der Internetnutzer, also über die Nachfrageseite des eben beschriebenen Angebots, zu bekommen. Ein erstes Problem entsteht dadurch, dass der Zugang zum Internet einfacher ist als das Anbieten von Informationen in diesem Medium. So gibt es allein in Deutschland mehrere Dutzend ISP, die ihren Kunden einen Internet-Zugang zur Verfügung stellen. Für jede dieser Firmen stellt der Kundenstamm eine wichtige Ressource dar, über die keine Auskünfte nach außen gegeben werden - dies gilt für kleine Anbieter ebenso wie für bundesweit tätige. Damit ist es kaum möglich, Internet-Nutzer direkt zur Nutzung dieses Mediums zu befragen. Dabei wäre eine konsequente Untersuchung dieser Klientel dringend nötig, um - auch aus finanziellen Interessen - zu klären, welche Bevölkerungsgruppen über dieses Medium zu erreichen sind, da davon auch abhängt, welche Dienste in unserer Gesellschaft virtualisiert werden können: Stellt sich heraus, dass große Bevölkerungsgruppen offline sind, so wäre E-Government ein exklusives Angebot für eine ganz bestimmte Klientel, so dass der Nutzen dieses Angebotes neu überdacht werden müsste. Entsprechendes gilt für Online-Shopping und Werbung im Internet.

Daher werden seit Jahren Befragungen unter den Internet-Usem durchgeführt: Diese beruhen jedoch auf dem Prinzip der Freiwilligkeit und untersuchen nur eine zufällige Auswahl: Befragt werden nur solche Surfer, die den entsprechenden Fragebogen im Netz aufrufen (z.B. im Rahmen der W3B-Studien oder der Untersuchung der Initiative „Perspektive Deutschland“( ${ }^{\star 3}$ ). Um möglichst viele Probanden für die Untersuchungen zu gewinnen, werden Anreize geschaffen - z.B. in Form von Gewinnspielen, die an die Befragung gekoppelt werden. Die Repräsentativität dieser Untersuchungen beruht dabei allein auf der großen Zahl an Teilnehmern, ohne dass ausgeschlossen werden kann, dass dieselbe Person mehrfach an einer solchen Untersuchung teilgenommen hat. Zudem verzichten diese Untersuchungen häufig auf eine Regionalisierung bzw. die geringe Zahl der erfassten Personen würde keine Regionalisierung ermöglichen. Schließlich werden Studien auch irrefuihrend betitelt: So verbirgt sich hinter den Angaben zur „Regionalen Online-Nutzung“ bei ComcultResearch (2002) lediglich eine Untersuchung, die danach fragt, inwiefern Internetinhalte zum eigenen Wohnort bzw. der Wohnregion genutzt werden - eine Differenzierung danach, wie groß die Nachfrage aus den betreffenden Regionen ist, wird jedoch nicht vorgenommen.

Eine Ausnahme in diesem Zusammenhang stellt der ,(N)Onliner Atlas 2002“4 dar - für diese Studie wurden die Probanden nicht via Internet untersucht, sondern

3 Die Online-Befragung von Perspektive Deutschland mit rd. 170.000 Teilnehmem wurde anschließend mit Hilfe mathematischer Verfahren auf die Bevölkerungsstruktur der Bundesrepublik umgerechnet. Dabei half eine flankierend durchgeführte Telefonbefragung (Perspektive Deutschland 2002, S. 15-23).

4 Ich danke der Firma EMNID und insbesondere Frau Nina Fluck für die freundliche Überlassung der Rohdaten des „(N)Onliner Atlas 2002" zur Auswertung. Die im Rahmen des „(N)Onliner Atlas 2002" vorgelegten (kartogtaphischen) Auswertungen beschränken sich auf 
es wurde per Telefon eine repräsentative Stichprobe der Bevölkerung befragt (insgesamt 30318 Befragte). Damit wurden nicht nur Internet-Nutzer (Onliner), sondern auch Nicht-Nutzer (Offliner) erfasst, so dass auch ein direkter Vergleich beider Gruppen möglich ist. Als dritte Gruppe wurden die sog. Intender, also die Personen ausgegliedert, die planen, das Internet in den nächsten 12 Monaten $\mathrm{zu}$ nutzen (vgl. EMNID 2002). Diese Kategorie muss jedoch hinsichtlich ihres tatsächlichen Aussagegehaltes mit Vorsicht betrachtet werden. Die Stichprobenauswahl erfolgte beim „(N)Onliner Atlas 2002" so, dass eine Regionalisierung der Befragungsergebnisse bis auf die Ebene der Regierungsbezirke möglich ist. Der Begriff der Internet-Nutzung beschränkte sich dabei auf die populärsten Dienste dieses Mediums, nämlich das sog. Worldwide Web und e-Mail, wobei das Surfen bzw. mailen maximal vier Wochen zurück liegen durfte.

\section{DIE INTERNET-NUTZER IN IHRER SOZIODEMOGRAPHISCHEN STRUKTUR}

Bevor die Struktur der Internet-Nutzer näher dargestellt wird, ist von besonderem Interesse, wie groß der Anteil der Internet-Nutzer an der Bevölkerung derzeit ist. Im Dezember 2002 waren ca. 40\% der deutschen Bevölkerung (insgesamt) online, entsprechend ca. 39 Mio. Personen: EMNID (2002) gibt $41,7 \%{ }^{5}$ Anteil an der Bevölkerung an, während NUA.IE (2003) von 38,9\% und die ARD/ZDF-OnlineStudie 2002 (van Eimeren u.a. 2002) von 44,1\% Onlinern (vgl. Abb. 2) ausgeht. Betrachtet man das Wachstum der Zahl der Onliner, so macht sich ab 2001 eine deutliche Verlangsamung des Wachstums bemerkbar, so dass eine gewisse Marktsättigung erreicht zu sein scheint (van Eimeren et al. 2002, S. 347). Dennoch ist die Zahl der Internet-Nutzer mittlerweile so groß, dass fast die Hälfte der Einwohner Deutschlands online ist und es stellt sich die Frage, welche Struktur die Gruppe der Internetnutzer in Bezug auf ihre soziodemographischen Merkmale hat - vor allem im Vergleich zu den Nichtnutzern.

die beschreibende Darstellung der erhobenen Sachverhalte, ohne dass die hinter den dargestellten Fakten zu vermutenden oder nachgewiesenen Entwicklungsprozesse dargestellt werden würden.

5 Alle Angaben der EMNID-Studie beziehen sich auf die Bevölkerung über 14 Jahre, da es aus rechtlichen Gründen nicht möglich ist, auch jüngere Personen zu befragen. 
Onliner in Mio.

Anteil an der Bevölkerung in \%

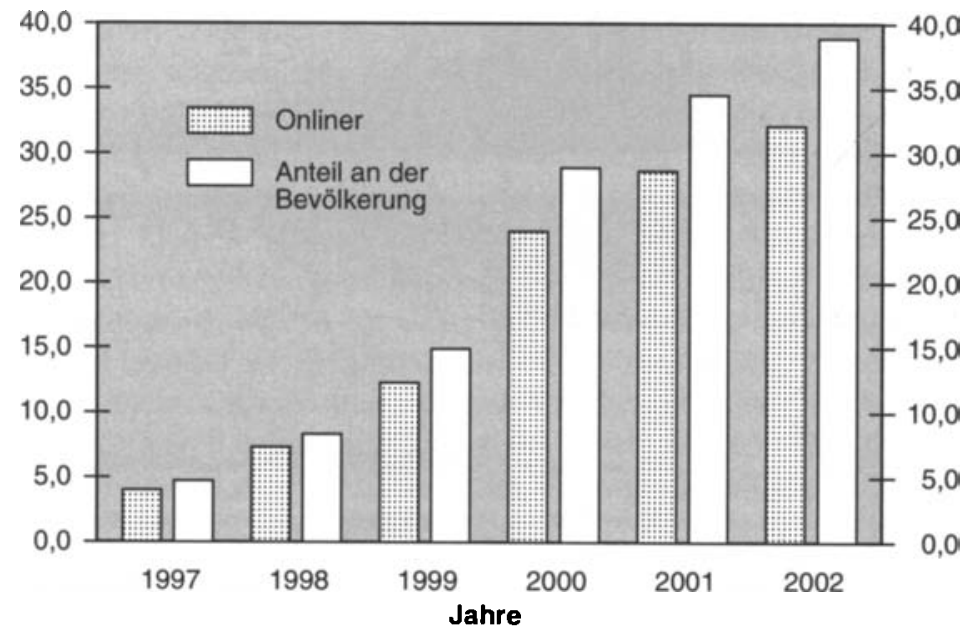

Abbildung 2: Entwicklung der Onliner seit 1997 (Quelle: eigene Abbildung nach W3B, EMNID 2002).

In den frühen 1990er Jahren, als die starke Verbreitung des Internets mit dem Aufkommen der Browser begann, ließ sich der typische Internet-Nutzer noch mit den Attributen männlich, unter 30 Jahre und Student beschreiben. So lag der Anteil der Männer in den ersten Untersuchungen der Nutzerstruktur des Internets deutlich über 90\% (vgl. Fittkau \& Maaß 2003), während die aktuelle EMNIDStudie nachweist, dass zwar noch immer mehr Männer online sind, aber ihr Anteil mit etwa 56\% deutlich gesunken ist. Auch hinsichtlich der Altersstruktur haben sich starke Veränderungen ergeben. Heute sind es bei weitem nicht mehr nur junge Menschen, die das Internet nutzen. Während der Anteil der Internet-Nutzer in der Altersgruppe der 14-19 jährigen bei 71,1\% liegt und hier sein Maximum erreicht, stehen die folgenden Altersgruppen der 20-29 jährigen $(68,6 \%)$ und der 30-39 jährigen $(59,1 \%)$ nur wenig zurück. Auch bei den $40-49$ jährigen ist noch fast jeder zweite online $(49,8 \%)$, erst mit weiter steigendem Alter nimmt die Nutzung des Internets drastisch ab: Bei den über 70 jährigen liegt der Anteil der Nutzer und derjenigen, die innerhalb eines Jahres online gehen wollen, bei nicht einmal $9 \%$, wobei sich in dieser Gruppe der Internetnichtnutzer überproportional viele Frauen befinden. Diese Geschlechts- und Altersstruktur verdeutlicht die Abb. 3 noch einmal - sie zeigt zudem, wie schwach die Gruppe der Intender ist und stützt somit auch die Vermutung, dass derzeit eine gewisse Marktsättigung eingetreten ist bzw. dass unter den Nichtnutzern erst neue Nutzerpotenziale erschlossen werden müssen. 

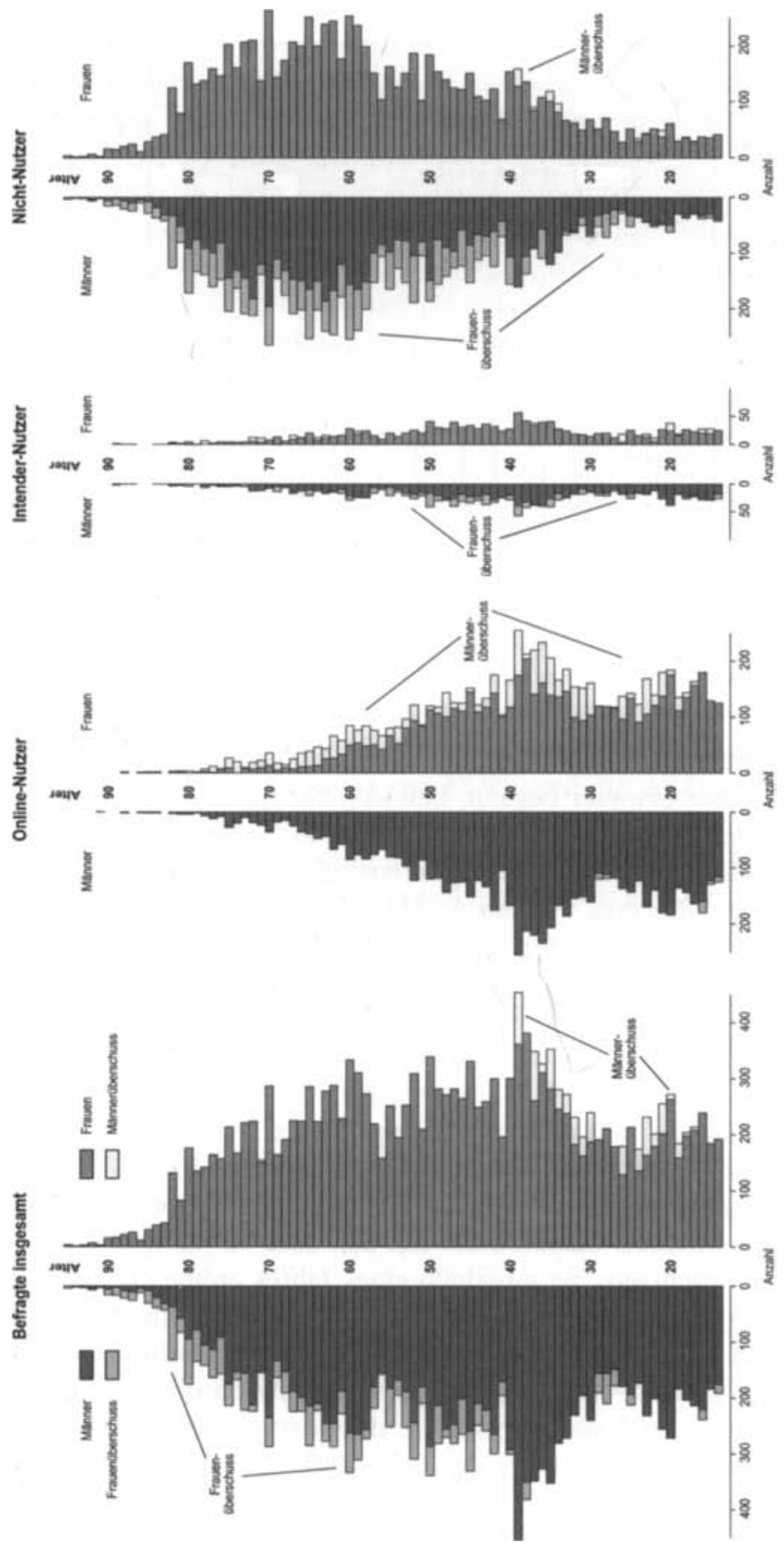

Abbildung 3: Onliner, Intender und Offliner nach Geschlecht und Alter (Quelle: EMNID 2002). 
Auch im Hinblick auf die Ausbildung der Internet-Nutzer hat sich die Basis deutlich erweitert: Nur noch ein gutes Viertel $(25,1 \%)$ der Onliner verfügt über Abitur oder ein abgeschlossenes Hochschulstudium, während das Gros einen Realschulabschluss $(37,6 \%)$ vorweisen kann. Personen mit Hauptschulabschluss stellen ebenfalls etwa ein Viertel der Onliner $(26,0 \%)$, während Schüler mit nur gut $10 \%$ die schwächste Gruppe unter den Internet-Nutzern ausmachen. Dies mag auf den relativ kleinen Anteil der Schüler an der Gesamtbevölkerung zurückzuführen sein, kann aber auch an den rechtlichen Vorschriften liegen, nach denen nur Personen über 14 Jahre in der Untersuchung berücksichtigt werden konnten.

Betrachtet man im Vergleich dazu die nach ihrer Ausbildung sortierten Gruppen hinsichtlich des Onliner- und Offliner-Anteils, so ergibt sich ein anderes Bild: Von allen befragten Schülern sind fast drei Viertel $(73,9 \%)$ online, was der größten Verbreitung unter allen Gruppen nach Bildungsabschlüssen entspricht. Ähnlich hohe Werte werden noch in der Gruppe der Personen mit Abitur (71,5\%) und derjenigen mit einem abgeschlossenen Studium erreicht, von denen noch zwei Drittel $(67,7 \%)$ das Internet nutzen. Den größten Anteil an Offlinern gibt es in der Gruppe der Hauptschulabsolventen, die keine Lehre absolviert haben: Hier nutzen $81,4 \%$ das Internet nicht und nur $6,6 \%$ planen, binnen eines Jahres das Internet zu nutzen:

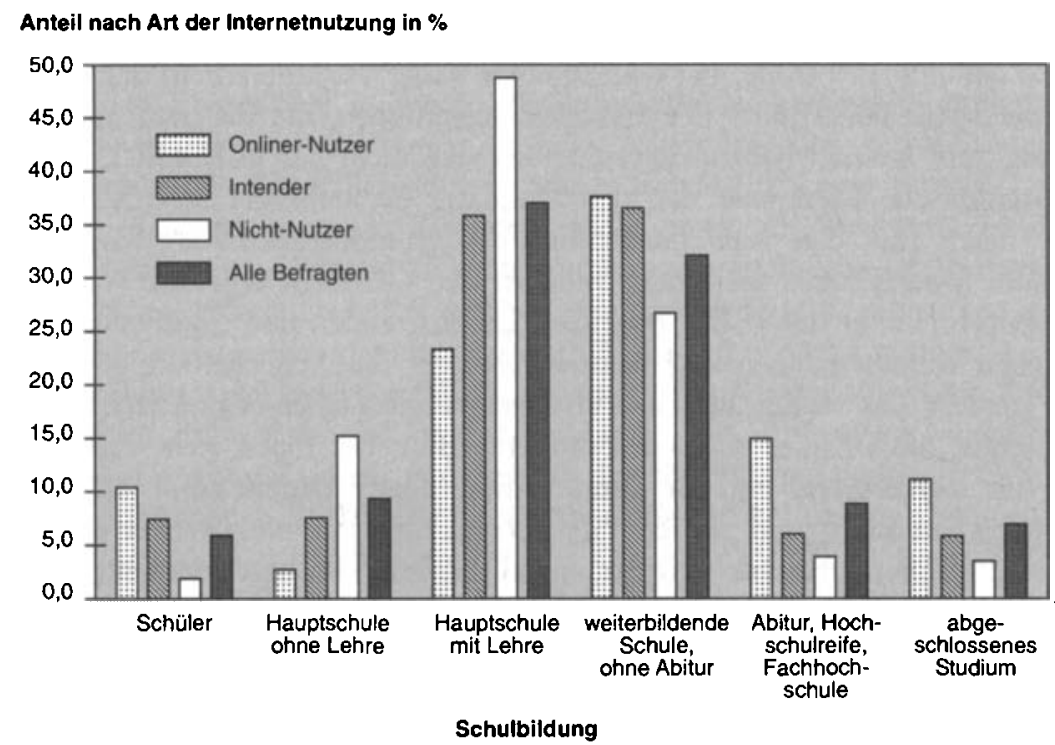

Abbildung 4: Internetnutzung nach Bildung (Quelle: eigene Abbildung nach EMNID 2002).

Allgemein kann also festgehalten werden, dass das Internet nicht mehr nur von ,jungen Studenten" genutzt wird. Vielmehr gibt es mittlerweile Nutzer mit allen Arten von Bildungsabschlüssen, jeden Alters und beiderlei Geschlechts. 
Nach wie vor steht den Nutzern aber eine fast gleich große Zahl an Nicht-Nutzern gegenüber. Diese Gruppe besteht vor allem aus älteren Menschen sowie Personen mit geringer Qualifikation. Beide Gruppen zeichnen sich zudem durch eine geringe Nutzungsabsicht aus, so dass nicht abzusehen ist, dass sich das Verhältnis zwischen Nutzern und Nichtnutzern sichtbar ändern wird - wahrscheinlich wird sich diese Angleichung erst mittelfristig ergeben, wenn die Nutzer, die das Internet bereits in ,jungen Jahren" kennen gelernt haben, in höhere Altersgruppen aufrücken.

Diese Veränderungen der Zusammensetzung der Internet-Nutzer könnten die Vermutung aufkommen lassen, dass sich auch der Zweck, zu dem das Internet genutzt wird, in den letzten Jahren geändert hat. Dem ist jedoch nicht so: 2001 nutzen die meisten Onliner das Netz im Zusammenhang mit ihrer beruflichen Tätigkeit oder, um Kontakte zu Freunden oder Familienmitgliedern herzustellen. Dazu werden vor allem e-Mails genutzt: ,e-mail represents over 85 percent of Internet usage, and most of this e-mail volume is related to work purposes, to specific tasks, and to keep in touch with family and friends in real life" (Castells 2001, S. 118). Das Internet ersetzt dabei keineswegs vorhandene Informationsund Kommunikationsmöglichkeiten, sondern wird als Ergänzung eingesetzt (vgl. Tracey 2001).

Dennoch werden weite Teile der Bevölkerung - vor allem Frauen und ältere Menschen - noch immer nicht über das Internet erreicht: So sind noch immer $56,3 \%$ der Frauen offline, bei den Senioren (über 70 Jahre) liegt der Anteil der Offliner sogar bei $91,6 \%$ ! Die Bevölkerungsgruppen, die bis dato noch keinen Zugang zum Internet haben, unterscheiden sich nicht nur in Hinblick auf soziodemographische Merkmale von den Onlinern. So stellt der „(N)Onliner Atlas

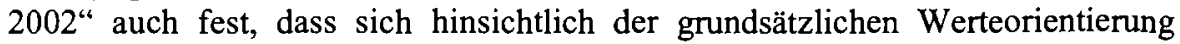
deutliche Unterschiede zwischen Onlinern und Offlinern ergeben. Während die Onliner vor allem durch die Begriffe "Lebensfreude“ und "Individualität" beschrieben werden können und deutlich weniger familienorientiert oder religiös sind, sondern statt dessen lust- und erlebnisorientiert leben (vgl. EMNID 2002, S. 20), zeigen die Offliner ein konservativeres Bild: Bei Ihnen steht Traditionelles mehr im Vordergnund und die Grundhaltung dieser Gruppe lässt sich über die Begriffe "Sozialität" und „Pflicht" definieren. So ist es nicht weiter erstaunlich, dass die Offliner auch eher familiär, sozial und religiös eingestellt sind (vgl. EMNID 2002, S. 40f.). Einen ähnlichen Unterschied stellte auch die ARD/ZDFOnline-Studie 2002 fest: Thr zufolge sind unter den Onlinern überdurchschnittlich viele ,junge Wilde", „Erlebnisorientierte" und „Leistungsorientierte“ zu finden, während die Offliner als „zurückgezogen" oder ,klassisch kulturorientiert" beschrieben werden (van Eimeren et al. 2002, S. 349). Diese unterschiedlichen Wertvorstellungen sind grundsätzlich auf die bereits diskutierten soziodemographischen Merkmale beider Gruppen zurückzuführen, in denen sich die je nach Zeitraum und Rahmenbedingungen unterschiedlichen Erziehungsmethoden und die von der Gesellschaft vermittelten Werte ausgewirkt haben. Die Nutzung des Internets spaltet die Bevölkerung demnach nicht nur hinsichtlich der Ausbildung oder der finanziellen Ausstattung, sondern offensichtlich auch nach ihren Wert- 
vorstellungen. Es wird damit klar, dass das Internet unterschiedliche Grundorientierungen der Bevölkerung deutlich voneinander trennt. Es ist kaum davon auszugehen, dass die gesamte Bevölkerung binnen kurzer Zeit zu den Onlinern zu zählen ist, da dies auch bedeuten würde, dass eine Anpassung oder eher eine Angleichung der Normen und Wertvorstellungen der Noch-Offliner erfolgen müsste.

Im nächsten Schritt soll nun die nach verschiedenen Regionen differenzierte Nutzung des Internets im Mittelpunkt des Interesses stehen.

\section{DIE INTERNET-NUTZUNG IN IHRER RÄUMLICHEN DIFFERENZIERUNG ${ }^{6}$}

Grundsätzlich ist ein Internet-Zugang überall dort möglich, wo auch ein Telefonanschluss verfügbar ist, wobei dies auch Mobilfunknetze mit einschließt. Dies bedeutet, dass in westlichen Ländem tatsächlich davon die Rede sein kann, dass der Internet-Zugang eine Ubiquität ist. Dennoch ist auch in diesem Zusammenhang Quantität nicht gleich Qualität. Im Zuge der Ausbreitung des Internets stieg auch das Volumen der übertragenen Daten sukzessive an. Dieser Anstieg ist auch auf die zunehmende Zahl der Nutzer zurückzuführen, hat seine Ursache aber vor allem in der gestiegenen Komplexität der übertragenen Informationen: Reichte in den Anfangstagen des Internets eine einfache HTML-Seite, um die gewünschte Information zu übermitteln, so werden die Informationen heute mit Grafiken, Bildern, Framesets sowie Programmen in Java, Javascript oder ActiveX (Applets) angereichert. Auch die in den letzten Jahren aufgekommenen Internet-Tauschbörsen (Napster, Kazaa u.a.), auf denen nicht mehr nur Musiktitel im MP3Format, sondern auch Software-CDs und mittlerweile auch Film-DVDs getauscht werden können, erfordern den Austausch sehr großer Datenmengen. Um diesen Austausch zu ermöglichen, ist eine leistungsfähige Infrastruktur nötig - und zwar sowohl im Bereich der Backbone-Netze als auch beim Netzanschluss des Endkunden. Sie führt dazu, dass der Internet-Anschluss mit Hilfe eines Modems und eines Telefonanschlusses mehr und mehr in den Hintergrund gedrängt wird. Dies liegt an der limitierten Bandbreite dieser Anschlussart, bei der die Daten in Tonsignale umgewandelt werden und die derzeit bei max. $56 \mathrm{Kbit} / \mathrm{s}$ liegt. Über dieselben Anschlusskabel (i.d.R. zweiadrige Kupferkabel) sind mittels DSL-Technologien Übertragungsgeschwindigkeiten von bis zu 2,3 Mbit/s (für Firmenkunden, für Privatkunden derzeit maximal 1,5 Mbit/s) möglich. Insbesondere in Hinblick

6 Im Allgemeinen ist es möglich, während der Internet-Nutzung den Standort des Nutzers grob zo lokalisieren. Hierzu werden Informationen der Network-Information Center verwendet, die Auskunft über die Zuordnung von IP-Adressen geben. Für Server und in Unternehmen werden diese Adressen, die mittlerweile ein knappes Gut sind, fest zugewiesen. Für andere Nutzer werden sie dynamisch zugewiesen, so dass eine genaue Lokalisierung kaum möglich ist. $\mathrm{Zu}$ mindest eine grobe Einordnung kann bei großen ISP vorgenommen werden. Entsprechende Verfahren werden z.B. bei mehrsprachigen Websites zur Wahl der Sprache oder für die Einblendung von landesspezifischer Werbung verwendet. 
auf künftige Telekommunikationsdienstleistungen (z.B. sog. „Echtzeitdienstleistungen“ wie Videokonferenzen oder Internettelefonate (,Voice over IP“; vgl. TRICK 2002, S. 15), wird der Ausbau der Leistungsfähigkeit der Netzinfrastruktur weiter vorangetrieben. Fraglich bleibt in diesem Zusammenhang jedoch, ob für die zahlreichen neu konzipierten Dienste auch eine entsprechende Nachfrage besteht, die den Fortbestand der Netze durch die Kundenakzeptanz dieser Dienste sicherstellen kann. So werden zwar bei TRICK (2002, S. 7) zahlreiche Anforderungen an eine künftige Kommunikationsinfrastruktur aufgelistet: Diese soll sowohl für Multimedia-Anwendungen geeignet sein als auch kostengünstige Anschlüsse gleicher Qualität in Stadt und Land bereit stellen. Derartige Forderungen setzen aber stillschweigend voraus, dass die Nachfrage in allen Teilräumen gleich stark ist, da sich ein gleichmäßiger Ausbau sonst nicht rechnen würde. Außerdem wird davon ausgegangen, dass die konzipierten Multimedia-Anwendungen auch von nahezu allen Nutzern gleichermaßen gebraucht resp. nachgefragt werden. Leider ist es jedoch nicht möglich, die Akzeptanz neuer Dienste bereits im Vorfeld annähernd korrekt zu bestimmen. Solche prognostischen Untersuchungen sind stets mit großen Fehlern behaftet und unterstützen Fehlentwicklungen eher, als sie sie verhindern. Bestenfalls kann man hier eine Rückbesinnung auf das raumordnerische Leitbild der „Gleichwertigkeit der Lebensbedingungen in allen Teilräumen" unterstellen, die den qualitativ hochwertigen Zugang zu Telekommunikationsdienstleistungen als Teil dieser Lebensbedingungen sieht.

Trotz dieser Kritik am ungebrochenen Ausbauwillen der Netzbetreiber wird die Zahl der bis dato verfügbaren Breitbandanschlüsse gerne als Maßzahl für die „Anschlussqualität“ einer Gesellschaft interpretiert. In Deutschland waren 2001 insgesamt 1.935.000 Breitbandanschlüsse verfügbar, was 2,36 Breitbandanschlüsse pro 100 Einwohner bedeutet. Damit liegt Deutschland in Hinblick auf die verfügbare Infrastruktur für den Internet-Zugang etwa im Mittel der OECD, in der durchschnittlich 2,98 Anschlüsse je 100 Einwohner verfügbar sind. Besonders verbreitet sind Breitbandzugänge in Schweden (5,33 je 100 Einwohner), Kanada $(8,88)$ und - weit voraus an der Spitze! - in Korea $(17,23)$ (vgl. Weiss 2003, S. 22). Die besonders hohe Verfügbarkeit an Breitbandzugängen in Korea ist bemerkenswert. Sie resultiert aus der hohen Bevölkerungsdichte; in Korea kann eine große Zahl an Nutzern ohne größere technische Schwierigkeiten erreicht werden. Mit Kanada und Schweden liegen dagegen zwei Länder in der Breitbandversorgung an der Spitze, die weniger dicht besiedelt sind. Dies wird auch von Kellerman bestätigt, der für die relativ dünn besiedelten Länder Skandinaviens angibt, dass zwischen 64,4\% (Schweden) und 54,4\% (Norwegen) der Bevölkerung „online" sind - Dänemark und Island liegen in der Mitte zwischen diesen beiden Werten (vgl. Kellerman 2002, S. 180f.). Unklar sind allerdings die Gründe für die außergewöhnlich hohe Akzeptanz der neuen Medien in diesen Ländern. Bei der Suche nach einer Erklärung muss berücksichtigt werden, dass Innovationen im Medien-Bereich in Skandinavien stets sehr gut angenommen worden sind. So war in der Vergangenheit der Anteil der Telefonanschlüsse überaus hoch, Mobiltelefone waren schnell weit verbreitet, ebenso Personal Computer (vgl. Kellerman 2002, S. 163-169). Möglicherweise ist die disperse Siedlungsstruktur in diesen 
Ländern (im Gegensatz zur dichten Besiedlung und hohen Bevölkerungsdichte in Mitteleuropa) für die besonders hohe Nachfrage nach Medien verantwortlich - bei Streusiedlungen sind persönliche Kontakte nur selten möglich, so dass die aufkommenden Telekommunikationsmittel von Anfang an die Chance boten, nicht nur über größere Entfernungen zu kommunizieren, sondern auch im Nahbereich Kontakte aufzubauen oder zu intensivieren. In die gleiche Richtung deutet, dass die Internet-Dienste zu einem hohen Anteil zur Kontaktpflege in der Familie oder im Freundeskreis (s.o.) verwendet werden, so dass die Kontaktaufnahme über das Internet insbesondere für Bewohner dünn besiedelter Gebiete eine deutliche Erleichterung darstellt. Möglich war die skizzierte Entwicklung jedoch nur durch die Subvention der ländlichen Räume durch höhere Tarife in den Stadtregionen und den forcierten Netzausbau durch staatliche Telefongesellschaften (Kellerman 2002, S. 173f.).

Doch wie sieht die Internetnutzung in Deutschland auf regionaler Ebene aus? Wie bereits erwähnt kann davon ausgegangen werden, dass etwa $40 \%$ der deutschen Bevölkerung online sind. Auf dieser Grundlage liegen von den insgesamt 40 deutschen Regierungsbezirken 15 unter dem Bundesdurchschnitt. Bei diesen Bezirken handelt es sich vor allem um ostdeutsche Regierungsbezirke, grenznahe Regionen (z.B. Trier) sowie um altindustrialisierte Räume (z.B. Teile des Ruhrgebiets, das Saarland). Die hochverdichteten Regionen Deutschlands - Hamburg, München (Regierungsbezirk Oberbayern), das Rhein-Main-Gebiet (Regierungsbezirk Darmstadt), Köln, Stuttgart, Hannover u.a. - finden sich hingegen unter den Regierungsbezirken mit einem überdurchschnittlich hohen Anteil an Onlinern (vgl. die Säulendiagramme in Abb. 5). Es ist also nicht nur so, dass in den Ballungsräumen das größere Potenzial zur Internet-Nutzung verortet ist, sondern es kann auch festgehalten werden, dass der relative Anteil der Internet-Nutzer dort größer ist. Ursache für die Konzentration der Internet-Nutzer in den Ballungsräumen ist die soziodemographische Struktur der Ballungsräume: Ebenso wie bei den Internet-Nutzern ist der Anteil der Personen, die Abitur oder sogar ein Hochschulstudium vorweisen können, in den Verdichtungsräumen deutlich höher als in anderen Regionen. Eine Ausnahme bilden in diesem Zusammenhang die ostdeutschen Regierungsbezirke. In sämtlichen verstädterten Regierungsbezirken der neuen Bundesländer (z.B. Dresden, Leipzig, Chemnitz) lässt sich ein überproportional hoher Anteil an Personen mit Hochschulabschluss feststellen, ohne dass die Internet-Nutzung in diesen Regierungsbezirken signifikant höher ausfallen würde. Die Gründe für diese Abweichung von dem sonst festzustellenden Zusammenhang zwischen hohem Bildungsabschluss und intensiver Internetnutzung sind bislang unbekannt - es lässt sich nur vermuten, dass sich Unterschiede in der finanziellen Ausstattung im Vergleich zu anderen Bundesländern bemerkbar machen.

Besonders interessant ist die gemeinsame Darstellung von Onlinern und der regionalen Verteilung des Domainbesitzes: 


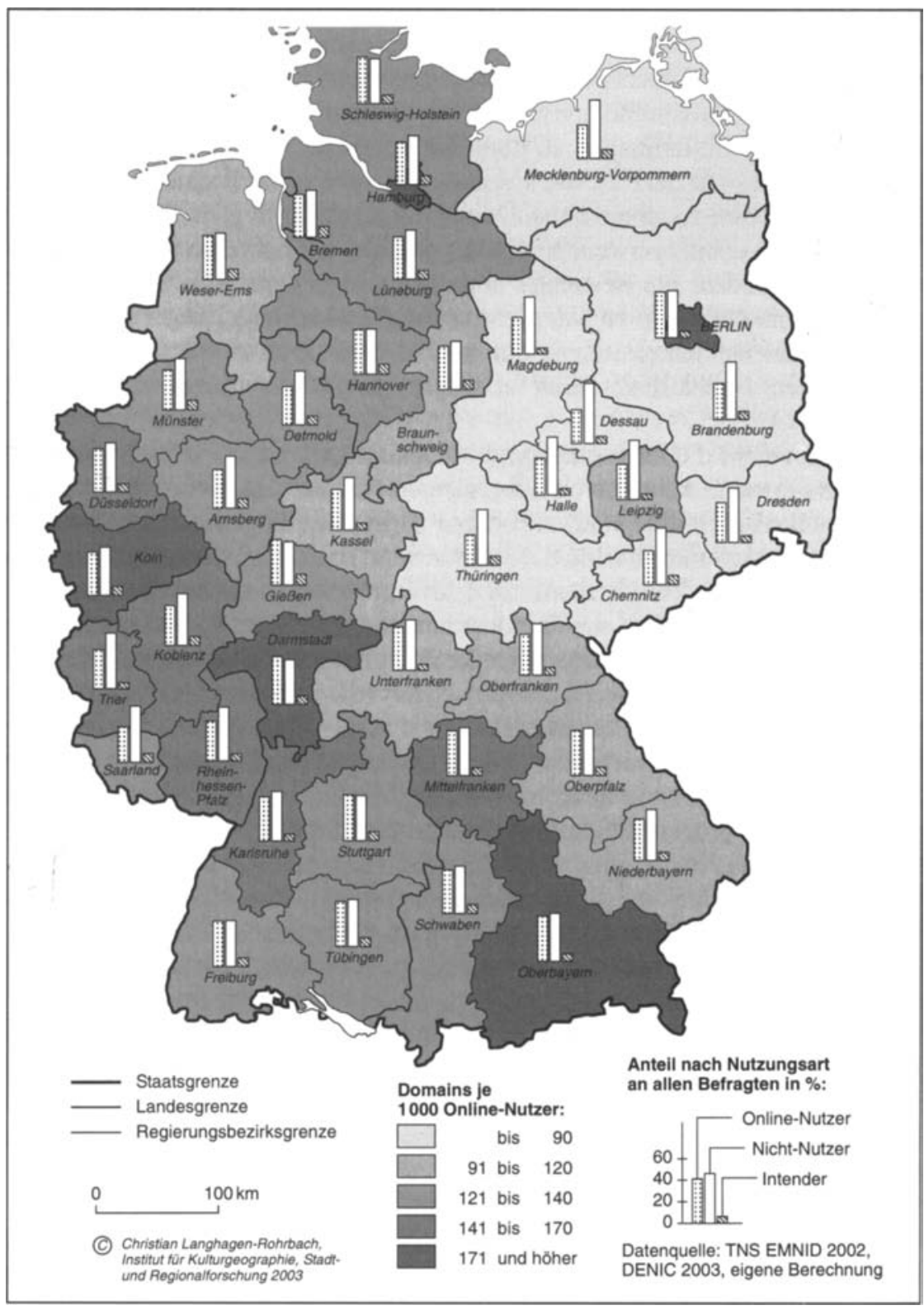

Abbildung 5: Regionalstruktur der Internetnutzung (Quelle: TNS EMNID 2002, DENIC 2003, eigene Berechnungen).

Sie zeigt deutlich, dass die Intensität der Nutzung des Internets im Westen Deutschlands deutlich höher ist als im Osten. Zur Zone intensivster Nutzung, also 
dort, wo sowohl ein hoher Anteil Onliner registriert wird als auch die Zahl der Domains je 1000 Onliner sehr hoch ist, gehört vor allem das Rheintal von Karlsruhe bis Düsseldorf inklusive der Region Rhein-Main, die Region von Oberbayern (München) über Ingolstadt nach Nürnberg sowie im Norden das Gebiet vom Ruhrgebiet ausgehend über Hamburg nach Schleswig-Holstein. Enklaven innerhalb des Ostens der Bundesrepublik bilden Berlin und der Regierungsbezirk Leipzig. Vergleicht man nun das Bild dieser Karte mit der Abb. 1, die die Struktur der Backbone-Netze in Europa zeigt, so fällt deutlich auf, dass innerhalb Deutschlands die höchste Nutzungsdichte dort zu finden ist, wo die meisten Netzwerkverbindungen zur Verfügung stehen. Dieser Eindruck verstärkt sich noch, wenn man die Verteilung privater City-Netze in Deutschland betrachtet, die sich ebenfalls in den genannten Teilen Deutschlands konzentrieren (vgl. Langhagen-Rohrbach 2001, S. 428). Offensichtlich besteht ein Zusammenhang zwischen der OnlineNutzung, dem Domain-Besitz und der Verfügbarkeit hervorragender Telekommunikationsinfrastruktur. Dabei ist es kaum möglich, Aussagen darüber zu machen, ob die hohe Nutzungsdichte in den beschriebenen Gebieten Ursache oder Folge der ausgezeichneten Infrastruktur ist, da anzunehmen ist, dass das eine das andere beeinflusst, so dass ein sich selbst verstärkender Prozess in Gang gesetzt wird.

Das vorliegende Nutzungsmuster stützt die eingangs von Castells (2001, S. 212) zitierte These, nach der urbane Regionen die höchste Nutzungsintensität aufweisen. Dabei zeigt sich jedoch auch, dass Deutschland die polyzentrische Struktur seines Städtesystems zugute kommt, da diese dazu führt, dass sich die Internet-Nutzung im Westen weniger stark auf einzelne Städte konzentriert, sonderm sichtbar verteilt ist (Castells 2001, S. 220). Der Osten Deutschlands fällt hier jedoch deutlich zurück, so dass einzelne Verdichtungsräume - Berlin sowie HalleLeipzig - mit relativ hohen Nutzungsintensitäten auffallen. Dies bedeutet, dass das Internet wenig dazu beiträgt, die Struktur des deutschen Siedlungssystems zu verändern, sondern eher dazu führt, dass sich die existierende Struktur weiter verfestigt.

Wie niedrig die Nutzung des Internet in den deutschen Großstädten tatsächlich ist, unterstreicht noch einmal die Abb. 6, in der der Anteil der Onliner, der Offliner und der Intender bezogen auf die Größe der Wohnsitzgemeinde dargestellt ist. Zwar lässt sich hier ablesen, dass es mehr Onliner gibt, je größer eine Gemeinde ist, aber bezogen auf alle deutschen Städte mit mehr als 500.000 Einwohner lässt sich sagen, dass selbst hier der Anteil der Offliner - wenn auch nur knapp - den der Onliner überwiegt. Je kleiner eine Gemeinde ist, desto kleiner ist auch der Anteil der Onliner. Der größte Anteil der Intender findet sich in Klein- und Mittelstädten mit 5.000 bis 20.000 Einwohnern. Sowohl in kleineren Gemeinden, als auch in größeren fällt das Interesse am Internet niedriger aus. In beiden Fällen kann der geringe Anteil derjenigen mit Nutzungsabsicht wie der große Anteil der Nichtnutzer strukturell begründet sein: Sowohl in kleinen Gemeinden in ländlichen Räumen, als auch in Großstädten leben zahlreiche ältere Menschen, die bislang nur wenig Interesse am Internet und seinen Möglichkeiten zeigen. In den Metropolen mag dieser Effekt noch durch einen vergleichsweise hohen Anteil 
Personen mit niedrigerer Qualifikation verstärkt werden, da diese das Internet bislang auch nur unterdurchschnittlich intensiv nutzen.

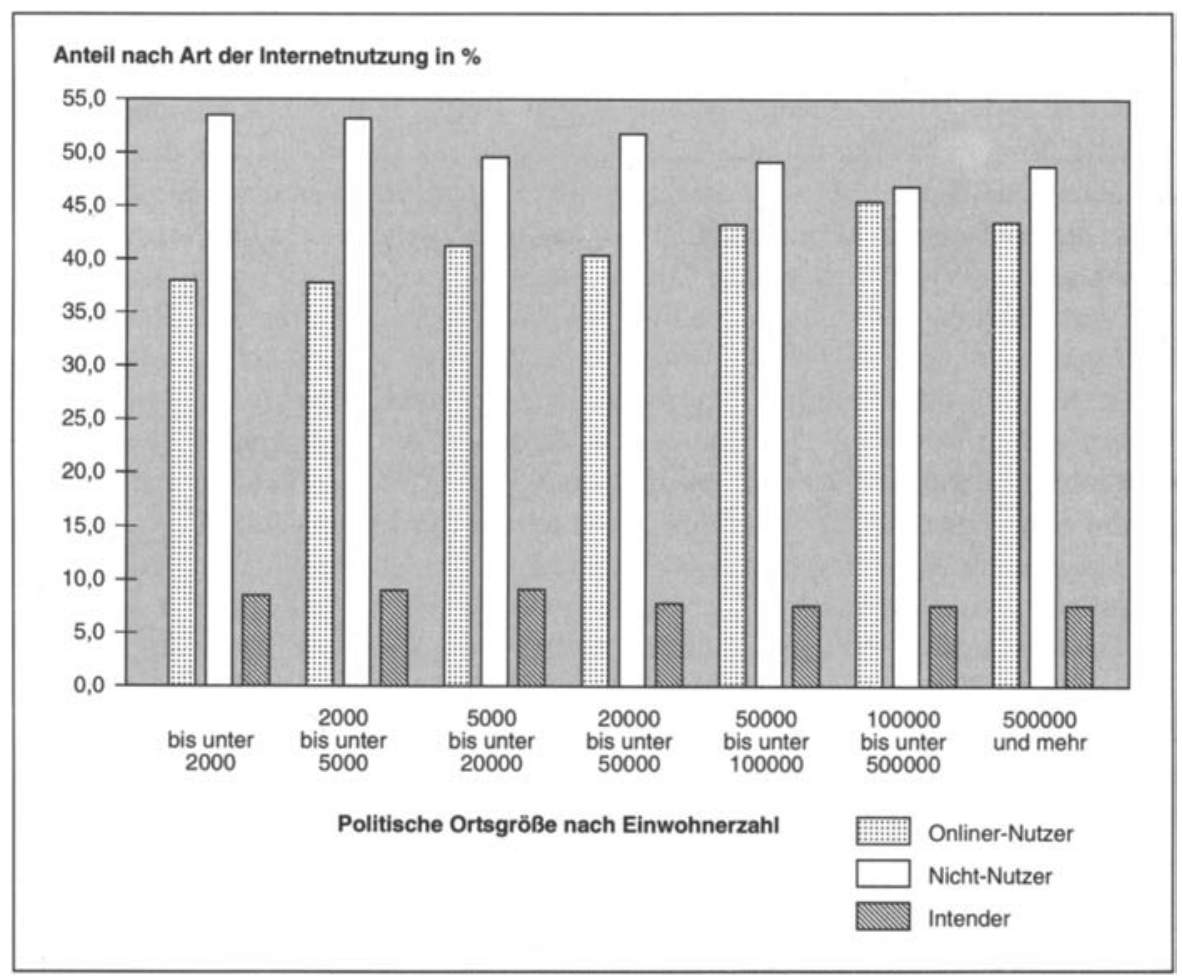

Abbildung 6: Onliner, Offliner und Intender in Städten und Gemeinden nach Einwohnerzahl (Quelle: eigene Abbildung nach EMNID 2002).

\section{INTERNET-NUTZUNG IN DEN STÄDTEN}

Wie soeben angedeutet, stellen die Städte als Knoten in den glokalen Datennetzen auch Orte mit besonders hoher Nutzungsdichte dar. Vor allem im Bereich der Domain-Namen können die Städte besonders hohe Konzentrationen verbuchen. So sind fast 30\% der Domains in Großbritannien in London registriert, in Spanien entfallen auf Madrid und Barcelona sogar insgesamt über 50\% (Castells 2001, S. 220). Auf Grund der polyzentrischen Struktur des deutschen Stäđtesystems ist die Konzentration hier nicht so groß (vgl. Langhagen-Rohrbach 2002).

Hinsichtlich der Nutzerstruktur lässt sich nach EMNID (2002) festhalten, dass es in Deutschland Städte gibt, in denen der Anteil der Nutzer besonders hoch ist. An der Spitze liegt Frankfurt am Main (53\% Onliner-Anteil), gefolgt von Dresden und Stuttgart (je 51\% Onliner-Anteil). Frankfurt am Main gilt seit längerem als 
„Internet-Hauptstadt“ Deutschlands, da hier auch mit „DE-CLX“ der bislang größte Internet-Knoten angesiedelt ist und die Stadt über eine ausgezeichnete Netzinfrastruktur verfügt. Auch der Finanzplatz könnte sich in dem hohen Anteil an Internet-Nutzern bemerkbar machen. Besonders erstaunlich ist, dass mit Dresden eine ostdeutsche Stadt auf dem zweiten Platz liegt, noch vor den eigentlich als Medienstädten bekannten Städten Köln und München (Rang 4 bzw. 5 mit 50\% und 49\% Onliner-Anteil). Möglicherweise ist dies auf verschiedene Faktoren wie die Technische Universität in Dresden und das in und um Dresden lokalisierte Hightech-Cluster der Chip-Industrie (AMD; Infineon) zurückzuführen. Da verschiedentlich - z.B. bei Mosco (2000) oder bei Castells (2001) - festgestellt wird, dass die Internet-Nutzung in „Technopoles“ besonders hoch ist, würde die in und um Dresden vorhandene Wirtschaftsstruktur diese These stützen.

Dennoch ist die Internet-Nutzung in deutschen Städten im Vergleich zu anderen Städten im Ausland gering: So erreicht das Internet in zahlreichen Städten der USA deutlich mehr als 60\% der Bevölkerung - im Jahr 2001 waren fast $70 \%$ der Haushalte von Portland, Seattle und San Francisco online (vgl. Kellerman 2002, S. 189). In Asien werden ähnlich hohe Anschlussraten erreicht (vgl. ebd., S. 191).

\section{SCHLUSSFOLGERUNGEN}

Die Darstellungen zur regionalen Nutzung des Internets haben sehr deutlich gezeigt, dass von einem „Ende der Geographie“ kaum die Rede sein kann - im Gegenteil. Zwischen der Nutzung des Internets und der vorhandenen Infrastruktur scheint es eine enge Verbindung zu geben. So führen Unterschiede in der Qualität der Internetversorgung auch zu räumlichen Nutzungsmustern, die regionale Entwicklungsunterschiede aus der Zeit vor dem Internet nachzeichnen. Eine besonders hohe Nutzungsintensität kann in unmittelbarer Nähe leistungsfähiger Datenleitungen und -knoten festgestellt werden, und zwar nicht nur in Deutschland, sondern weltweit (vgl. z.B. Castells 2001, S. 210-215 oder Shiode und Dodge 2000, S. 48-50). Das Internet trägt also nicht dazu bei, regionale Disparitäten abzubauen, sondern es verfestigt bereits bestehende Raummuster weiter. Das vielfach beschworene „Ende der Geographie“ bleibt somit aus, denn auch durch das Internet verlieren Orte nicht an Bedeutung, sondern sie lassen sich neu kategorisieren. Traten in der klassischen Hierarchie der Städte bestimmte Ausstattungsmerkmale in den Vordergrund, so ist heute die Bandbreite, mit der eine Stadt angebunden ist, ebenso entscheidend wie die Tatsache, ob sie innerhalb der globalen Netzwerke eine Knotenfunktion inne hat oder nicht. Demnach kann die Internet-Nutzung auch als ein Indikator für wirtschaftliche Prosperität und in der Folge auch für die Höhe der verfügbaren Einkommen verstanden werden. ${ }^{7}$ Diese Hypothese würde z.B. durch die im gesamtdeutschen Vergleich niedrigeren Einkommen und die gleichzeitig geringere Nutzerquote in den neuen Bundesländern

7 Vgl. zur wirtschaftlichen Indikatorfunktion der Internetnutzung und -verbreitung auch den Beitrag von Heinze in diesem Band, insb. Kap. 4.2. 
gestützt. Einen weiteren Hinweis auf die Richtigkeit dieser Hypothese könnte die oben beschriebene Sondersituation der Stadt Dresden bieten, in der infolge der dortigen Hightech-Industrie auch ein höheres Einkommensniveau zu erwarten ist.

An der vorhandenen Infrastruktur orientiert sich auch die Intensität der Internet-Nutzung. In den Verdichtungsräumen - also dort, wo die Infrastruktur besonders gut ausgebaut ist - konzentrieren sich sowohl die Besitzer von Domains als auch die Onliner in besonderem Maße. Ostdeutschland hingegen ist „digitales Hinterland“, in dem der Besatz mit Domains und die Internet-Nutzung nur unterdurchschnittlich sind. Allerdings darf man dabei nicht übersehen, dass Deutschland insgesamt im Vergleich $\mathrm{zu}$ anderen Nationen mit einem Onliner-Anteil von rund $40 \%$ auch nur einen Platz im Mittelfeld belegt - die Förderung der InternetNutzung ist also nach wie vor notwendig, vor allem, weil es den Anschein hat, als würde sich das Internet als eines der selbstverständlichen Kommunikationsmedien der Zukunft durchsetzen.

Dazu ist zunächst ein konsequenter Ausbau der Infrastruktur notwendig, um insbesondere die Versorgung größerer Bevölkerungsteile mit Breitbandzugängen sicher zu stellen. In Ostdeutschland verhindert der in den 1990er Jahren vorgenommene Ausbau der Kundenanschlüsse mit Glasfaserkabeln jetzt ein breites Angebot an DSL-Anschlüssen, da diese Technik auf normalen Kupferkabeln basiert. Durch Wireless LAN und UMTS könnten hier in naher Zukunft neue Zugangsmöglichkeiten zum Internet zur Verfügung stehen. Eine weitere Möglichkeit ist die Förderung öffentlicher Internet-Zugänge z.B. in Internet-Cafés. Eine Untersuchung der Stiftung Digitale Chancen (2002) hat gezeigt, dass auch bei diesen Einrichtungen deutliche Unterschiede zwischen verstädterten und ländlicher geprägten Regionen Deutschlands bestehen. Gerade in den Regionen der Bundesrepublik, in denen die Anteile der Offliner besonders hoch sind, sind weder eine adäquate Infrastruktur noch öffentliche Zugangsmöglichkeiten vorhanden. Es bleibt die Frage zu klären, wer für den Ausbau der technischen Infrastruktur verantwortlich ist. Durch die Deregulierung des Telekommunikationsmarktes tummeln sich zahlreiche private Anbieter auf diesem Markt, die aber vor allem darauf aus sind, hochwertige Leistungen für Unternehmen zu erbringen. Daher eignen sich für die Tätigkeit dieser Firmen vor allem Verdichtungsräume. Nach wie vor ist die Deutsche Telekom AG das einzige Unternehmen, das flächendeckend Zugangsmöglichkeiten für Telefon und Internet anbietet. Diese Versorgungsqualität in der Fläche kann ein privatwirtschaftliches Unternehmen kaum kostendeckend erbringen. Sollte die Deutsche Telekom AG vollständig privatisiert werden, wäre damit zu rechnen, dass die Versorgungsqualität in peripheren Region sinkt. Im Zweifelsfall könnte das bedeuten, dass in Gebieten, die für Unternehmen nur geringe Attraktivität besitzen, die Kommunen als Telekommunikationsanbieter für ihre eigene Einwohnerschaft auftreten müssten. Dies kann jedoch nicht im Interesse eines gesunden Telekommunikationsmarktes sein.

Flankierend zum Ausbau der Zugangsmöglichkeiten mit hohen Bandbreiten muss versucht werden, die bisherigen Nichtnutzer an das Internet heranzuführen. Kurse im Rahmen der Volkshochschulen, in Seniorenzentren etc. können hier die Neugierde dieser Personen wecken und diese in die Internet-Nutzung einführen. 
Dass auch bei diesen Bevölkerungsgruppen im ländlichen Raum Interesse an neuen Informations- und Kommunikationstechniken besteht, hat Fiedler nachgewiesen: So bekundeten $67,7 \%$ der 50 bis 65 jährigen und immerhin $40 \%$ der über $65 j a ̈ h r i g e n$, dass sie Interesse bzw. sehr großes Interesse an diesen Technologien hätten (vgl. Fiedler 2002, S. 45).

Insbesondere für Bevölkerungsgruppen, die im Berufsleben stehen, wird der selbstverständliche Umgang mit dem Internet und seinen Diensten immer mehr zum Berufsalltag gehören, da der Grad der Vernetzung in Deutschland aller Wahrscheinlichkeit nach weiter zunehmen wird - auf Dauer wird man der Nutzung des Internets kaum ausweichen können.

Für die Raumstruktur zeigt sich jedoch, dass das Internet und seine Nutzung keine wesentliche Veränderung mit sich bringen, sondern vorhandene Strukturen nutzen und verstärken. Wie oben beschrieben, werden die Möglichkeiten des Internets vor allem zur Kommunikation genutzt und zwar vor allem in der Familie und im Freundeskreis (vgl. Castells 2001, S. 118). Das eigentlich weltumspannende Netz und die These vom Ende der Geographie kehren sich somit um, da das globale Datennetz zur Verstärkung vorhandener regionaler und lokaler Siedlungsund Wirtschaftsstrukturen beiträgt. Außerdem nutzen Unternehmen die Datennetze zwar zur Kommunikation mit Niederlassungen, Zulieferern und anderen Unternehmen, aber insbesondere bei zentralen Unternehmensfunktionen zeigt sich, dass face-to-face-Kontakte nicht durch medial vermittelte ersetzt werden können. Trotz der weltumspannenden Netze führt dies dazu, dass Städte nach wie vor als wichtige Knotenpunkte Steuerungsfunktionen für Wirtschaft, Politik und Netze haben. Die Stadt zu Beginn des 21. Jahrhunderts präsentiert sich damit als „Ort“ der akkumulierten Informationsströme, wobei Zentralität vor allem gute Erreichbarkeit in medialen Netzen meint (vgl. Mitchell 1999, S. 155).

Den obigen Ausführungen zufolge nutzt das Internet vor allem gut ausgebildeten jungen Männern, wenngleich auch Frauen das Internet immer mehr für sich entdecken. Außerdem ist die globale Vernetzung über Datennetze für die Metropolen von Nutzen, da es ihre Funktionen und Bedeutung stärkt und nicht verringert. Dies bedeutet, dass sich auch das physisch-materielle Raumgefüge sowohl der Siedlungen als auch der Wirtschaft durch das Internet kaum ändern wird. Es bestätigt sich somit einmal mehr, dass die von Castells (1996) als ",space of flows“ bezeichnete Raumstruktur, in der einzelne „hubs“ dem Umschlag von Gütern, vor allem aber von Informationen, dienen, am ehesten in der Lage ist, die tatsächlich vorhandenen räumlichen Strukturen zu beschreiben. Damit ist auch klar, dass das Internet vor allem einem nutzt: Dem Bedeutungserhalt der Städte. 


\section{LITERATUR}

Caimcross, Frances (1997): The Death of Distance. How the Communications Revolution will change our lives, Harvard.

Castells, Manuel (1996): The Rise of the Network Society. The Information Age: Economy, Society and Culture, Vol. 1, Oxford (Massachusetts).

Castells, Manuel (2000): The Internet Galaxy. Reflections on the Internet, Business, and Society, Oxford.

Comcult Research (2002): Regionale Online-Nutzung. Nutzerbefragung auf meinestadt.de im Januar, online: http://www.comcult.de/ic/download/regionalenutzung2.pdf

DENIC (2003): Weiter großes West-Ost-Gefälle bei Domainzahlen, online: http://www.denic.de/doc/DENIC/presse/stats2002.html

Dodge, Martin und Rob Kitchin (2001): Atlas of Cyberspace, Harlow.

Van Eimeren, Birgit, Gerhard, Heinz und Brigitte Frees (2002): ARD/ZDF-Online-Studie 2002. Entwicklung der Onlinenutzung in Deutschland: Mehr Routine, weniger Entdeckerfreude, in: Media Perspektiven, Heft 8, Baden-Baden, S. 346-362.

EMNID (2002): (N)Onliner-Atlas 2002. Eine Topographie des digitalen Grabens durch Deutschland, Hamburg.

Fiedler, Christian (2002): Telematik im ländlichen Raum Bayerns. Möglichkeiten und Grenzen zur Minderung von Standortnachteilen in ländlichen Gebieten. Bamberger Geographische Schriften, Heft 21, Bamberg.

Fittkau und Maaß (2002): W3B-Ergebnisband. WWW-Benutzeranalyse Oktober/November 2002. W3B-Uni-Ergebnisband, Hamburg.

Kafka, G. (2001): Die Mär vom Breitbandzugang. Ländliche Regionen sind nur Zaungäste, in: DATACOM, Heft 1, S. 18-21.

Kellerman, A. (2002): The Internet on Earth: A Geography of Information, Chichester.

Krymalowski, Marc (2000): Die regionale Verteilung von Domainnamen in Deutschland (unveröffentlichte Diplomarbeit), Köln.

Langhagen-Rohrbach, Christian (2001): Internationale Datennetze und ihre Auswirkungen auf regionale Strukturen. Beispiele aus der Region Rhein-Main, in: Rhein-Mainische Forschung 119, Frankfurt a.M., S. 395-431.

Langhagen-Rohrbach, Christian (2002): Das Internet in Deutschland. Regionale Strukturen und Wachstumsmuster, das Beispiel der ".de-Domains", in: Raumforschung und Raumordnung, Heft 1, Köln, S. 37-47.

Mitchell, William (1999): E-Topia, Cambridge.

Mosco, Vincent (2000): Webs of Myth and Power, Connectivity and the New Computer Technopolis, in: Herman, Andrew und Thomas Swiss (Hrsg.): The World Wide Web and Contemporary Cultural Theory, New York, S. 37-60.

NUA.IE (2003): How many online? online: $\mathrm{http}: / / \mathrm{www} . n u a . c o m /$ surveys/how_many_online/europe.html

Perspektive Deutschland (2000): Projektbericht zur größten Online-Umfrage Deutschlands, online: http://www.perspektive-deutschland.de/files/ergebnisse/ProjektbroschuerePerspektive-Deutschland.pdf 
Shiode, Narushige und Martin Dodge (2000): Where on Earth is the Internet? An Empirical Investigation of the Geography of Internet Real Estate, in: Wheeler, James, Aoyama, Yuko und Barney Warf (Hrsg.): Cities in the Telecommunications Age. The Fracturing of Geographies, New York, S. 42-53.

Stiftung Digitale Chancen (2002): Gleiches Web für alle, in: FOCUS, Heft 13, S. 169.

Tracey, Karina (2001): Virtual Communities: What's New? (unpublished paper), Kansas.

Trick, U. (2002): Infrastruktur der Informationsgesellschaft. Weg zur ökonomisch effizienten, sozial gerechten und ökologisch verträglichen Fortentwicklung der Informations- und Kommunikationsgesellschaft,

online: http://www.e-technik.org/aufsaetze_vortraege/aufsaetze/trick_essay_infrastruktur. pdf

Weiss, Ernst (2003): Wie breit darf Breitband sein? Entwicklung von Breitband-Technologien hat hohes Zukunftspotential, in: DATACOM, Heft 1/2, S. 20-22.

Zook, Matthew (2002): The Geography of the Internet Industry: Venture Capital, Internet Startups, and Regional Development, Berkeley. 



\title{
INTERNET UND STADT \\ GEHT DEN KOMMUNEN DER ORT VERLOREN?
}

\author{
Holger Floeting
}

\begin{abstract}
Als Querschnittstechnologie greift das Internet in nahezu alle Bereiche des täglichen Lebens ein. Gerade Städte als Räume höchster Informationsdichte, Knoten der Netzinfrastruktur und Orte, an denen sich die Anwender konzentrieren, spüren dies. Bisher waren Städte und Gemeinden vor allem den Umgang mit ihren Bürgern gewohnt. Thr Handeln bezog sich auf einen klar abgrenzbaren Raum. Schon regionale Kooperationen über Verwaltungsgrenzen hinweg gestalteten sich oft mühsam. Durch das Internet werden klassische Definitionselemente der Stadt wie ihre administrativen Grenzen, räumlich kontinuierliche Strukturmerkmale, räumliche Distanzen oder die Einwohnergröße verändert und entwertet (vgl. Floeting/Grabow 1998, S. 17).

Die Folgewirkungen des Internets zeigen sich in der Kommune im Wesentlichen nicht als direkte, sondern als vermittelte Wirkungen, die nicht von der Technologie selbst, sondern von ihrer Anwendung in einem vielschichtigen sozialräumlichen Kontext bestimmt werden. Die weitgehende Durchdringung des kommunalen Alltags mit IuK-Technik fuihrt zur Bildung eines komplexen Geflechts von materiellem und virtuellem Raum (vgl. Floeting/Oertel 2002, S. 41). Räumliche Dimensionen verlieren dabei an Eindeutigkeit. Denn für die Nutzer des Internets ist es möglich, zugleich lokal und - per „Telepräsenz" - weltweit zu agieren. Auch die Auftritte der Städte und Gemeinden im Internet können nicht nur von der lokalen Bevölkerung, sondern weltweit genutzt werden. Mit der Präsenz im Internet zeigen sich die Städte daher einer weltweiten Öffentlichkeit, auch wenn sie sich nur ihren eigenen Bürgern präsentieren wollen. In noch stärkerem Maße als bisher vermischen sich dadurch lokale, nationale und globale Öffentlichkeiten.

Im Folgenden werde ich zunächst den Umgang der Kommunen mit neuen Technologien im Allgemeinen vorstellen, um im Anschluss daran den Entwicklungsverlauf der kommunalen Internetanwendungen nachzuzeichnen. Darauf aufbauend werden gegenwärtige strategische Ansätze und die Situation nach dem Ende des ersten Internetsbooms dargestellt, um abschließend zu der im Titel formulierten Frage Stellung zu beziehen.
\end{abstract}




\section{1. „AM ANFANG WAR DAS NETZ“ - KOMMUNEN UND IHR UMGANG MIT NEUEN TECHNOLOGIEN}

Seit dem Ende der 1970er Jahre haben sich Kommunen in Deutschland verstärkt mit dem Thema Informations- und Kommunikationstechnologien auseinandergesetzt. Stets standen drei Aspekte des Umgangs mit den neuen Technologien im Vordergrund:

- Infrastrukturaspekt: Kommunen waren immer wichtige Partner beim Ausbau der Informations- und Kommunikationsinfrastruktur, auch wenn ihr diesbezüglicher Handlungsspielraum nur begrenzt ist. Einerseits ging es dabei um den Ausbau der verwaltungsinternen Infrastruktur (z.B. Großrechner, Verwaltungsnetze, Intranet), andererseits um den Ausbau der Infrastruktur in den Städten und Gemeinden (z.B. Kabelfernsehen, Bildschirmtext, Stadtnetze, Mobilfunkstationen). Kommunales Engagement reichte von der Erteilung von Genehmigungen über die planerische Unterstützung des Ausbaus bis zur Beteiligung an Ausbaumaßnahmen oder dem selbständigen Ausbau.

- Nutzungsaspekt: Kommunen nutzen die neuen Informations- und Kommunikationstechnologien für die eigene Aufgabenwahrnehmung. Aus der technikunterstützten Informationsverarbeitung des Großrechnerzeitalters hat sich nach und nach das virtuelle Rathaus des Internetzeitalters entwickelt.

- Förderungsaspekt: Kommunen haben im Rahmen ihrer Aufgabenfelder auch immer den Einsatz neuer Technologien gefördert, z.B. im Rahmen der kommunalen Wirtschaftsförderung, der Stadtentwicklungspolitik, der Verkehrsentwicklung, der Bildungspolitik usw.

Zunächst beschäftigten sich die Kommunen mit der Ausstattung der eigenen Verwaltung mit EDV-Technik. Raumfüllende Großrechneranlagen, kommunale Rechenzentren, eine umfassende hierarchisch organisierte Infrastruktur und die Einführung von Bildschirmarbeitsplätzen waren die Kennzeichen dieser Phase des kommunalen Umgangs mit neuen Informations- und Kommunikationstechnologien. Es ging im Wesentlichen um die Reorganisation der internen Arbeitsabläufe unter Einsatz der neuen IuK-Technologie. Schon bald geriet aber auch die Schnittstelle zum Bürger in den Blick. Zu Beginn der 1980er Jahre wurde beispielsweise im Rahmen der Kabelpilotprojekte der Aufbau von Bürgerfernsehen und interaktiven Diensten diskutiert. Tatsächlich entstand eine umfassende Breitbandkabelinfrastruktur, von der vor allem die großen Verdichtungsräume profitierten. Der eine oder andere „Offene Kanal“ ging auf Sendung, erreichte allerdings meist nur eine kleine Zuschauer- oder Zuhörergemeinde. Die Entwicklung interaktiver Angebote scheiterte vor allem daran, dass ein Rückkanal (vom Empfänger zum Sender) fehlte. 
Selbst damals neue Technologien wie der Bildschirmtext, später Datex-J, dann T-Online ${ }^{1}$ konnten die erhoffte Interaktivität $z$ wischen Stadtverwaltungen und Bürgern nicht im gewünschten Umfang herstellen. Zwar waren in Boomzeiten etwa 150 Kommunen in Deutschland im Bildschirmtext mit Angeboten vertreten, doch fehlte es an Nutzern (vgl. Kubicek 1996, S. 78). Analysten hatten für 1990 zwischen 3 und 3,5 Millionen Bildschirmtextnutzer vorausgesagt. Tatsächlich nutzte zu dieser Zeit aber nur ein Zehntel davon die neuen Angebote. Die interaktive Kommunikation via Bildschirmtext war, verglichen mit den heutigen Möglichkeiten, eher kryptisch als intuitiv. Die Enttäuschung über die erzielten Effekte des Bildschirmtextes für die kommunale Arbeit, die im starken Kontrast zu manch vollmundigen Versprechen hinsichtlich seiner Potenziale standen, wirkte in vielen Kommunen noch einige Zeit nach. So entwich nicht wenigen kommunalen Akteuren in den ersten Diskussionen darüber, ob sich die Städte und Gemeinden nicht mit eigenen Angeboten im Internet engagieren sollten, der Stoßseufzer: „Nicht noch ein Bildschirmtext!"“

Insgesamt beschäftigte man sich in Deutschland - anders als in anderen Ländern - zunächst bei der Einführung neuer Informations- und Kommunikationstechnologien - seien es Großrechnernetze, Breitbandkabel, Glasfasertelekommunikationsnetze, ISDN, DSL oder Mobilfunknetze - mit einer weitgehenden Perfektionierung der Infrastruktur. Dies war auch in den deutschen Städten und Gemeinden nicht anders. Die ,kreativen“ Veränderungen von Rahmenbedingungen, die Entwicklung interessanter Anwendungen und Inhalte, der spielerische Umgang mit der Technologie und dessen Unterstützung etwa durch Experimentierklauseln waren dagegen in Deutschland bisher nicht so weit verbreitet. Deshalb kam es auch immer wieder zu dem Phänomen, dass nicht neue Technologien zur Lösung spezifischer Problemlagen entwickelt wurden, sondern neue „perfekte Technologien“ ihre Anwendungen und Anwender ,suchten“.

\section{2. „BIN ICH DRIN?““ - VON DER ERSTEN NETZPRÄSENZ ZUM UMFASSENDEN PORTAL}

Auch in den 1990er Jahren war der Umgang der Kommunen mit den Informations- und Kommunikationstechnologien zunächst einmal durch das Thema Infrastrukturausbau gekennzeichnet. Die Liberalisierung des deutschen Telekommunikationsmarktes wurde in einer Reihe von Kommunen dazu genutzt, lokale Telekommunikationsgesellschaften zu fördern. Etwa zur gleichen Zeit begannen die ersten Aktivitäten von Städten und Gemeinden im Internet, die sich in der Folge schnell entwickelten. Waren Mitte der 1990er Jahre noch mehr als zwei Drittel der deutschen Großstädte ohne elektronische Adresse (Floeting/Gaevert 1997, S. 4), haben heute selbst kleine Gemeinden ihr „Abbild“ im Internet. Anfangs ging es den Städten primär um die Präsenz im neuen Medium. Vorreiter waren vor allem die größeren Städte. Schon 1997 sahen $86 \%$ der deutschen Städte in der

1 Nicht zu verwechseln mit dem Internet-Angebot, das heute unter gleichem Namen existiert. 
Bereitstellung von elektronischen Stadtinformationen auch eine kommunale Aufgabe (Floeting 2001, S. 110).

Die ersten Angebote gingen häufig auf Aktivitäten einzelner Akteure in oder außerhalb der Stadtverwaltungen zurück. Wesentliche Impulse gingen von den Universitäten aus. Sie verfügten schon zu einem frühen Zeitpunkt nicht nur über eine vergleichsweise leistungsfähige Internetinfrastruktur, sondern auch über das notwendige kreative Milieu für die Entwicklung neuer Technologien und Informationsangebote. Außerdem stellten sie mit ihren Hochschulmitarbeitern und Studierenden auch die Hauptnutzergruppen der neuen Technologie. Während dem Normalbürger und vielen Verantwortlichen in den Städten die Bedeutung des Internets erst deutlich wurde, als auch die Tagesschau im Fernsehen eine wwwAdresse einblendete, gab es im Umfeld der Hochschulen schon frühzeitig das Bewusstsein für die Veränderungskraft der neuen Technologie. In manchen Hochschulen entwickelte sich bald die Einsicht, dass man für eine weitergehende Verbreitung des neuen Mediums Städte und Gemeinden als Partner brauchte. Ein Vorreiter dieser Entwicklung war Bremen, das noch heute eine führende Stellung unter den deutschen Städten bei der Weiterentwicklung der kommunalen InternetAngebote einnimmt.

Seit der Pionierphase haben sich die Internet-Auftritte von Städten und Gemeinden grundlegend gewandelt. Anfangs handelte es sich häufig um Imagebroschüren und Prospekte, die mehr oder weniger unverändert in Webseiten gegossen wurden. Grundsätzliche Stärken des neuen Mediums wie die Individualisierbarkeit von Angeboten, die mögliche Interaktivität oder die regelmäßige Aktualisierung und damit die Aktualität der Informationen spielten bei der ersten Gestaltung häufig noch keine Rolle. Es ging darum, im Internet „dabei“ zu sein. Aus diesen frühen Ansätzen entwickelten sich aber schnell umfassendere Stadtinformationssysteme mit statistischen Informationen und ersten Anteilen interaktiver Elemente. Die Angebote wurden kontinuierlich verbessert. Die hohe Innovationsgeschwindigkeit lag einerseits im starken Engagement einzelner Akteure begründet. Andererseits gab es kaum inhaltliche und organisatorische Regelungen, die Innovationen hätten behindern können. Die Internetangebote der Städte und Gemeinden waren im positiven Sinne „Experimentierfelder" für einen neuen Umgang der Kommunen mit ihren Bürgern und der Öffentlichkeit. In kurzer Zeit wurden die Angebote in den „Vorreiterstädten“ um Kommunikationselemente angereichert (in erster Linie um die Möglichkeit, über E-Mail zu kommunizieren). „Medienbrüche" in der Interaktion zwischen der Stadt und ihren Bürgern sind jedoch bis heute die Regel geblieben.

Mit der Konzentration der kommunalen Internet-Anstrengungen auf das Thema "Electronic-Government" wandte man sich dann besonders der Frage der medienbruchfreien Interaktion zu. Ziel war es, informationstechnisch unterstützte Großverfahren der Verwaltungen mit Schnittstellen zum Bürger zu versehen. Ergänzt durch Angebote für E-Commerce und E-Community (Kommunikation zwischen und innerhalb einzelner Akteursgruppen) entstanden schließlich umfassende städtische „Portale“. 
Insgesamt haben die Internet-Angebote der Städte und Gemeinden auf diese Weise erheblich an Interaktivität gewonnen. Verfügten 1997 nicht einmal ein Drittel der Angebote über die Möglichkeit der E-Mail-Kommunikation, so waren es im Jahr 2000 bereits rund 96\%. Zukünftig wird es wohl kaum ein Angebot geben, das nicht auch über diese Möglichkeit verfügt. Konnte man 1997 in nicht einmal einem Zehntel der Städte, die überhaupt im Internet vertreten waren, in Datenbanken recherchieren, so gab es diese Möglichkeit drei Jahre später bereits in mehr als der Hälfte der Internet-Angebote. Einige interaktive Angebote haben sich seit 1997 deutlich verbessert, haben aber ihren „Durchbruch“ immer noch nicht erzielt. So konnte man beispielsweise 1997 in weniger als 5\% der kommunalen Internetangebote direkte Buchungen vornehmen. Trotz erheblicher Fortschritte war dies aber auch im Jahr 2000 erst in weniger als einem Drittel der Angebote möglich.

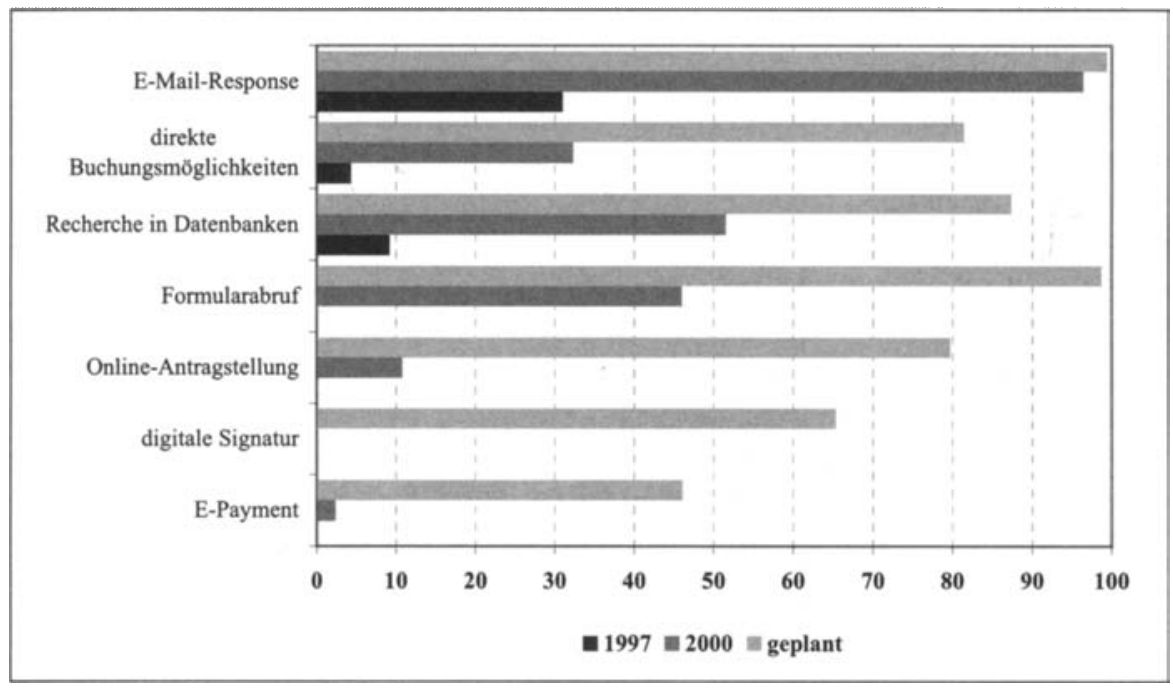

Abbildung 1: Anteil interaktiver Elemente in Online-Angeboten deutscher Städte (in \% der Städte) (Quelle: Floeting 2001).

An manche Möglichkeiten der interaktiven Nutzung von Internet-Angeboten wurde 1997 noch gar nicht gedacht; so etwa an den Formularabruf, der im Jahr 2000 dann bereits in $46 \%$ der Städte möglich war. Auch eine weitergehende Abwicklung von Verwaltungskontakten über das Internet, die Online-Antragstellung, die 1997 kaum vorstellbar war, war drei Jahre später bereits in mehr als $10 \%$ der Städte realisiert. Andere Angebote entwickeln sich weiter eher zögerlich; so etwa die E-Payment-Möglichkeiten oder der Einsatz von elektronischen Signaturen zur rechtssicheren Abwicklung von Transaktionen. Noch im Jahr 2000 wurden in keiner deutschen Stadt elektronische Signaturen eingesetzt, mittlerweile bieten immerhin 10 Städte diese Möglichkeit. Insgesamt zeichnet sich aber eine generel- 
le Entwicklung zu immer größeren Anteilen von Kommunikations- und Transaktionsangeboten ab (vgl. Floeting 2001, S. 110).

Dennoch wird auch bei der Entwicklung des E-Government wieder deutlich, dass man sich häufig auf die Perfektionierung der Infrastruktur und des Regelungsrahmens beschränkt und dabei die Entwicklung von für den Bürger bedeutsamen Inhalten und Anwendungen vernachlässigt. Zwar gab es in Deutschland schon vor der europaweiten Regelung ein Signaturgesetz, das den Einsatz digitaler Signaturen regeln sollte. Technisch einwandfrei funktionierende und die gesetzlichen Vorgaben erfüllende Signaturen gab es aber noch nicht. Auch lassen sich bis heute nur wenige Bürger davon überzeugen, Geld dafür auszugeben, eine solche Signaturmöglichkeit zu erhalten. Viele rechtliche und technische Anpassungsschritte sind noch nötig, um aus der ,perfekten“ Regelung funktionstüchtige Bürgerdienstleistungen für den Alltag zu machen.

Neben den virtuellen Rathäusern haben sich in den letzten Jahren auch virtuelle Marktplätze auf lokaler und regionaler Ebene entwickelt:

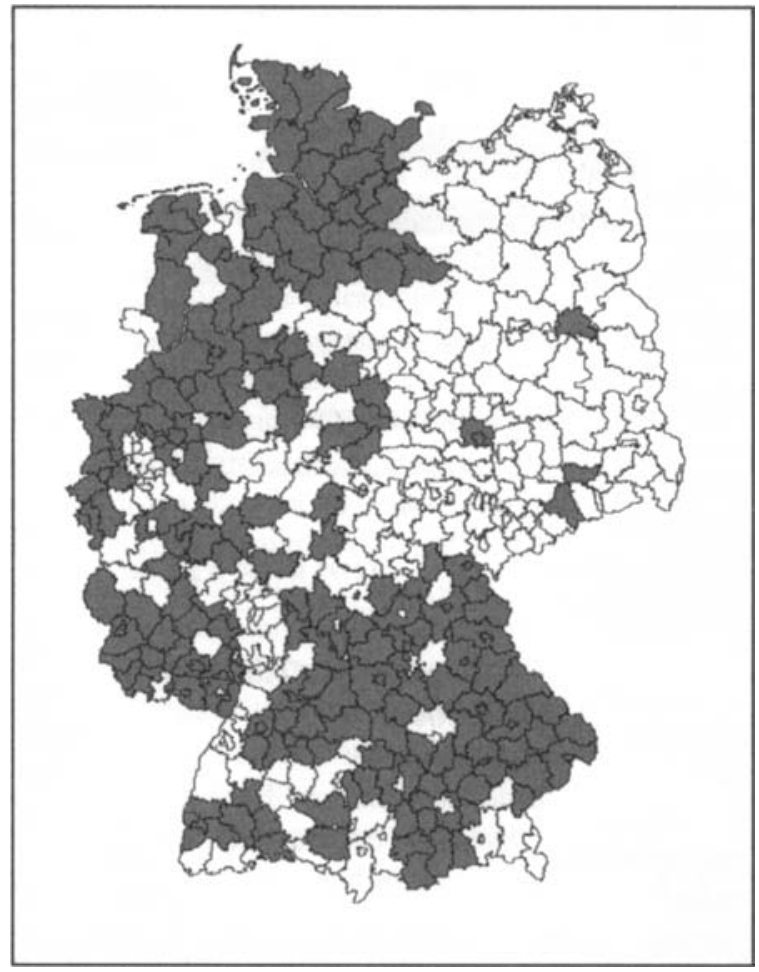

Abbildung 2: Lokale und regionale virtuelle Marktplätze (dargestellt sind nur Marktplätze in Kooperation mit Sparkassen; Quelle: eigene Erhebungen 2002: Kartographie: Seidel-Schulze). 
Mittlerweile gibt es vermutlich mehrere hundert solcher Angebote. Rund 100 lokale und regionale virtuelle Marktplätze sind allein unter der Beteiligung von Sparkassen $^{2}$ entstanden. Die genaue Zahl der virtuellen Marktplätze ist kaum zu ermitteln; zu viele Akteure betreiben Angebote, die sie selbst als virtuellen Marktplatz bezeichnen, nicht alle können aber den damit erhobenen Anspruch auch einlösen. Häufig finden sich nur mehr oder weniger gut gemachte Branchenverzeichnisse hinter der Bezeichnung ,virtueller Marktplatz“. Doppelangebote in bestimmten Regionen erschweren die Ermittlung der Anzahl zusätzlich.

\section{3. „JA, MACH NUR EINEN PLAN“ - VOM EXPERIMENTIERFELD ZUM STRATEGISCHEN ANSATZ}

Insgesamt hat sich der Umgang der Kommunen mit den Themen Informationsund Kommunikationstechnologien, „Neue Medien“ und Internet erheblich gewandelt. Aus dem „Experimentierfeld“ für einzelne engagierte Akteure und einige Vorreiterstädte hat sich ein umfassendes Handlungsfeld für Kommunen entwickelt. $40 \%$ der deutschen Städte (mit mehr als 50.000 Einwohnern) haben mittlerweile ein Strategiekonzept für die Realisierung eines "virtuellen Rathauses“ erarbeitet. Vor allem die großen Städte sind dabei schon weit vorangeschritten:

Dennoch ist erkennbar, dass mittlerweile weite Teile Deutschlands durch solche virtuellen Marktplätze abgedeckt werden. Ebenso offensichtlich ist die diesbezügliche Schwäche Ostdeutschlands. In manchen Bundesländern wurden - mit mehr oder minder großem Erfolg - Initiativen auf Landesebene gestartet, um virtuelle Marktplätze zu entwickeln. Mit Ausnahme dieser „von oben“ verordneten Kooperationen arbeiten aber nur wenige Marktplätze auf interkommunaler oder interregionaler Ebene zusammen. Anders als Angebote für Geschäftskunden (B2BMarktplätze) sind bisher nur wenige lokale und regionale virtuelle Marktplätze für Endkunden wirklich erfolgreich.

2 Neben den Kommunen, Verlagen und Telekom-Carriern gehören die Sparkassen zu den Hauptbetreibern solcher Angebote. 


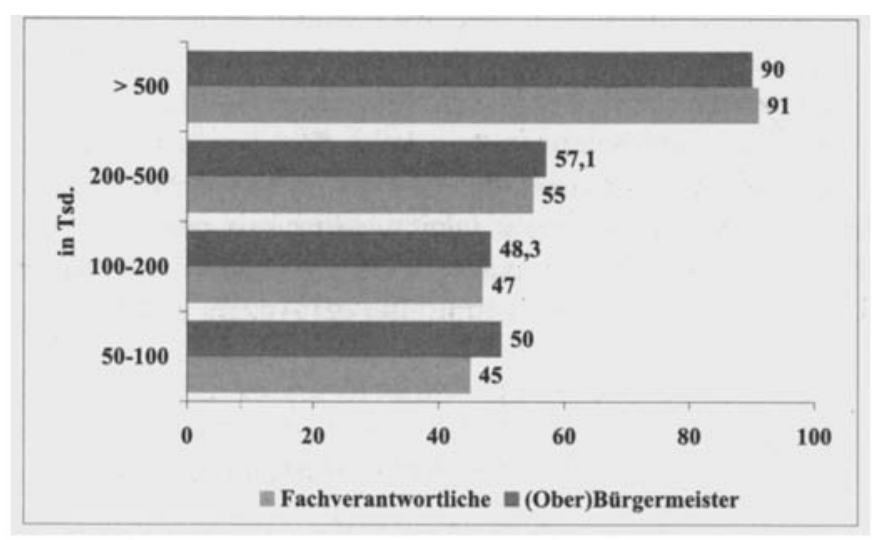

Abbildung 3: Existierende Strategiekonzepte der deutschen Städte für IuK, „Neue Medien" und Internet nach Einwohner-Größenklassen und nach Aussagen der Fachverantwortlichen und der Oberbürgermeister (Quelle: Difu-Umfrage ,Virtuelle Rathäuser und virtuelle Marktplätze in Stadt und Region 2000/2001“).

Bleibt man im Bild des virtuellen Rathauses, dann hatten 14\% der deutschen Städte mit 50.000 und mehr Einwohnern Anfang 2001 gerade die Pläne gezeichnet, rund $17 \%$ hatten den Grundstein für das virtuelle Rathaus bereits gelegt. Die meisten Städte waren gerade im Begriff das Fundament fertig zustellen. Erste Stockwerke gab es immerhin schon in knapp einem Fünftel der Städte und 5\% hatten sogar schon das Richtfest gefeiert. In rund $2 \%$ der Städte und Gemeinden gab es aber auch weiterhin keinerlei Pläne (vgl. Abb. 4).

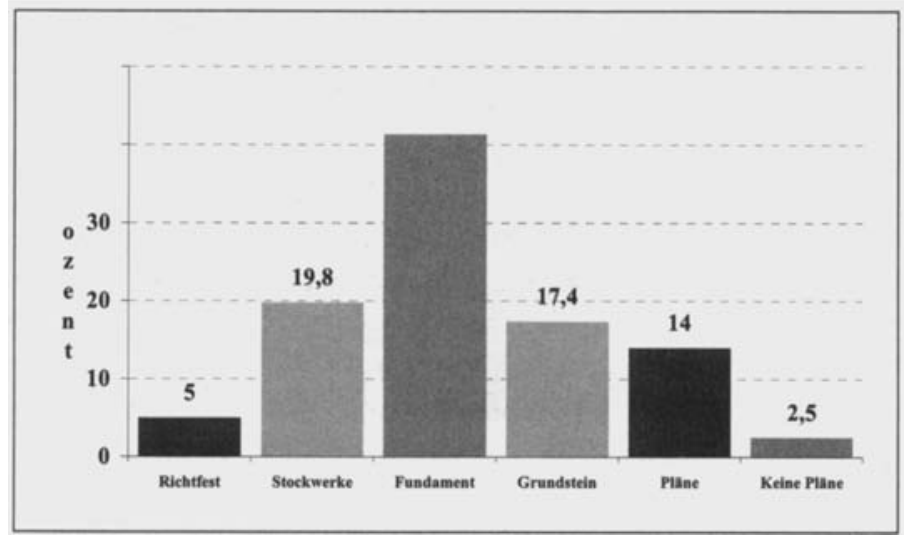

Abbildung 4: Entwicklungsstand des virtuellen Rathauses Anfang 2001 nach Einschätzungen der Fachverantwortlichen (Quelle: Difu-Umfrage ,Virtuelle Rathäuser und virtuelle Marktplätze in Stadt und Region 2000/2001"). 
Trotz des unterschiedlichen Entwicklungsstandes hat sich gegenüber den frühen 1990er Jahren die Bewertung der neuen Informations- und Kommunikationstechnologien durch die Kommunen stark verändert. Den Kommunen geht es heutzutage vor allem um die Förderung von Transparenz und Bürgerbeteiligung, die Optimierung des Verwaltungshandelns, die Verbesserung der internen Kommunikationsfähigkeit und, damit verbunden, um die Steigerung der Mitarbeitermotivation sowie um die positive Darstellung der Stadt im Rahmen des Standortmarketings. Die technologischen Veränderungen werden als Chance einer umfassenden Modernisierung gesehen, was sich in der Diskussion um das Thema „Electronic Government“ zeigt. In einigen Städten wird mit E-Government nicht nur die Einführung einer neuen, auf Informations- und Kommunikationstechnologie gestützten Anwendung im Verwaltungsbereich, sondern eine umfassende Modernisierungsstrategie des öffentlichen Sektors bezeichnet. E-Government umfasst dann ,alle Aspekte des Regierens und Verwaltens (öffentliche Willensbildung, Entscheidungsfindung, Leistungserstellung und -erbringung, Partizipation), sofern sie durch die Nutzung von Informations- und Kommunikationstechnologien unterstützt und verbessert werden können" (Difu 2002, S. 6).

\section{4. „CRISIS? WHAT CRISIS?“ - KOMMUNEN NACH DEM ENDE DES ERSTEN INTERNET-BOOMS}

Auch der kommunale Umgang mit neuen Informations- und Kommunikationstechnologien scheint im Zeitverlauf deutlichen Schwankungen zu unterliegen. So folgten Phasen der Aufmerksamkeits-Hochkonjunktur bisher stets Phasen der Desillusionierung bzw. der Konsolidierung.

Die Desillusionierungen hängen u.a. mit den häufig euphorischen Versprechen zusammen, die mit technologischen Innovationen verbunden werden. Versprochen wurde etwa, dass die neuen Informations- und Kommunikationstechnologien einen "Quantensprung in der Lebensqualität" (so der damalige EU-Kommissionspräsident Jacques Santer, zit. n. German 1996, S. 16) mit sich bringen würden. Informations- und Kommunikationstechnologien wurden als universelle Problemlöser für nahezu alle drückenden gesellschaftlichen, wirtschaftlichen und ökologischen Fragestellungen dargestellt. 4 Gerade die Promotoren der neuen Technologien beschränkten sich häufig auf die Schilderung der technologischen Potenziale, ohne zu bedenken, dass die technologischen Potenziale erst ausgereizt werden können, wenn auch die dafür notwendigen organisatorischen Voraus-

4 „Ohne jeden Zweifel bieten die neuen Informations- und Kommunikationstechnologien ungeheure Chancen in vielfacher Hinsicht: neues Wachstum und die Schaffung von Arbeitsplätzen, eine effizientere Nutzung der Inputs, d.h. nicht nur der Arbeit, sondern auch der Energiequellen, der Rohstoffe und des Kapitals, was zu einer umweltgerechteren Entwicklung beitragen könnte; höhere Einkommen und ein Zugewinn an Wohlfahrt; dezentrale Organisationsformen sowohl in den Betrieben als auch im Sinne der Erwerbsbeteiligung, planmäßigerer Verlauf der regionalen und städtischen Entwicklung, eine Bereicherung des persönlichen Lebens sowie eine demokratischere Entscheidungsfindung" (Gruppe hochrangiger Experten ..., HLEG 1996, S. 49). 
setzungen und Verhaltensänderungen der Akteure stattgefunden haben. So wiederholen sich die Versprechen, die mit der Einführung neuer Informations- und Kommunikationstechnologien verbunden werden, stets aufs Neue. Schon bei der Planung des Bildschirmtextes Ende der 1970er Jahre versprach die Deutsche Bundespost als zukünftiger technischer Betreiber, dass über das neue Medium Behördeninformationen wie Besuchszeiten, Sitzungstermine von Parlamenten oder lokale Verordnungen bereitgestellt werden könnten. Anfang der 1980er Jahre empfahl der Deutsche Städtetag seinen Mitgliedern das Engagement im Bereich der Breitbandkabelnetze, denn über diese Infrastruktur könne man Sozial-, Familien-, Jugend- und Seniorenberatung ebenso anbieten wie das Ausfüllen von Formularen oder die Überwachung von Kinderspielplätzen durch die Polizei. Der Blick zurück macht deutlich, dass viele der informationstechnischen Wohltaten, die heute unter der neuen Überschrift „Electronic Government" für die Entwicklung des städtischen Lebens, die Partizipationssteigerung der Bürger und die Bürgernähe der Verwaltung versprochen werden, schon seit einem Vierteljahrhundert zu den üblichen Verkaufsargumenten für die jeweiligen neuen Technologien gehören.

Der positiven Darstellung des mit dem Internet verbundenen Entwicklungspotenzials entsprechend war die Diskussion in der zweiten Hälfte der 1990er Jahre durch eine allgemeine Wachstumseuphorie bestimmt. Unterstiutzt durch staatliche Förderprogramme wurden mehrstellige Millionenbeträge in neue Infrastrukturen und Anwendungen investiert. Doch heute, im Zeitalter kommunaler Finanzprobleme, nach der Krise der New Economy, die zur Insolvenz vieler Unternehmen führte, die für die Kommunen die benötigte Software entwickeln sollten, werden technologische Innovationen unter einem neuen Blickwinkel diskutiert. Nun stehen Effizienzgewinne, Kostenersparnisse und Nützlichkeitserwägungen im Mittelpunkt.

\section{VON „TELEMATISCHEN“ STÄDTEN UND „ELEKTRISCHEM“ KAFFEE - DIE ZUKUNFT DES INTERNET IN DEN KOMMUNEN}

Heute lässt sich absehen, dass in Zukunft das Handeln der kommunalen Gebietskörperschaften in mindestens zweifacher Hinsicht durch das Internet geprägt sein wird:

- Die kommunalen Aufgabenfelder verändern sich in Abhängigkeit von den Anforderungen durch die sich wandelnde Gesellschaft. Neue Kosten- und Wertschöpfungsstrukturen, neue Wettbewerbs-, Vermarktungs- und Kommunikationsstrategien entstehen. Daher verändern sich traditionelle Aufgaben (so bei der Entwicklung virtueller Rathäuser), neue Aufgaben kommen hinzu (z.B. das Engagement der Kommunen beim Aufbau virtueller Marktplätze), andere Aufgaben werden privaten Akteuren übertragen oder zusammen mit privaten Akteuren angegangen (private public partnership). 
- Die Art und Weise des kommunalen Handelns verändert sich. Der Einsatz des Internets zur eigenen Aufgabenwahrnehmung ist mit administrativen, sozialen und ökonomischen Veränderungen verbunden. Die Rolle der Kommune als Dienstleister für Bürger und Wirtschaft wird im Rahmen von E-Governance neu definiert werden.

Der durch das Internet geschaffene virtuelle Raum ergänzt den physischen Ort. Das bedeutet zum Beispiel, dass virtuelle Informationen den Gebrauch lokaler Strukturen unterstützen können, indem sie auf vorhandene Angebote in räumlicher Nähe aufmerksam machen. Enge administrative Grenzen könnten weiter an Bedeutung verlieren, regionale Zusammenhänge könnten demgegenüber wichtiger werden. Der absehbare Zugriff auf ein breiteres Angebot an Bürgerdienstleistungen über das Internet könnte den Druck auf die einzelne Kommune erhöhen, die Qualität ihrer Dienstleistungen zu verbessern.

Grundsätzlich sind und bleiben die Wirkungen der Informations- und Kommunikationstechnologien aber ambivalent: Man kann sie zur Verbesserung der Lebensverhältnisse in den Städten und Gemeinden nutzen, man kann sie aber auch zur Aufrechterhaltung bestehender verkrusteter Strukturen einsetzen. Allein durch die Verlegung von Verwaltungsvorgängen ins Internet werden diese nicht zum Vergnügen für die Bürger. Die Nutzung des Internets macht den Verwaltungsmitarbeiter nicht automatisch zum ,gentil organisateur" der Informationsgesellschaft. Denn letztlich bestimmen immer Akteure über Entwicklung, Anwendung und damit den Erfolg neuer Technologien. Zukunft und Folgen virtueller Rathäuser und Marktplätze in den Städten und Gemeinden hängen also ganz wesentlich von ihren Gestaltern ab.

Von großer Bedeutung für die kommunale Nutzung der Potenziale der neuen IuK-Technik ist, dass lokale Promotoren die Entwicklung vorantreiben, dass Städten und Gemeinden die notwendigen, insbesondere finanziellen, Mittel für die Erneuerung zur Verfügung stehen und dass Akteure aus allen gesellschaftlichen Bereichen in den Erneuerungsprozess eingebunden werden. Dafür muss das technologische Leitbild der ,telematischen Stadt“5 um ein gesellschaftliches Leitbild ergänzt werden. Einige derartige Ansätze liegen bereits vor. Leitbilder, die den gesellschaftlichen Umgang mit neuen Informations- und Kommunikationstechnologien beinhalten, sind aber nach wie vor oft zu technologisch orientiert und gehen bisher nur unzureichend auf die Entwicklung der Beziehungen zwischen „,virtueller" und „materieller" Stadt ein. Das Leben in den Städten und Gemeinden wird durch die verstärkte Nutzung des Internets nicht „ortlos“, sondern „neu verortet“. Die Stadt wird nicht allein dadurch „telematisch", dass die

5 Mit diesem Begriff wurde Mitte der 1990er Jahre der Zusammenhang von Stadtentwicklung und technologischer Entwicklung im Bereich der Informations- und Kommunikationstechnologien beschrieben. Mit ihm war "die Zukunftsvision verbunden (...), dass eine flächendeckende telematische Infrastruktur alle offentlichen und privaten Bereiche digital vernetzt und jeder zu jeder Zeit und an jedem Ort mit jeder anderen Person und Institution über ein technisches Netzwerk multimedial und prinzipiell grenzenlos kommunizieren kann" (Kreibich 1997, S. 6). 
Verkehrsnetze durch digitale Kommunikationsnetze ergänzt werden und Verwaltungsmitarbeiter mit den Bürgern statt per Telefon nun durch das Internet kommunizieren. Kaffee wird ja auch nicht elektrisch dadurch, dass er mit der Kaffeemaschine gebrüht wird.

\section{LITERATUR}

Deutsches Institut für Urbanistik (Difu) (Hrsg.) (2002): Erfolgsmodell kommunales EGovernment, Berlin.

Floeting, Holger (2001): Städte und Regionen im Internet, in: Institut für Länderkunde Leipzig (Hrsg.): Nationalatlas Bundesrepublik Deutschland. Verkehr und Kommunikation, Heidelberg/Berlin, S. 110-111.

Floeting, Holger und Steffi Gaevert (1997): Städte im Netz. Elektronische Bürger-, Stadt- und Wirtschaftsinformationssysteme der Kommunen. Ergebnisse einer Difu-Städteumfrage, in: Deutsches Institut für Urbanistik (Hrsg.): Aktuelle Information, Berlin.

Floeting, Holger und Busso Grabow (1998): Auf dem Weg zur virtuellen Stadt? Auswirkungen der Telematik auf die Stadtentwicklung, in: Informationen zur Raumentwicklung, Heft 1, S. 1730.

Floeting, Holger und Britta Oertel (2002): „Neue Medien“ und Stadtentwicklung. Virtualisierung und Entstehung neuer Raummuster in der Stadt, Berlin.

German, Christiano (1996): Politische (In-)Wege in die globale Informationsgesellschaft, in: Politik und Zeitgeschichte, Bd 32.

Gruppe hochrangiger Experten für gesellschaftliche Aspekte der Informationsgesellschaft bei der EU (HLEG) (1996): Eine Informationsgesellschaft für alle. Erste Überlegungen der Gruppe hochrangiger Experten. Zwischenbericht, Brüssel.

Kreibich, Rolf (1997): Telematic Cities, in: Burmeister, Klaus und Kai Böhme (Hrsg.): Telematic Cities. Perspektiven nachhaltiger Stadtentwicklung, Gelsenkirchen, S. 6-15.

Kubicek, Herbert (1996): Bürgerinformationssysteme. Stand und Perspektiven im Rahmen multimedialer Stadtinformation, in: Floeting, Holger (Hrsg.): Medien, Kommunikation und Stadtentwicklung, Berlin, S. 71-100. 


\section{Der Geocode des Internets}





\title{
ZUM VERHÄLTNIS VON ZENTRUM UND PERIPHERIE IM INTERNET
}

\author{
Christian Stegbauer
}

Es findet sich eine ,häufig beobachtete Eigentümlichkeit komplizierter Gebilde; dass das Verhältnis eines Ganzen zu einem anderen sich innerhalb der Teile eines dieser Ganzen wiederholt" (Simmel 1890, zit. nach 1989, S. 115).

\section{SELBSTÄHNLICHKEIT SOZIALER STRUKTUREN}

Im vorangestellten Zitat geht es Simmel um strukturelle Ähnlichkeiten auf unterschiedlichen Aggregationsebenen. Solche Ähnlichkeiten, die man auch als selbstähnlich bezeichnet, finden sich zwischen einer Vielzahl sozialer Gebilde, die sich strukturell oft auf verblüffendste Weise gleichen. In diesem Beitrag wird gezeigt, dass das Prinzip der Selbstähnlichkeit sozialer Strukturen auch auf das Internet zutrifft.

Dazu werden drei Typen von Aggregationsebenen betrachtet, die sich grob nach der Komplexität ihrer Struktur sowie dem Grad ihrer individuellen Beeinflussbarkeit unterscheiden lassen. Diese drei Ebenen sollen auf das Auftreten von Regelmäßigkeiten hin untersucht werden, die dann in die Theoriebildung einflieBen können. Dabei wird der Blick nicht von vornherein allein auf das Internet gerichtet, auch soll die Schau nicht verengt werden auf die Beziehungen zwischen Internet und Geographie. Ausgangspunkt sind vielmehr allgemeine Regeln der Strukturierung von Beziehungen, die, wie sich zeigt, auch von technischen Kommunikationseinrichtungen noch so komplexer Art nicht überwindbar sind. Die Struktur, die betrachtet werden soll, wird in der geographischen Analyse genau so benannt wie in der soziologischen: Es handelt sich um die Zentrum-PeripherieStruktur.

Die drei unter der Perspektive der Zentrum-Peripherie-Struktur zu untersuchenden Ebenen sind jeweils ineinander verwoben, sie weisen Vermittlungen auf und entfernen sich dennoch immer weiter von der einzelnen Person und ihren Möglichkeiten, Einfluss zu nehmen. Die erste der drei Ebenen ist die „einfachste“, nämlich das Individuum und seine Beziehungen. Sodann wird ein intermediärer Standpunkt eingenommen: Bei internetbasierten Kommunikationsforen (Mailinglisten) handelt es sich um eine Aggregationsebene, auf der der Einzelne zwar über einen gewissen Einfluss verfügt, aber nur noch wenige eigene Steuerungsmöglichkeiten in der Hand hält. Bei der dritten Ebene, die man als Makroebene bezeichnen könnte, handelt es sich um das Beispiel der Verlinkung des gesamten 
Internets. Die drei Ebenen sind nicht immer klar voneinander zu scheiden - wichtig ist aber, dass die Emergenz ${ }^{1}$ mit der Aggregationsebene weiter zunimmt und der Einfluss des Einzelnen immer mehr schwindet.

Zunächst soll ein kurzer Blick auf die Zentrum-Peripherie-Beziehungen geworfen werden, wie sie in der Geographie seit langem bekannt sind und anhand von Siedlungsmustern und der Verteilung von Markt- und weiteren Funktionsbereichen - beispielsweise in der Theorie der zentralen Orte (vgl. Christaller 1933) beschrieben werden. Trotz aller Unterschiede zwischen Kommunikationsnetzwerken und den in der Geographie behandelten Gebilden, erscheint es nützlich, zur Veranschaulichung des Gemeinten beispielhaft eine geographische Zentrum-Peripherie-Struktur, die auch durch das Prinzip der Selbstähnlichkeit gekennzeichnet ist, anzuführen.

Selbst in den kleinsten Dörfern lassen sich schon Zentren und periphere Gebiete unterscheiden. Die Zentren sind meist historisch gewachsen, liegen an Verkehrsachsen und weisen Verdichtungen auf. Ähnliches lässt sich von Städten sagen. Neben der Funktion des Verkehrsknotenpunktes kommen Zentren oft noch weitere, miteinander zusammenhängende Funktionen zu, etwa Verwaltungsaufgaben oder Einkaufsmöglichkeiten. Da diese Funktionen das Zentrum attraktiv erscheinen lassen, strömt der Verkehr ins Zentrum und es wird die Verkehrsinfrastruktur ausgebaut, was das Zentrum noch weiter an Attraktivität gewinnen lässt.

Ein wesentliches Merkmal hinsichtlich der Verbindung von Zentrum und Peripherie ist die Drehscheibencharakteristik des Zentrums. Sie lässt sich so beschreiben, dass die einzelnen Teile des Gesamtsiedlungsgefüges mehrheitlich über das Zentrum verbunden werden (siehe Abb. 1). Würde das Zentrum aus irgendeinem Grunde ausfallen (z.B. durch Sperrung der Wege), dann wären zahlreiche Verbindungen zwischen den peripheren Siedlungsteilen nur noch über Umwege oder gar nicht mehr erreichbar. ${ }^{2}$

In großen Städten finden sich sogar oft mehrere Zentren, in Stadtteilen entwickeln sich Unterzentren, die bestimmte Aufgaben für die umliegenden Wohngebiete erfüllen. Betrachtet man Landstriche, dann wiederholt sich diese Struktur. Haben schon die meisten Dörfer so etwas wie ein Zentrum, so findet sich oft eine kleinere Stadt in der Nähe, die, obgleich selbst schon nach dem ZentrumPeripherie-Muster strukturiert, wiederum eine Zentrumsfunktion für die umliegenden Dörfer innehat. Für mehrere kleine Städte mit ihrem jeweiligen Hinterland fungieren größere Städte als Mittelzentren, die dann Funktionen erfüllen, die in der kleinen Stadt nicht vorhanden oder nicht ausreichend gewährleistet sind.

1 Mit Emergenz wird hier ein qualitativer Sprung aufgrund von Strukturierungen, die durch das Zusammenwirken mehrerer entstehen (oder entstanden sind), bezeichnet, bei dem die Handlungen sich an der Struktur (den Formen) orientieren und die Wirkungen von Handlungen immer weniger vorhersehbar sind.

2 Freilich lassen sich historisch unterschiedliche Gewichtungen zwischen den verschiedenen empirisch vorkommenden Funktionen von Zentren beobachten. So spielten Verkehrsstrukturen sicher nicht immer die Hauptrolle. Oft war beispielsweise die Schutzfunktion von herausragender Bedeutung, was sich daran zeigt, dass so manche Stadt an relativ unzugänglicher Stelle angelegt wurde. Allerdings zeigt sich auch, dass die Zentren in friedlicheren Zeiten wieder an zugänglichere Orte, etwa vor die Stadtmauer, verlegt wurden. 
Mittelzentren wiederum richten sich nicht nur an ihrer Peripherie, sondern auch an den in relativer Nähe gelegenen Oberzentren aus.

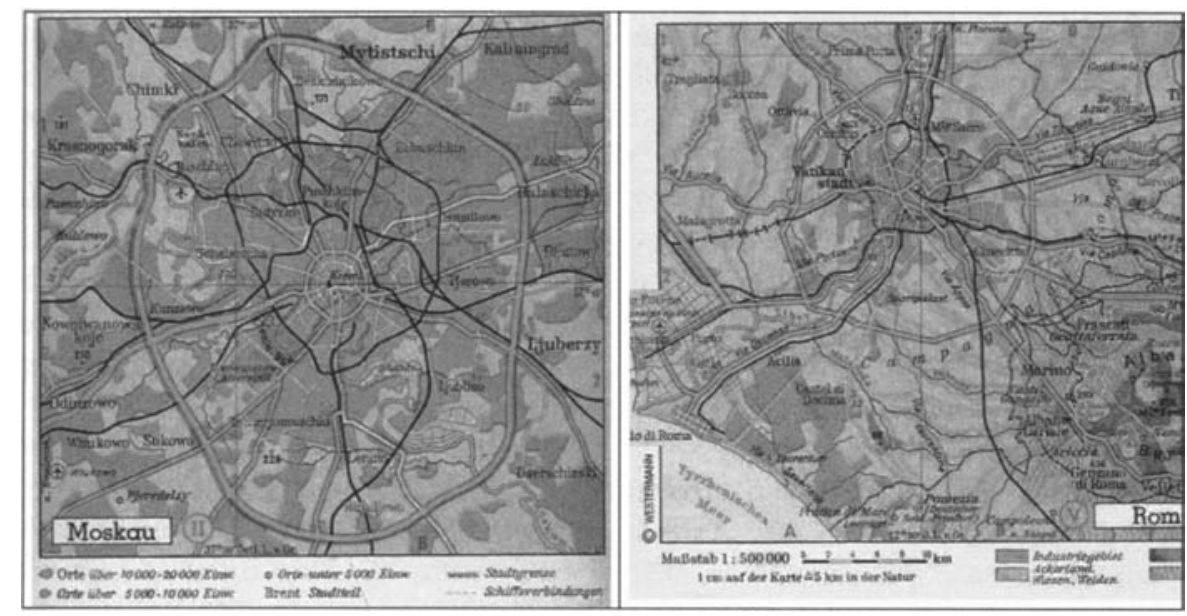

Abbildung 1: Stadtpläne zweier Großstädte aus einem alten Westermann-Schulatlas.

Die Zentrum-Peripherie-Struktur, die schon in der kleinsten Siedlungseinheit, einem Dorf, angelegt ist, wiederholt sich also auf jeder höheren Aggregationsebene. Dies geht so weit, dass viele Staaten zentralistisch auf die Hauptstadt hin ausgerichtet sind, in der dann ein großer Teil der Bevölkerung lebt (Beispiel Frankreich). Nach der Weltsystemtheorie (vgl. Wallerstein 1974) kann man sogar von einzelnen Weltzentren oder Weltmetropolen sprechen (vgl. auch Sassen 1996).

Das beschriebene Grundmuster trifft auch auf die drei nachfolgend zu behandelnden Aggregationsebenen des Internet zu. Die Zentrum-Peripherie-Struktur kennzeichnet nicht nur die Beziehungen oder Konnektivitäten einzelner Personen, sondern, in selbstähnlicher Form, auch Kommunikationsforen und das gesamte Internet.

\section{INDIVIDUELLE EBENE}

Betrachten wir die Beziehungen eines x-beliebigen Menschen (Ego) auf der Welt. Die Beziehungen dieses Menschen lassen sich durch Begriffe beschreiben, in denen ebenfalls geographische Analogien auftauchen. Um nur zwei der gebräuchlichsten zu nennen: Ego verfügt über ihm nahestehende Personen und über entfernte Bekannte. Diese Qualifizierung von Beziehungen mit Hilfe der Entfernungsmetapher wurde von Leopold von Wiese vorgenommen. Er spricht von sozialen Distanzen in einem sozialen Raum, der freilich mit dem physischen 
Raum zusammenhängt, aber nicht als deckungsgleich bezeichnet werden kann (Wiese 1968, erstmals 1924).

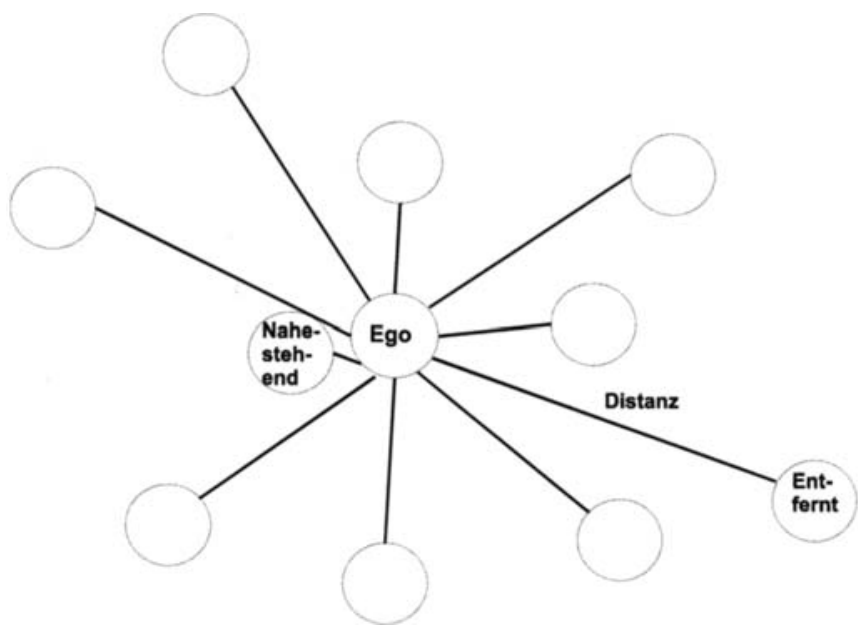

Abbildung 2: Distanzen zwischen Ego und seinen Mitmenschen aus der Sicht von Ego - wie es sich Leopold von Wiese vorstellte.

Aus der Sicht von Ego unterliegen seine Mitmenschen einer sozialen Hierarchie, die sich nach Wiese in Distanzen ausdrücken lässt. Diese lassen sich nicht direkt messen, können aber mittels Indikatoren näherungsweise erschlossen werden. $^{3}$

Die Distanzen zwischen Ego und den anderen Menschen sind aufgrund der damit verbundenen Positionen und Rollenbeziehungen in weiten Teilen nicht frei gestaltbar und auch nicht frei wählbar. Zudem hängen die Distanzen zwischen Ego und seinen Alteri auch von den Beziehungen der Alteri untereinander ab. Ein Problem des Distanzbegriffs besteht darin, dass er nur eindimensional und daher nicht komplex genug ist, um Beziehungen korrekt zu beschreiben. Was vor allem fehlt, ist eine Qualifizierung der Form, in der die Beziehung auftritt. Sind es Beziehungen zu den Eltern, Geschwistern, Freundschaftsbeziehungen, Kollegen, zum Arzt oder handelt es sich um Partnerschaften? Beispielsweise sind Partnerbeziehungen oft enger als die Beziehungen zu den Eltern oder den eigenen Kindern; fragt man aber danach, welche sich eher abbrechen lassen, dann zeigt sich, dass die Beziehungen zu den Eltern/Kindern fast immer auch wechselnde Partnerschaften überleben und somit die größte Stabilität aufweisen (vgl. Hondrich 1997)

3 Wieses Überlegungen bergen ein Problem: Implizit geht er von objoktiv darstellbaren Beziehungen aus; sollten mit Hilfe einer Messung unter Gebrauch von Indikatoren aus Sicht der unterschiedlichen Beteiligten nicht die gleichen Distanzen zu ermitteln sein, muss der Forscher mitteln, bzw. eine Entscheidung treffen. 
Für unsere Zwecke ist das Modell der sozialen Distanzen von Leopold von Wiese aber völlig ausreichend (siehe Abb. 2). Unsere Beispielperson „Ego" steht im Zentrum und um sie herum ordnen sich alle anderen Beziehungen an. Ganz nah werden Partnerin/Partner sein. Ähnlich nahe stehen ihr sicher auch die Eltern, dann mögen die Geschwister und die Freunde kommen. Weiter könnte dann auch noch zwischen Freunden und Bekannten unterschieden werden, wobei Bekannte im Allgemeinen ferner als Freunde erlebt werden. Passanten dagegen sind in diesem Modell schon als vergleichsweise peripher zu bezeichnen.

Ego ist aber auch insofern Zentrum, als zumindest einige der Wege zwischen den ihm bekannten Personen über ihn laufen. Würde Ego als Zentrum aus irgendeinem Grunde (z.B. Umzug oder Tod) ausfallen, entfielen auch die Beziehungen, die vermittelt über ihn ent- und bestehen. ${ }^{4}$

Welche Bedeutung hat nun diese Erkenntnis für die Struktur der durch das Internet vermittelten Kommunikation?

Auch hier zeigt sich, dass die interpersonale Kommunikation und Nutzung des Mediums Internet vorwiegend mit Nahestehenden, sprich Verwandten und Freunden, stattfindet (vgl. Schönberger 1999). Das heißt, das Medium eröffnet zunächst einmal - trotz zahlreicher anderer Möglichkeiten - vor allem zusätzliche bzw. alternative Kommunikationswege zu denjenigen Personen, mit denen man sich sowieso gewöhnlich verständigt. Wo gemailt wird, wird in der Regel auch telefoniert, und wo man derartig enge Kontakte unterhält, sieht man sich auch regelmäßig von Angesicht zu Angesicht - das Medium wird sozial integriert (vgl. Stegbauer 1995). ${ }^{5}$ Barry Wellman konnte an einer Gemeindestudie in Toronto zeigen, dass die wichtigsten persönlichen Beziehungen, zumeist zu Angehörigen, manchmal auch zu ehemaligen Arbeitskollegen und Freunden, auch über weite Entfernungen aufrecht erhalten werden (vgl. Wellman 1996). Dabei spielen neben den Möglichkeiten, die vor allem private Kraftfahrzeuge bieten, natürlich die verschiedenen Kommunikationseinrichtungen (damals vor allem das Telefon, heute zunehmend das Internet) die Hauptrolle. Enge, auch über größere Distanzen beibehaltene Beziehungen machen allerdings nur einen kleinen Teil der Beziehungen des Einzelnen aus. Die meisten Beziehungen werden im Nahraum gepflegt, an dem Ort, an dem man lebt und sich täglich bewegt. Daher kann man schließen, dass die Beziehungsstruktur der Personen, mit denen man per Internet kommuniziert, aus der Sicht von Ego ebenfalls eine Zentrum-Peripherie-Struktur darstellt.

Häufig wurde als eine Folge der Einführung moderner Kommunikationsmedien und insbesondere des Internets der Bedeutungsverlust räumlicher Entfer-

$4 \quad$ Beide Möglichkeiten von Beziehungen fielen weg: Die rein vermittelt bestehenden Beziehungen, in denen Ego Kontakte zu verschiedenen Parteien unterhält, wobei die einzelnen Parteien sich untereinander nicht unbedingt kennen mögen, und zahlreiche solcher Beziehungen, die beispielsweise durch gleichzeitige Besuche, etwa anlässlich von Geburtstagspartys, entstehen.

5 Der Begriff soziale Integration eines Kommunikationsmediums besagt, dass Medien in Beziehungen eingebunden werden. Der Gebrauch und die Anzahl der genutzten Medien hängen von der Beziehung ab. 
nungen konstatiert. Unter Berücksichtigung der vorgetragenen Argumente zeigt sich aber, dass dies für den Einzelnen und seine nach dem Zentrum-PeripherieSchema strukturierten Beziehungen keineswegs zutrifft. Zwar ermöglichen Kommunikationsmedien die Überbrückung des physischen Raumes, vielfach machen sie die räumliche Trennung aber erst schmerzlich bewusst. Selbst dort, wo noch gar keine Beziehungen bestehen, wo das Internet und insbesondere die Chatdienste dazu genutzt werden, neue Beziehungen zu etablieren, scheint es, als zögen die Teilnehmer die Schwierigkeiten der Überwindung des physischen Raumes in Betracht. Sie steigen häufig gar nicht erst in Chatkanäle ein, von denen sie wissen, dass die Teilnehmer weit von ihnen entfernt wohnen. Bevorzugt werden dagegen Kanäle, die Teilnehmer in geographischer Nähe erwarten lassen (z.B. „Kanal Frankfurt"). ${ }^{6}$

\section{EBENE DER INTERNETBASIERTEN KOMMUNIKATIONSFOREN}

Die Betrachtung entfernt sich nun vom Individuum und seinen Beziehungen und richtet sich auf Kommunikationsforen im Internet. Bei diesen Internetforen handelt es sich um sog. Chats, Newsforen oder Mailinglisten. Es wird gezeigt, inwiefern auch die Forenaktivitäten nach dem Zentrum-Peripherie-Muster strukturiert sind.

Als die internetbasierten Kommunikationsmöglichkeiten eingeführt wurden, dachte man, dass endlich eine technische Grundlage dafür gefunden sei, eine gleichberechtigte Teilhabe aller am Kommunikationsgeschehen zu ermöglichen. Es zeigte sich jedoch sehr schnell, dass dies nicht der Fall war. Keineswegs kam es bei Diskussionen im Internet zur ersehnten Gleichberechtigung. Was war der Grund dafür? Offensichtlich schied eine rein technische Erklärung aus. Viel naheliegender schien es, die Gründe für die beobachteten Ungleichheiten bei den Teilnehmern selbst zu suchen: Unzureichende Tastaturkompetenz, mangelnde Fähigkeit, schnell schreiben zu können, ungenügende individuelle Ausdrucksfähigkeit, Kreativität, Hintergrundwissen usw. Derartige Kompetenzdefizite der User waren als Ursachen schnell ausgemacht, um zu erklären, warum sich die erwünschte Gleichheit nicht einstellte.

Empirische Untersuchungen hingegen fanden heraus, dass dies nicht der alleinige Grund dafür sein konnte, dass Ungleichheiten bestehen blieben. Nun wurden unterschiedliche Attitüden der Nutzer beobachtet und beschrieben. Die Gruppe der Nutzer wurden eingeteilt in diejenigen, die sich der elektronischen Kommunikation wie eines Werkzeugs bedienten, in Nutzer, die an der Erforschung der Möglichkeiten und am Zeitvertreib interessiert waren, und in jene Nut-

6 In der Schweiz gibt es Mundartchats, die alle Personen, die nicht dieses spezielle Idiom sprechen (schreiben), ausschließen (vgl. Aschwanden 2001).

$7 \quad$ Eine solche Einschätzung passte sehr gut zu den seinerzeit aktuellen und viel diskutierten Überlegungen einer Diskursethik (Stichwort: deliberativer Diskurs), wie sie etwa von dem Hauptvertreter der kritischen Theorie, Jürgen Habermas, vertreten wurde (siehe hierzu: Peters 1994). 
zer, die sich für die Sache selbst (die Diskussionen und ihre Themen) engagierten (vgl. z.B. Wetzstein/Dahm 1996).

All diese Erklärungsansätze haben zwar eine gewisse Berechtigung, doch sie reichen nicht aus. Indem sie die beobachtbaren Ungleichheiten im Kommunikationsverhalten durch unterschiedliche individuelle Fähigkeiten und Präferenzen zu erklären versuchen, vernachlässigen alle Ansätze mögliche strukturelle Ursachen.

Untersucht man internetbasierte Kommunikationsforen genauer, dann fällt auf, dass, obschon eine größere Anzahl an Autoren auftritt, sich ein Großteil der Beiträge auf nur wenige Teilnehmer konzentriert. Diejenigen, die nichts aktiv zum Kommunikationsgeschehen beitragen, sind immer in der Mehrheit (vgl. Stegbauer/Rausch 2001). Die Mehrheit der aktiven Teilnehmer schreibt nur eine einzige Mitteilung; eine kleine Minderheit dagegen schreibt sehr viele Beiträge und dominiert die Foren. Bei der Untersuchung von 14 unterschiedlichen Mailinglisten summierte sich der Anteil der Teilnehmer, die zusammen mehr als die Hälfte aller Nachrichten beitrugen, auf durchschnittlich 6\% (vgl. Stegbauer 2001a). ${ }^{8}$ Bereits aufgrund dieser Tatsache könnte man von einem Zentrum und seiner Peripherie sprechen: Das Zentrum bilden die Teilnehmer, die eine hohe Anzahl an Beiträgen verfassen, zur Peripherie hin werden es immer weniger Beiträge pro Teilnehmer, bis in großer Entfernung vom Zentrum Teilnehmer auftauchen, die gar nicht forumsöffentlich in Erscheinung treten.

Diese Vermutung lässt sich weiter erhärten, wenn man die in den Foren etablierte Kommunikationsstruktur mit in Betracht zieht. Beziehungen entstehen dann, wenn wechselseitig aufeinander Bezug genommen wird. Dies ist in Kommunikationsforen der Fall, wenn sich z.B. Diskussionen entwickeln oder wenn Fragen beantwortet werden. ${ }^{9}$ Wenn man nun die Struktur der Kommunikation über einige Jahre beobachtet, zeigen sich besonders auffällige und immer wiederkehrende Strukturmerkmale:

Zum einen finden sich Teilnehmer, die zwar einen oder mehrere Beiträge leisten, aber keine Antwort bekommen. In vielen Fällen erwarten diese Teilnehmer auch gar keine Antwort, denn bei ihrem Beitrag handelt es sich lediglich um eine Ankündigung o.ä. Mit der strukturellen Analyse ${ }^{10}$ lassen sich zum anderen die an den Diskussionen aktiv teilnehmenden Personen genauer unterscheiden. Es

8 Verteilungen, bei denen viel auf einen Einzelnen entfällt und wenig auf die Masse, werden unter dem Stichwort „power law" behandelt. Im Gegensatz zur Normalverteilung, die als Glockenkurve bekannt ist, hat eine ,power law"-Verteilung keine Spitze; in einem Histogramm erscheint sie vielmehr als absteigende Kurve.

9 Der Begriff „Beziehung“ wurde in der referierten Untersuchung als gemeinsame Teilnahme an einem Thread definiert (vgl. Stegbauer 2001a). Das heißt, zwischen allen Teilnehmern, die sich zu einem bestimmten Betreff geäußert haben, wird eine Beziehung konstituiert. Diese Definition hat zur Folge, dass die Anzahl der gemessenen Beziehungen mit der Zahl der Teilnehmer eines Threads überproportional zunimmt und dass es sich definitionsgemäß um symmetrische Beziehungen handelt: Bei einer Frage und einer Antwort auf diese Frage entsteht nur eine Beziehung; sind 4 Personen beteiligt, entstehen bereits 6 Beziehungen; sind 10 Teilnehmer beteiligt, entstehen schon 45 Beziehungen usw.

10 Zum Einsatz kamen netzwerkanalytische Instrumente, vor allem die positionale Analyse (Concor-Algorithmus). Näheres hierzu bei: Stegbauer 2001a, Stegbauer/Rausch 1999. 
lassen sich temporär auftretende Multiloge ${ }^{11}$ identifizieren, d.h. Gruppen von Teilnehmern, die sich eine Zeit lang häufiger und an unterschiedlichen Themen beteiligen, später aber nicht mehr oder nur noch sehr selten in Erscheinung treten. Über einen längeren Zeitraum kommt es wiederholt zur Bildung solcher Multiloge, oft treten sie parallel zueinander auf. Die Teilnehmer der Multiloge unterhalten zwar Beziehungen untereinander, nicht jedoch mit anderen Personen, die sich zu dieser Zeit nicht beteiligen, d.h. die einzelnen Multiloge sind gegeneinander relativ abgeschottet:

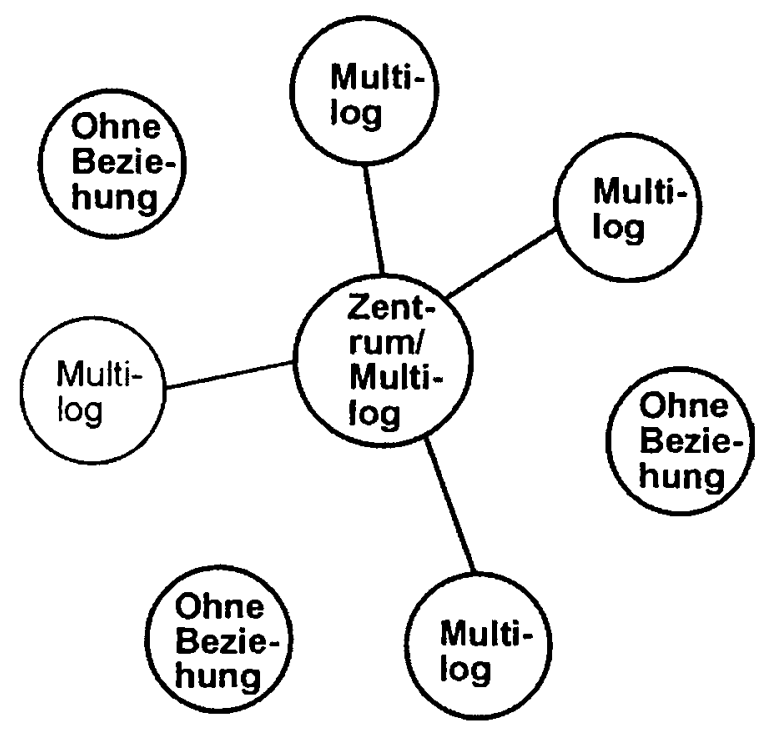

Abbildung 3: Die Kommunikationsstruktur am Beispiel der Mailingliste Critical Café (zur Kommunikationsstruktur von Mailinglisten vgl. genauer: Stegbauer 2001a).

Einzig über ein Zentrum, welches von Teilnehmern gebildet wird, die über einen sehr langen Zeitraum aktiv sind und die sich auch an den einzelnen Multilogen beteiligen, kommt ein Kontakt zwischen den ansonsten isolierten Multilogen zustande. Das Zentrum stellt außerdem so etwas wie ein Gedächtnis des Forums dar. Ohne dieses Gedächtnis wäre die Kommunikationsgeschichte des Forums mit ihren sozialen Leistungen, den geführten Verhandlungen über Verhaltensregeln, den vollzogenen Schließungsprozessen usw., nicht oder nur sehr viel schwächer vorhanden.

11 Der Begriff Multilog wurde von Dialog abgeleitet und bezeichnet einfach, dass eine Vielzahl an Personen (mehr als zwei) an der Kommunikation teilnehmen. 


\section{DAS WWW}

Einzelne Foren sind noch relativ gut zu überschauen. Was ist aber mit dem gesamten Internet bzw. dem Worldwide Web (WWW)? Was kann man überhaupt unter der Struktur des WWW verstehen und wie könnte man sie beschreiben? Finden wir hier nochmals das gleiche Zentrum-Peripherie-Muster?

Ganz allgemein bedeutet „Struktur“ ein Muster von Relationen, d.h. Beziehungen. Diese Relationen kann man auch als ein Netzwerk auffassen. Da man Beziehungen nicht einfach messen kann, ist man auf einen Indikator angewiesen. Gegenseitige Verweise können als ein solcher Indikator angesehen werden. Verweise treten im WWW vor allem als Hyperlinks auf. ${ }^{12}$ Die Links im Internet werden in den meisten Fällen von den Autoren der Seiten zusammengestellt. Nun findet sich aber eine sehr große Bandbreite an Autoren von Internetseiten. Diese reicht von Privatpersonen, die sich einen $\mathrm{Spa} ß$ daraus machen, einen Bericht über ihren letzten Urlaub zu veröffentlichen, über Vereine, die ihre Aktivitäten der Öffentlichkeit bekannt machen, Unternehmen, die etwas verkaufen wollen, Webkataloge und Bibliotheken, die über das Internet zugängliche Zeitschriften zusammenstellen, bis zu Organisationen wie Yahoo, die Redaktionen anstellen, um "gute" Linkquellen anbieten zu können.

Wenn man argumentiert, dass es sich bei der Linkstruktur des Internets um eine Beziehungsstruktur handelt, stellt sich die Frage, ob die Hyperlinks gar als soziale Beziehungen zu betrachten sind. In einem formalen Sinne können Links auf jeden Fall als Beziehungen angesehen werden, denn es wird eine direkte Verbindung hergestellt. Eine soziale Beziehung besteht nun insofern, als beide Seiten - diejenige, von der aus der Verweis stattfindet, und auch die Seite, auf die der Verweis zielt - von Menschen erstellt wurden. Dagegen könnte man einwenden, dass das Merkmal des gegenseitigen Bezugs fehlt und es sich daher sehr oft um einseitige Beziehungen handelt. Doch einseitige soziale Beziehungen sind in der Soziologie nichts Unbekanntes, auch sie wurden schon von Georg Simmel hinsichtlich unbeantworteter Liebe thematisiert. Auf die Spitze getrieben werden solche Beziehungen, wenn z.B. Jugendliche in ihr Idol verliebt sind. Einseitige soziale Beziehungen kommen in der modernen Gesellschaft tatsächlich häufig vor und werden mit den vorwiegend einseitigen und durch hohe Reichweiten ausgezeichneten Massenmedien nur noch wahrscheinlicher. ${ }^{13}$ Sofern es sich bei der verlinkten Seite nicht um eine persönliche Bekanntschaft handelt, sollte man daher von einer schwachen, einseitigen und vermittelten sozialen Beziehung ausgehen.

12 Beim Hyperlink werden bestimmte Stellen eines Textes hervorgehoben, sei es durch eine andere Farbe, Unterstreichung oder durch sogenannte Buttons. Wenn man mit der Maus auf eine solche Stelle im Dokument klickt, dann wird ein Befehl ausgeführt. Solche Befehle sind meist Sprungbefehle. Sie führen auf eine andere Stelle im Dokument, auf mit dem Dokument verbundene Seiten auf derselben Internetsite oder auf ganz andere Sites.

13 Moderne Strukturalisten, wie etwa Craig Calhoun (1991), thematisieren einseitige Beziehungen als soziale Beziehungen am Beispiel von Communities. 
Forscher, die die Struktur des WWW bestimmen wollen, tun dies, indem sie empirisch die Linkstruktur im Web untersuchen. Broder u.a. (2000) führten beispielsweise eine Untersuchung durch, in die sie 200 Millionen Webseiten mit 1,5 Milliarden Links einbeziehen konnten. Solche Untersuchungen, deren Hauptziel vor allem in der Verbesserung der Algorithmen von Suchmaschinen besteht, erbrachten einige für den hier diskutierten Zusammenhang wichtige Resultate. Zu den zentralen Befunden gehört, dass die identifizierte Linkstruktur ebenfalls einem Zentrum-Peripherie-Muster entspricht (s. Abb. 4). Von den untersuchten 200 Mio. Webseiten bilden 56 Mio. das Zentrum (hier SSC - strongly connected component - genannt). Die beiden anderen, aber schon deutlich weniger zentralen Hauptelemente (die „Mittelzentren“) sind das „In“ und das „Out“. „In“ bedeutet, dass man von den Seiten, die in dieser Kategorie zusammengefasst sind, das Zentrum erreichen kann, aber vom Zentrum aus nicht die Seiten dieser Kategorie. Bei der Kategorie „Out“ hingegen verhält es sich umgekehrt: Diese Seiten sind zwar vom Zentrum aus zu erreichen, der Weg von diesen Seiten zum Zentrum ist aber verschlossen. Neben diesen Kategorien enthält die Grafik noch die sogenannten „Tendrils" (die „Peripherie“): Für diese Seiten gilt, dass weder von ihnen aus das Zentrum erreicht werden kann, noch umgekehrt sie vom Zentrum erreicht werden können.

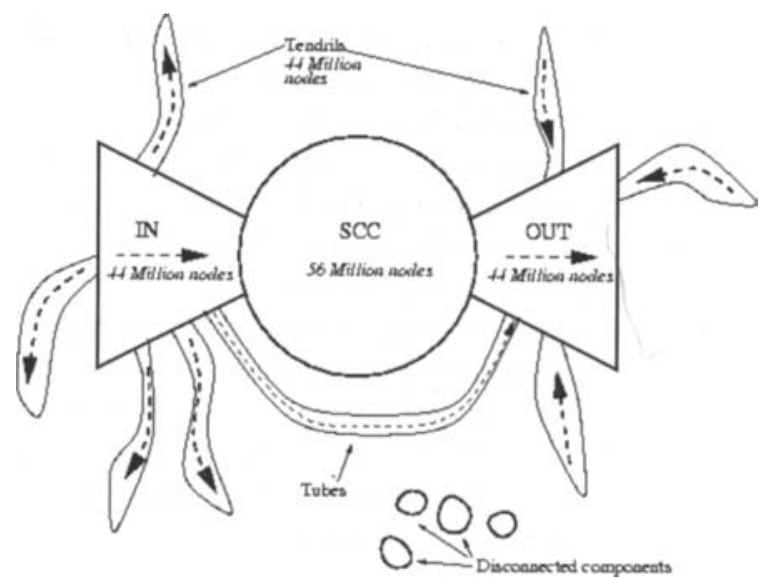

Abbildung 4: Grafische Darstellung der Webstruktur (Quelle: Broder u.a. 2000).

Diese Forschungen fördern noch eine weitere interessante Erkenntnis zu Tage. Betrachtet man kleinere Ausschnitte des WWW, dann fällt eine Selbstähnlichkeit auf - das Web ist fraktalisiert (Dill u.a. 2001): Das Web besteht aus einer Reihe relativ unabhängiger, nach Themen unterscheidbarer Teile. Auch jedes dieser Teile ist durch eine Zentrum-Peripherie-Struktur charakterisiert, die der des gesamten WWW ähnelt. Das Zentrum-Peripherie-Muster wiederholt sich in allen thematisch zuordnungsfähigen Teilen des Web in gleicher Weise. Auch in kleine- 
ren Ausschnitten finden wir immer wieder dieselbe Struktur. Im Verhältnis zum Ganzen können die thematisch zusammengehörigen Teile daher ähnlich wie die Multiloge der Mailinglisten interpretiert werden: An verschiedenen Themen kristallisieren sich hier statt Gruppen von Teilnehmern Gruppen von aufeinander bezogenen Verweisen:

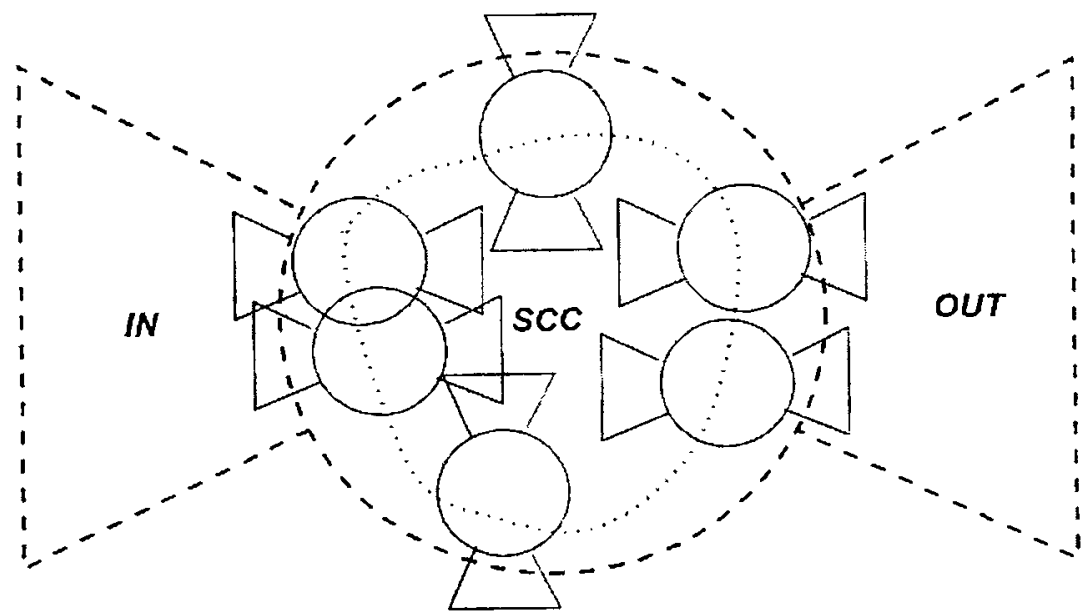

Abbildung 5: Grafische Darstellung der Selbstähnlichkeit im WWW (Quelle: Dill u.a. 2001).

Zentren entstehen im WWW dadurch, dass viele Links auf diese Seiten zeigen. Bestehen erst einmal viele Verbindungen zu wenigen Seiten, dann werden diese Seiten auch am ehesten gefunden und besitzen dadurch die höchste Chance, noch häufiger mit neu eingerichteten Seiten verlinkt zu werden. Ähnlich wie bei persönlichen Beziehungen können bei Neueinrichtungen von Links nur bereits bestehende Sites (und mit ihnen weitere Links) einbezogen werden. Das heißt, auch hier ergibt sich ein Effekt, der deutlich auf der Geschichte beruht.

\section{STRUKTURIERUNGSREGELN}

Es konnte gezeigt werden, dass sich die Strukturen des Internets auf verschiedenen Aggregationsebenen ähneln und den aus der Geographie bekannten ZentrumPeripherie-Strukturen entsprechen.

Charakteristisch für das Zentrum ist seine Funktion, zwischen den unterschiedlichen Teilen des Gesamtgebildes eine Verbindung herzustellen. So würden zahlreiche Bekannte einer Person ohne ihre Vermittlung gar nicht (oder nicht mehr) miteinander in Kontakt kommen. Die Multiloge in den Mailinglisten blieben ohne die Teilnehmer, die die Diskussionen besonders häufig und über einen 
längeren Zeitraum gestalten, unverbunden; was zur Folge hätte, dass die gemeinsam erarbeiteten Diskussionsergebnisse, Konsense und Normen für spätere Diskussionen verloren gingen. Ähnliches gilt für das gesamte Web, in dem die einzelnen Themenbereiche wie Multilogen fungieren; ohne das Zentrum stünden auch diese Themenbereiche unverbunden nebeneinander.

Wie entstehen nun die auf allen Ebenen selbstähnlichen Zentrum-PeripherieStrukturen? Offensichtlich reicht schon eine geringe Zahl von allgemeinen Strukturierungsregeln für ihre Generierung aus:

Auf der Ebene des Einzelnen sind es vor allem (kognitive und zeitliche) Beschränkungen, die dafür verantwortlich sind, dass nicht alle Bekannte, Freunde und Verwandte in gleicher Distanz zu Ego. stehen können. Man kann eben nur Kontakte zu einer gewissen Anzahl von Menschen pflegen. Das bedeutet aber auch, dass Auswahlen getroffen werden müssen, die diese Distanzen begründen. Hinsichtlich der Auswahlgründe würde man zunächst, individualistisch gedacht, Zweck-Mittel-Kalküle unterstellen. Eine wichtige Rolle spielen aber auch Traditionen und kulturelle Formen, die beispielsweise Rollennormen formen und die bei Missachtung zu Sanktionen führen können. Das bedeutet zum einen, dass bei den meisten Menschen bereits ein großer Teil der Kapazitäten, Beziehungen einzugehen, belegt ist. Es können nicht beliebig neue Beziehungen eingegangen werden, weshalb häufig der Wunsch nach eher dauerhaften Beziehungen besteht. ${ }^{14}$ Zum anderen bedeutet es aber auch, dass in Gesellschaften mit einer hohen räumlichen Mobilität die persönlichen Beziehungen, obgleich sie sich fast immer an einem konkreten Ort konstituieren, nach einer gewissen Zeit auseinander gerissen werden. Die meisten Beziehungen gehen nach kürzerer oder längerer Zeit verloren und nur die engsten überleben örtliches Auseinanderstreben. Medien wie das Internet erleichtern zwar die Pflege von Beziehungen über eine größere physische Distanz hinweg. Wenn aber der äußere Anlass für ein Treffen fehlt (Geburtstagsparty, gemeinsamer Arbeitsplatz etc.), lösen sich solche Beziehungen mit der Zeit auf. Insofern ist nicht abzusehen, dass sich die positive Korrelation zwischen der Dauer des gemeinsamen Aufenthalts an einem Ort und der Intensität von Beziehungen durch die Nutzung des Internets aufzulösen beginnt.

Das Problem der Beschränktheit betrifft nicht nur die persönlichen Beziehungen, sondern auch die Kommunikationsstruktur in den internetbasierten Kommunikationsforen. Auch hier kann aufgrund von Beschränkungen nicht jeder mit jedem in Kontakt treten. Nur eine gewisse Anzahl maximaler Kontakte ist möglich. Es ist nicht nur die Anzahl der Kontakte beschränkt. Selbst in den Fällen, wo die Entscheidung über neue Beziehungen auf den ersten Blick „frei“ zu sein scheint, wird sie durch die immer schon bestehenden Beziehungen zu anderen

14 Manchmal besteht eine Notwendigkeit, neue Kontakte einzugehen bzw. alte Kontakte abzubrechen. Dies trifft nicht nur auf einen Umzug $z u$, ein mindestens genau so gutes Beispiel ist es, wenn ein Paar den Übergang von der Zweisamkeit zur Dreisamkeit zu bewältigen hat. Das Neugeborene beansprucht so viel Aufmerksamkeit, dass zahlreiche andere Beziehungen darunter leiden und oft abgebrochen werden. 
(und hier gerade durch die intensiveren, ,freundschaftlichen“ Beziehungen) strukturiert. ${ }^{15}$

Die ,power law" genannte Verteilung, mit der man beschreiben kann, dass wenige Teilnehmer in einem Kommunikationsforum viel und viele wenig oder gar nichts aktiv beitragen, scheint der Erkenntnis der Beschränktheit von Beziehungen entgegen zu stehen. Denn in den Foren unterhalten wenige einzelne Teilnehmer viele Kontakte zu vielen anderen Teilnehmern. Solche Anstrengungen von Einzelnen wären nicht möglich, wenn sie nicht eine Position inne hätten, welche die Erwartungen der anderen Teilnehmer formt. ${ }^{16}$ Es sind also soziale Rollen, in die das Verhalten der Teilnehmer eingebunden ist. Wenn einige Teilnehmer durch ihr Verhalten und durch ihre häufige Präsenz in einem Forum bekannt werden, entstehen bei denjenigen, die auch daran teilnehmen, Eindrücke über ihre Kompetenzen und ihr Verhalten. ${ }^{17}$ In diesem Fall erwarten die anderen Teilnehmer geradezu eine Reaktion, wenn Fragen, Diskussionspunkte oder Normverletzungen den Kompetenzbereich eines dieser bekannten Teilnehmer streifen.

Auch wenn man das gesamte Web betrachtet, treten solche Beschränktheiten auf: Linklisten sind in ihrem Umfang begrenzt, sie beinhalten nur das, was ein Einzelner oder ein Team zusammentragen kann. Autoren von Linklisten überblicken nicht alle Themen vollständig. Aber mehr noch - die besten Suchmaschinen bedienen sich eines Algorithmus, der die Bedeutung einzelner Seiten würdigt. ${ }^{18}$ Die Wichtigkeit wird rein formal nach der Verlinkung gemessen. Für die Suchmaschinen sind solche Seiten bedeutend, auf die von vielen anderen Seiten aus verwiesen wird. Die Rangfolge der auf eine Suchanfrage hin angezeigten Internetseiten wird entsprechend ihrer Verlinkung festgelegt. Wenn viele Links auf eine Seite zeigen, steigt die Wahrscheinlichkeit, dass diese an oberer Stelle zu sehen ist. Damit nimmt aber auch die Wahrscheinlichkeit zu, dass diese von den Nutzern beachtet und mit weiteren Seiten verlinkt wird. Dies führt wiederum dazu, dass die Wahrscheinlichkeit, dass diese Seite gefunden wird, noch weiter steigt. ${ }^{19}$

15 Dies zeigt die sog. Balance-Theorie. Sie geht auf Heiders psychologische Theorie der kognitiven Balance zurück (vgl. neben Heider 1958 auch Cartwright/Harary 1956). In der Soziologie schlossen in den 1970er Jahren zahlreiche empirische Studien an die Balance-Theorie an (vgl. Davis 1977; Davis/Leinhardt 1972; Holland/Leinhardt 1971). Die Balance-Theorie ist eine Hypothese über die Strukturentwicklung von Beziehungen, die darauf aufbaut, dass Beziehungen transitiv sind, d.h. dass ein gewisser Grad an Reziprozität - entweder hinsichtlich einer Freundschaft oder hinsichtlich einer Feindschaft - vermittelt wird (vgl. Stegbauer 2002).

16 Beispielsweise erinnern sich nur sehr wenige Teilnehmer einer Mailingliste ungestützt an die Namen anderer Teilnehmer. In einer von mir durchgeführten Befragung in einer Liste war es sogar nur ein einziger, der mehrmals erinnert wurde und den andere Teilnehmer auch am ehesten kennen lernen wollten (vgl. Stegbauer/Rausch 1999, S. 105).

17 Allerdings ist das Entstehen solcher Zuschreibungen nicht für alle Teilnehmer möglich. Die große Zahl der Beteiligten wird über ein "low profile" nicht hinauskommen.

18 Marktanteile bei Suchmaschinen: Google 55\%, Yahoo 21\% und MSN Search 9\% (vgl. Suck 2003).

19 Ein weiteres technisches Detail verstärkt diese Wirkung zusătzlich: Die meisten Suchmaschinen sind auf eine Auswahl von 10 Sites pro Ausgabeseite voreingestellt. Aus der Marktforschung ist bekannt, dass zwar $100 \%$ der Nutzer die erste Seite lesen, jedoch schon deutlich 
Unser Leben wäre ohne Strukturen gar nicht denkbar. Aus einer schier unbegrenzten Anzahl an möglichen Beziehungen kann nur eine beschränkte Zahl realisiert werden. Im Internet scheinen die Alternativen oft nur einen Klick voneinander entfernt. Die sozialen Bindungen (meist vermittelt über einen Ort), die sonst unsere Beziehungen so stark bestimmen, treten hier ein Stück zurück, sie unterliegen keiner unmittelbaren Kontrolle durch andere. Diese Freiheit suggerierende Situation bedarf einer Orientierung: Wer kennt wen im Forum? Welches ist die beste Site, um Finanzinformationen abzunfen? Solche Informationen werden zwischen anderen kommuniziert und oft durch Medien verstärkt und bestärkt. Es bilden sich Verdichtungen, die zu (kommunikativen) Zentren werden können. Solche Verdichtungen strukturieren dann weitere Wahlentscheidungen. Denn um sich mit anderen verständigen zu können, müssen Zentren gekannt werden, über die auch die anderen Kommunikationspartner am ehesten Bescheid wissen.

Schauen wir uns alle drei beobachteten Ebenen noch einmal zusammen an. Die Zentrum-Peripherie-Struktur taucht in vielen verschiedenen Zusammenhängen auf. Sie ist ungleichheits(re)produzierend und kaum durch andere „gleichberechtigtere" Strukturen zu ersetzen. Wie gezeigt wurde, handelt es sich bei der Zentrum-Peripherie-Struktur zwar nicht um eine für das Internet exklusive Form, wohl aber eignet sie sich zur Beschreibung des Internets auf unterschiedlichen Ebenen - und dies, ohne die Entscheidungen Einzelner genauer berücksichtigen zu müssen.

Auf allen drei Ebenen ist zu erkennen, dass die Geschichte ein wichtiger Faktor ist. Im Falle der personalen Beziehungen sind es die zu früherer Zeit entstandenen Beziehungen, die das Eingehen neuer und zusätzlicher Beziehungen limitieren. Im Falle der Foren trifft Gleiches zu, was sich auch messen lässt (vgl. Stegbauer 2001b). Nur die Beziehungen, die bereits bestehen, können eine Wirkung auf kommende Beziehungen entfalten. Diese Wirkung bedeutet auch, dass Geschichte nicht revidierbar ist, sie baut auf das Bestehende auf und besitzt die Tendenz, das bereits Etablierte weiter zu stärken.

Allerdings wären unsere Beziehungen, unsere Gemeinwesen und auch das Internet ziemlich langweilig, wenn dieser „Vorrang der Geschichte“ der einzige wäre, der Strukturen beeinflusst. Man kann sich zwar nicht von der Geschichte frei machen, aber ruhend auf den bekannten und Sicherheit gebenden Strukturen, gieren wir nach Neuem, nach Neuigkeiten. ${ }^{20}$ Nach diesem Prinzip funktionieren die Medien und das ist auch die Chance für Newcomer, sich zu etablieren.

weniger (23\%) die zweite Seite in ihre Recherche miteinbeziehen, und noch weniger (10\%) die dritte Seite (vgl. Suck 2003).

20 Edgar Orrin Klapp (1978) entwickelte eine Theorie, die versucht, Öffnung und Schließung auf informationstheoretischer Grundlage zu erklären: Schließung bedeutet danach ,gute Redundanz", sie vermittelt Sicherheit und Geborgenheit. Allerdings kehrt sich nach einer gewissen Zeit das Gute um und wird zu „schlechter Redundanz". Diese bringt dann nichts Neues mehr hervor, es kommt zu vielen Wiederholungen und zu Langeweile. Dann ist der Zeitpunkt gekommen, sich erneut $z u$ öffnen, um noch Unbekanntes aufzunehmen und dann wieder mit einer Schließung zu reagieren. 


\section{LITERATUR}

Aschwanden, Brigitte (2001): „Wär wot chätä?“ Zum Sprachverhalten deutschschweizer Chatter, online: http://www.websprache.net/networks/docs/networx-24.pdf

Bourdieu, Pierre und James S. Coleman (Hrsg.) (1991): Social Theory for a Changing Society, Boulder.

Broder, Andrei u.a. (2000): Graph Structure in the Web, online: http://www9.org/w9cdrom/160/160.html

Calhoun, Craig (1991): Indirect Relationships and Imagined Communities: Large-Scale Social Integration and the Transformations of Everyday Life, in: Bourdieu, Pierre und James S. Coleman (Hrsg.): Social Theory for a Changing Society, Boulder, S. 95-121.

Cartwright, D. und F. Harary (1956): Structural Balance: A Generalization of Heider's Theory, in: Psychological Review, Bd. 63, S. 277- 293.

Christaller, Walter (1933): Die Zentralen Orte in Süddeutschland. Eine ökonomisch-geographische Untersuchung über die Gesetzmäßigkeit der Verbreitung und Entwicklung der Siedlungen mit städtischen Funktionen, Jena.

Cooley, Charles H., Angell, Robert C. und Lowell J. Carr (1933): Introductory Sociology, New York/Chicago u.a.

Davis, James A. (1977): Clustering and Structural Balance in Graphs, in: Leinhardt, Samuel (Hrsg.): Social Networks. A Developing Paradigm, New York u.a., S. 27-34.

Davis, James A. und Samuel Leinhardt (1972): The Structure of Positive Interpersonal Relations in Small Groups, in: Berger, Joseph u.a. (Hrsg.): Sociological Theories in Progress, Bd. II, Boston.

Dill, Stephen u.a. (2001): Self-similarity in the Web, online: http://citeseer.nj.nec.com/ dillo1selfsimilarity.html

Heider, Fritz (1958): The Psychology of Interpersonal Relations, New York.

Holland, Paul W. und Samuel Leinhardt (1971): Transitivity in Structural Models of Small Groups. Comparative Group Studies 2, S. 107-124.

Hondrich, Karl Otto (1997): Die Dialektik von Kollektivisierung und Individualisierung - am Beispiel der Paarbeziehung, in: Hradil, Stefan (Hrsg.): Differenz und Integration. Die Zukunft moderner Gesellschaften. Verhandlungen des 28. Kongresses der Deutschen Gesellschaft für Soziologie in Dresden von 1996. Frankfurt a.M./New York, S. 298-308.

Klapp, O. E. (1978): Opening and Closing - Strategies of Information Adaption in Society, Cambridge.

Nadel, S. F. (1957): The Theory of Social Structure, New York.

Peters, Bernhard (1994): Der Sinn von Öffentlichkeit, in: Kölner Zeitschrift für Soziologie und Sozialpsychologie, Sonderheft 34, S. 42-76.

Sassen, Saskia (1996): Metropolen des Weltmarkts. Die neue Rolle der Global Cities, Frankfurt a.M./New York.

Schönberger, Klaus (1999): Internet zwischen Spielwiese und Familienpost. Doing Gender in der Netznutzung, in: Hebecker, Eike u.a. (Hrsg.): Neue Medienwelten. Zwischen Regulierungsprozessen und alltäglicher Aneignung, Frankfurt a.M., S. 249-279.

Simmel, Georg (1890): Über sociale Differenzierung, in: ders.: Gesamtausgabe. Bd. 2 (zitiert nach 1989, Frankfurt a.M., Suhrkamp), S. 109-295. 
Stegbauer, Christian (1995): Electronic Mail und Organisation. Partizipation, Mikropolitik und soziale Integration von Kommunikationsmedien, Göttingen.

Stegbauer, Christian (2001a): Grenzen virtueller Gemeinschaft. Strukturen internetbasierter Kommunikationsforen, Wiesbaden.

Stegbauer, Christian (200lb): Aufmerksamkeitssteuerung durch Schließung am Beispiel von Mailinglisten, in: Beck, Klaus und Wolfgang Schweiger (Hrsg.): Attention please! OnlineKommunikation und Aufmerksamkeit, München, S. 159-174.

Stegbauer, Christian und Alexander Rausch (1999): Ungleichheit in virtuellen Gemeinschaften, in: Soziale Welt, Bd. 50, S. 93-110.

Stegbauer, Christian und Alexander Rausch (2001): Die schweigende Mehrheit - „Lurker“ in intermetbasierten Diskussionsforen, in: Zeitschrift für Soziologie, Jahrgang 30, Heft 1, S. 47-64.

Stegbauer, Christian (2002): Reziprozität. Einführung in soziale Formen der Gegenseitigkeit, Wiesbaden.

Suck, Michael (2003): Ranking-Betrug bei Google \& Co, in: Chip, Heft 2, S. 222-227.

Wallerstein, Immanuel (1974): The Modern World-System, New York.

Wellman, Barry (1996): Are Personal Communities Local? A Dumptarian Reconsideration, in: Social Networks 18, S. 347-354.

Wetzstein, Thomas A. und Hermann Dahm (1996): Die Nutzer von Computernetzen - eine Typologie, in: Rost, Martin (Hrsg.): Die Netzrevolution. Auf dem Weg in die Weltgesellschaft, Frankfurt a.M., S. 37-50.

Wiese, Leopold von (1968): System der Allgemeinen Soziologie als Lehre von den sozialen Gebilden der Menschen (Beziehungslehre), Berlin (4. überarbeitete Auflage, Original von 1924). 


\title{
RAUM-METAPHERN ALS BRÜCKE ZWISCHEN INTERNET- WAHRNEHMUNG UND INTERNETKOMMUNIKATION
}

\author{
Cornelia Becker
}

\section{EINLEITUNG}

„Ist der Raum Grund von Handlungen oder doch nur eine logische Komponente und Annahme (...)? Oder wird er durch kulturelle Codes und soziale Zuschreibungen erst kommunikativ erzeugt und hervorgebracht?" (Maresch/Werber 2002, S. 13). Diese Fragen gewinnen im Zeitalter des Internets neue Dimensionen, da hier die alltägliche Raumvorstellung des Containers nicht mehr zu greifen scheint und möglicherweise Kommunikationsstrukturen entstehen, die eine neue Perspektive auf räumliche Distanzen und Wirklichkeit eröffnen.

Bei Beschreibungen des Internets fällt auf, dass häufig räumliche Begriffe verwendet werden. Neben dem Cyberspace gibt es Marktplätze, Chatrooms, Cafés im Datenmeer, Online-Foren und Links auf der Datenautobahn. Man geht ins Netz und durch Portale in bestimmte Räume hinein. Auch die Fenstermetapher vieler Softwareprogramme transportiert die Vorstellung, dass man im Internet in einen Raum hinein- oder sogar aus ihm herausschauen kann. Der vorliegende Beitrag geht der Frage nach, welche Rolle die Verwendung von Raum-Metaphern im Kontext der Internetnutzung spielt. Dazu werden Erklärungen dafür entwickelt, wie psychisches Erleben von Raum im Internet zu verstehen ist, inwiefern Internetnutzer über ihre Wahrnehmungs- und Kommunikationsleistungen Internet-Räume erst herstellen und wie Raum-Metaphern die Wahrnehmung strukturieren und das Verstehen von bzw. den Anschluss an Kommunikation gewährleisten.

Aufbauend auf der Betrachtung der gängigen Konstruktion des Internets als einem „virtuellen“ Raum im Gegensatz zum „real-materiellen“ Raum werde ich genauer auf die Funktion der Metapher für die Wahrnehmung eingehen. Im Anschluss daran werden die Bedeutung von Raum-Metaphern und der Herstellungsprozess von Raum im Kontext der Internetnutzung an zwei empirischen Beispielen erläutert.

\section{CYBERSPACE ALS PARALLELWELT?}

Internetnutzer wie -forscher unterscheiden häufig zwischen dem virtuellen Raum des Internets und dem materiellem Raum der Realität. Im Folgenden soll zunächst untersucht werden, ob sich dieses Verständnis des Cyberspace als einer virtuellen 
Parallelwelt der „wirklichen“ Welt auch wahrnehmungstheoretisch begründen lässt.

Nach Roth sollte der Wahrnehmungsprozess als subjektive Hypothesenbildung über die Realität und nicht als objektive Abbildung der materiellen Umwelt aufgefasst werden (vgl. Roth 2001). Wahrnehmung ist eine Grundleistung von Lebewesen, die mit Hilfe ihrer Sinnesorgane und Bewusstseinsapparate durch eigensinnige Selektionen und Strukturbildungen auf Änderungen in ihrer Umwelt reagieren. Wahrnehmungsleistungen sind lebensnotwendig, sie dienen der Orientierung und sind Voraussetzung für soziales Lernen.

Der Wahrnehmungsinhalt muss verlässlich sein. Aber es ist für den Menschen als selbsterhaltendes System nicht notwendig, über eine "direkte Abbildung“ der Realität zu verfügen, um einen „realistischen“ Umwelteindruck zu erhalten. „Realität ergibt sich aus dem erkennenden Tun des Beobachters, der Unterscheidungen trifft und somit den Einheiten seiner Beobachtung Existenz verleiht" (Varela, zit. n. Faßler 2001, S. 57f.). Der Hauptanteil der Wahrnehmung ist folglich eine Konstruktion des Wahrnehmungssystems. Dies kann man sich leicht vergegenwärtigen, wenn man an die sinnlichen Eindrücke denkt, die im Traum erlebt werden. Die hier interessierende Konstruktion geschieht über Vorgänge, die unserem Bewusstsein nur begrenzt zugänglich sind. Denn: „Die Komplexität der Umwelt wird zerlegt in Erregungszustände der Sinnesrezeptoren" (Roth 2001, S. 115).

Schauen wir uns anhand dieser Ausführungen nun den virtuellen Raum im Vergleich zum materiellen Raum genauer an. Ein materieller Raum hat Grenzen; er erhält seine Struktur durch die Anordnung von Dingen und Menschen, seine materiellen Substrate (vgl. Löw 2001, Sturm 2000). Im Internet dagegen kann der Raum nicht "wirklich" materielles Substrat beinhalten. Abgesehen von den Hardwareanteilen des Computers handelt es sich bei dem, was man vielleicht dennoch als Raum bezeichnen könnte, um Repräsentationen. Wenn Wahrnehmung aber generell als Hypothesenbildung über die Realität aufzufassen ist, ist es dann nicht gleichgültig, ob es sich beim Wahrgenommenen um „Realität“ oder um ,repräsentierte Realität“" handelt? Man muss sich fragen, ob es im Hinblick auf das Internet überhaupt sinnvoll ist, zwischen Realität und Virtualität zu unterscheiden. Für das Wahrnehmungssystem ist nicht die materielle Erscheinungsform von Räumen entscheidend, sondern erkennbare Muster, übertragbare Eigenschaften, Formen und sozial zugeschriebene Funktionen. Ebenso wie ein materieller Raum ist dann auch das Internet durch Grenzen und Strukturen gekennzeichnet, die in seinem Fall eben durch die Repräsentation von Dingen und Menschen hervorgebracht werden. Aus theoretischer Perspektive ist der Raum des Internet deshalb nicht weniger real als ein materieller Raum. Beide Räume lassen sich wahrnehmungstheoretisch nur als Ergebnis sozialer und psychischer Herstellungsleistungen verstehen (vgl. Roth 1997 und Vernon 1977). In diesem Sinne kommt auch Wertheim zu dem Ergebnis, dass der virtuelle Raum eine räumliche Natur besitzt. Sie beschreibt den Cyberspace als einen Raum des Seins, als kollektives übersinnliches Reich (vgl. Wertheim 2000). Entscheidend ist dabei, dass das emotionale Erleben der Nutzer, mit anderen Menschen verbunden zu sein, die Realität dieses 
Raumes bestimmt. Der Cyberspace ist insofern ein von Menschen selbstgemachter Innenraum. Erst der (kollektive) Herstellungsprozess macht den Raum des Internets - wie alle anderen Räume - real.

Entgegen diesem theoretischen Argument ist die Unterscheidung zwischen materiellen und virtuellen Räumen aus Sicht der Internetnutzer sehr wohl bedeutsam. Kognitiv ist den Nutzern klar, dass sie sich vor dem Bildschirm befinden, wenn sie im Internet surfen. Emotional können sie sich aber durchaus in einem anderen Raum verorten und die fehlende Dingwelt kompensieren. Dies kann zu Dissoziationen in der Wahrnehmung führen, die wiederum die Unterscheidung von virtueller und materieller Welt motivieren mögen. Ein sinnlicher Bezug zur „Realität" und zum eigenen Körper ist zwar wichtig für die erfolgreiche Teilnahme an sozialen Zusammenhängen. ${ }^{1}$ Dies erklärt aber nicht befriedigend, warum im Zusammenhang des Internets gerade dann Raum-Metaphern so häufig auftauchen und eine offensichtlich zentrale Rolle spielen, wenn es um Kommunikationen geht. Zur Beantwortung dieser Frage wird im Folgenden der Funktion der Metapher nachgegangen.

\section{DIE FUNKTION DER (RAUM-)METAPHER FÜR DIE WAHRNEHMUNG}

Metapher bedeutet Übertragung, Übersetzung, und dient zunächst der Umschreibung von Worten (Du bist ein Esel!). Als Tropus (Wendung) wird die Metapher häufig als verkürzter Vergleich genutzt, um zwei Bildfelder miteinander zu verbinden. Wortbedeutung und Äußerungsbedeutung klaffen hierbei auseinander (vgl. Sowinski 1999, S. 125ff.). Im Gegensatz zur Metonymie (ein Glas trinken, Berlin ist der Meinung ...) ist der Zusammenhang nicht kausaler Art, sondern ein Rückgriff auf vorhandene Denkstrukturen, um Unsichtbares sichtbar zu machen. Anders als das Symbol ist die Metapher allerdings kein einfaches Sinnbild für das umschriebene Wort, sondern baut auf nicht trivialen Analogien auf. Die Ähnlichkeit zum beschriebenen Objekt ist nicht unbedingt logisch. Sie baut vielmehr auf kulturellen Wahrnehmungsmodellen auf und setzt neue Ähnlichkeiten. Parallelen werden erzeugt und bieten so eine Anschlussbasis für die Kommunikation.

Auf der Ebene der Wahrnehmung und ihrer Verarbeitung spielen metaphorische Prozesse eine wichtige Rolle. ${ }^{2}$ Die Unanschaulichkeit des begrifflichen Denkens für ein neues Phänomen wie das Internet bringt die Notwendigkeit der Vermittlung mit sich. Häufig sind kognitive Konzepte nicht ausreichend, um ein Phänomen zu verstehen. Durch Anschauung mit Hilfe von sinnlichen Bildern wird eine Brücke zur empirischen Erfahrung geschlagen (vgl. Debatin 1995). Hier stellt sich eine sinnlich erfahrbare Vorstellung ein, die die Verbindung zu kognitiven Konzepten herstellt. Die gestalthafte Metapher regt über die Erzeugung von

Nach Funken und Löw dient der Raum der Absicherung für den Körper (vgl. Funken/Löw 2002). Für die immer sehr konstruktiven Hinweise von Prof. Martina Löw und den Herausgebern sei an dieser Stelle gedankt.

2 Zur Bedeutung der Metapher für das Verstehen unserer psychischen Vorgänge vgl. Gergen 2002. 
Erinnerungsbildern die Verbindung der Gehirnhälften an, so dass eine Einheit der Erfahrung möglich wird. Die sog. Ikonizität der Metapher weist auf die Verbindung von Sinnbild, Sprachbild und Denkbild in einem anschaulichen Modell hin. Die soziale Funktion der Metapher liegt also in der strukturierenden Kraft, der Sinnstiftung.

Metaphern, die mit Bildern der räumlichen Orientierung arbeiten, zählen zu den primären Metaphern, die über Größe, Entfernung und Position eine Strukturierung nach Wichtigkeit vornehmen. Räumliche Metaphern für das Internet zu verwenden, entspricht dem Bedürfnis nach Struktur und Ordnung. Diese Praxis verweist außerdem auf die sinnliche Erfahrung eines neuen Zustands:

Das Internet wird mit unterschiedlichen Raum-Metaphern beschrieben. Mit Meeresmetaphern wie Navigator, Datenmeer, Surfen etc. verbinden die von mir befragten Internetnutzer beispielsweise Freiheit, Abenteuer, Gefahren, Unberechenbarkeit und Imposanz. Bei Highway denken sie eher an Merkmale wie Geschwindigkeit, Zielstrebigkeit und eindeutige Richtung. Portale bieten in den Augen der Nutzer das Gefühl eines beeindruckenderen Eingangs, als es der Begriff der Tür vermag. Foren werden aufgefasst als archaische Umschreibung für ideale Diskursräume; damit stellen sie indirekt eine stärkere Verbindung zu herrschaftlichen Strukturen her als die Bezeichnung Chatroom.

Denkmodelle, die mit Metaphern einhergehen, beeinflussen den Prozess des Verstehens. Unsichtbares wird durch Metaphern sichtbar, das heißt der sinnlichen Erfahrung zugänglich gemacht. Da Sinnesorgane die einzigen Türen des selbsterhaltenden Bewusstseinssystems zur Umwelt darstellen, helfen Metaphern, eine Hypothese über die Realität zu erhalten, die mit Hilfe eines Rückgriffs auf sinnliche Erfahrungen strukturiert wird. Im Falle der Beschreibung des Internets mit Raum-Metaphern liegt die Vermutung nahe, dass die Hypothese über die Realität des Internets mit Eigenschaften verknüpft wird, die auch materiellen Räumen zugeschrieben werden. Denn dies dient nicht nur der individuellen Erfahrbarkeit und damit der Beschreibbarkeit eines Mediums, sondern gleichzeitig der sozialen Interaktion innerhalb neuer Kommunikationsstrukturen, die das Internet bietet. Räumliche Metaphem im Kontext der Internetnutzung können auf diese Weise Denkbilder erzeugen, die einen sinnlichen Bezug des Selbst in dem Medium Internet herstellen helfen.

Im Folgenden werde ich diese These anhand eines studentischen Experiments und eines exemplarischen Interviews belegen. Gezeigt wird, wie die Konstruktion von Räumen im Kontext der Internetnutzung geschieht, welche Eigenschaften von Räumen im Internet erfahrbar werden und welche Rolle dabei Raum-Metaphern, insbesondere für die Herstellung sinnlicher Bezüge, spielen.

\section{EIN EMPIRISCHES PROJEKT ZU DEN RAUMKONSTRUKTIONEN DER INTERNETNUTZER}

In diesem Kapitel möchte ich erste empirische Ergebnisse aus meinem Dissertationsprojekt mit dem Thema „Kommunikation im öffentlichen Raum des Inter- 
net“" vorstellen. Anhand eines netzgestützten Bürgerbeteiligungsforums und eines textbasierten Rollenspiels soll die Wahrnehmung und kommunikative Herstellung von Raum im Internet aus Sicht der Nutzer analysiert werden.

Zunächst werden Textstellen eines Interviews mit einer Expertin für Bürgerbeteiligungsverfahren untersucht. Sie wird im Folgenden mit Frau A. bezeichnet. Frau A. kennt sowohl netzgestützte Verfahren als auch Angebote ohne Netzunterstützung. Das Interview wurde leitfadengestützt als qualitatives Tiefeninterview durchgeführt. Diese Erhebungsmethode sieht vor, die persönlichen Hypothesen der Befragten zu einem Thema zu erfassen und durch vertieftes Nachfragen ihre eigenen Erklärungen ihrer Aussagen hervorzulocken. Sie wurde deshalb gewählt, da sie einerseits eine strukturierende Führung des Gesprächs vorsieht, andererseits den größtmöglichen Spielraum für die Interviewten lässt, um ein komplexes psychisches Erleben zu beschreiben. Die Fragen nach dem Raumverständnis, nach räumlichen Eigenschaften, die fur Diskurse förderlich sind, nach dem Aussehen, der Atmosphäre und den eigenen Konstruktionsanteilen bei der Wahrnehmung bezogen sich sowohl auf "materielle“ als auch auf „virtuelle“ Räume. Sie wurden durch Zwischenfragen ergänzt, die möglichst freie Assoziationen und Vergleiche hervorrufen sollten. Ziel des Interviews war die Hinführung der Interviewten von der Beschreibung ihrer Wahrnehmung zur selbstreflexiven Analyse ihres aktiven Anteils am Konstruktionsvorgang.

Im zweiten Teil werden erste Ergebnisse eines Experiments vorgestellt, das mit einer Studentengruppe durchgeführt wurde. Die Studenten erhielten die Aufgabe, sich in dem Internetspiel Silberland einen Charakter zu schaffen und sich darin zwei Stunden aufzubalten. Anschließend notierten sie ihre Erfahrungen und Gefühle nach vorgegebenen Fragen. Sie wurden nach ihren Schwierigkeiten beim Zurechtkommen in der MUD $^{3}$-Umgebung gefragt, danach, wie sie die Kommunikation erlebt haben und welche Art von Menschen sich wohl vorzugsweise im MUD aufhalten.

Die Auswertung der beiden empirischen Teilstudien erfolgt durch Kategorisierung der Antworten nach metaphorischen Aspekten bei der Beschreibung von Räumen, der Funktion der Raum-Kategorie für die Wahrnehmung und Nutzung des Internetmediums sowie den eigenen Herstellungsleistungen.

\subsection{Die Bedeutung der Raum-Metapher für das Verstehen der Kommunikation}

Die erste Frage des Interviews mit Frau A. bezieht sich auf den öffentlichen Raum im Allgemeinen. Wodurch zeichnet sich öffentlicher Raum aus, worin liegt der Unterschied zu privaten Räumen? Die Antwort beinhaltet zunächst interessanterweise wenig sichtbare räumliche Merkmale. Frau A. definiert den Raum zuerst über die anwesenden Personen, die sich austauschen. Es ist ein Ort, an dem Menschen zusammenkommen. Öffentlicher Raum und privater Raum unterscheiden sich ihr zufolge durch die Zugangsrechte:

3 Multi User Domain; virtuelle Umgebung für Rollenspiele im Internet. 
„Eigentlich ist es aber nur dann öffentlicher Raum, wenn alle hineinkommen. Das Problem ist, dass zusätzliche Beschränkungen herrschen. Man muss die Medien überhaupt handeln können, sich Zugang verschaffen. Und dann muss man diese Räume ja auch überhaupt finden können. Das ist im Internet schwieriger, als den Marktplatz irgendwo zu finden. Da kann man ja ringsherum fragen, im Internet nur gewissen Fachleute. Der Zugang ist insofern halt auch schwieriger, weil die Räume ja nicht beworben sind. Die Zugangsschwelle ist da noch höher, aber das kann sich ja noch verschieben. Wenn irgendwo in Gorleben eine Atommülldemo stattfindet, bin ich ja vielleicht auch ausgeschlossen. "

Im Internet sei der Zugang zu bestimmten öffentlichen Räumen manchmal schwierig, da die Zugangsschwelle zu hoch sei. Hier finden sich bereits zwei Raum-Metaphern, die auf ,materielle“ Räume zurückgreifen, um ,virtuelle“ Räume beschreiben zu können. Der Zugang und die Schwelle vermitteln den Eindruck, das Intemet sei ein Raum, in den man hineingeht und der eine Eingangsschwelle hat, die unterschiedlich hoch sein kann.

\section{„Das Raumgefühl ist wichtig beim Auffinden, beim Zugangfinden."}

Frau A. weist mit dieser Aussage auf ein Raumgefühl hin, das sie im Netz aber gar nicht empfindet, denn sie habe da nie das Gefühl, dass sie in einen Raum hineingehe, wenn sie ein Bürgerforum betrete. Dieser scheinbare Widerspruch ist mit der Funktion der Metapher schnell aufgelöst. Das Denkmodell des Hineingehens in einen Raum hilft, einen Zustand zu beschreiben und zu verstehen, der sonst schlecht auszudrücken wäre. Frau A. ist sich ja bewusst, dass sie vor einem Monitor sitzt. Trotzdem kommuniziert sie aber mit anderen Menschen über den Bildschirm. Die kognitiven und emotionalen Komponenten des Erlebens dissoziieren. Hier wird eine Verbindung benötigt. Frau A. braucht einen Anschluss, den sie leichter über die Metapher des Raumes findet. So nimmt sie sich emotional in einer Kommunikationssituation zusammen mit anderen Menschen wahr, in einem gemeinsam geteilten Kommunikationsraum. Dieser Raum weist darauf hin, dass es anschlussfähige Kommunikationen gibt. Jeder versteht, was gemeint ist, wenn man im Internet in ein Bürgerforum hineingeht. Der Raum, ,in den man hineingeht", vermittelt Sicherheit durch eine überschaubare, überblickbare Situation.

"Und es ist halt auch immer schön, und das gilt halt auch fürs Netz, wenn du dich deiner Umgebung ein Stück weit versichern kannst".

Sicherheit birgt auch die Metapher des Überblicks, die sowohl mit Räumlichkeit als auch mit Sinnen zu tun hat. Etwas überblicken zu können, bedeutet, etwas höher zu stehen als der Rest, oder zumindest muss die Anordnung der Menschen und Dinge im Raum so sein, dass man sie mit einem Blick erfassen kann. Hier ist der sinnliche Bezug der Metapher zur Wahrnehmung direkt zu erkennen. Das Bild des Überblickens dient dem Verstehen einer kognitiven Leistung. Dies gelingt offenbar besser mit Hilfe des Bezugs zu den Sinnesorganen. Besonders der Bezug zur Höhe und Größe birgt ein Verständnis von Ordnungszuständen - wir erinnern 
uns, dass dies primäre Metaphern sind. Strukturen müssen scheinbar über Sinnesorgane hergestellt werden, da es uns so vorkommt, als seien diese dafür zuständig. Für eine neue kommunikative Situation, der Frau A. ausgesetzt ist, wenn sie in eine Diskussion einsteigen will, ist dies ganz ähnlich. Sie will sich zunächst einen Überblick verschaffen.

"Ich glaube, wenn man das Gefühl hat, dass man das gar nicht mehr erfassen kann und überschauen kann, wäre das hinderlich. Zum Beispiel, man würde die Jahrhunderthalle als Diskussionsraum nehmen, was mit Hilfe von Medien natürlich möglich wäre. Trotzdem hätte man das Gefühl, dass man gar nicht mehr folgen kann, weil einfach zu viel parallel läuft, was man nicht mehr überblicken kann und auch nicht mehr zusammenkriegen. Sowohl von den Themen als auch von den Personen. Ich kann dann gar nicht mehr unterscheiden, ob einer schon mal geredet hat oder ob das jetzt ein Neuer ist. Ja dieses menschliche Maß würde da fehlen."

Die Überschaubarkeit eines Diskussionsraums, die für Frau A. so wichtig ist, entspringt einem Bedürfnis nach Sicherheit und Struktur. Das Gefühl, den Überblick zu verlieren, drückt unmittelbar die Angst vor Chaos und Unordnung aus. Auch die folgende Aussage belegt dies:

„Mir ist immer wichtig, dass der Raum hell ist, aber mit Tageslicht, weil das die Diskussion realer macht. Also ein Stück weit sollte das natürliche Umfeld repräsentiert sein. Sonst habe ich das Gefühl, dass ich verloren gehe."

In dem Wunsch nach Helligkeit, in der man alle Beteiligten und alle Vorgänge gut beobachten kann, drückt sich das angesprochene Sicherheits- und Orientierungsbedürfnis aus. Nimmt man bei der Interpretation den anschließenden Nebensatz hinzu, wird noch ein weiterer Aspekt dieses Bedürfnisses deutlich: Die Diskussion soll realer werden, denn Frau A. habe sonst das Gefühl, verloren zu gehen. Die Kommunikation wird also realer für Frau A., wenn die sinnliche Absicherung des Körpers in Bezug zur Umgebung funktioniert. Wenn das Tageslicht vorhanden ist, gelingt es Frau A. leichter, die Diskussion mit ihrer Alltags- und Umwelterfahrung zu verknüpfen:

„Das hat die Sachen, die gesagt wurden, immer ein Stückchen relativiert. Wenn man so theoretische Sachen abhandelt, dann holt das einen immer wieder auf den Boden der Tatsachen. "

Der „Boden der Tatsachen“ weist auf Erdverbundenheit, Schwerkraft, körperliche Existenz hin, die angestrebt wird. Die Gefahr des Sichverlierens in Abstraktion kann durch die Verbindung zur "natürlichen“ Umwelt verhindert werden. Dies ist eine Eigenschaft „realer Räume“, die virtuellen Räumen häufig abgesprochen wird: Die Gefahr des Sichverlierens, des Ausgeliefertseins, des Vergessens der Umwelt. Der sinnliche Bezug zur materiellen Umgebung wirkt dem offenbar entgegen. Man ist der Kommunikation nicht vollständig ausgeliefert und man kann womöglich auch vielfältigere Bezüge herstellen: 
„Das ist so ähnlich wie beim Marktplatz. Man ist den Lichtverhältnissen und dem Wetter ausgesetzt. Man hat auch Einflüsse von außen, Verkehr, Leute, die einfach nur einkaufen. Das ist schon wichtig, dass das auch noch in einem anderen Kontext steht."

Der Vergleich mit einem Marktplatz verdeutlicht, welche Aspekte der Diskussionsraum hier verkörpern soll. Ein Stück Unverbindlichkeit, eine Mischung aus Bekanntheit und Fremdheit sowie die Verbundenheit mit einer weiteren Umgebung als dem unmittelbaren Umkreis scheinen hilfreich zu sein. Auch der mit der Diskussion verknüpften Stimmung nicht vollständig ausgeliefert zu sein, kann hierbei bedeutsam sein - dies obwohl die Stimmung immer auch ein Stück Rückversicherung darüber darstellt, wie die eigene Meinung oder auch Person von anderen bewertet wird. Der Diskussionsraum mit seiner sinnlich erfahrbaren Begrenzung bietet außer der Möglichkeit des kommunikativen Anschlusses also gleichzeitig den Schutz vor diesem Ausgeliefertsein.

„Vielleicht ist ja auch mal eine dunkle Ecke wichtig, wo man sich abschotten kann".

Schutz ist neben dem Anschluss an die Kommunikation eine ebenso wichtige Funktion von Raum. Der beschriebene Diskussions-Raum schützt folglich auch vor dem Chaos der unsortierten Kommunikation. Ist der Ausgang sichtbar, kann man den Raum auch verlassen.

„Es sollte auch eine gewisse Wärme da sein, auch im Sinne von Heizung, dass es eine angenehme Temperatur hat."

Die angesprochene Wärme birgt außer dem tatsächlichen körperlichen Wohlbefinden eine zweite Bedeutungsebene: die menschliche Nähe und die Dynamik einer Diskussion. Eine heiße oder hitzige Diskussion deutet sinnliche Anregung an, aber auch die Gefahr der Entgleisung. Eine Diskussion lässt sich über Temperaturen charakterisieren. Räume vermitteln eine Regelung dieser Temperatur.

Wesentlich für Frau A.'s Raumdefinition ist außerdem, dass diese über die anwesenden Personen vorgenommen wird. Die Einschätzung von Größe und Atmosphäre eines Raumes ist für sie stark davon abhängig, wie viele und welche Personen sich darin befinden.

„Wenn man in einen realen Raum hineinkommt, sieht man sofort, wer darin ist, zum Beispiel 80\% Männer, 20\% Frauen, und man registriert die Stimmung, ob die Atmosphäre angespannt oder locker ist, woraus man ja auch für sich Schlüsse zieht, ob und wie man sich beteiligt."

Hier benutzt Frau A. die Metapher des Spannens und Lockerns von Verbindungen durch Einwirkung von Kräften. Starke Spannung führt zu der Gefahr des Zerreißens. Die kommunikative Verbindung und Nähe könnten zerstört werden.

Die wahrgenommene Atmosphäre des Raums beeinflusst auch die Form der eigenen Präsentation: 
„Das kann natürlich schnell polemisch werden, wenn die soziale Kontrolle fehlt."

Die Möglichkeit, sich vor anderen darzustellen, ist eine weitere wichtige Funktion von Raum, die von der Art und Weise seiner wahrgenommenen Struktur abhängt. Im Internet polemisiert man schneller, da es scheinbar schwächer als "materielle" Räume strukturiert ist und daher die Hemmschwelle niedriger liegt (dies legt auch die medienpsychologische Filtertheorie schon nahe). Wie aber gelangt nun Struktur in einen Raum überhaupt hinein? Offensichtlich reicht Materialität (bzw. der Bezug auf Materialität) allein nicht aus, um strukturierte Räume hervorzubringen. Schauen wir uns daher den Prozess der Herstellung von Raum am Beispiel des Internets einmal genauer an.

\subsection{Der Herstellungsprozess von Raum}

Im virtuellen Raum der Bürgerbeteiligung existiert für Frau A. zunächst überhaupt kein Raumgefühl:

„,Aber so ein Raumgefühl habe ich da nie. In diesen Kinderräumen geben sie sich ja viel Mühe, dass man dieses Gefühl bekommt. Vielleicht liegt das auch an der Generation, aber ich habe immer das Gefühl, ich habe einen Telefonhörer in der Hand und werde vermittelt und dann sprechen da einer oder mehrere. Als Beispiel, wenn ich ein Buch lese, dann habe ich ja auch nicht das Gefühl, dass ich mich in einem anderen Raum befinde und gerade ein Buch lese. Ich befinde mich im Idealfall direkt in den beschriebenen Räumen und habe diese Bilder im Kopf. Das kann man übertragen. Die Bilder, die ich im Internet aufbaue, haben eben auch mit den Inhalten der Beiträge zu tun. Aber dieses extra Raumgefühl habe ich eben nicht. Wenn ich in ein Diskussionsforum gehe, dann sitze ich zu Hause, das ist mir auch relativ bewusst. Wenn die Diskussion jetzt sehr spannend wird, dann merke ich vielleicht nicht mehr, dass ich zu Hause bin. ${ }^{4}$ Dann bin ich in den Diskussionssträngen drin. Ich muss ja den Diskussionen folgen mit Hilfe dieser Printdinger. Ich habe dann im Kopf nicht auch noch den Platz, mir einen Raum vorzustellen, in dem ich mich befinde. Wenn jemand zum Beispiel jetzt von Irland erzählt, dann habe ich sofort ein Bild von Irland im Kopf, aber ich habe nicht das Gefühl, ich sitze jetzt zusammen mit den Personen in einem Raum und spreche über Irland. So ist es ja auch, wenn ich ein Buch lese. Ich habe ja nicht das Gefühl, ich sitze mit dem Autor in einem Raum und er erzählt mir etwas."

Diese Ausführungen klingen so, als wäre bei Frau A.'s Nutzung des Internets keinerlei Raumgefühl vorhanden. Der virtuelle Bürgerbeteiligungsraum wirkt zunächst nicht räumlicher auf die Nutzerin als ein Buch.

"Ich hatte da nie das Gefühl, dass ich in einen Raum hineingehe, wenn ich ein Diskussionsforum besucht habe. Wenn man neu reinkommt, hat man das nicht."

4 Dies erinnert an die psychologische Flow-Theorie, die neuerdings auch auf das Internet angewendet wird. Das Gefühl, eine packende Diskussion zu verfolgen, führt zu einem FlowErlebnis, das die Umgebung vergessen lässt (vgl. beispielsweise Wenzel 2001). 
Die oben beschriebe Art von Raumgefühl, die Raum über Personen definiert, findet aber im Internet trotzdem statt, und zwar in einer metaphorischen, sich erst allmählich und über Interaktion herstellenden Form:

„Ich finde, im virtuellen Bereich kann so eine Raumatmosphäre immer nur Stück für Stück entstehen. Also wenn ich zum Beispiel in ein Diskussionsforum längere Zeit hineingehe, wo ich auch die Personen schon kenne, dann kann ich ein Raumgefühl entwickeln, weil ich weiß, wann die Leute da sind, wie viele Leute da sind, ich kann die Größe einschätzen. Das Ganze kann ich erst über die Zeit hinweg aktiv herstellen."

„Realiter" ist kein Raum vorhanden, die beteiligten Objekte und Personen lassen sich über Wahmehmung nicht direkt räumlich ordnen. Daher, so legen die Ausführungen nahe, ist das Raumgefuihl nur über erlernte Repräsentationen mit einem starken konstruktiven Anteil möglich. Dies schildert Frau A. auch selbst, wenn man sie direkt nach dem eigenen Anteil beim Raumgefühl fragt:

„Natürlich musst du selber viel leisten. Aber das geht nur über die Personen, wenn man sie eine Weile kennt, die stellen ein Raumgefühl für dich her: angenehm, klein, freundschaftlich. (...) Über die Menschen schließe ich zurück auf den Raum."

Wenn die Anordnung von Menschen und Dingen einen Raum bildet (vgl. Löw 2001), dann sind im Internet die Menschen wesentlich wichtiger als Dinge, da den Dingen in der Repräsentation weniger Gewicht zukommt als den Menschen und ihren Kommunikationsbeiträgen. Den Bezug zu dieser Anordnung muss Frau A. allerdings erst aktiv herstellen. Dies gilt interessanterweise in ganz ähnlicher Form auch für ,reale“ Bürgerforen. Auch hier ist nicht so sehr der räumliche Eintritt an einer bestimmten Stelle entscheidend, sondern vielmehr der Zeit= punkt des Eintritts und die Möglichkeit, thematisch-kommunikativ anzuschließen.

„Ja, Struktur ist wichtig für die Orientierung, thematisch, räumlich vielleicht, vor allen Dingen auch zeitlich. Begrenzung ist wichtig. Den Leuten einfach nur einen Platz zur Verfügung zu stellen, reicht nicht, das ist zu wenig. Einfach einen Marktplatz hinstellen und sagen, da könnt Ihr Euch treffen, das ist doch zu wenig. Dann sollen sie wenigstens ein Eiscafé anbieten oder so etwas. Für den öffentlichen Raum, der für demokratische Prozesse dient, brauche ich sozusagen immer jemanden hintendran, der ihn für bestimmte Zwecke nutzen will. Für den demokratischen Raum habe ich wirklich den Anspruch, ein Ziel damit zu verfolgen und da muss ich auch Grenzen setzen."

Die Begrenzung dient u.a. dem Sicherheitsgefühl der Akteure. Nur einen begrenzten Raum kann man auch wieder verlassen. Jedoch reicht die rein materielle Grenze offensichtlich nicht aus. Einen Raum zur Verfügung zu stellen, ist nicht genug. Die Ausfuillung eines Raumes durch gesellschaftliche Praxis ist notwendig, um dem Raum eine Bedeutung, eine gesellschaftliche Funktion zu geben und den Anschluss an die Kommunikation zu gewährleisten. Man muss auch wissen, was man in einem Raum tun kann, erst dann fühlt man sich sicher. Für die Interaktion 
zwischen Menschen ist daher räumliche Struktur als kollektives Gedächtnis auch ausschlaggebend für den Verlauf einer Kommunikation. Denn „materieller“ Raum repräsentiert eine bestimmte gesellschaftliche Praxis, die bereits von anderen Menschen vorstrukturiert ist. Aber die materielle Struktur reicht nicht aus, die Akteure müssen sie auch individuell wieder ausfüllen und sich den Raum aneignen. Erst so stellen Akteure sozial wirksame Grenzen her. Dass die materielle Grenze nicht ausreicht, um Struktur zu schaffen, zeigt die Antwort von Frau A. auf die Frage, was denn furr sie einen leeren Raum ausmache:

„Eine Begrenzung oder Örtlichkeit ist da, aber sonst ist nichts weiter da. Räume werden ja in der Regel gebaut, um da etwas hineinzumachen."

Das, was da „hineingemacht“ werden muss, sind Menschen, Themen, Inhalte, Kommunikationen.

Auch der virtuelle Raum wirkt in gewisser Weise leer, wenn die Funktionen fehlen, die „reale“ Räume bieten oder die mit „realen“ Räumen verknüpft sind.

Diese Erfahrung machen auch die Studenten, die zum ersten Mal ein rein textbasiertes MUD betreten. Auch in textbasierten MUDs gibt es visuell keine räumlichen Anhaltspunkte:

„Da das Ganze auch nicht auf graphischer Grundlage basiert, fiel es mir sehr schwer, mir vorzustellen, wo ich mich gerade befinde. (...) Beschränkt auf kurze Angaben zu einer unsichtbaren, nicht erfahrbaren Umwelt erscheint Orientierung zunächst beinahe unmöglich. “

Die Umwelt ist nicht begrenzt durch räumliche Struktur, die verbalen Beschreibungen helfen Neuanfängern erst einmal wenig weiter. Auch hier entsteht das Gefühl des leeren Raumes und der Unsichtbarkeit. Orientierungslosigkeit fördert entsprechende Reaktionen der Hilflosigkeit, der Frustration. Zumindest anfangs stellt sich sehr schnell das Gefühl der Sinnlosigkeit ein. Es fehlt die Ordnung, die Struktur, die Kenntnis der Funktion des Raumes und der üblichen Praxis. Um dieser verunsichernden Orientierungs- und Strukturlosigkeit mit Ordnungsangeboten konstruktiv zu begegnen, verwenden mittlerweile viele netzbasierten Spiele graphische Elemente wie Containerräume (vgl. Funken/Löw 2002). ${ }^{5}$

Der beschriebene Zustand einer gewissen Hilflosigkeit entspricht dem Gefühl, alles wie ein Kind erst lernen zu müssen:

"Gerade ein Alter Ego (ein anderes Ich) auf die Welt gebracht, benannt und mit einem Gesicht ausgestattet, fühlt man sich selbst wie ein Kind, das erst lernen muss zu laufen, Dinge in die Hand zu nehmen und zu sprechen."

Auch diese Äußerung zeigt deutlich, dass es bei dem zu beurteilenden Spiel an Strukturiertheit fehlt. Der Vergleich mit dem Erleben eines Kindes ist treffend.

s Vgl. zu dieser Thematik auch den Beitrag von Kanwischer in diesem Band. 
Ein Kind muss sich die Vorstruktur und den eigenen Anteil für alle Situationen erarbeiten. Damit ist eine Erfahrung benannt, die sich auch auf das Internet insgesamt übertragen lässt: Die Anforderungen an den konstruktiven Eigenanteil bei der Nutzung sind sehr hoch.

„Die Illusion findet im Kopf statt, die Phantasie der Nutzer muss den Raum zwischen den Buchstaben füllen. Der Geist wird gefordert, er muss sich alles aus dem Nichts vorstellen, konstruieren (...) er schwebt frei."

Interessanterweise taucht auch hier wieder die Raum-Metapher auf. Der Raum muss sich nach Ansicht der befragten Studenten irgendwo zwischen den Buchstaben, also der Kommunikation befinden. Der Anschluss an diese Kommunikation fehlt, wenn man neu in ein MUD eintritt. Dies ist ein beängstigender Zustand, den man ohne sinnlichen Bezug nicht versteht. Daher wird die Unsicherheit bzgl. der eigenen Kommunikationsfähigkeit in räumliche Orientierungslosigkeit übersetzt. Die Metapher erfüllt ihren Zweck, obwohl sogar klar ist, dass der Geist sich alles aus dem Nichts erschaffen muss. Es ist wohl so, dass der kognitiv erfasste Moment der Eigenkonstruktion zwar erkannt, der emotionale Anteil des erlebten Mangels aber trotzdem vorhanden ist.

\section{ZUSAMMENFASSUNG UND SCHLUSSFOLGERUNGEN}

Die eigenen Anteile bei der Raumkonstruktion sind für „virtuelle“ Räume offensichtlicher und stärker erfahrbar als für „materielle“ Räume. Im Prinzip läuft aber bei beiden Raumformen der gleiche Prozess ab. Räume werden hergestellt über die Menschen ihre Kommunikationen und Wahrnehmungen. Der Unterschied zwischen „real-materiellen“ und ,virtuellen“ Räumen liegt darin, dass in Bezug auf die Anordnung von Dingen und Menschen ,reale" Räume stärker vorstrukturiert sind und einen geringeren Eigenanteil an Konstruktionsarbeit des Individuums erfordern als ,virtuelle“ Räume.

Dies wirkt sich auch auf der Ebene des emotionalen Erlebens aus. Aus dem Interview mit Frau A. wird deutlich, wie wichtig die konstruktiven Anteile der Raumwahrnehmung sind und wie stark die sinnliche Erfahrbarkeit von Ordnung darauf Einfluss nimmt, wie Akteure Kommunikation erleben. Emotional führt das Fehlen einer Vorstrukturiertheit der Kommunikation (in Räumen) zu einem Gefühl der Unsicherheit. Raum scheint insofern weniger für die Kommunikation an sich bedeutsam als für die an der Kommunikation beteiligten Bewusstseinssysteme der Menschen und ihre Umwelt- und Kommunikationswahrnehmung. Auf Fragen an die Untersuchungspersonen nach den Eigenschaften, die ein Raum haben sollte, erfolgen Aussagen über die Anordnung, die Atmosphäre und die Assoziationen, die über diese Atmosphäre hergestellt werden können. Wir erinnern uns an die Bedürfnisse nach Sicherheit, Orientierung, Flexibilität und Struktur, aber auch an die Verbindung zur natürlichen Umgebung. Diese Aspekte beschreiben Funktionen, die üblicherweise materielle Räume erfüllen. Es werden in 
erster Linie die Überschaubarkeit, der Zugang, die Präsentation des Selbst und die Zusammenführung von Menschen genannt. Das bedeutet, dass für die Einzelnen diejenigen Anteile eines Raumes wichtig sind, die die Kommunikation als gesellschaftliche Praxis ermöglichen. Gleichzeitig ist die Abgrenzbarkeit des Selbst über die Sinne durch die Raum-Wahrnehmung eine Rückversicherung gegen das Ausgeliefertsein an die Eigendynamik der Kommunikation. Die Strukturierung der materiellen Räume und damit die sinnliche Brücke zwischen dem Selbst und der Umwelt fehlen im Internet.

Im Bewusstsein der Internet-Nutzer handelt es sich daher bei virtuellen Räumen nicht um Räume im klassischen Sinn. Das Internet erscheint ihnen nicht räumlicher als das Telefon oder ein Buch. Für diese beiden Medien verwendet man keine Raum-Metapher. Bisher hält sie sich aber für das Internet hartnäckig. Um gesellschaftliche Interaktion zu ordnen und sich glaubwürdig zu inszenieren, scheint es also notwendig, räumliche Metaphern zu verwenden. Vergleichen wir die Funktion der Metapher und die Funktion von Räumen, so fällt auf, dass sie sich sehr ähnlich sind. Metaphern besitzen wie Räume eine orientierende und erwartungsstrukturierende Funktion.

Aus Sicht der Sprachwissenschaftler drückt die Verwendung von Metaphern einen sprachlichen Mangel aus. Eine Metapher drückt etwas aus, das anders nicht gesagt werden kann. Sie wird verwendet, um den Anschluss an die Kommunikation zu gewährleisten. Auch Räume ermöglichen Kommunikation. Außerdem ermöglichen sowohl Metaphern als auch Räume eine Verbindung zwischen Kommunikationen bzw. der gesellschaftlichen Realität und den Sinnen. Neue Zustände brauchen bildhafte Übertragungen, um sie dem Selbst sinnlich erfahrbar zı machen. Ordnung wiederum ist nur anhand des Wahrnehmungsapparates mit seinen Sinnesorganen möglich. Metaphorische Zuschreibungen helfen daher, über sinnliche Anknüpfungspunkte einen neuen Zustand zu ordnen. Nichts anderes sagt die Erkenntnis, dass Metaphern Unsichtbares sichtbar machen. Altbekannte Eigenschaften von altbekannten Dingen werden verwendet, um neuen Dingen bestimmte Eigenschaften zuzuschreiben. Die Verwendung von Raum-Metaphern ist folglich eine doppelte Sicherung der Ordnungsleistung. Die Raum-Metapher hilft im virtuellen Bereich das auszugleichen, was an Vorstruktur fehlt. Gleichzeitig ermöglicht sie die Überwindung der Diskrepanz zwischen emotionalem und kognitivem Erleben. Die Verwendung der Metapher des Raumes im Kontext der Internetnutzung ersetzt die sinnlich erfahrbare Vorstrukturierung und Begrenzung, die in materiellen Räumen schon vorhanden ist. Es handelt sich gewissermaßen um eine Hilfsfunktion zur Kompensation eines Mangels.

Ich bin aber der Überzeugung, dass dieses Phänomen nicht die Folge eines sprachlichen Mangels ist, sondern vielmehr auf das Problem des Verstehens von Kommunikation reagiert. So ist es kein Zufall, dass die Begriffe Verstehen und Begreifen oft synonym verwendet werden. Verstehbar scheint nur das, was den Sinnen unmittelbar zugänglich ist. Es ist deshalb durchaus denkbar, dass RaumMetaphern nur übergangsweise nötig sind, um die neue Kommunikationsform des Internets zu erklären und so symbolisch mit Strukturen und Grenzen zu versehen. Darauf deutet auch die Beobachtung hin, dass eine angemessene Vorstrukturie- 
rung der neuen Kommunikationssituation in speziellen Internetbereichen auch durch neue, veränderte Formen der Selbstpräsentation möglich ist (vgl. Turkle 1995). Ob allerdings die sinnlichen Bezüge durch nicht-räumliche Formen der Strukturierung wirklich zu kompensieren sind, bleibt abzuwarten.

\section{LITERATUR}

Debatin, Bernhard (1995): Die Rationalität der Metapher, Berlin.

Faßler, Manfred (2001): Netzwerke, München.

Funken, Christiane und Martina Löw (2002): Ego-Shooters Container. Raumkonstruktionen im elektronischen Netz, in: Maresch, Rudolf und Niels Werber (Hrsg.): Raum - Wissen Macht, Frankfurt a.M., S. 69-91.

Gergen, Kenneth (2002): Konstruierte Wirklichkeiten, Stuttgart.

Löw, Martina (2001): Raumsoziologie, Frankfurt a.M.

Maresch, Rudolf und Niels Werber (2002) (Hrsg.): Raum - Wissen - Macht, Frankfurt a.M.

Roth, Gerhard (1997): Das Gehim und seine Wirklichkeit, Frankfurt a.M.

Roth, Gerhard (2001): Denken, Fühlen, Handeln, Frankfurt a.M.

Sowinski, Bernhard (1999): Stilistik, Stuttgart.

Sturm, Gabriele (2000): Wege zum Raum. Methodologische Annäherungen an ein Basiskonzept raumbezogener Wissenschaften, Opladen.

Turkle, Sherry (1995): Leben im Netz. Identität im Zeichen des Internet, Reinbek.

Vernon, M. D. (1977): Wahrnehmung und Erfahrung, München.

Wenzel, Olaf (2001): Webdesign, Informationssuche und Flow: Nutzerverhalten auf unterschiedlich strukturierten Websites, Lohmar u.a.

Wertheim, Margaret (2000): Die Himmelstür zum Cyberspace. Eine Geschichte des Raumes von Dante zum Internet, Frankfurt a.M. 


\section{Räume des Internets}





\title{
SOZIALITÄT IM CYBERSPACE
}

\author{
Hubertus Niedermaier und Markus Schroer
}

\section{RAUM OHNE BODEN}

Sowohl aus technischem als auch aus geographischem Blickwinkel ist die Bezeichnung ,Internet' überaus plausibel. Zusammengesetzt aus, international network' bezeichnet es die weltweite Verknüpfung von Computernetzen. In technischer Hinsicht ermöglichte das ARPANet, der Vorläufer des Internet, erstmals die Verknüpfung zweier oder mehrerer Rechner, ohne dass einer zum ,Terminal' des anderen degradiert wird. Damit entstand ein dezentrales Netzwerk, das den Datenaustausch zwischen gleichrangigen Computern zuließ, so dass die Kapazitäten verschiedener Großrechner miteinander verschränkt werden konnten. Das USMilitär, das den Grundstein für die Entwicklung des ARPANet legte, versprach sich von der dezentralen Struktur, dass auch beim Ausfall einiger Standorte im Kriegsfall das Netzwerk insgesamt funktionstüchtig bleibt. Die ersten zivilen Nutzer des ARPANet, die US-amerikanischen Universitäten, profitierten davon, fortan ihre Großrechner ,kooperieren“ lassen zu können (vgl. Winter 1998; Runkehl u.a. 1998, S. 9ff.).

Spätestens mit der rasanten weltweiten Verbreitung des Internet nach der Entwicklung des WorldWideWeb-Browsers 1992 nahm es auch in erdräumlicher Hinsicht den Charakter eines Netzes an. Ähnlich wie ein Spinnennetz umspannt seine Infrastruktur, also die via Glasfaserkabel miteinander verbundenen Server, heute den Globus. Über Ländergrenzen hinweg findet der Datenaustausch innerhalb von Sekunden statt, gleichgültig wie groß die Entfernung sein mag. Die ,Geographie' des Internet gliedert sich nicht in durch Grenzlinien voneinander geschiedene Territorien; sie setzt sich im Gegensatz dazu aus miteinander verknüpften Servern, also aus Punkten, zwischen denen Verbindungslinien verlaufen, zusammen. Während die politische Landkarte wie ein Puzzle aussieht, gleicht die Internet-Karte eben einem Netz. ${ }^{1}$

Dieses lässt sich in seiner technischen und geographischen Formation in zwei Dimensionen darstellen. Nicht erklären lässt sich aber die zunehmende Popularität der Räumlichkeit implizierenden Bezeichnung Cyberspace (vgl. Bollmann 1995), die, zurückgehend auf einen Roman von William Gibson (1987), zunächst lediglich für computergenerierte virtuelle Realität stand, heute aber zumeist die Welt im WWW meint. Assoziationen mit Dreidimensionalität und Räumlichkeit erfreuen sich zunehmender Beliebtheit, haben ihren Ursprung aber wohl kaum in

Vgl. zur Illustration den „Atlas of Cyberspace“ (http://www.cybergeography.org/atlas/atlas. html) sowie den Beitrag von Heinze in diesem Band. 
der technischen oder geographischen Struktur des Web. Nicht der kühle Blick auf die globale Infrastruktur scheint für die Konjunktur des Wortes ,Cyberspace verantwortlich zu sein, sondern der Blick des Anwenders auf den Bildschirm seines PCs zu Hause. Was der User dort zu Gesicht bekommt, ist kein Internet: weder network, noch international.

Internationalität setzte das Überschreiten von staatlichen Grenzen voraus, doch genau diese lässt das Netz verschwinden. Nicht eine territoriale Gliederung bestimmt den Cyberspace, sondern nur virtuelle Punkte: IP-Adressen. Man weiß nicht, ob und wann man Landesgrenzen überschreitet (es gibt keine Schlagbäume und keine Passkontrolle); diese stellen auch nicht die eigentlichen Hindernisse für die Bewegungsfreiheit im Internet dar. In Anlehnung an Ulrich Beck $(1997,1998)$ könnte man von einem ,Transnet' sprechen, denn damit würde ausgedrückt, dass es sich nicht um eine Einrichtung auf zwischenstaatlicher Ebene handelt, sondern man sich jenseits des nationalstaatlichen Rahmens bewegt. Anstatt staatliche Grenzen einfach nur zu überwinden, lässt das Netz diese gleich so gut wie verschwinden. Von Grenzenlosigkeit kann deshalb aber keine Rede sein, sondern die Grenzziehung fügt sich lediglich keiner territorialen Gliederung. Andere Mechanismen des Aus- und Einschlusses etablieren sich im Web: Es werden Gebühren erhoben, Intranets eingerichtet, nur autorisierten Personen via Passwörtern $\mathrm{Zu}-$ gang gewährt usw. Das Internet ist also keineswegs ein öffentlicher Raum bis in den letzten Winkel, sondern wie im Leben sonst auch, bleibt so manches einigen wenigen vorbehalten.

Ebenso wenig, wie sich das Internet dem Surfenden als international darbietet, scheint ein Netzwerk am Monitor auf: Man hat keinen Schaltplan vor sich, sondern surft durch ein Meer aus Texten, Bildern und Klängen; irgendwo beginnend klickt man sich von einer Seite zur nächsten. Man manövriert durch ein Labyrinth von Verästelungen, ohne je das Ganze zu sehen zu bekommen. Man sieht sich nicht einem Netz gegenüber, sondern geht ins Internet; man ist mitten drin - wodurch das Netz letztlich unsichtbar wird.

Als technologischer und geographischer Begriff mag ,Internet" somit durchaus sinnvoll erscheinen, für den gewöhnlichen User bleiben jedoch sowohl dessen Technik als auch Geographie weitestgehend verborgen. Glasfaserkabel und Server bekommt man beim Surfen nicht zu sehen, für gewöhnlich verschwendet man nicht einmal einen Gedanken an sie. Das Fesselnde ist nicht die Infrastruktur des Internet, sondern die virtuelle Welt des Cyberspace. Dieser Begriff fungiert geradezu als Symbol für das, was die Popularität der Internet-Technik ausmacht. Deshalb soll im Folgenden von "Internet' die Rede sein, wenn die technische Infrastruktur oder ihre geographische Ausbreitung gemeint ist, mit ,Cyberspace“ soll hingegen der sogenannte virtuelle Raum bezeichnet werden, in dem der Anwender, surft".

Letzteres bringt zum Ausdruck, wie diese neue Technologie unsere alltagsweltliche Vorstellung vom Raum als physische Größe ins Wanken gebracht hat: Erstens ist der Cyberspace ein Raum ohne (physische) räumliche Ausdehnung. Man kann ihn nicht vermessen und doch kann man sich in ihm bewegen, sogar surfen. Es gibt keine Möglichkeit und macht auch keinen Sinn, die Entfernung 
zwischen zwei verschiedenen Punkten darin in Metern oder Meilen angeben zu wollen, die Angabe der Anzahl an Klicks, die man vom einen zum anderen benötigt, hat hingegen durchaus Informationswert. Ohnehin verwundert es, dass ein zweidimensionaler Bildschirm, an dem sich für den User das ganze Netz-Geschehen abspielt, Assoziationen an Räumlichkeit erweckt. Höhe und Breite sind gegeben, die Tiefe des Raums aber wird offenbar durch die so genannten Hyperlinks ins Spiel gebracht. Durch sie kann man immer weiter in den Cyberspace vordringen.

Zweitens ist der Cyberspace ein Raum ohne geographische Orte. Ebay ${ }^{2}$ ist ebenso sehr bzw. ebenso wenig in San Francisco wie in Frankfurt oder Tokyo. Auch gibt es keine passenden Angaben hinsichtlich geographischer Länge oder Breite, nichtsdestotrotz lassen sich Punkte im Cyberspace orten. Dies geschieht allerdings nicht mittels Informationen über die Position im physischen Raum, wie etwa mit Hilfe von geographischer Länge und Breite, stattdessen besteht im Netz die einzige Möglichkeit etwas aufzufinden darin, die genaue Adresse ${ }^{3}$ in Erfahrung zu bringen. Man gibt die Adresse ein und die gewünschte Website erscheint am Bildschirm. Der Mensch bewegt sich nicht mehr zum Ort, der Ort kommt gewissermaßen zum Menschen. Damit verliert der Ort ein wesentliches Charakteristikum: seine Fixierung im physischen Raum. Normalerweise geht man davon aus, dass Orte eindeutig sind - es gibt nicht den selben Ort an verschiedenen Stellen. Anders im virtuellen Raum: Was am eigenen Monitor erscheint, kann ohne Weiteres gleichzeitig auch an anderen angezeigt werden. Sites lassen sich zwar mittels Adressen orten, aber sie haben keinen festen Ort mehr. Diese ,Ortlosigkeit" macht auch die Rede von der Virtualität plausibel: Im Cyberspace hat nichts einen Ort und doch ist potentiell alles, was er zu bieten hat, überall anzutreffen. Im virtuellen Raum gibt es keinen begrenzt verfügbaren Boden, der aufgeteilt werden muss, damit alles seinen Platz hat. ${ }^{4}$ Die ,Ortlosigkeit ${ }^{\star}$ schafft einen Raum ohne Boden.

Trotzdem ist es verwunderlich, dass der Begriff ,Cyberspace', bei dem Virtualität und Raum kombiniert werden, so weite Verbreitung gefunden hat, schließlich sind wir es im Alltag gewohnt, bei Räumen und Orten an physische und materielle Gegebenheiten zu denken, was deren Virtualität geradezu auszuschließen scheint. Die Notwendigkeit, den Cyberspace ins Reich der Virtualität zu verbannen, tritt zwar deutlich vor Augen, will man Konfusion mit dem alltagsweltlichen Raumbegriff vermeiden, doch warum weckt das Netz überhaupt Assoziationen an räumliche Arrangements? Um das zu verstehen, genügen offenbar weder technische noch geographische Überlegungen. Auf der Grundlage einer soziologischen Argumentation werden deshalb im Folgenden zweierlei miteinander verknüpfte Leitfragen verfolgt: Einerseits geht es um das Problem, ob und wie sich der Cyberspace als (Kommunikations-)Raum begreifen lässt, inwiefern also eine Analyse sozialer Prozesse verständlich machen kann, weshalb elektronische

Gemeint ist das virtuelle Auktionshaus mit der Adresse http://www.ebay.com

Im Englischen bezeichnenderweise „location“ genannt.

Zwar ist Speicherkapazität prinzipiell beschränkt, doch herrscht im Netz (noch) keine Knappheit, die zum Ausschluss zwingen würde. 
Netze im Alltag als Räume aufgefasst werden. Andererseits stellt sich daran anschließend zwangsläufig die Frage, wodurch diese Räume gekennzeichnet sind, wie sich Kommunikation dort strukturiert.

Dementsprechend sollen im nächsten Abschnitt (2.) zunächst einige allgemeine Überlegungen zur sozialen Konstitution des Raumes und des Cyberspace angestellt werden, bevor dann in den nachfolgenden beiden Abschnitten auf zwei spezifische Charakteristika von Sozialität im Netz eingegangen wird: Zum einen (3.) wird behandelt, inwiefern beim Cyberspace von einer fragmentierten Öffentlichkeit gesprochen werden muss; und zum anderen (4.) werden Entwicklungen beleuchtet, die als Soziales ohne socius bezeichnet werden können.

\section{DER ORT DER KOMMUNIKATION}

Sobald Kommunikation nicht mehr auf Anwesenheit der Beteiligten beruht, also mittels dazwischengeschalteter Technik mitgeteilt wird, werden die Spielräume in zeitlicher und/oder räumlicher Hinsicht vergrößert. Das gilt bereits für die ersten Inschriften in Tonscherben oder Steintafeln, genauso aber natürlich auch für Briefe, Bücher, Telekommunikation, Hörfunk, Fernsehen oder Musik-CDs. In all diesen Fällen wird das Mitteilungsverhalten vom Verstehen zeitlich und/oder räumlich auseinandergezogen. Das Internet bietet allenfalls eine Radikalisierung dessen an. Während beispielsweise beim Fernsehen Ausstrahlung und Empfang synchron zu erfolgen haben, dafür aber große Entfernungen überbrückt werden können, sind Grabinschriften relativ zeitbeständig, aber in der Regel ortsgebunden. Das Internet legt nichts dergleichen fest, sondern lässt verschiedene Kombinationsmöglichkeiten offen: Es kann ebenso synchron wie asynchron kommuniziert werden und es macht keinen Unterschied, ob man eine Email ins Büro nebenan oder ans andere Ende der Welt schickt. Auch die Möglichkeit, Text, Bild (bzw. bewegte Bilder) und Ton miteinander zu verbinden, ist nicht die eigentliche Innovation, man denke nur an das Fernsehen oder an Postkarten, wo Text, Bild und im Falle der Parfümierung sogar Gerüche (davon ist das Internet noch weit entfernt) kombiniert werden können.

Dass soziale Beziehungen sich durch Mediatisierung ändern, darauf hat unter anderem Vilém Flusser hingewiesen: Da simultane Anwesenheit für einen Austausch mit den Mitmenschen heutzutage nicht vonnöten ist, kann man seine Kontakte auch über tausende Kilometer Entfernung pflegen. Man kann Geschäfte tätigen, Neuigkeiten austauschen und sich verlieben, ohne einander je gesehen oder gehört zu haben. Man kann sich mit Menschen am anderen Ende der Welt eng verbunden fühlen und zugleich seinen Nachbarn nicht kennen, von dem man nur durch zwanzig Zentimeter Ziegelstein getrennt ist. „Nähe ist [..] nicht Funktion irgendeiner räumlichen und zeitlichen Entfernung, sondern Funktion der Zahl und Intensität der Beziehungen, die den einen mit dem anderen verbinden“, folgert deshalb Flusser (1995, S. 18). Mit anderen Worten: Medien heben die Korrelation von räumlicher und sozialer Nähe auf.

Ebenfalls vorausgeeilt sind andere Medien dem Internet hinsichtlich der Los- 
lösung der Zurechnung von Kommunikation zu einem geographischen Ort: Netzkommunikation findet, wie bereits angesprochen, an mehreren Orten gleichzeitig bzw. nirgends statt und lässt sich damit ebensowenig (geographisch) verorten wie Zeitungs-, Radio- oder TV-Kommunikation. ${ }^{5}$ Sendungen werden zwar irgendwo aufgezeichnet, doch die Ausstrahlung - und erst durch sie treten diese Medien in Aktion - ist nicht an den Ort der Aufzeichnung gebunden, sondern überwindet das Punktuelle des Ortes, indem sie sich über ein größeres oder kleineres Gebiet erstreckt. Die räumliche Entkopplung, die Kommunikationsmedien ermöglichen, bewirken die angesprochene, Ortlosigkeit ' in geographischer Hinsicht.

Nichtsdestotrotz trifft man überall auf Ortsangaben medialer Ereignisse: Man kann angeben, wo man etwas gelesen hat; es lässt sich in Erfahrung bringen, wo man ein bestimmtes Musikstück findet; auf die Frage, wo und wann welche Sendung zu sehen ist, hat sich sogar eine ganzen Zeitschriftensparte spezialisiert. Ohne die Verortung von Kommunikation scheint die Gesellschaft einfach nicht auszukommen (vgl. Stichweh 2000, S. 220ff.). Um sozial anschlussfähig zu sein, muss sie offenbar einem Ort zugerechnet werden können. Die Relevanz eines kommunikativen Ereignisses hängt erheblich davon ab, wie bekannt dessen Ort und Zeit sind. Je größer das Gebiet und der Zeitraum, in dem es zugänglich ist, desto größer auch die Chance, viele Menschen zu erreichen. Davon profitierte schon die Idee des Buches: Der Vorteil über große Entfernung transportabel und zeitlich beständig zu sein, fand im Buchdruck seine Ergänzung durch die Möglichkeit der Vervielfältigung. Manche Medien haben eine der beiden Seiten radikalisiert: Denkmäler setzen auf zeitliche Beständigkeit, verbleiben aber am Ort; Radiosender hingegen zielen auf ein großes räumliches Einzugsgebiet, ohne dass das Dargebotene zeitlich beständig wäre. Nur handelt es sich bei den meisten Medien bei der Ortsangabe nicht um eine geographische, stattdessen gibt man Stellen in Büchern, den Namen der Zeitung, den Fernsehkanal, den Titel der Musik-CD oder die Internetadresse an. Damit bewegen sich die Ortsangaben nicht in einem materiellen oder physischen, sondern in einem Kosmos der sozialen Verortung von Kommunikation. In diesem Kosmos sind Orte keine Punkte mit geographischer Länge und Breite, sondern geben an, wie medienvermittelte kommunikative Ereignisse zu erreichen sind. Das Internet bietet damit trotz seiner Virtualität selbst Orte der Kommunikation: nicht Köln, nicht Singapur, sondern www.chat.de. Daher ist weniger „die der Ortsgebundenheit allgemein zugesprochene Bedeutung fuir das Soziale zu relativieren“, wie Michael Paetau (1997, S. 106) meint; im Gegenteil: Der Ort scheint für soziale Operationen von unverminderter Wichtigkeit, nur die Bedeutung seiner geographischen Lage wird relativiert. Je bekannter es ist, wo ein kommunikatives Ereignis stattfindet, desto mehr Relevanz kann ihm zuteil werden.

Noch eine Radikalisienung von etwas, das vorher schon galt, bringt das Netz mit sich: Das Internet trägt mit dazu bei, Raum nicht mehr länger als gegebene

$5 \quad$ Streng genommen unterscheidet sie sich darin noch nicht einmal von einem Telefonat: Wo findet ein Gespräch statt? In Wien oder in Havanna oder etwa irgendwo dazwischen auf dem Mittelatlantischen Rücken? 
Konstante zu verstehen, als Behälter oder Rahmen, in dem sich Soziales abspielt, sondern als durch soziale Praktiken erst Erzeugtes aufzufassen und damit von Räumen auszugehen, die es nicht immer schon gibt, sondern die erst durch Handlungen und Kommunikation hervorgebracht werden (vgl. Schroer 2003, S. 13). Deshalb formuliert Paetau kurz und prägnant: „Kommunikation findet nicht in sozialen Räumen statt, sondern schafft diese Räume" (Paetau 1999, S. 279). Es ist also nicht die technische Infrastruktur des Internet, die die bunte soziale Welt des Cyberspace hervorzaubert; zweifelsohne bildet sie die technische Voraussetzung, doch ohne das kommunikative Getümmel bliebe sie sozial genauso bedeutungslos, wie ein Radiogerät ohne Empfang. Im Cyberspace surft man durch ein Meer von Kommunikationen, nicht durch ein Meer von Servern und Glasfaserkabeln. Schaltet man den Rechner aus, so bleibt die Infrastruktur zwar erhalten, der Cyberspace ist nun aber in weite Ferne gerückt.

Soziales findet stets an einem Ort statt: in der Küche, am Marktplatz, im Büro, am Lagerfeuer. Wie schon angesprochen, ist diese soziale Verortung jedoch nicht an physische Räume gebunden. Es waren die Verbreitungsmedien, die neue Bereiche der sozialen Entfaltung erschlossen; durch sie kamen neue, sozial konstitutierte Räume hinzu. Unser heutiges Leben ist maßgeblich durch medial vermittelte Kommunikation geprägt: Zeitungen, Bücher, Fernsehen und Radio sind aus unserem Alltag nicht wegzudenken. Dort entstehen Räume, die Platz für Soziales bieten, es aber auch formen. Da die meisten Menschen heute solchen Medien in einem erheblichen Umfang Zeit widmen, geht unser Erfahrungsraum weit über den des rein interaktiv Zugänglichen hinaus (vgl. Castells 2001, S. 381). Nicht nur Fiktion, wie im Roman oder in Kinofilmen, auch unsere Vorstellungen von der ,Wirklichkeit' sind dadurch geprägt: Was wir heute über Politik, Wirtschaft oder Wissenschaft wissen, wäre ohne die Verbreitung durch Medien undenkbar. Diese medialen Räume bieten jeweils eindeutig bestimmbare Orte für Kommunikation. Man kann angeben, wo man etwas gelesen hat - ob es am Schreibtisch oder in der Bahn war, spielt keine Rolle, in welcher Zeitung oder auf welcher Website, hingegen schon.

Entsprechend schaffen sich auch im Cyberspace Kommunikationen Räume und auch hier spielt es keine Rolle, von wo auf der Welt man eine Seite aufruft. Ausschlaggebend für die Verortung von Kommunikation ist die Adresse. Auch hier sind es soziale Operationen, die ein neues Reich sozialer Entfaltung entstehen lassen. „Die Gesellschaft bedient sich der Computernetzwerke, um Sozialität zu erzeugen“ (Paetau 1999, S. 272), und es ist diese spezifische Sozialität, die es näher zu beleuchten gilt, wenn man nach Veränderungen sucht, die nicht schon von anderen Kommunikationsmedien her hinlänglich bekannt sind. Der Cyberspace bietet also dem sozialen Treiben neuen Raum. Allerdings ist nicht nur dieser neue Raum unvertraut, sondern dort treten auch zuvor unvertraute Formen von Sozialität auf, wie in den beiden folgenden Abschnitten gezeigt werden soll. 


\section{DER CYBERSPACE ALS FRAGMENTIERTE ÖFFENTLICHKEIT}

Ähnlich wie das Telefon ermöglicht auch das Internet verschiedenen Personen, miteinander in Kontakt zu treten, sich zu unterhalten, zu quatschen, zu, chatten'. $\mathrm{Ob}$ per Email, Internet Relay Chat (IRC) oder Diskussionsforum, das Netz bietet viele Möglichkeiten für ein Gespräch über große Entfernungen hinweg. Dabei können sich wie beim Telefon zwei (aber auch mehr) Personen ungestört und abgeschottet von anderen austauschen. Daneben erlaubt es das Web allerdings auch, Diskussionen, Unterhaltungen oder Beratungen in aller Öffentlichkeit zu führen, und geht darin über fernmündliche Gespräche oder den Briefverkehr hinaus. Folglich tummeln sich dort alle nur denkbaren Mitteilungen; jeder kann sich in die öffentlichen Gespräche einbringen oder zumindest als Publikum teilnehmen. Es geht zu wie in einem großen Wirtshaus: Es gibt kein Thema, das es nicht gibt; an jedem Tisch wird über etwas anderes gesprochen; man könnte überall lauschen und sich fast überall einmischen; und ebenso wie am Stammtisch verfügt so manches Internetforum über Mechanismen des ,Mundtotmachens', sollte eine Person ungeliebte Äußerungen von sich geben. In diesem Fall kann man sich dann immer noch an einen anderen Tisch setzen - und sei es alleine.

Dass das Netz durch die Abwesenheit der Körper andere Wege zur Präsentation des Selbst gestattet, ist mittlerweile hinlänglich bekannt (vgl. Höflich 1998; Walther 1996, S. 17ff.). Das führt allerdings nicht nur zu den breit diskutierten Optionen in der Darstellung der eigenen Persönlichkeit, sondern auch zu der unübersichtlichen Struktur dieses, virtuellen Wirtshauses': Um den Cyberspace zu betreten, braucht man seinen Körper nicht von der Stelle zu bewegen; entsprechend riesig ist die Zahl der Gäste. Die oft angesprochene These von Marshall McLuhan, dass die „Welt nur mehr ein Dorf" (McLuhan 1995, S. 17) sei, mag angesichts dieser Dimension falsche Assoziationen wecken. $\mathrm{Zu}$ sehr denkt man bei Dorf an Ordnung, Übersichtlichkeit, soziale Kontrolle und Kleinräumigkeit. Zwar könnte man sagen, durch die Relativierung der Entfernungen, an denen das Internet wesentlichen Anteil hat, sei die Welt sozusagen ,kleiner' geworden, der Cyberspace wuchs dafür aber explosionsartig an. So sehr die Relevanz der Entfernung für Kommunikation geschrumpft ist, so sehr ist der Informations-Dschungel ,ins Kraut geschossen'. Mit dem Internet entstand weniger ein globales Dorf als vielmehr ein ,globales Wirtshaus': Man geht hin, wann man will, trifft sich mit anderen oder ,konsumiert' nur, verzehrt die bereitgestellten Speisen und schaut den anderen Gästen zu. Anders als in einer Wirtschaft ist nur, dass man zwar von manchen Tischen und aus so manchen Sälen verbannt werden kann, vor einem völligen Hinauswurf bleibt man jedoch verschont (außer man bezahlt die Telefonrechnung nicht).

Während übliche Wirtshäuser in Theorien zur Struktur der modernen Öffentlichkeit nur eine marginale Rolle spielen, verhält sich das beim globalen Wirtshaus anders: Immer wieder wird ihm vor allem eine demokratisierende Tendenz zugesprochen (vgl. Rheingold 1995; Krotz 1998, S. 122ff.; Roesler 1997). Das Internet wird als willkommene Gegenkraft gegen die etablierten unidirektionalen Massenmedien begrüßt. Viele erhoffen sich eine Rückkehr zu einer weniger ein- 
seitig dominierten Öffentlichkeit, bei der die Bürger endlich wieder etwas zu sagen haben.

Das liegt nicht zuletzt daran, dass unsere Vorstellung von Öffentlichkeit nach wie vor die griechische Agora als Idealbild vor Augen hat. Noch heute gilt die Versammlung einer Gemeinschaft, die kollektiv Beratungen abhält, als Idealbild öffentlicher Meinungsbildung. Das schlägt sich auch in Jürgen Habermas ‘ bis heute maßgeblicher Studie über den Strukturwandel der Öffentlichkeit nieder (Habermas 1990), die sich mit den Bedingungen der Möglichkeit eines öffentlichen Räsonnements in einer Gesellschaft beschäftigt, in der sich die Bürger nicht mehr zu Beratungen an einem Ort versammeln können. Die Grundidee der Gleichheit aller Beteiligten, der Zugänglichkeit für jeden und des dialogischen Charakters ist erhalten geblieben. Dieses bis heute nachwirkende Ideal des AgoraModells zeichnet sich durch unmittelbare Einwirkungsmöglichkeit aus, ein Publikum im Sinne einer zuschauenden, passiven Masse gibt es nicht. Die Beratungen folgen eher dem Schema eines Plenums: Jeder hat die Möglichkeit, das Wort zu ergreifen und damit von der Rolle des Zuschauers zu der eines Akteurs zu wechseln. Öffentlichkeit erzeugt die Agora aber nicht nur durch freie Zugänglichkeit, sondern in hohem Maße auch dadurch, dass sie einen gemeinsamen Bezugsrahmen bereitstellt. Dort werden gemeinsame Probleme beraten, gemeinsame Themen behandelt und für alle gemeinsam verbindliche Entscheidungen gefällt. Sie konstituiert eine öffentliche Referenz, in der das Gemeinsame konvergiert und auf die man sich bezieht, wenn man die Gemeinschaft im Blick hat. Um es pointiert zu formulieren: Sie konstituiert Gemeinschaft.

Verglichen mit dem Ideal einer Agora haben die modernen drucktechnischen und elektro-magnetischen Massenmedien zu einer oft geschmähten Einseitigkeit der Kommunikationsströme geführt. Zwar ist weiterhin niemandem prinzipiell verwehrt, sich öffentlich zu äußern, allerdings bewirken die dominierenden Verbreitungsmedien eine Asymmetrie, die manchen zu einer überwältigenden Medienpräsenz gegenüber der breiten Masse verhilft. Damit kristallisierte sich mit den Massenmedien ein rein rezipierendes Publikum heraus, welches eigentlich nicht mehr einen Teil der Öffentlichkeit im engeren Sinne bildet - es handelt sich dann nur noch um Zuschauer. Daraus kann gefolgert werden, dass in dem Maße, in dem Zugangsbarrieren zur massenmedialen Öffentlichkeit bestehen, sich ihre Bedeutung als gemeinsamer Bezugsrahmen vertieft (vgl. Wehner 1997a). Indem die Präsenz als Notwendigkeit für eine Rezeption aufgehoben wurde, konnte der Umfang des Publikums enorm vergrößert werden. Die Massenmedien erfüllen damit die gleiche Funktion wie eine Agora: Sie dienen als gemeinsame Referenz und bilden, obwohl von einer traditionellen Gemeinschaft keine Rede sein kann, eine Gemeinschaft heraus; allerdings handelt es sich dabei nicht um eine Gemeinschaft gemeinsam beratender Bürger, sondern um eine Gemeinschaft von Rezipienten. $^{6}$ Massenmedien gewährleisten einen gemeinsamen Themenhorizont,

$6 \quad$ Zur Nation als vorgestellte Gemeinschaft und zur Rolle der Massenmedien bei der Konstitution derselben: siehe Anderson 1996; zusammenfassend zur sozialen Konstitution von Nationen und dem gängigen Mythos von deren Ursprünglichkeit: Kößler 1995. 
gemeinsame Wissensbestände und auch eine gemeinsame Aktualität.

Abgesehen von den notwendigen technischen Voraussetzungen, die vor allem im globalen Maßstab äußerst ungleich verteilt sind, zeichnet sich das Internet durch freie Zugänglichkeit aus und reaktiviert insofern Eigenschaften des AgoraModells: Es gestattet sogar, und darauf beruht ein Teil der Euphorie, die das Medium begleitet, jedem die Möglichkeit, sich einzubringen; prinzipiell könnte sich jeder am netzöffentlichen Diskurs beteiligen. Allerdings herrschen andere Voraussetzungen: Das Agora-Modell beruht gerade auf der Überschaubarkeit der Teilnehmerzahl und einer gleichzeitigen Fokussierung des Diskursstroms. Es kann nicht jeder gleichzeitig reden; wogegen genau das im Cyberspace möglich und üblich ist. Es gibt kein gemeinsames Forum, sondern es gliedert sich in unzählige Foren, die meist nichts voneinander wissen und nichts als die Benutzung des selben Mediums miteinander teilen. Es herrschen eben Zustände wie im Wirtshaus und nicht wie bei einer Bürgerversammlung. Die Gemeinsamkeit, die eine Agora oder die Massenmedien hervorbringen, entsteht im Netz nicht. Zwar wird das Netz von manchen Beobachtern allein aufgrund seiner Verbreitung als Massenmedium eingestuft (vgl. Morris/Ogan 1996), eine wesentliche Eigenschaft kommt ihm jedoch nicht zu: Es bringt keine gemeinsame „Hintergrundrealität“ (Luhmann 1996, S. 173) hervor, auf die man sich im Vertrauen darauf beziehen kann, dass sie auch anderen bekannt sei.

Dagegen könnte man einwenden, dass die Funktionsbestimmung von Massenmedien, wie sie im Rahmen der soziologischen Systemtheorie geäußert wird (vgl. Luhmann 1996, S. 169ff.; Esposito 1995, S. 235ff.; Wehner 1997a, S. 100ff.), schon durch das dutzendfache Angebot an Fernsehkanälen in Frage gestellt wird. Schließlich sind in den letzten Jahrzehnten immer mehr Auswahlmöglichkeiten hinzugetreten, so dass von einer Bündelung der Publikumsaufmerksamkeit auf wenige staatliche Sender keine Rede mehr sein kann. Dieses Argument müsste man allerdings noch erweitern: Schon lange vor Einführung des Privatfernsehens und der explosionsartigen Angebotszunahme gab es im Bereich der Massenmedien eine große Vielfalt. Gerade auf dem Zeitungs- und Zeitschriftenmarkt tummelt sich schon seit geraumer Zeit ein bunter und unübersichtlicher Blätterwald. Die Systemtheorie behauptet also, dass trotz dieser Vielfalt die Funktion der Massenmedien darin liege, einen gemeinsamen "thematischen Horizont" (Wehner 1997a, S. 101) bereitzustellen. Es werde ein gemeinsamer massenmedial erzeugter Bezugsrahmen hergestellt, den man als bekannt voraussetzen und an den man auch bei Unbekanntheit des Gegenübers anschließen kann. In der Tat fällt auf, dass trotz der bunten Vielfalt an Zeitungen und Sendern sich doch in den Massenmedien herauskristallisiert, welche Themen als relevant angesehen werden. Es gibt zwar Unterschiede, aber es gibt offenbar Themen, an denen niemand vorbeikommt. Auch mit diesen kann verschieden umgegangen werden, aber darüber hinweggehen kann keine Redaktion. Vom Bürgerkrieg im Kongo war bis vor kurzem kaum etwas zu hören, über die Auseinandersetzungen zwischen Israelis und Palästinensern wird dagegen seit Jahrzehnten beinahe täglich berichtet. In Zeiten von Fussballweltmeisterschaften ist es kaum möglich, sich ihnen zu entziehen; das gilt auch für Sender, die keine Liveübertragung anbieten. Manche 
Kinofilme werden zum öffentlichen Thema (und auch das wiederum vermittelt durch Massenmedien), während andere in der Versenkung verschwinden. Und man kann zwar nicht unbedingt sein Werk als bekannt voraussetzen, aber zumindest erwarten, dass der Name Shakespeare gewisse Assoziationen weckt. ${ }^{7}$ Diese Themenfokussierung löst sich im Cyberspace endgültig auf: Der Themenauswahl sind keine Grenzen mehr gesetzt und diese Offenheit wird weidlich genutzt. Hier verschwimmen auch die Grenzen von öffentlich und privat; private Themen werden öffentlich behandelt und öffentliche privat. Einen gemeinsamen thematischen Bezugsrahmen bringt das Netz nicht hervor. Unterhält man sich mit einer unbekannten Person auf der Straße, so kann man nicht wissen, mit welchen Websites sie vertraut ist und mit welchen nicht.

Aber macht das wirklich den Unterschied zu den klassischen Massenmedien aus? Lassen diese sich denn tatsächlich auf ihre Funktion, eine gemeinsame ,Realität ' herzustellen, reduzieren? Sicherlich gilt Niklas Luhmanns bekannte Formulierung: „Was wir über unsere Gesellschaft, ja über die Welt, in der wir leben, wissen, wissen wir durch die Massenmedien" (Luhmann 1996, S. 9). Es gilt jedoch auch: Nicht alles, was in den Massenmedien berichtet wird, weiß man. Man kann nicht alle Zeitungen lesen und nicht alle Sender gleichzeitig sehen bzw. hören. Die Realität der Massenmedien umfasst offenbar wesentlich mehr als das, was in Interaktionen unter Anwesenden als Hintergrundrealität vorausgesetzt werden kann. Systemtheoretisch liegt die Antwort darin, dass ähnlich wie im politischen System, in dem nicht alle Operationen an der Funktion, Kapazitäten für kollektiv bindende Entscheidungen bereitzuhalten, unmittelbar beteiligt sind, auch nicht alles im Bereich der Massenmedien zur Erzeugung einer gemeinsamen Realität beiträgt. Stets aber findet, auf die eine oder andere Weise vermittelt, eine Orientierung an der Funktion statt. Das angesprochene Publikum muss dabei keineswegs mit der Bevölkerung eines Nationalstaats zusammenfallen. Wie sich gerade an den zahllosen, auf eng umgrenzte Themen spezialisierten Zeitschriften zeigt, hat man es mit einem ganzen Geflecht unterschiedlicher Publika zu tun.

Zweifellos gibt es auch im Cyberspace Websites, die sich an der Funktion der Massenmedien orientieren. Gerade Zeitungen bieten im Netz beinahe Kopien ihrer Papierausgaben. Das Internet schließt also massenmediale Arrangements nicht aus, diese können aber auch nicht als charakteristisch bezeichnet werden. Vieles orientiert sich nicht an der Funktion der Massenmedien, sondern folgt der Logik anderer Funktionssysteme, wie Wirtschaft oder Wissenschaft, oder muss ganz anders eingeordnet werden. In seiner Vielfalt ließe sich der Cyberspace mit dem Büchermarkt vergleichen: Manches ist bekannt, anderes nicht, und zu jedem Thema ist etwas zu finden. Allerdings orientiert sich der Büchermarkt schon aufgrund des Verlagswesens eher an massenmedialen Erwägungen: Man versucht Leser zu erreichen. Das Netz bietet demgegenüber andere Möglichkeiten. Es erlaubt eine stärkere Entkopplung von der Nachfrage und den Gewohnheiten des

7 Ein eindrucksvolles Beispiel fur die Fokussierung auf gewisse Themen ist sicherlich auch, dass selbst Bücher von Dieter Bohlen nicht einfach ignoriert werden, wie sie es sicherlich verdient hätten, sondern selbst in seriösen Zeitungen thematisierbar sind, und sei es nur, um Spott und Hohn auszugießen. 
Publikums. Die skurrilen Websites, wovon es einige zu bewundern gibt, entstehen gerade dadurch, dass man weniger darauf angewiesen ist, sich am Publikum zu orientieren. Auch kennt der Cyberspace keine internen Exklusionsmechanismen: Wenn die technischen und finanziellen Voraussetzungen vorhanden sind, steht einem Internet-Auftritt nichts mehr im Wege; es gibt keine Verlage, die als Filter wirken; man muss nichts Druckreifes parat haben und nicht stilistisch angemessen schreiben können. Auch in anderer Hinsicht greift ein Vergleich mit der Welt der Bücher zu kurz, denn Interaktivität bedeutet mitunter mehr als nur das Springen von Hypertext zu Hypertext, was sich vor allem die Wirtschaft zu Nutze gemacht hat. Während Bücher allenfalls Texte über das wirtschaftliche Geschehen enthalten, vollziehen sich im Cyberspace Operationen des Wirtschaftssystems: Preise werden ausgehandelt, Geldforderungen erhoben, Zahlungen getätigt. Das Web lässt sich also keineswegs einfach unter die Massenmedien subsumieren, noch trägt es zur Erzeugung einer gemeinsamen Hintergrundrealität bei.

Doch nicht nur aus einer funktionalistischen Perspektive ist Skepsis gegenüber allzu großen Erwartungen an die Internet-Technologie und ihre Auswirkungen auf die öffentliche Meinungsbildung angebracht. „Bürgerliche Öffentlichkeit", schreibt Habermas, „läßt sich vorerst als die Sphäre der zum Publikum versammelten Privatleute begreifen" (Habermas 1990, S. 86). Für seinen diskurstheoretischen Ansatz müsste das Web eigentlich in eine vielversprechende Richtung weisen, jedoch folgt der Diskurs dort allem Anschein nach keinerlei Rationalität und es findet auch kaum das damit verbundene „öffentliche Räsonnement“ (ebd.) statt. Im Cyberspace erfolgt keinerlei Integration der Perspektiven durch den Austausch von Argumenten, auch fehlt ein übergreifender kritischer Diskurs. Durch die beinahe freie Zugänglichkeit für jedermann fehlt dem Netz jegliches kompetitive Moment, das einen gewissen Zwang, und sei es nur der Zwang des besseren Arguments, ausüben könnte. Das Internet fügt sich nicht der auf Gemeinsamkeit bezogenen Vorstellung von Öffentlichkeit, sondern reduziert diese auf freie Zugänglichkeit und die Möglichkeit für jeden, sich für andere zumindest potentiell wahrnehmbar zu äußern. Da die Rezeption keine Bündelung erfährt und somit jeder sich einem anderen Diskursfeld gegenüber sieht und auf anderes bezieht, ist die zwangsläufige Folge eine gesteigerte Fragmentierung der Öffentlichkeit. Der Cyberspace bildet keine einheitliche allen gemeinsame Öffentlichkeit, sondern ein aus allen Nähten platzendes, unüberschaubares öffentliches Geschehen. Der gebündelten Öffentlichkeit der Massenmedien tritt der fragmentierte Kommunikationsraum des Cyberspace gegenüber. ${ }^{8}$

Angesichts aktueller Trends können allerdings nur noch Teile des WWW überhaupt als öffentlich charakterisiert werden, denn es greifen immer stärker „Cyber-Segmentierungen" um sich, wie Saskia Sassen (1997) sie beschrieben hat. Unternehmen und andere Organisationen nutzen die Infrastruktur des Internets, um sich dort eigene Sites einzurichten, zu denen nur noch autorisierte Personen Zugang haben. Aber auch eine zunehmende Kommerzialisierung ist festzustellen,

8 Deshalb wird das Internet auch langfristig andere Medien nicht verdrängen, sondern lediglich ergänzen, wie auch Josef Wehner (1997a) vermutet. 
so dass so manches ausschließlich gegen Entrichtung von Gebühren offen steht. Solche mit Ausschlussmechanismen versehenen Bereiche im Netz sind nicht öffentlich in dem Sinne, dass die prinzipielle Zugänglichkeit für jedermann aufgehoben ist, indem User aufgrund eigener Selektionskriterien wie Firmenzugehörigkeit oder Zahlungsfähigkeit ausgeschlossen werden. Sicherlich ist auch der generelle Zugang zum Internet in der Regel nicht umsonst zu haben, doch im Falle von Intranetzen oder kommerziellen Online-Diensten verfügen Wächter über Ein- oder Ausschluss und können so selbst willkürlich die Bedingungen für den Zugang diktieren. Während also der öffentliche Bereich des Cyberspace als fragmentiert aufgefasst werden muss, weil kein gebündelter Diskurs auszumachen ist, richten sich daneben immer mehr nicht-öffentliche Bereiche ein und führen zu Segmentierungen.

\section{SOZIALES OHNE SOCIUS}

Mit der angesprochenen Fragmentierung geht zwangsläufig auch eine radikalisierte Individualisierung der Perspektiven einher. Aus dem scheinbar unendlichen Universum des Cyberspace selektiert jeder für sich Informationen. Die „Selektionslast", wie Josef Wehner (1997b, S. 140) es formuliert, liegt auf der Nutzerseite. Sorgen Massenmedien nach wie vor dafür, dass jeder zumindest dort ein mehr oder weniger gleiches Gesicht der Welt präsentiert bekommt, so verhindert das Netz eine solche Kongruenz der Perspektiven geradezu. Doch ermöglicht das nicht nur einen individualisierten Zugang zu den bereitgehaltenen Informationen, zugleich stellt der Cyberspace auch individualisierte Räume zur Verfügung. $\mathrm{Zu}$ nehmend trifft man auf persönlich zugeschnittene Seiten und Angebote. Versandhäuser halten für jeden Kunden spezifisch gestaltete Seiten wie etwa Warenkörbe oder speziell zugeschnittene Angebote bereit, auf virtuellen Friedhöfen lassen sich Seiten einrichten, die nur einem bestimmten Personenkreis oder auch nur einer Person offen stehen (vgl. Schwibbe/Spieker 1999) usw. „Während die Medien heute tatsächlich global miteinander verknüpft sind und während Sendungen und Botschaften durch das globale Netzwerk zirkulieren, leben wir nicht in einem globalen Dorf, sondern in individuell zugeschnittenen Hütten, die global produziert und lokal verteilt werden" (Castells 2001, S. 390).

Der Cyberspace fungiert damit nicht nur als Medium, über das Menschen miteinander in Kontakt treten und sich austauschen können, sondern er bietet sich selbst zugleich als Partner an, mit dem man interagieren kann. Wie immer wieder betont wird, zeichnen sich neue Medien eben durch ,Interaktivität' aus, was nichts anderes bedeutet, als dass Maschinen in Interaktion mit Menschen treten (vgl. Esposito 1995; Dollhausen 2000, S. 118 f.). In Anlehnung an die von Elena Esposito (1993) geprägte Formel vom „Computer als Medium und Maschine“ könnte man in unserem Fall also vom, Cyberspace als Medium und Interaktionspartner* sprechen. Sicherlich gilt das in einem eingeschränkten Sinne schon für viele Fahrscheinautomaten und mehr noch für Computerspiele, im Web allerdings weiß man nicht mehr, mit wem oder was man gerade interagiert. Es lässt sich im $\mathrm{Cy}$ - 
berspace oftmals gar nicht erkennen, ob überhaupt bzw. wie viele Menschen an einer Interaktion beteiligt sind. Die Grenzen verschwimmen. Wenn sich Interaktion mit dem Cyberspace aber nicht mehr von einer mit Menschen unterscheiden lässt, wenn also Interaktion nicht mehr davon abhängt, dass es sich beim Interaktionspartner um einen Menschen handelt, so steht auch in Frage, dass Soziales notwendig auf einer zwischenmenschlichen Ebene beruht. Dadurch wird Soziales ohne socius, also ohne ein menschliches Gegenüber, denkbar.

Die prinzipiell denkbare und auch hie und da anzutreffende Ununterscheidbarkeit von Mensch/Mensch- und Mensch/Maschine-Interaktionen macht skeptisch gegenüber der Prämisse, Menschen bildeten die Grundbausteine sozialer Systeme. Im Anschluss an Luhmann wird innerhalb der Soziologie schon seit einiger Zeit damit experimentiert, Kommunikation (und nicht Handlung) als basale soziale Operation zu begreifen. Soziales hat dann seinen Ort nicht mehr dort, wo Menschen sind, sondern dort, wo Kommunikation stattfindet (vgl. Luhmann 1984, S. $191 \mathrm{ff}$.; Luhmann 1995, S. 113ff.). Wenn man nicht mehr sagen kann, wer oder was mit wem oder was gerade kommuniziert, wie das im Cyberspace der Fall ist, drängt sich eine solche Perspektive geradezu auf, denn immerhin lässt sich feststellen, dass kommuniziert wird. ${ }^{9}$

Peter Fuchs knüpft an Luhmanns Theorie sozialer Systeme an und spricht davon, dass wir es beim Netz mit einem sozialen System zu tun haben. Das Internet verfüge über eine „Eigensensibilität“, mit der es ,,in sich selbst auf sich selbst reagieren" (Fuchs 1998, S. 303) könne. Die Quelle für eine solche Eigensensibilität stellt ihm zufolge eine eigene Operationsweise dar, mit der das Web Sinn prozessiert: Als elementare Einheit des Systems sieht er „die Operation [...] des linkens" (ebd.). Das Internet als soziales System experimentiere damit, wieviel Bewusstsein der Kommunikation entzogen werden kann, so dass sich ,hyperautonome Formen der Kommunikation" (Fuchs 1998, S. 310) beobachten ließen.

Für Fuchs stellt das WWW also ein operativ geschlossenes System dar, dessen Autopoiesis ,eine spezifische Operation" (Fuchs 1998, S. 308), die des Linkens, zugrunde liegt. Dem ist allerdings entgegen zu halten, dass zu einem sozialen System mehr gehört als ein exklusiver Operationsmodus. Wenn man wie Fuchs durchgängig auf der Grundlage von Luhmanns Systemtheorie argumentiert, so bilden Kommunikationen die basalen Elemente sozialer Systeme, wobei darunter die dreifache Selektion von Mitteilung, Information und Verstehen gefasst wird. Dementsprechend fasst auch Fuchs die Operation des Linkens als Kommunikation auf: Als Mitteilungen fungierten demnach die eingestreuten Durchschaltofferten, die Links, die auf einer Website anzutreffen sind; die mitgeteilte Information ergebe sich daraus, ,daß man und wohin man durchschalten kann“ (Fuchs 1998, S. 305); das Durchschalten, die Operation des Linkens selbst bilde die Verstehenskomponente. Der Kommunikationsstrom kommt folglich dadurch zustande, dass immer weitergeklickt, ein Link nach dem anderen aktiviert wird. Er ist damit nichts anderes als das ,Surfen' durch den Cyberspace. Hier wird nun

9 Auch wenn mancher Beobachter solche Kommunikation als ,asozial " bewerten würde, so macht sie heute doch unbestreitbar einen nicht unbedeutenden Teil des sozialen Lebens aus. 
deutlich, inwiefern bei Fuchs eine Verwechslung der Ebenen vorliegt: Nicht das am Bildschirm sichtbare Web selbst bildet ein soziales System, wie er behauptet, sondern die Interaktion eines Benutzers mit diesem. Eine vergleichbare Situation ist die eines Autofahrers, der sich durch den berüchtigten Schilderwald kämpft: Er bahnt sich seinen Weg durch das Straßennetz; er leistet den auf den Schildern dargestellten Anweisungen zum Teil Folge, zum Teil aber auch nicht; er sucht sich seinen Weg, wobei er sich an den Hinweisschildern orientiert, die den nächsten Ort oder die Bezeichnung der Straße angeben; an manchen Kreuzungen biegt er ab, an anderen fährt er geradeaus. Wie beim Netz auch, so bildet nicht der sichtbare Schilderwald den Kommunikationsstrom, sondern selbstverständlich die Reise durch ihn hindurch.

Außerdem spricht Fuchs eine Besonderheit sozialer Systeme an, indem er darauf hinweist, dass sie nicht ohne strukturelle Interpenetration mit psychischen Systemen auskommen (Fuchs 1998, S. 311 ff.), eine andere wichtige Besonderheit findet dagegen nicht einmal eine Erwähnung: Soziale Systeme emergieren in Situationen doppelter Kontingenz (vgl. Luhmann 1984, S. 148ff.). Aus einer solchen Situation entsteht die notwendige Dynamik, die soziale Systeme vorwärts treibt. Wie Fuchs selbst zu Beginn seines Aufsatzes feststellt, ist das Netz zunächst nichts weiter als ein Register, in das Dokumente eingehängt werden (Fuchs 1998, S. 303). Dieses Register für sich allein operiert allerdings nicht, sondern verhält sich so reglos wie ein Archiv. Was dem Cyberspace Leben verleiht, ist die Interaktion mit einem User. ${ }^{10}$ Beim ,Surfen ${ }^{\circ}$ tritt dieser mit dem Cyberspace in eine Situation doppelter Kontingenz, wodurch ein neues, ein soziales System emergiert. Wenn man den Blick auf das Verhältnis von User und Cyberspace richtet, so wird deutlich, dass sie wechselseitig füreinander ,black boxes' bleiben; sie lassen sich in ihrer Selbstbestimmung letztlich nicht determinieren. Erst ihre Interaktion lässt ein System emergieren, das eine gegenseitige Orientierung aneinander erfordert und damit mehr ist als ein schlichtes Register. Es entsteht gewissermaßen Sozialität ohne Geselligkeit. Diese Form der Sozialität bedarf keiner Zimmer, keiner Stühle, keiner Tische, keiner Nähe und keiner menschlichen Interaktionspartner. Aber sie bedarf eines Ortes - und der ist virtuell.

Insgesamt sieht sich damit der Nutzer dem Netz gegenüber in einer vergleichbaren Situation wie gegenüber einem menschlichen Interaktionspartner: Ebenso wie für ihn das andere Bewusstsein generell unzugänglich bleibt, ist es ihm auch unmöglich, das Netz zu durchschauen. Stets bekommt er nur aktuelle Formen zu Gesicht, doch das Netz selbst entzieht sich seinem Zugriff. Man weiß nicht, was sich hinter dem aktuell Sichtbaren verbirgt und man weiß nicht, was einem in der Vergangenheit alles verborgen geblieben ist. Nur geschieht das hier nicht durch die unergründliche Tiefe des Bewußtseins, sondern durch die unergründliche Weite des Cyberspace.

10 Von kaum abzuschätzender Bedeutung für die zukünftige Entwicklung ist, dass durch die zunehmende Verbreitung von sogenannten ,agents' nicht mehr nur menschliche Individuen als User denkbar sind und somit vielleicht auch irgendwann Situationen doppelter Kontingenz ohne menschliche Beteiligung entstehen könnten (zu den Agenten aus soziologischer Perspektive vgl. Rammert 1998). 
Von hier aus kann man Blick auf den Rahmen lenken, in dem sich Soziales im Netz abspielt: Betrachtet man allein das WWW, so zeigt sich dort eine radikale Beschränkung des Kommunizierbaren. Zumeist werden die Alternativen des Benutzers darauf reduziert, dass er Verschiedenes anklicken kann, woraufhin er auf andere Seiten gelangt, die ihm wiederum nur verschiedene Möglichkeiten des Durchschaltens gewähren. Die viel gerühmte Interaktivität reduziert sich hier aufs Klicken. Die zunächst unüberschaubare Kontingenz möglicher Anschlüsse an das Kommunikationsangebot, das Websites bereitstellen, wird darauf beschränkt, den einen oder anderen Hyperlink zu betätigen. Und selbst wenn Eingaben über Tastatur möglich sind, so hat man sich zumeist einer vorgegebenen Schablone zu fügen oder man muss damit rechnen, dass man erneut zur Eingabe, diesmal aber innerhalb des vorgegebenen Rahmens, aufgefordert wird. Es werden in der Regel nur wenige Optionen gelassen, die vom Netz akzeptiert werden.

Diese schablonenhafte Anlage des Cyberspace bewirkt zweierlei: Erstens führt sie zu einer radikalen Reduktion von Kontingenz. Das müssen zwar auch andere soziale Systeme leisten, doch die Alternative, einen von verschiedenen Links anzuklicken oder abzuschalten, beschränkt den Möglichkeitsraum auf ein Minimum. Die Welt jenseits des Cyberspace bietet stets mehr Vielfalt und schließt abwegige Möglichkeiten nicht einfach technisch aus. Prinzipiell ist alles sagbar, man muss dann allerdings mit Konsequenzen rechnen; im Web hingegen ist schon die Sagbarkeit radikal beschnitten.

Zweitens ermöglicht erst diese schablonenhafte Kommunikation, dass nicht mehr unterscheidbar ist, mit wem oder was man interagiert. Maschinen sind (noch?) nicht zur Beteiligung an komplexeren Kommunikationsverläufen fähig und Menschen müssen sich, wollen sie das Netz nutzen, in dessen grobes Raster fügen. Durch den standardisierenden Filter des WWW geschickt, wird der Entstehungskontext von Mitteilungen ununterscheidbar.

Immer wieder wird erwähnt, dass der Cyberspace durch die rasante Entwicklung unüberschaubare Ausmaße angenommen hat: Die Zahl der Sites übersteigt längst jedes Vorstellungsvermögen und zudem findet ein kontinuierlicher Wandel statt. Obwohl auf der konkreten Seite, wie eben angesprochen, stets nur ein begrenzter Spielraum an Möglichkeiten besteht, scheinen sie bezogen auf den Cyberspace insgesamt unbegrenzt. Trotzdem lässt sich das Web eher als geronnener Verweisungshorizont denn als Raum der unbegrenzten Möglichkeiten beschreiben: Das, worauf verwiesen wird, muss stets schon vorhanden sein, um es abrufen zu können; Websites müssen erst ins Netz gestellt werden, ehe sie ,verlinkt" werden können. Bei face-to-face-Kontakten können hingegen Verweisungen ad hoc auftauchen; das, worauf verwiesen wird, geht nicht selten erst aus dem Verweis hervor. Auch auf seine Gesamtheit bezogen zeigt das Web also eine schablonenhafte Gestalt, auch wenn man stets nur einen winzigen Teil davon zu Gesicht bekommt und geblendet durch die Vielfalt beinahe vergisst, wie starr das Raster des Netzes eigentlich ist.

Abschließend kommen wir noch einmal kurz zurück zur Symbolkraft der Bezeichnung ,Cyberspace‘: Der Cyberspace bietet einerseits interpersonaler Kommunikation einen Raum und beherbergt damit soziale Operationen in einer frag- 
mentierten Öffentlichkeit. Zugleich tritt der Cyberspace aber auch selbst als Kommunikationspartner auf. Er ist nicht nur selbst ein Raum, der aus sozialen Operationen entstanden ist, sondern auch Interaktion mit ihm lässt einen Raum besonderer Sozialität entstehen - eine Sozialität, für die es keine Rolle spielt, wer oder was als Interaktionspartner fungiert, und die somit auf den menschlichen socius verzichten kann.

\section{LITERATUR}

Anderson, Benedict (1996): Die Erfindung der Nation. Zur Karriere eines folgenreichen Konzepts, Berlin.

Beck, Ulrich (1997): Was ist Globalisierung? Intümer des Globalismus - Antworten auf Globalisierung, Frankfurt a.M.

Beck, Ulrich (1998): Wie wird Demokratie im Zeitalter der Globalisierung möglich? - Eine Einleitung, in: ders. (Hrsg.): Politik der Globalisierung, Frankfurt a.M., S. 7-66.

Bollmann, Stefan (1995): Einführung in den Cyberspace, in: ders. (Hrsg.): Kursbuch Neue Medien. Trends in Wirtschaft und Politik, Wissenschaft und Kultur, Mannheim, S. 163-165.

Castells, Manuel (2001): Das Informationszeitalter. Teil 1: Der Aufstieg der Netzwerkgesellschaft, Opladen.

Dollhausen, Karin (2000): Neue Medien und Kommunikation: Medien- und kommunikationstheoretische Überlegungen zu einem Forschungsfeld, in: Voß, G. Günter u.a. (Hrsg.): Neue Medien im Alltag. Begriffsbestimmungen eines interdisziplinären Forschungsfeldes, Opladen, S. 107-126.

Esposito, Elena (1993): Der Computer als Medium und Maschine, in: Zeitschrift für Soziologie, Jg. 22, S. 338-354.

Esposito, Elena (1995): Interaktion, Interaktivität und die Personalisierung der Massenmedien, in: Soziale Systeme, Heft 2, S. 225-260.

Flusser, Vilém (1995): Verbündelung oder Vernetzung? in: Bollmann, Stefan (Hrsg.): Kursbuch Neue Medien. Trends in Wirtschaft und Politik, Wissenschaft und Kultur, Mannheim, S. $15-23$.

Fuchs, Peter (1998): Realität der Virtualität - Aufklärungen zur Mystik des Netzes, in: Brill, Andreas und Michael de Vries (Hrsg.): Virtuelle Wirtschaft. Virtuelle Unternehmen, virtuelle Produkte, virtuelles Geld und virtuelle Kommunikation, Opladen, S. 301-322.

Gibson, William (1987): Neuromancer, München.

Habermas, Jürgen (1990): Strukturwandel der Öffentlichkeit. Untersuchungen zu einer Kategorie der bürgerlichen Gesellschaft, Frankfurt a.M.

Höflich, Joachim R. (1998): Computerrahmen und Kommunikation, in: Prommer, Elizabeth und Gerhard Vowe (Hrsg.): Computervermittelte Kommunikation. Öffentlichkeit im Wandel, Konstanz, S. 141-174,

Kößler, Reinhart (1995): Nation und Nationalismus, in: Kneer, Georg u.a. (Hrsg.): Soziologie. Zugänge zur Gesellschaft, Bd. 2, Spezielle Soziologien, Münster, S. 137-149.

Krotz, Friedrich (1998): Digitalisierte Medienkommunikation: Veränderungen interpersonaler und öffentlicher Kommunikation, in: Neverla, Irene (Hrsg.): Das Netz-Medium. Kommunikationswissenschaftliche Aspekte eines Mediums in Entwicklung, Opladen, S. 113-136. 
McLuhan, Marshall (1995): Die magischen Kanäle. Understanding Media, Dresden/Basel.

Morris, Merrill und Christine Ogan (1996): The Internet as Mass Medium, in: Journal of Communication, Jg. 46, S. 39-50.

Luhmann, Niklas (1984): Soziale Systeme. Grundriß einer allgemeinen Theorie, Frankfurt a.M.

Luhmann, Niklas (1995): Soziologische Aufklärung 6. Die Soziologie und der Mensch, Opladen.

Luhmann, Niklas (1996): Die Realität der Massenmedien, Opladen.

Paetau, Michael (1997): Sozialität in virtuellen Räumen? in: Becker, Barbara und Michael Paetau (Hrsg.): Virtualisienung des Sozialen. Die Informationsgesellschaft zwischen Fragmentierung und Globalisienung, Frankfurt a.M., S. 103-134.

Paetau, Michael (1999): Computernetzwerke und die Konstitution des Sozialen, in: Honegger, Claudia u.a. (Hrsg.): Grenzenlose Gesellschaft? Verhandlungen des 29. Kongresses der Deutschen Gesellschaft für Soziologie, des 16. Kongresses der Österreichischen Gesellschaft für Soziologie, des 11. Kongresses der Schweizerischen Gesellschaft für Soziologie in Freiburg i. Br. 1998, Opladen, S. 270-284.

Rammert, Werner (1998): Giddens und die Gesellschaft der Heinzelmännchen. Zur Soziologie technischer Agenten und Systeme verteilter Künstlicher Intelligenz, in: Malsch, Thomas (Hrsg.): Sozionik. Soziologische Ansichten über künstliche Sozialität, Berlin, S. 91-128.

Rheingold, Howard (1995): Die Zukunft der Demokratie und die vier Prinzipien der Computerkommunikation, in: Bollmann, Stefan (Hrsg.): Kursbuch Neue Medien. Trends in Wirtschaft und Politik, Wissenschaft und Kultur, Mannheim, S. 189-197.

Roesler, Alexander (1997): Bequeme Einmischung. Internet und Öfentlichkeit, in: Münker, Stefan und Alexander Roesler (Hrsg.): Mythos Internet, Frankfurt a.M., S. 171-192.

Runkehl, Jens u.a. (1998): Sprache und Kommunikation im Internet. Überblick und Analysen, Opladen.

Sassen, Saskia (1997): Cyber-Segmentierungen. Elektronischer Raum und Macht, in: Münker, Stefan und Alexander Roesler (Hrsg.): Mythos Internet, Frankfurt a.M., S. 215-235.

Schroer, Markus (2003): Raumgrenzen in Bewegung. Zur Interpretation realer und virtueller Räume, in: Sociologia Internationalis, Heft 1, S. 55-76.

Schwibbe, Gudrun und Ira Spieker (1999): Virtuelle Friedhöfe, in: Zeitschrift für Volkskunde, Jg. 95 , S. $220-245$.

Stichweh, Rudolf (2000): Die Weltgesellschaft. Soziologische Analysen, Frankfurt a.M.

Walther, Joseph B. (1996): Computer-Mediated Communication. Impersonal, Interpersonal and Hyperpersonal Interaction, in: Communication Research, Jg. 23, S. 3-43.

Wehner, Josef (1997a): Interaktive Medien - Ende der Massenkommunikation?, in: Zeitschrift für Soziologie, Jg. 26, S. 96-114.

Wehner, Josef (1997b): Medien als Kommunikationspartner. Zur Entstehung elektronischer Schriftlichkeit im Internet, in: Gräf, Lorenz und Markus Krajewski (Hrsg.): Soziologie des Internet. Handeln im elektronischen Web-Werk, Frankfurt a.M.NNew York, S. 125-150.

Winter, Carsten (1998): Internet/Online-Medien, in: Faulstich, Werner (Hrsg.): Grundwissen Medien, München, S. 274-295. 



\title{
YONERN ALS IRONISCHE RAUM(DE)KONSTRUKTION ETHNOGRAPHISCHE ERKUNDUNGEN IN VIRTUELLEN RÄUMEN
}

\author{
Sabine Thabe und Arne Schlechter
}

\section{VORBEMERKUNG}

„Wer das weisse Rauschen sieht, der wird sofort wahnsinnig. Ausser wenn er schon wahnsinnig ist. Dann wird er normal. " (http://www.dasweisserauschen.de)

Der vorliegende Beitrag beschäftigt sich mit den Erlebnissen und Ergebnissen einer dreimonatigen teilnehmenden Beobachtung von virtuellen Räumen innerhalb eines Kommunikationsforums im Internet. Angeknüpft wird in diesem Kontext an techniksoziologische Vorstudien und wissenschaftstheoretische Vorträge des Autorenteams über die zunehmende Bedeutung von virtuellen Räumen. ${ }^{1}$ Der Aufsatz reflektiert:

1. neue Kommunikationsformen im Internet,

2. Strukturen und Logiken virtueller Gemeinschaften,

3. die Relation von Raum - Netz - Subjekt.

\section{METHODOLOGISCHE RANDNOTIZ}

\begin{abstract}
„In meinen Träumen kann ich die Wahrheit sehen / in meinen Träumen kann ich nicht vor mir entfliehen / in meinen Träumen halt ich dem Spiegelbild nicht stand / in meinen Träumen da werd ich viel zu schnell erkannt."

(Tanzwut)
\end{abstract}

Die Untersuchung über die Forenwelt der Yoner umfasst die Zeitspanne von Mitte Mai bis Mitte Juli 2001. Als Einstieg in das Untersuchungsfeld wählten die Autoren (verdeckte) teilnehmende Beobachtungsformen, die sich, abhängig von den sehr unterschiedlichen Kommunikationspräferenzen des Autorenteams, kontrovers gestalteten: ${ }^{2}$ Während die Autorin einen hermeneutisch-empathischen

1 So etwa in den Seminaren „Mediale Räume - Reale Räume“ im Wintersemester 2000/2001 (Dozentinnen: Dipl.-Soz. Bettina Langenau und Dr. Sabine Thabe, Uni Dortmund) und „Wissenschaftstheorie in der Raumplanung" im Sommersemester 2001 (Dozenten: Prof. Dr. Michael Wegener, Dr. Hermann Bömer, PD Dr. Sabine Thabe, Uni Dortmund).

2 Die Beobachtungen umfassen 1. direkte (eingeschlossene) Beobachtung, 2. den Blick des ausgeschlossenen Dritten (Beobachter des Beobachters) und 3. gemeinsame Reflexionen über 
Schreibstil kultivierte, hielt sich der Autor von Beginn an auf ironischer Distanz zu den anderen Teilnehmern des Feldes und provozierte diese durch häufige Verweise auf (persönliche) Unzulänglichkeiten oder widersprüchliche Verhaltensweisen. Um vor den anderen Forenusern dennoch einen Eindruck von Gemeinsamkeit zu erhalten, schrieb das Team zwischenzeitlich mit Hilfe eines Sharenicks ${ }^{3}$.

Zusätzlich zur gemeinsamen teilnehmenden Beobachtung, die sich täglich mindestens vier bis sechs Stunden im Netz abspielte, kommunizierte der Autor via e-mail mit einer Hauptprotagonistin verschiedener Foren (soulkitchen) und die Autorin traf sich mit weiteren Usern (z.B. springender punkt und Zitzenspitz) zum Chatten per ICQ ${ }^{4}$.

Zusammenfassend kann von einem (zeit)intensiven Engagement im YonerForum gesprochen werden: Die Netzteilnahme begann morgens mit der Lektüre der Beiträge, die nachts in den Foren gepostet (= geschrieben) wurden. Je nach Bedeutung und Thema wurde zurückgeschrieben, so dass die/der Autor/in sich mittags/nachmittags mit den eingegangenen Replys befassen konnte. Am frühen Abend wurden die Foren erneut aufgesucht, zum einen, um den vollständigen Überblick über das Forengeschehen aufrechtzuerhalten, zum anderen, um Beiträge zu verfassen, auf die im Laufe des Abends seitens der anderen User reagiert werden konnte.

Der intensivste Kontakt mit den Teilnehmern der Foren ergab sich in der Zeit zwischen 22 Uhr abends und 1 Uhr morgens; zu diesem Zeitpunkt herrschte eine rege Teilnahme und bestand Interesse, über die verschiedensten Themen zu diskutieren. Ein harter Kern von Usern blieb oft noch bis drei, vier Uhr morgens, was für die Autorin/den Autor zeitweise eine sehr erschöpfende Teilnahme bedeutete, angesichts des Umstandes, am jeweils nächsten Morgen wieder pünktlich bei der Arbeit erscheinen zu müssen.

Die komplexe Präsenz im Netz veränderte zudem Wahrnehmungsstrukturen, die üblicherweise im real life nur selten hinterfragt werden und es erfolgten lange Reflexionen über die Unterschiede von real und virtual life (vgl. dazu Herz 1996). Nach dem ersten Monat intensiv-teilnehmender Beobachtung, die von der Autorin allein durchgeführt wurde, zeigten sich Formen des berüchtigten Forenkollers (d.h. Ausstiegsüberlegungen, Paranoia, Ärger mit Usern). Dies änderte sich wieder zum Positiven, als ab dem zweiten Untersuchungsmonat der Autor ins Feld eintrat. In puncto Interaktionen im Rahmen von identisch und/oder different bezog er eine klare Gegenposition. Dadurch wurden neue Offenheiten im Untersuchungsrahmen erzielt, die es zudem möglich machten, wesentlich differenzierter auf die einzelnen Forenteilnehmer einzugehen.

Beobachtungen dessen, was man nicht sieht, d.h. die „blinden Flecken“ von Kommunikationssystemen.

3 Ein Sharenick wird von mindestens zwei Usem (eines Forums) gemeinsam gebraucht und dokumentiert eine gewisse Vertrautheit untereinander, da das Passwort zum Einloggen des Nicks geteilt wird.

4 ICQ (http://web.icq.com/) bietet zahlreiche Kommunikationsmöglichkeiten von SMS, EMailservice, Newsgroups bis hin zu privaten Chatmöglichkeiten. 
Ab September 2001, nach Abschluss der empirischen Erhebungen, fand die Teilnahme an Yoner-Forentreffen in Berlin, Dortmund und Wien statt; darüber hinaus ergab sich ein regelmäßiger Austausch an Musik- und Fotomaterial, ${ }^{5}$ welches als wichtiger Bestandteil der Kommunikationskultur des Forums interpretiert werden kann.

\title{
3. LEBEN IM NETZ
}

\begin{abstract}
„Wir sind wie das Meer, wie die schäumende Gischt / die den Sand überspült und die Spuren verwischt / wir sind wie der Sturm, wie die Schreie im Wind / wie das Herz eines Narren, der zu tanzen beginnt. " (Tanzwut)
\end{abstract}

Sherry Turkles` prominente Studie „Leben im Netz“ (1998) bildete die Motivation, virtuelle Raumkonzepte subjektorientiert und im Selbstversuch zu reflektieren. Frei nach der AOL-Werbung (Boris Becker: „Ich bin drin!“) sollte im Selbsttest herausgefunden werden, was es heißt, im (virtuellen) Raum, eben drin, zu sein. Die Bedeutung des Subjektes ist dabei für die Gestaltung kommunikativer Prozesse samt ihrer virtuellen Räume immens, weil es neben den Betreibern von Kommunikationsforen (den sogenannten Webmastern), die die technische Infrastruktur liefern, (aus)schließlich die Forenuser selbst sind, die das kommunikative Geschehen in ihren Räumen (Foren) bestimmen. Die im Folgenden häufig auch als Poster bezeichneten Netzteilnehmer bilden virtuelle Gemeinschaften, die sich ganz bewusst ihre eigenen virtuellen Räume gestalten (vgl. Bollmann/Heibach 1998).

Untersucht wird in diesem Zusammenhang nicht das Verhalten in Chaträumen, sondern die Teilnahme an interaktiven Foren, in denen zu bestimmten Themen häufig lange Threads $^{6}$ über mehrere Tage geschrieben werden (wobei die Wahl der Themen autonom bestimmt wird). Hier dient das Internet als Spielfeld und Laboratorium für Identitäts(de)konstruktionen, die sich im Rahmen von kollektiv gestalteten virtuellen Räumen experimentell entfalten, oder, mit den Worten Sherry Turkles: Das Internet bietet Optionen, wie wir ,unsere eigenen Dramen projizieren, indem wir Produzent, Regisseur und Star in einem sind.“ Virtueller Raum (virtual life), als Parallelwelt zur realen Welt (real life) gedacht, unterliegt demnach vergleichbaren Strukturen und Logiken, mittels derer „Wirklichkeit" (de)konstruiert werden kann (vgl. Schlechter 2001; Thabe 2002), unterscheidet sich jedoch hinsichtlich einiger wesentlicher Details:

1. Nickname ${ }^{7}$ : Der Nick eines Users gibt in der Regel Auskunft über dessen narratives Selbstkonzept, denn mit der Wahl (s)eines Namens, der für die Teil-

5 Vgl. die Abbildungen in diesem Aufsatz und die Hinweise auf relevante CD's.

6 Unter einem Thread versteht man einen Diskussionsstrang, der mit dem sog. Headposting eröffnet und von verschiedenen User-Beiträgen fortgesetzt wird (z.B. Thema Raum und Traum).

7 Die Gruppen der Nicks lassen sich folgendermaßen unterteilen: 1. Mononicks (d.h. ausschließliche Verwendung eines Posternamens), 2. Multinicks (gemeint sind Nickfamilien bzw. die Verwendung unterschiedlicher Rollennamen), 3. Sharenicks (i.d.S. dass sich 
nahme am Forengeschehen obligatorisch ist, betreibt der User Selbst-Identifikation bzw. gibt Hinweise darauf, nach welchem Konzept und/oder nach welcher Rolle er/sie im virtuellen Raum seiner/ihrer community identifiziert werden möchte.

Von besonderer Bedeutung sind in diesem Zusammenhang die Charakternamen: Sie verweisen auf besonders geliebte Seiten des realen Charakters eines Users. Die Postings unter diesen Namen werden in der Regel nicht rollenfixiert geschrieben, sie benutzen allerdings charakteristische Äußerungen. Generell gehen sie auf jede für sie interessante Art von Dialogen ein und treten nicht nur bezogen auf ein spezifisches Thema auf.

Charakternamen sind häufig auch die Hauptnamen von Personen, also die Nicks, unter denen am häufigsten gepostet wird. Beispiele für Namen von Usern, mit denen die Autoren während ihrer empirischen Studien Kontakt hatten, wären z.B.: soulkitchen, dr_yoghurt, Zitzenspitz, springender_punkt, hohes_gericht und mindbender. Wie bereits erwähnt, benutzen (gerade diese) User ${ }^{8}$ aber häufig eine Reihe von Nebennamen, die je nach Gefühlszustand, Thema oder zur Verwirrung anderer Teilnehmer gewählt werden (z.B. zombieyoghurt, soulcrypta, kontrapunkt, spreewaldgurke). Auch werden die Nebennamen zur Ironisierung des Hauptnicks gewählt, etwa wenn sich dr_yoghurt als ,akademischeKlorolle“ bezeichnet oder soulkitchen mit dem Nick ,seelenabgrund“ schreibt. Für gelegentliche Rollenspiele werden Nicks generiert, die ausschließlich zu diesem Anlass gebraucht werden, z.B.: glottaler_gurrlaut, schwarzer_sheriff oder jule_kotzt.

Neben den (meistens) kreativen Rollenspielen tauchen auch User in der Forenwelt auf, die dort ihren Aggressionen freien Lauf lassen wollen oder ganz bewusst eine böse Rolle spielen (z.B. als Forenhenker, Bestatter oder interaktiver Vernichter). Bereits mit der Wahl des Nicks lassen sich diese User unschwer identifizieren, wenn sie sich etwa als Scheißer, Pullermann, das_böse_Etwas oder Forenterror bezeichnen. Insgesamt lässt sich festhalten, dass die Wahl des Nicks eine hohe Bedeutung für die Rolle, die man zu spielen gedenkt, darstellt. Dazu zählt insbesondere auch die Entscheidung, ob anhand des Namens z.B. Geschlecht, Alter, Ethnie etc. erkennbar werden sollen. ${ }^{9}$

2. Sprache: Die Sprachen von Kommunikationsforen unterscheiden sich sowohl von Alltags- als auch von Wissenschaftssprachen. Sie unterliegen selbstverständlich ebenfalls bildungs-, geschlechts- und schichtspezifischen Kategorien, übernehmen darüber hinaus aber in weiten Bereichen Stilelemente jugend- und subkultureller Sprachmilieus und Musikkulturen. Wichtig scheint hier insbesonde-

die Verwendung unterschiedlicher Rollennamen), 3. Sharenicks (i.d.S. dass sich mindestens zwei User einen Namen teilen) und 4. Fakenicks (d.h. Raubnamen, z.B. durch die Fälschung des Nicks eines anderen Users).

8 Es handelt sich dabei um Personen, die besonders häufig in den Foren auftreten und intensiv untereinander kommunizieren (die sog. Top-Poster im Forenranking).

9 Die Frage der Erkennbarkeit scheint letztlich schwierig zu beantworten, denn viele User switchen beim Posten ganz bewusst zwischen virtuellem Alter, Geschlecht, ethnischer Zugehörigkeit und ihren strukturellen Gebundenheiten im real life. 
re den gebildeten Teilnehmern, dass sie sich durch ein hochgradig individualisiertes Sprachgebaren von anderen Usern zu differenzieren verstehen.

Neben Details, die diverse Idiosynkrasien und Sprachverliebtheiten betreffen, wird häufig ausschließlich in Kleinschrift getextet und zudem die sogenannte Sternchenschrift zwecks Sprachverknappung verwendet. Beides dient einer weiteren Beschleunigung der Kommunikation (vergleichbar auch der SMS-Kommunikation) und lässt diese comicähnlich geraten, wie etwa folgende Beispiele dokumentieren: *michinsforumbegeb*, *daskaltegrauenbekomm*, *kopfschüttelndabwend*. Forensprachen changieren, dies nur als knappe Zusammenfassung, zwischen einfachen Zustimmungen (Zitzenspitz: „yep“) bis hin zu komplexen Formen von Ironie (x-nada: „Entschuldigen Sie, aber Sie haben eine flache Denke!")

3. Einsatz spezieller Symbole: Neben den Sprachverknappungen, wie sie sich durch Comicsprache und Sternchenschrift artikulieren, existiert ein reger Gebrauch an emos (emotional icons): Kürzel wie $(;)$ oder $(:)$ dokumentieren in einfachster Form den gefühlsmäßigen Zustand des jeweiligen Posters, der sich in der Schrift bisweilen differenzierter darstellen lässt und $\mathrm{zu}$ folgenden states führen kann: *betroffenblick*, *verliebtanzwinker* oder *vorwutindieluftgeh*. Neben diesen Kürzeln werden spezielle icons verwendet, die von den Forenadministratoren für die User zur Verfügung gestellt werden, und mittels derer Ansichten bildlich vermittelt werden sollen. Folgender Dialog, der von zwei Usern unter dem Sharenick „Seelentinte“ verfasst wurde, gibt Einblick in die Bildersprache des Yoner-Forums:

(blättert in einem alten lehrbuch für kriminologie): aha, da haben wir es! jetzt bin ich der seele auf der spur! angewachsene ohrläppchen deuten auf den hinterhältigen charakter eines ganoven!

$\$$ : und wenn schon? *unschuldigblick* meine blutspur ist null negativ!

6: *flugspurverwisch* *ablenk* und wie ist das mit der zähmung des widerspenstigen öhrchens?

tinten * (nasal): willst du körperöffnungen das abc beibringen? *buchstabenschnupfen* *andenspiongerichtet* schaun sie doch mal der $\mathbb{E}$ hinter die ohren!

4: schnuppern lass ich da aber nur

2. gewisse umgekehrte korrelationen zu anderen (w)orten sind mir yedenfalls schon aufgefallen ... *detailssittsamverschweig*

$\$$ : hilfe, jetzt werden hier nicht nur die uhren sondern auch noch die ohren verdreht. *q tippsuch* hihi, als bücherwurm kenn ich eh nur eselsohren.

\&: hilfe, meine ohren wurden von einer nase verdreht ! 
das nehme ich mit interesse zur kenntnis $\mathbb{B}$ ! hier liegt nämlich bereits eine anzeige wegen verführung rechtschlafender ohren vor.

*unschuldsblickaufsetz* lassen sie meine nase aus dem spiel, meine zähne sind rasiert!

kurzauftritt hai * : gestatten, mackie messer *wegschwimm*

: da sehen sie's herr $\mathbb{R}$ ! wer geht denn hier auf der spur der ganoven? *aufdie ${ }^{\text {Hodeut }}{ }^{*}$

: *moischenkleinmach* hi-hi-hilfee ! wo ist das necste wolkenloch, in das ich mich verfliegen kann?

*augenverdreh* wer weiss da noch rat?

: uhu ropax!

Eine kurze Geschichte der anstehenden Analyse virtueller Räume wird im Folgenden verdeutlichen, wie zentral postmoderne Begriffe wie Kreativität, Intuition und Spontaneität für eine zeitgemäße Wissenschaftskultur sind, die sich insbesondere von sprachphilosophischen, tiefenpsychologischen und kulturtheoretischen Prämissen her definiert und in ihrer Empirie subjektorientiert begreift (vgl. Thabe 2002).

\title{
4. ANKUNFT IM YONER-LAND
}

\begin{abstract}
„Am Eingang steh' $n$ wir - angekommen / Dieses soll Dein Anfang sein / Auserwählt und auserkoren / Hineingeborn lass ich Dich jetzt allein / Nun versuchst Du deinen Weg zu erkennen / Zwischen zahllosen Röhren, Schächten und Gängen / Irrst Du umher, dann siehst Du das Licht / So sehr Du auch rennst, Du erreichst es nicht / Im Labyrinth der Sinne - In den Wirren Deiner Seele bist du ganz allein / Im Labyrinth der Sinne - Nur wer beflügelt, wird sich selbst des Rätsels Lösung sein. " (Tanzwut)
\end{abstract}

Mitte Mai 2001 betrat die Autorin zum ersten Mal den virtuellen Raum der Yoner, indem sie dort ein Headposting ${ }^{10}$ platzierte. Noch am selben Abend schrieben drei anwesende Yoner (soulcrypta, crazy und dr_yoghurt) zurück und signalisierten Interesse an der Frage, wer sie sind. Die Frage selbst wurde recht unmittelbar als Bestätigung ihrer (bewussten) Anwesenheit in einem virtuellen Raum verstanden,

10 Ein Headposting ist ein Beitrag, der weitere Postings initiiert. Unter dem Titel „Invokation der Anwesung" schrieb die Autorin Folgendes an die Yoner: „Hi, ihr Yoner, bin neu hier *staun* Dumme Frage: Wer seid Ihr?" 
den alle drei User jedoch an eine alltägliche Lebenswelt zurückbanden, nicht ohne auf deren Virtualität hinzuweisen.

So antwortete die erste Posterin: ,yo, machs dir gemütlich ... magst aufs sofa, oder hast du lieber orangenboimchen, yuccas oder philodendron? wie hat es dich eigentlich auf diesen planeten verschlagen? gut, dass es die schnellen ufos gibt, da kannst du yo immer wieder mal vorbeidüsen“ (soulcrypta). An die Vorstellung, sich auf einem eigenen Planeten zu befinden, knüpfte ein zweiter Beitrag an, der Folgendes überlegt: „Sind wir nicht alle von einem ganz speziellen Planeten? Tintenaffe vom Planeten der Affen und Crazy vom Planet der Verrückten und Soulcrypta ... hm ... vom Planeten der Seelen?“ (crazy) Nach einigem Hin und Her darüber, wo wir sind, wenn wir sind, beschloss ein drittes Forenmitglied die abendliche Diskussion mit dem Hinweis: „wir sind virtuell unverwüstlich ... wenn wir mal keine lust haben, schlafen wir drei tage aus ... dann kehren wir zurück" (dr_yoghurt).

Diskussionen dieser Art setzten sich an den darauf folgenden Abenden fort. Auf Fragen der Autorin (z.B. „Ist noch wer wach?") bekam sie regelmäßig Antworten, die eine Lokalisierung der User implizierten. Ein Beispiel mag dies verdeutlichen:

Autorin: „Echt, alle schlafen?“

soulkitchen: „nein, sie kriechen nur langsam aus ihren löchern.“

Autorin: „Aus welchen Löchern denn?“

soulkitchen: ,also, ich bin aus dem loch dahinten gekrochen. *indennebeldoit*.“

Durch gezielt beiläufiges Fragen, wo wir denn nun sind, wenn wir uns in virtuellen Räumen bewegen, bekam die Autorin in den ersten beiden Wochen ihrer aktiven Forenteilnahme weitere Kontakte zu Usern, die sich offensichtlich ebenfalls an diesem (Raum-)Thema beteiligen wollten.

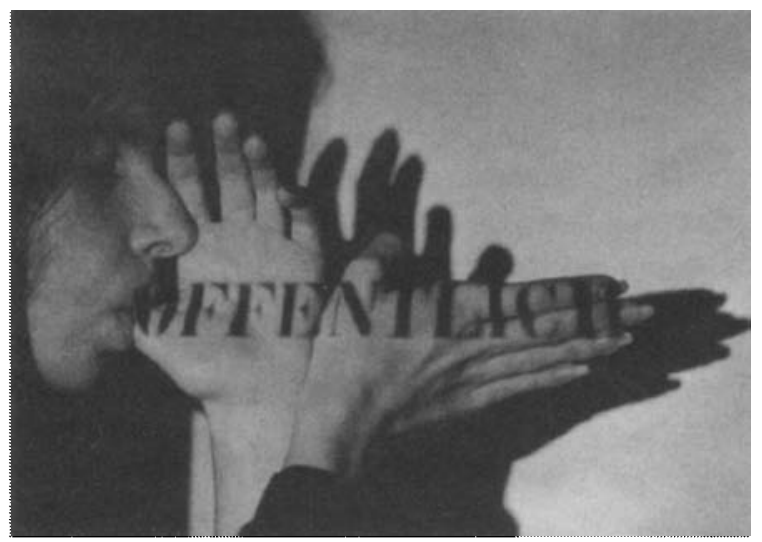

Abbildung 1: öffentlich oder privat? [soulkitchen] (Quelle: Archiv Soulkitchen). 
Die Kontaktaufnahme gestaltete sich dabei (wie in der ,realen“ Welt aucı) abhängig vom Selbstbild der Kommunizierenden, z.B. komisch: „Darf ich mich vorstellen: Mein Name ist Zitzenspitz ... vom Clan der hankanischen fiesen Gurkenfresser ... und ich bin der einzige Angehörige dieses Clans" (Zitzenspitz). En anderer Poster outet in diesem Zusammenhang einige seiner früheren Aktivitäten im Forum: „Ich war die Dönerpolizei!“ (hohes_gericht) Ernsthaftere Beiträge thematisieren den experimentellen Charakter virtueller Räume: „Was ist diesss cyber -tun, wenn nicht ein gestörtes probehandeln für uns verhaltensgestörte"“ (kinglear) Oder sie greifen das Thema von seiner lyrischen Seite her auf: „DER RING DER GRÜNEN LICHTER / DER KING DER ROTEN DICHTER / DER KONG DER TOTEN RICHTER“ (mindbender).

Ernsthaftigkeit und Eulenspiegelei, so zeigte sich bereits in den ersten beiden Wochen der Forenpräsenz, das sind zwei Welten, mit denen die Yoner bewusst spielen. So äußert dr_yoghurt ernsthaft und selbstreflexiv: ,die virtuelle welt st für mich ein pendeln zwischen dem in mir, das authentizität, sagen wir mal wahheit, will, braucht, sucht. und dem andern, das sich schützt; gewillt ist, süchtg, eben tot. tot und lebendig, beides zugleich. so faken wir uns selber. PAIRfekt" Nichtsdestotrotz (oder vielleicht auch gerade deshalb) versetzt er diese relativisische Sicht mit einer ironischen Komik, die man vielen Wissenschaftlern für ihe erkenntnistheoretischen Positionen nur wünschen kann, denn ,man kann letztlich nie wissen, ob am anderen compi ein huhn oder ein zebrafink postet, der nır vorgibt, ein wiener oder frankfurter zu sein" (dr_yoghurt).

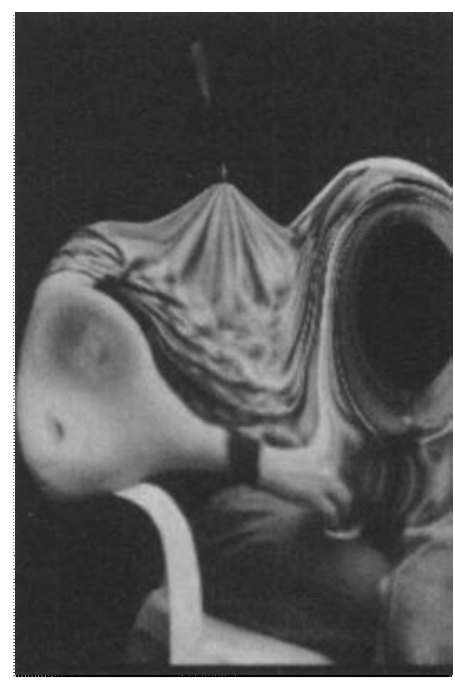

Abbildung 2: liquidierung eines wiener posters [dr yoghurt] (Quelle: Archv Soulkitchen).

Ähnliche Sprachspiele zur Verzauberung und Infragestellung von common sense dekliniert soulkitchen, die über sich selber schreibt: „küchen schlafen bei 
offenem fenster und klappern mit ihren tassen." Was sich hier spielerisch verkehrt, ist eine scheinbar geordnete Welt, die eine weitere Userin folgendermaßen zum Tanzen bringt: „den zixsten zen ver-zücken / den zexten zinn ver-zinken“ (Tintenaffe).

\section{SELBST-VERSTÄNDNIS UND SPRACHE}

"und wach icht, wenn der bauch dech walfich chelbcht anfängt chu träumen? fühlt er chisch dann wie eine note im gefieder einech chingvogelch?"

(walfich)

Im Rahmen täglicher, mehrstündiger Kontakte mit den Yonern wurden diese immer vertrauter mit der Autorin, denn zusehends bezogen sie diese in ihre Welt ein und nahmen sie in ihre Gemeinschaft auf. Ein Teil diverser Aufnahmerituale bestand darin, mit den Yoner-Mythen, die sie in einem eigenen Forenlexikon angelegt haben, vertraut gemacht zu werden sowie in der Einladung, in ihrem autonom betriebenen Forum zu schreiben. So wurde es möglich, sich einerseits vertraut und andererseits distanziert ethnographisch mit der Forenwelt dieser virtuellen Gemeinschaft zu befassen, die mittels ihrer eigenen Sprache neue Formen von Identität (im Raum) experimentell erprobt.

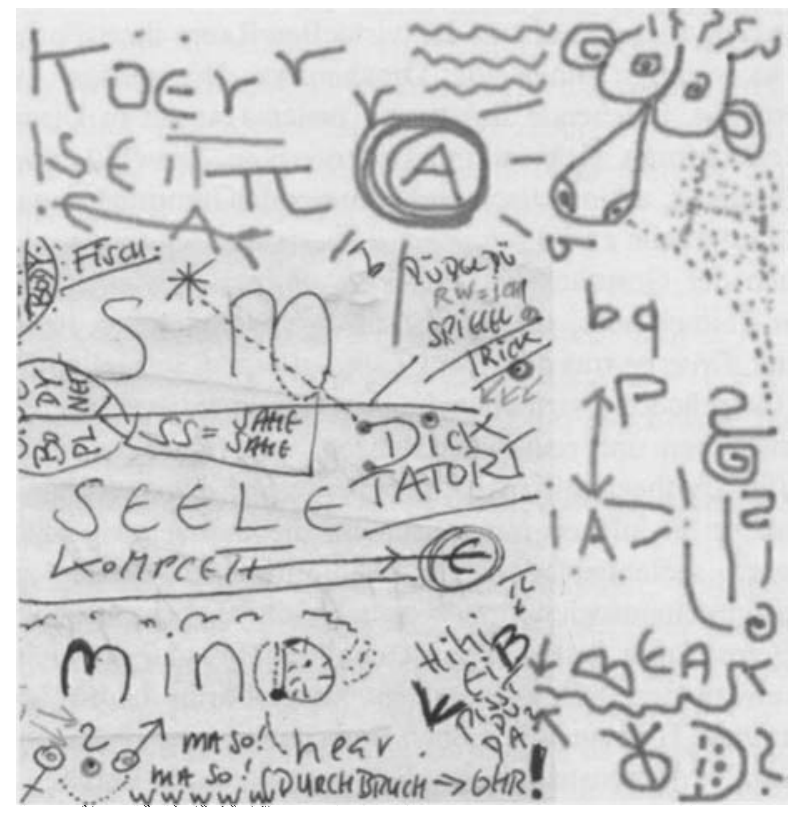

Abbildung 3: hinKunft [mindbender] (Quelle: Archiv Soulkitchen). 
Ein Blick in das Forenlexikon der Yoner zeigt, wie im Internet mit Rollen gespielt werden kann und Sprachgrenzen sich auflösen, wenn yonern zugelassen wird: So spielen Yoner mit diversen Alter Ego-Modellen (in ihrer Sprache: der Agent, das Dschenneln) und thematisieren die alte lacansche Debatte um die Illusionen eines Spiegel-Ich neu, indem sie von vornherein eine abduktive Sprachlogik betreiben, die alte, bekannte Konzepte überschreibt.

Als Beispiel sei das „fnatten" genannt, eine „for allem fon flugfesen beforzugte spfrache, die wunderbar die yonafistische sfprachlandschaft bereichert fhat" (flederfeger). Auch das ,walfichen“, eine ,agentich verchlüchelte chprache der meehrechbewohner" (walfich), ist Teil neuer Sprachspiele, die Raum(de)konstruktionen kreativ und dynamisch betreiben (vgl. Thabe 2002). Mit welchen Themen dies geschieht, wird im Folgenden genauer beschrieben, wenn dargelegt wird, wie die weiteren Annäherungen zwischen Autorenteam und Yonern stattfanden.

\section{ANNÄHERUNGEN}

„yo, wenn man beim ankommen kommt, dann war's auf yeden fall die richtige richtung, in die man geflogen ist. " (soulkitchen)

In den nächsten Wochen lieferten sich Autorin und Autor Abend für Abend Wortspiele mit den Yonern und nutzten den virtuellen Raum ihres Forums, um endlich einmal über so wichtige Dinge wie: Drachentinte, Wassertiger, Nebelschlangen, nekrophile Frösche, rauchende Eskimos, Tiroler Transen in Dirndln, postapokalyptische Affendoktoren, Kakteen mit Ledermasken, Kirschkernkanonen, infernalische Tintenjoghurts, aktionistisch menstruierende Gummibäume und vieles mehr stundenlange Gespräche zu führen.

Im Rahmen der Gesprächsverläufe kam es immer wieder zu Annäherungen zwischen den Teilnehmern, die sich ,räumlich“ lokalisieren ließen, etwa, wenn man sich in der Taverne traf oder zum Talk auf einem virtuellen Plüschsofa Platz nahm. Auch Gespräche in virtuellen Küchen dokumentieren fließende Übergänge zwischen künstlichen und realen Existenzen, wie folgendes Beispiel vermitteln soll. Tintenaffe: „erdbeer mir mehr, sprach lizzard; sonst entbär ich dich seehr, sprach tintenmeer: in küchen mit muscheln, die erdbeeren tuscheln. seelenstern mag erdbär gern. seelengebärdenspiel.“ Woraufhin soulkitchen zustimmend postet: „,"klingelninmeinenseelenohren*yo, auchküchenhabenmuscheln*sehsterne*."

Bei der Betrachtung und Analyse einzelner Threads, die sich zwischen den Teilnehmern entspinnen, fällt auf, dass im Yoner-Forum häufig über große, menschlich-berührende Themen wie Leben und Tod, Liebe, Nähe und Distanz mit Hilfe neuer Sprach(de)konstruktionen diskutiert wird. Im Rahmen virtueller Räume scheint es vor diesem Hintergnund sehr effektiv, sich kreativ mit Selbst(De)Konstruktionen und Räumen zu befassen. Die Yoner lösen somit erkenntnistheoretische Probleme, indem sie ihre komplexen Idiosynkrasien zum Ausgangs- 
punkt sprachphilosophischer Überlegungen nehmen und durch eine gleichsam spielerische Weise transformieren.

\title{
7. KLEINER EXKURS ÜBER DAS YONANIEREN
}

\author{
„Sometime ago I had a dream \\ It was happy it was lasting \\ It was free \\ And now in life $O$ can't you see \\ How we can make that dream \\ Into reality."
}

(Chick Corea)

Sich selbst persiflierend, definieren Yoner in ihrem Forenlexikon die große Bedeutung des yonanierens: „,mit sich selber beschäftigen ... genüsslich zeit mit sich verbringen ... in sich hören und den rufen von körper und seele antworten" (dr_yoghurt). Eine Hauptprotagonistin schreibt zu diesem Thema: „yoners ruuulllllleezzz ... hihi, früh morgens um $13 \mathrm{~h} 12$. in den foren herrscht totenstille. man hört nur eine gurke, die beim yonanieren ein bissl laut is ... *animalischegurkenlautevonmirgeb*" (yonanierende_gurke).

Yonanie, eine kreative Form der Selbst-Erzeugung und Selbst-Beobachtung (im Rahmen Neuer Medien), reflektiert ein weiteres Mitglied des Forums, wenn es in einen Dialog mit sich selber tritt, indem es seinen Hauptnick (dr_yoghurt) in zwei Unternicks spaltet. Mit dieser Liquidierung einer vormals kompakten IchStruktur zugunsten eines Alter Ego-Modells, das mit Dis lokation experimentiert, gelingt die Dekonstruktion vermeintlich fixer Identität:

dr_yoghurt (1): *imbecherchenrumhypf*

*diefruchtstückchenschizoschwärmeummichkreisen* hihi ...

*einpaarkroitleinseinrühr* $\mathrm{g}^{*}$ yetzt isses gemütlich ;-)

dr_yoghurt (2): ganz schön teuer auf dauer ... 2 chello-anschlüsse, bloß um mich selber zu faken *ROFL*

dr_yoghurt (1): yo, aber so wahnsinnigen wie mir (*wiederinsperichverfall*) is yo eh ned übern weg zu traun ...

dr yoghurt (2): yetzt mach ich mit mir selber einen thread ... und wechsle dazwischen das chello-modem, um vorzutäuschen, ich sei zwei wiener ... ${ }^{*} \operatorname{lol}^{*} \mathrm{y}$ etzt weiß ich endlich, was ich und ich bin: ein gehim mit zwei chello-anschlüssen * rofl*

dr yoghurt (1): yo, aber dafür mit hoher hirnkapazität, da braucht ma schon zwei anschlüsse ... hihi ... und außerdem will ich yo nur tarnen und verschleiern, weil ich was zu yerbergen hab * $\mathrm{g}^{*}$

dr_yoghurt (2): was soll man auch von mir schlankem, aber herzeigbaren doppelchelloanschlussinhaber erwarten? ${ }^{*}$ rofl*

dr_yoghurt (1): yo, meine herzeigbarkeit verschleier ich echt gut ... ${ }^{*} \mathrm{~g} *$ 


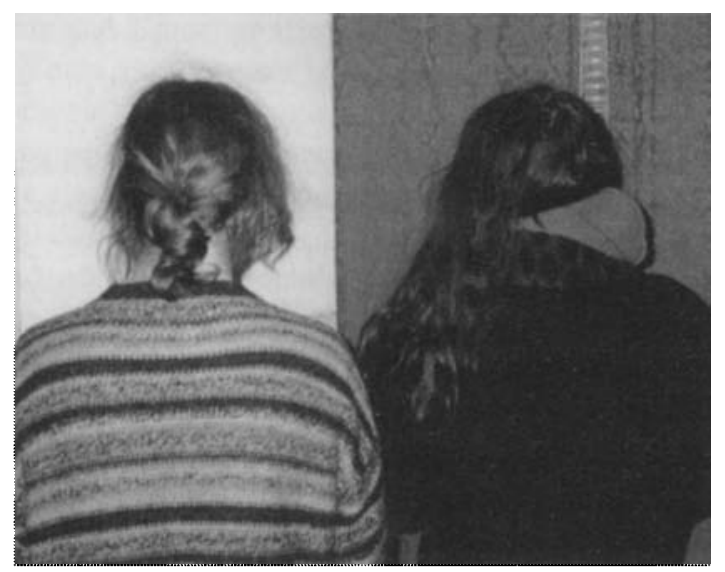

Abbildung 4: alter \& ego [doc_brown] (Quelle: Archiv Soulkitchen).

Sexualisierungen in Form von Sprachspielen in virtuellen Räumen werden von den Yonern in zwei weiteren Formen verwendet: Die erste bezieht sich auf den sogenannten Botanomismus, der als Liebe zu und mit Pflanzen ironisiert wird: „,mein philodendron hat mich verführt ... ganz aufreizend mit den blättchen gewackelt hat er und die luftwurzeln immer so in meine richtung gesteckt ... ich konnte ihm einfach nicht widerstehen" (soulkitchen). Absurditäten dieser Art werden bis zum Ende spielerisch bearbeitet: „von einer unersättlichen yucca sexuell bedrängt ... ich sehe schon die schlagzeilen: mensch von yucca vergewaltigt! 5 jahre haft für menschenschänderyucca" (soulkitchen).

Die zweite Form weiterer Persiflagen auf genormte Wirklichkeiten von Gesellschaft finden sich in den Ironisierungen von Uniformierungen. Eine Teilnehmerin von Europride 2001 in Wien ${ }^{11}$ statet dazu (über die dort anwesende Polizei): „die hätten auch mal statt ihrer uniformhose ein sonnenblümchen im schritt haben können zur feier des tages" und sie schlägt vor "früchte, kroiter und frösche" auf die Uniformen zu nähen. Ironie und Komik, die hier zum Ausdruck kommen, finden sich auch in den nächtlichen Gesprächen der Yoner über Raum. Als Beispiel soll hier eine kurze Geschichte über ein Toilettenhäuschen auf einem öffentlichen Platz dienen, die von zwei Forenusern aus Wien eines Abends erzählt wurde, nachdem die Geschichte am Tag vorher durch die lokale Presse ging.

11 Europride 2001, ein schwul-lesbisches Event, lässt sich am besten mit dem Christopher Street Day vergleichen, der seine Tradition aus der Geschichte der amerikanischen Homosexuellenbewegung bezieht. 


\section{VERYONERT IN WIEN ODER: IRONIE IN DER GROßSTADT}

„wenn der rock' $n$ roll nicht zum körper kommt $\otimes$ dann kommt, hihi, vielleicht der körper $\odot$ nach WIEN. " (mindbender)

Im Zuge einer weiteren abendlichen Diskussion zwischen soulkitchen, dr_yoghurt und Tintenaffe kommen die drei User auf das Thema Wien zu sprechen. dr_yoghurt, der unweit von der Berggasse ${ }^{12}$ wohnt, schlägt vor, ein Kaffeehaus für „Freaks“ zu eröffnen und dieses als „Neurosenurquell“ oder „Borderline-Bar“ zu betreiben. Tintenaffe folgt dieser Idee zuerst und erweitert sie dann um den Gedanken eine virtuelle Praxisgemeinschaft zu gründen, mit folgenden Fachrichtungen: Dr. Soulkitchen (Pilzbeschwörung), Dr. Yoghurt (Fruchtzwergtherapie) und Dr. Tintenaffe (Nebenhöhlenvereitelung). Nach diesen ersten Plänkeleien setzen die beiden Wiener Poster ihr Gespräch fort.

dr_yoghurt: „warum san wir wiener wirklich so besonders komisch und debat?" soulkitchen greift die Frage auf, wenn sie reflektiert: „ich weiß ja aa ned, ob die loit anderswo weniger debbad sind oder nur so tun ... im notfall däd ich's auf die habsburger schieben, auf die lippizaner und mozart's kleine nachtmusik, da kann man ya nur debbad werden ... nicht umsonst ist die noirose in wien entdeckt worden, oder?" Nachdem sich die beiden eine Weile damit auseinandergesetzt haben, ob die Neurose nun erfunden oder entdeckt wurde, kommen sie zum Thema Wien zurück.

Dazu erzählt dr_yoghurt folgende Geschichte: „letzte woche fanden sie in letzter minute einen mann, der drei tage in einem mobilklo eingesperrt war. mitten auf der straße und keiner hat's gemerkt! nachdem er ausgeraubt worden war, wollte er in das klo flüchten. die „räuber“ warfen daraufhin das klo kurzerhand um und flohen. das klo fiel jetzt aber so unglücklich, dass die tür nicht mehr aufging. gleich daneben ist eine tag- und nachtbaustelle, die angeblich ein schreien oder klopfen ausm klo unhörbar macht. nach drei tagen gelang`s ihm eine hand rauszustrecken, da hat dann jemand geschaltet, in dem umgefallenen klo ist jemand. knapp hat er's überlebt (war ja drei tage ohne essen und trinken)."

soulkitchen kontert in ihrer anschließenden Reaktion mit einem Hinweis auf wienerische Mentalität, die gern zur (öffentlichen) Ordnung ruft und wundert sich, warum dies nicht auch für Klohäuschen gelten soll: ,is schon interessant ... wenn in wien eine öffentliche uhr nachgeht oder stehnbleibt, dann melden das gleich unzählige loit angeblich ... ein umgestürztes mobilklo (egal, ob mit oder ohne mensch drinnen) sollte doch genauso viel aufmerksamkeit erregen?"

Die inhärente Kritik an einer achtlos-anonymen Großstadt setzt dr_yoghurt mit seinem folgenden Beitrag fort: „typisch wien halt ... gegen eine uhr, die vor oder nach geht, hat so ein schreiendes mobilklo einfach keine chance." Die Art und Weise, mit der hier ironisch-komisch (Gesellschafts-)Kritik geübt wird, kann noch weitergesponnen werden, denn uber das Thema Wien hinaus gibt soulkit-

12 In der Berggasse (Wien, IX. Bezirk) befand sich viele Jahre die psychoanalytische Praxis Sigmund Freuds. 
chen folgende Geschichte zum besten: ,... das erinnert mich ein bissl an die geschichte, wo ein typ in so einer weingegend so besoffen im unbeheizten vorraum umgekippt ist und drei tage da gelegen ist ... der sohn ist mehrmals täglich drübergestiegen und hat ned gemerkt, dass der vater schon tot ist ... der hat sich halt gedacht ... nayo, is halt wieder ein gscheider rausch, den der ausschläft, oder so ... wie selbstverständlich es für manche loit ist über bsoffene verwandte einfach drüberzusteigen ... oder an schreienden klos einfach vorbeizurennen.“

Yonerische Gesellschaftskritik praktiziert sich, wie die beiden Beispiele demonstrieren, indem sie vom Absurden her ihren Blick auf die vermeintlich ordentliche Wirklichkeit rigider Raumordnungen richtet: Toiletten werden dabei gegen Uhren gesetzt und Söhne steigen tagelang über ihre toten Väter ... die Räume, in denen so etwas passiert, sind jedenfalls alles andere als sicher!

\section{DAS YONER-FORUM: EIN LUMPEN- UND SCHELMENLAND}

„wir wollen gras für habermas! schlotter dyke: so, ein süppchen für mein püppchen. und nen keks bevor gebrezelt wird. *hihi* haber gay: (b)rätsel (s)peter? maserst du mich jetzt, dann schlotter ich dich later. " (Tintenaffe)

Was konnten drei Monate Forschungsaufenthalt bei den Yonern uns Reisenden nun vermitteln? Die Zeit im Yoner-Forum war sicherlich eine intensive und aus einigen virtuellen Kontakten ergaben sich schließlich reale Treffen und Freundschaften. Aus anfänglichen Frotzeleien über die Batiklogik postmoderner Hippies (wie es dem Autorenteam vorkam) wurde ein ernsthaftes Verstehenwollen der Forenbewohner, d.h. in ihrer Sprache und ihrer eigenen ironischen Komik: ,... aber weil eben die ... recht streitsüchtig warn, hatten wir schnell den ruf 'friedliebend' zu sein und halt ein bissl versponnen und knuddelig ... die forenhippies eben und so was veranlasst schon dazu, die loite mit ihren eigenen vorurteilen ein wenig zu kitzeln“ (Interview mit soulkitchen).

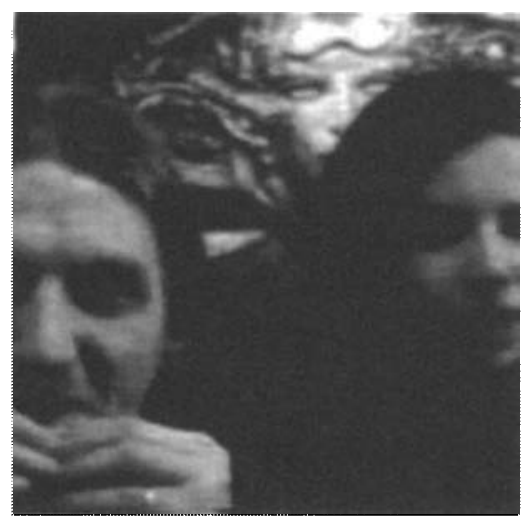

Abbildung 5: drei halbe [mb \& soul] (Quelle: Archiv Soulkitchen). 
Der Umgang mit Vorurteilen gegenüber den Bewohnern virtueller Räume veränderte sich auch seitens des Autorenteams; so gestand die Autorin im ICQChat mit soulkitchen: „Ich fühle mich schon total veryonert! Gehöre ich jetzt zu Euch?" Auch der Autor setzte sich intensiv mit "unserer" yonerischen Hauptinformantin soulkitchen auseinander, was sich in folgenden e-mail-Sequenzen dokumentiert.

soulkitchen schreibt dem Autor über kommunikative Annäherungen, die während dieser Zeit (aus ihrer Sicht) stattgefunden haben, Folgendes: „es ist so was wie ein näherkommen oder ein auseinanderdriften auf sprachlicher ebene, nicht rein inhaltlich ... obwohl beides oft gekoppelt ist, insofern als dass sich ein inhaltliches näherkommen oder voneinander entfernen auch auf sprachlicher ebene auswirkt." Gleichzeitig räumt sie ein: ,ich weiß, dass du das anders siehst, zumindest kann ich mich an ein paar deiner andeutungen (...) erinnern, wo dieses thema am rande gestreift wurde (...) und du dem etwas missbilligend gegenübergestanden bist.“ Abschließend gibt sie dem Autor folgende Spiegelung: „...trotzdem finde ich, kannst auch du dich von dieser eigendynamik der sprache nicht ganz ausnehmen, oder du hast in der letzten zeit wirklich eine gewisse wandlung in dir durchgemacht (...), aber ich finde schon, dass sich im laufe der letzen mails und auch im forum deine sprache etwas geändert hat (...) es geht mir mehr um den klang der zwischenzeilen, oder auch die grundfärbung, na, auf yeden fall war das ein starker eindruck von mir ... es hat sich schon was verändert, wenn ich yetzt stichelig sein wollte, würde ich meinen, du bist beyonert (...) is sicher etwas übertrieben und nicht ganz ernst, im grunde aber anerkennend und lieb gemeint.“

Auf dieses lobend-anerkennende Kompliment seitens seiner Gesprächspartnerin reagiert der angesprochene Autor (aufgrund seiner Furcht vor einem going native) in einem kruden Mix von distanzierter Ironie und Komik: „aber ich muss doch sehr bitten! natürlich hat sich meine art der kommunikation im forum geändert, ich bin ja schließlich nicht unflexibel. ABER ICH BIN AUF GAR KEINEN FALL BEYONERT!!!!! das ist ja furchtbar. da kann ich mich ja gleich einweisen lassen. auweia. jetzt muss ich auch noch wegen einer fachlichen arbeit meinen geisteszustand überprüfen lassen."

Die angeführten Autorenbeispiele sollen vermitteln, wie verschieden die $\mathrm{Zu}$ gänge zum Feld sein können und dass dennoch kreativ mit solchen Gegensätzen gearbeitet werden kann, wenn man diese als komplementär begreift. Unabhängig von diesen (subjektiv) verschiedenen Zugangsformen wurde deutlich, dass im Rahmen des Engagements in einem Forum eher die Zeit als der Raum eine wesentliche Bedeutung einnimmt. Während die virtuellen Räume lediglich die Kulissen für (Rollen-)Spiele der Yoner bieten, ist eine Verständigung über gemeinsame Zeiten, zu denen sich die User im Netz befinden und begegnen, von erheblicher Bedeutung, wenn gemeinsam und gleichzeitig kommuniziert werden soll. Der Umstand, sich zum Posten an einem Ort und vor einem Computer einzufinden, ist demnach, was seine Raumrelevanz betrifft, so trivial, wie das Argument, zum Waschen von Socken sei die Standortgebundenheit von Waschmaschinen die handlungstheoretische Prämisse. 
Die ironisch-komische Verwendung von Raum-Metaphern im Sprachgebrauch der Yoner reflektiert (nach den empirischen Erfahrungen des Autorenteams) eine zutiefst demokratische Kommunikationskultur, die sich in einem „Patchwork der Minderheiten" (Lyotard) dokumentiert. So wird es schließlich möglich, dass Wassertiger mit Nebelschlangen in Erdlöchem flirten, lammfromme Mohairpullover sich in Baumhäusern verstecken oder Affenbrötchen nach Seelenbeeren als Belag fragen. Der anarchistisch-liebevolle Denkstil und die liberal geprägte kommunikative Kompetenz der überwiegenden Zahl der Forenuser dokumentieren darüber hinaus, dass insbesondere im Rahmen einer distanziert-ironischen Relation zum bekannten Mainstream soziologischer Raum-Zeit-Konzeptionen weit über deren Begrenzungen hinausgedacht werden kann, wenn spielerisch-reflexive Inszenierungen zum Einsatz kommen.

"Ich ist ein anderer", das wusste schon Arthur Rimbaud, und so sind die virtuellen Räume der Yoner diesen einerseits inwendig vertraut, entspringen sie doch schließlich ihren privaten Idiosynkrasien, sowie gleichzeitig (ver)fremd(et) und damit geeignet als evokatives Potential für (Rollen-)Spiele, die räumliche Positionierungen spielerisch in Bewegung halten. Während im aktuellen (stadt)soziologischen common sense ein altgeographisch anmutendes Räumeln mittlerweile bis in die virtuellen Räume hineinreicht (vgl. Funken/Löw 2002, S. 69 ff.), plädieren die Yoner für experimentelles Handeln und sprachliches Spiel, z.B. wenn sie darlegen: „yonern ist nicht erklärbar - das ist ein gefühl, ein tun ohne anleitung, anarchisch, friedvoll ... frei liebend, grenzenlos ... paradox-genialunbegreiflich-universell-phantastisch-kreativ-triebauslebend; einfach eine art der kommunikation, die unbegreiflich und nicht erlernbar, aber spürbar und in jedem vorhanden ist ... yonern ist eigentlich eine form des sich-selber-ausdrückens an die umwelt ... und zwar so frei wie möglich von vorgegebenen zwängen" (crazy \& dr_yoghurt).

Yonern expliziert demnach Verbindungen zwischen Raum, Subjekt und Netz als Zwischenräume, als Freiflächen für Phantasien, mittels derer an der Erneuerung von Bewusstseins- und Erkenntnismodellen gearbeitet werden kann. Raum findet in diesem Sinne seine kreative Verwendung als „Übergangsobjekt“ (Winnicott) für Spieler, die als „Schwellenhüter und Vermittlungsfiguren“ (Adamowsky) im Grenzland zwischen dem realen Hier und dem anderen Dort (als einem Land hinter den Spiegeln) fluktuieren.

In diesem Raum-Zeit-Moratorium, so die Kulturwissenschaftlerin Natascha Adamowsky, ist „Spiel weder wahr noch falsch, weder angenehm noch schmerzhaft, sondern verweist auf die unausweichliche Kraft der Imagination. Es ist die wunderbare Gelegenheit,' sein Schicksal, seine Sehnsüchte, Wünsche, Leidenschaften in flüssige Luft zu schreiben, daran zu glauben und nicht daran zu glauben“ (Adamowsky 2000, S. 112). Welche Konsequenzen sich daraus für zukünftige „Versuche über den Raum“ (Kamper/Fecht) ergeben, darauf soll abschließend skizzenhaft eingegangen werden. 


\section{RAUM(DE)KONSTRUKTIONEN}

"It was a large room. Full of people. All kinds.

And they had all arrived at the same building

at more or less the same time.

And they were all free. And they were all

asking themselves the same question:

What is behind that curtain?

You were born. And so you're free. So happy birthday."

(Laurie Anderson)

Die Ethno- und Topographie des Yoner-Forums mag verdeutlichen, dass sich gegenwärtig Diskurspraktiken und Sprachpolitiken in virtuellen Räumen entwickeln, die parallel zu gesellschaftlichen Veränderungen von Raum(de)konstruktionen (vgl. Thabe 2002) ihre Entfaltung nehmen. Virtuelle Räume indizieren in diesem Kontext neue Schrift- und Lese-(Raum-)Kulturen, die sich insbesondere mit Fragen der Metakommunikation und Ästhetik befassen. Ihre (teilweise) avantgardistische Artikulation umfasst Heteroglossien (vgl. Bachtin/Grübel 2001) und Heterotopien (vgl. Foucault 1990).

Die erkenntnistheoretische Grundlage dieser neuen Kommunikationsformen manifestiert sich in einem ironisch-komischen Stil vieler User, die ganz bewusst mit virtuellen Selbst-Konstruktionen experimentieren: „Das Netz ist das Land der erfundenen Identitäten. Hier ist das Phantastische am Platz; es ist das Reich der Illusionen, des Fabulierens und Verzauberns. Kaum eine Metapher von Vielfalt und Wandel, die hier nicht anwendbar wäre. Der Computer als gateway in die digitalen Datennetze führt in eine Welt der Übergangsphänomene, die für diejenigen, die sich darauf einlassen, intensive Imaginationsstimulation bereithält" (Adamowsky 2000, S. 198, kursiv im Original).

Das Internet vermittelt (in diesem Sinne) geeignete Bühnen für Raum-NetzNarrationen, mit denen schreibende Subjekte ihre Pluralität artikulieren. Narrative Selbst-Konstruktionen, z.B. der Yoner-Community, verdeutlichen in komplexanschaulicher Form den Stellenwert einer theoretisch reflektierten Konzeption von Subjektivität, (auch) was die Entwicklung neuer Raumtheorien betrifft. Was in der diskursiven Praxis virtueller Räume seinen (experimentellen) Ausgang nimmt, sollte deshalb verstärkt theoretische Reflexionen und einen erweiterten Methodengebrauch in der Ausübung soziologischer Analysen anregen. Theoretische Reflexion würde in diesem Kontext bedeuten, formal sichere Glaubenssysteme in den Hintergrund zu rücken sowie von machtfixierten Beobachterpositionen abzuweichen. Auch die Einübung in neuartige (geregelte) Methoden, z.B. eines soziologischen Spiels, würde neue empirische Befunde ermöglichen und diesen auf einem qualitativ hohen Abstraktionsniveau in der Interpretation gerecht werden können.

Aus der vermeintlich dumm-seligen Werbung des AOL-Konzerns mit Boris Becker (,Ich bin drin!“) lassen sich demnach interessante raumtheoretische Fragestellungen extrahieren, die vor allem Formen von Raumartikulation betreffen, 
d.h. Fragen nach der kommunikativen Verfasstheit hochkomplexer Raumstrukturen. (Dr)innen \& dr(aussen)-Sein: Wo trinkt ein User mit einem anderen User Kaffee, wenn beide an verschiedenen Orten der Welt vor ihren Bildschirmen sitzen und sich über ihr Kaffeetrinken unterhalten? Im virtuellen Kaffeehaus? Oder wo verliebt man sich im Netz und hat gemeinsamen Sex? Dient der Monitor als Futon?

Die Frage nach der Virtualität von Realität angesichts der Realität virtueller Erfahrung stellt sich dem aufmerksamen User immer wieder. Vertiefende Analysen von Beobachterrollen und -strukturen würden eine Fortschreibung existierender Erkenntnismodelle fördern (vgl. Krämer 1996; Luhmann/Fuchs 1989; Metzinger 1999). Der Bildschirm, als optisches Portal zu einer imaginären Welt, dient vor diesem Hintergrund als Projektionsfläche (oder auch als Simulationsfläche) für reale (Probe-)Welten. Die Teilnahme an Kommunikationsformen, wie sie das Internet bietet, signalisiert von daher ein Handeln in Welten ohne eigenen Ursprung und dechiffriert eine soziologische Metaphysik, die ihren Sinn aus verlorengegangenen Objektbeziehungen gewinnt und neuerdings geosoziologisches Selbstbewusstsein im zeitgenössischen Mainstream kommuniziert.

Posten und Chatten am PC, das ist wie der intuitive Gebrauch eines Instrumentes, weshalb Musik, während eines Aufenthaltes in virtuellen Räumen, von besonderem Reiz sein kann. Die Vorstellung und Wahmehmung des Klangs reflektiert somit die Kunst der Spielerinnen und Spieler, sich im Raum zu positionieren; der Rest ist eine Frage der TechNick $(-)$

\section{DANKSAGUNG}

„Als ich zurücksah,

war die Welt ertrunken

in Kirschblüten."

(Chora, japanisches Haiku)

Wir(r) *ggg* danken herzlich allen unseren neuen Freundinnen und Freunden für die gemeinsam verbrachte Zeit im Netz und für die Forentreffen in Berlin/Dortmund/Wien! Ganz besondere Grüße gehen an: soulkitchen, mindbender, kinglear, springender_punkt, freie_meinung, cat \& maserati, crazy, dr yoghurt, hohes_gericht und Zitzenspitz. Was bleibt am Ende einer Untersuchung über nussbergvernarrte Yoghurts, vergessliche Flugnicks, metaphysische Threadpolizei, vergurkte Spreewalduhus und seelenbeerige Tintenaffen noch zu sagen? Es war der fisch art ige Spaß am Spiel und die rabe.laische Freude an beweglichen Regeln, die uns gaywichtig beyonerte \& verwienerte, denn: „Worum es im Netz geht, ist nicht, wer man ist oder vorgibt zu sein, sondern wie man sich erfinden kann. Was zählt, ist die Phantasie, ein überschäumender Geist, Ironie und Imagination. Es ist einerseits dieser ungeheure Überschuss, dieses enorme Potential, und andererseits die Art, wie sich alles bewegt, die Form, in der es erscheint" (Adamowsky 2000, S. 203, kursiv im Original). 


\section{LITERATUR \& MUSIK}

Adamowsky, Natascha (2000): Spielfiguren in virtuellen Welten, Frankfurt a.M.

Anderson, Laurie (1982): Big Science, Warner Bros, Records.

Bachtin, Michail M. und Rainer Grübel (2001): Die Ästhetik des Wortes, Frankfurt a.M.

Bollmann, Stefan und Christiane Heibach (Hrsg.) (1998): Kursbuch Internet, Hamburg.

Chick Corea (1972): Return to Forever, ECM Records.

Das weiße Rauschen (2002): Die Realität ist ein Hirngespinst, Normal Records.

Fischart, Johann (1997): Affentheuerlich Naupengeheurliche Geschichtsklitterung, Frankfurt a.M.

Foucault, Michel (1990): Andere Räume, in: Zeitmitschrift Ästhetik und Politik, Nr.1, Düsseldorf.

Funken, Christiane und Martina Löw (2002): Ego-Shooters Container. Raumkonstruktionen im elektronischen Netz, in: Maresch, Rudolf und Niels Werber (Hrsg.): Raum - Wissen Macht, Frankfurt a.M., S. 69-91.

Herz, J. C. (1996): Surfen auf dem Internet, Reinbek.

Kamper, Dietmar und Tom Fecht (Hrsg.) (2000): Umzug ins Offene. Vier Versuche über den Raum, Wien/New York.

Krämer, Sybille (1996): Bewußtsein. Philosophische Beiträge, Frankfurt a.M.

Luhmann, Niklas und Peter Fuchs (1998): Reden und Schweigen, Frankfurt a.M.

Maresch, Rudolf und Niels Werber (Hrsg.) (2002): Raum - Wissen - Macht, Frankfurt a.M.

Metzinger, Thomas (1999): Subjekt und Selbstmodell, Paderborn.

Rabelais, Francois (2003): Gargantua und Pantagruel, Frankfurt a.M.

Schlechter, Arne (2001): UTOPOLIS, in: Thabe, Sabine (Hrsg.): Raum und Sicherheit, Dortmund, S. 101-127.

Tanzwut (2000): Im Labyrinth der Sinne, EMI Electrola GmbH.

Tanzwut (2003): Ihr wolltet Spass, PICA RECORDS.

Thabe, Sabine (2000): Raum(de)konstruktionen. Reflexionen zu einer Philosophie des Raumes, Opladen.

Turkle, Sherry (1998): Leben im Netz. Identität in Zeiten des Internet, Reinbek.

Winnicott, Scott (1995): Vom Spiel zur Kreativität, Stuttgart. 



\title{
INTERNET, NICHT-ORTE UND DIE MIKROPHYSIK DES ORTES
}

\author{
Daniela Ahrens

\section{EINLEITUNG}

Die Schwierigkeiten bei der Bestimmung des Verhältnisses von Raum und Technik zeigen sich nicht zuletzt in den vielfältigen Beschreibungen und Metaphern hinsichtlich des Internets: Aus kultur- und medienwissenschaftlicher Perspektive wird das Internet erstens als virtueller Kultur- und Sozialraum verstanden, der neue Formen und Ausprägungen computervermittelter Identitätsbildung, der Interaktivität und Kommunikation ermöglicht. Der Blick richtet sich hier vornehmlich auf jene dialogischen Beziehungen, die bislang nur der mündlichen Kommunikation zwischen Personen vorbehalten blieben. Dialog- und Gemeinschaftsmetaphern wie „virtuelle Gemeinschaft“, „globales Dorf“, ,elektronische Gegenöffentlichkeit" u.a. deuten auf diese neue Situation hin. Unter der Bedingung der Gleichzeitigkeit entsteht eine Situation aktualer Reziprozität, d.h. die Teilnehmer können immer wieder ihre Sender/Empfänger-Position wechseln, ähnlich dem Sprecher-/Hörer-Wechsel. Durch die Möglichkeiten der wechselseitigen Übertragung von Informationen und den damit einhergehenden Interventions- und Gestaltungsmöglichkeiten der Teilnehmer in Echtzeit -- ohne dass die Nutzer denselben physischen Wahrnehmungskontext teilen - wird der bislang an Bedingungen der Kopräsenz gebundene Interaktionsbegriff auf den virtuellen Raum übertragen. Gerade weil der Interaktionsbegriff an bestimmte raumzeitliche Bedingungen geknüpft ist, verfuhrt er dazu, eine Bezugnahme auf den Raum zu konstatieren.

Mit dem Internet gewinnt zweitens die Netzmetaphorik an Konjunktur. Die Gleichzeitigkeit und Globalität des Netzes unterläuft die Integrität von Raum und Zeit in dem Sinne, dass Bewegungen im Raum nicht mehr gleichzeitig ein Fortschreiten der Zeit bedeuten. Im Netz ist alles gleichzeitig präsent und wir ,bewegen' uns in Sprüngen, folgen keinen richtungweisenden Wegen, sondern intuitiven und assoziativen Verknüpfungen. Der Netzbegriff verweist auf eine Verteiltheit im Raum, auf Beziehungen und Verknüpfungen, wobei die physische Verortung des Einzelnen für den Fortlauf der Kommunikation unerheblich ist. Von Bedeutung wird stattdessen die Frage des Angeschlossenseins. Es kommt zu einer „Ortsunabhängigkeit von Adressen“ (Stichweh 2000, S. 227): So verweisen zwar email-Adressen wie ,....uni.de“ noch auf eine institutionelle und nationale Zugehörigkeit, aber Adressen wie ,hotmail.com“ besitzen bereits keinerlei institutionelle oder räumliche Information mehr. Die Adressierung von Kommunikation 
geht nicht länger automatisch mit einer territorialen Lokalisierung einher. Adressen von Personen lassen sich immer weniger von Adressen von Computern unterscheiden. ${ }^{1}$

Drittens wird mit der Metapher der Datenautobahn, des Informationshighways, das Internet als Transportraum für Informationen verstanden. Das Internet, und hier insbesondere das World Wide Web, erscheint als reine Übertragungsleitung zum Vorteil und zur Annehmlichkeit des „Benutzers“, der als „Fahrer“ und Steuerer in einem straßenähnlichen Datenfluss navigiert. Hier wird der Eindruck erweckt, der Nutzen läge ausschließlich in der Schnelligkeit und Leistungsfähigkeit, eben mehr Texte, Bilder und Töne schneller und kostengünstiger zu übermitteln. Technik wird in erster Linie als Kontroll- und Disziplinierungstechnik verstanden, mit dem Ziel, Raum- und Zeitdifferenzen letztendlich zu eliminieren.

Alle drei auf das Internet bezogene Raummetaphern - virtueller Kommunikationsraum, Netz, Datenautobahn - rekurrieren zur Plausibilität ihrer Argumentation auf die Gegenüberstellung zwischen virtuellem und realen Raum. ${ }^{2}$ Der Gebrauch des Raumbegriffs zur Beschreibung der Besonderheiten einer neuen (Internet-)Geographie sollte uns jedoch nicht dazu verleiten, dem Internet bereits vorab bestimmte medienimmanente Raumqualitäten zu unterstellen. Zwar lässt sich beispielsweise in den virtuellen Umgebungen der MUD's und MOOs ${ }^{3}$ eine narrative Eigenräumlichkeit beobachten, in der die Teilnehmer die Räumlichkeit erzählerisch gestalten und programmieren (vgl. u.a. Sandbothe 1997; Funken/Löw 2002), dies jedoch als Anlass dafür zu nehmen, dass der Raum keine vorgegebene Entität darstellt, gerät allzu leicht in die Sogkraft virtueller Möglichkeiten. Begriffe wie Cyberspace und „virtual reality“ unterstützen den Trugschluss, es gäbe noch einen ,natürlichen“ Raum.

Der elektronische Raum ersetzt nichts - ist nicht prothetisch -, noch kann er als völlig losgelöst von dem uns bislang bekannten lokalen Nahraum gedacht werden. Es brauchte nicht das Internet, um aus soziologischer Perspektive die Begrenztheit eines am Containermodell orientierten Raumbegriffs aufzuzeigen. So wissen wir spätestens seit Simmel, dass die Grenze eine soziologische Tatsache ist, die sich räumlich formt. Das soziologische Interesse ist aufgefordert, sich

1 Kritik an dem „Körperverzicht“ der Netzperspektive formuliert Niels Werber. Am Beispiel der Folgen des 11. Septembers 2001 widerspricht Werber jenen Annahmen, die die Relevanz des physischen Raumes zugunsten eines losen gekoppelten Netzwerkes relativieren. Der Kampf gegen terroristische Netzwerke liefe ins Leere und bliebe ziellos, so lange lediglich die kommunikative Erreichbarkeit ins Visier genommen wird. Interventionen sind ebenso auf die genaue Lokalisierung nach Höhe und Breite angewiesen, wie die Verantwortlichen der Terroranschläge konkrete Ziele ausgesucht haben (vgl. Werber 2003).

2 Da im Rahmen dieses Beitrages keine ausführliche Auseinandersetzung hinsichtlich der Erklärungskraft der hier angesprochenen räumlichen Metaphern geleistet werden kann (vgl. hierzu u.a.: Ahrens 2001; Canzler u.a. 1995; Münker/Roesler 1997; Stefik 1996).

3 Abkürzung für Multi User Dungeon. In so genannten „virtuellen Spielhöllen“ loggen sich die Teilnehmer ein, um in einer fiktionalen textbasierten Spielelandschaft Punkte zu sammeln. MOO steht für Multi User Dungeon Object Oriented. Hierbei steht die Kreierung von Objekten und Räumen im Vordergrund. Jeder Spieler kann die Landschaft mitgestalten (vgl. Sandbothe 1997). 
weder von der Konkretheit räumlicher Gegebenheiten noch von der Stofflosigkeit virtueller Szenerien irritieren zu lassen.

Für den Beitrag leitend ist daher nicht die Unterscheidung zwischen ,virtueller Realität" und ,wirklicher Realität", denn bei der Internetnutzung wird keine Grenze in eine andere (begrenzte) Wirklichkeit überschritten. Vielmehr werden bisherige soziale Räume um einen neuen Möglichkeitshorizont angereichert. ${ }^{4}$ Es geht um die Kopplungen, Übergänge und Brüche: „It is time to relocate virtual culture in the real world“ (Robins 1995, S. 153). So entsteht ,mit der Installierung elektronischer Räume (...) die Notwendigkeit, das jeweils Lokale in seiner Beziehung zu globalen, grenzüberschreitenden Handlungsräumen neu zu bestimmen“" (Kompast/Wagner 1996, S. 279). Mit dem Verweis auf Kopplungen wird die Beobachterabhängigkeit von Raum herausgestellt, d.h. anstelle der Hypostasierung eines absoluten Raumes gewinnen Räume ihre Bedeutung durch die Kreuzung und Verknüpfung spezifischer Beobachtungsleistungen. Erst in dem jeweiligen Inbeziehungsetzen zwischen lokalem Nahraum und Internet wird der Raum aufgespannt, der genutzt wird. Hierbei ergeben sich neue Formen der Durchmischung globaler und lokaler Zusammenhänge, neue Spannungsfelder zwischen Abstraktheit und Kontextualität, zwischen Nähe und Ferne, zwischen Gleichzeitigkeit und Ungleichzeitigkeit. ${ }^{5}$ Legt man diese Beobachterperspektive zugrunde, erschließt sich die soziale Relevanz der ,Räumlichkeit des Raumes', bzw. der Mikrophysik des Ortes, durch die Art und Weise des kommunikativen Zugriffs. Räumliche Unterscheidungen wie nah/fern, hier/dort lassen sich demzufolge nicht als eindeutig messbare Maßstabsunterscheidungen begreifen, sondern als Unterscheidungen, auf die in der Kommunikation zurückgegriffen wird, um soziale Beziehungen zu ordnen und zu strukturieren. Das Verhältnis zwischen Raum und Sozialität aufgreifend fragt der Beitrag nach der Funktion des Raumes für soziale Beziehungen - und zwar jenseits der Unterscheidung von im Netz/außerhalb des Netzes bzw. online/offline.

Die These ist, dass die fortschreitende Enträumlichung von Kommunikation als Voraussetzung fungiert, auf den Raum bzw. auf räumliche Unterscheidungen zurückzugreifen, um auf diese Weise Komplexität bearbeitbar zu machen. ${ }^{6}$ Zur Entfaltung dieser These wird zunächst auf das Verhältnis zwischen sozialer Konstruiertheit und Gegenständlichkeit des Raumes eingegangen (2.). Die Radikalität, mit der das Internet soziale Beziehungen von räumlichen Gegebenheiten entkoppelt, wird anhand Faßlers Raumtypologie diskutiert (3.). Dass Faßler sein Raumverständnis aus den technischen Potenzialitäten des Internet ableitet, zeigt sich mit Blick auf Augés Konzept der Nicht-Orte. Obwohl (oder: gerade weil!) Augé selbst nicht explizit auf das Internet eingeht, lassen sich seine Überlegungen

4 Oder wie Paetau formuliert: „Virtualität als etwas Reales, als Vorhandenes ohne Dasein“ (Paetau 1997, S. 120).

5 Der Kontextbegriff verweist auf die jeweiligen sozialen und raumzeitlichen Umgebungsfaktoren, die als Erzeuger und Speicher von Unterschieden fungieren.

6 Dass die Einbeziehung des Raums keineswegs im Widerspruch zur Theorie der funktionalen Ausdifferenzierung steht, zeigt Nassehi am Beispiel des städtischen Raums und den dort sichtbaren sozialen Ungleichheiten (vgl. Nassehi 2002). 
nutzen, um zu verdeutlichen, dass der Raum an sich eigenschaftslos ist, dass der Raum erst durch die jeweiligen Anschlüsse und Beziehungen sozial relevant wird (4.). Abschließend werden die Überlegungen mit Blick auf die räumlichen Implikationen des Internets zusammengefasst (5.).

\section{ZWISCHEN KONSTRUIERTHEIT UND GEGENSTÄNDLICHKEIT}

Das gegenwärtige Interesse am Raum hat bislang weder in der Geographie noch in der Soziologie $\mathrm{zu}$ einer eindeutigen begrifflichen Bestimmung des sozialen Raumes geführt. Nach wie vor stellt sich die Frage, ob die Bezugnahme auf den Raum lediglich als eine Art „Zusatzargumentation“ (Brüsemeister 2002, S. 315) bei der Analyse sozialer Prozesse fungiert oder neben der sozialen, sachlichen und zeitlichen Dimension als zusätzliche Sinndimension in die Analyse mit einzubeziehen ist. Diese Verunsicherung gründet sich nicht zuletzt auf das Problem, ein Raumverständnis zu formulieren, das „eine Gleichwertigkeit und Aufeinanderbezogenheit von herausfordernder Materialität und hervorbringender Sozialität" (Sturm 2000, S. 145) berücksichtigt. Für die Soziologin Gabriele Sturm liegt hier eine der zentralen Aufgaben für ein zukünftiges Raumkonzept. Unterstellt wird dabei, dass der von uns wahrgenommene Raum als ein „gedoppeltes Phänomen“ zu begreifen ist, d.h. der Raum ist gleichermaßen ein materiales Gebilde und sozial indexikalisiert. Die Schwierigkeiten, die sich aus einem derartigen Raumverständnis ergeben, liegen in der Ausbalancierung zwischen Materialität und sozialer Konstruiertheit des Raumes. ${ }^{7}$ Kritiker verweisen dann je nach eigenem Forschungsinteresse auf die Materialität des Raumes, die sich einer kommunikativen (Ver-)Formung sperre, oder aber auf einen territorialen Fehlschluss durch eine Projektion sozialer Beziehungen auf den physischen Raum. ${ }^{8}$

Welche Rolle spielt die Materialität des Raumes, seine Gegenständlichkeit, wenn wir das Verhältnis zwischen elektronischem und realen Raum verwerfen zugunsten der Beobachterabhängigkeit von Raum? Die materielle räumliche Infrastruktur, die dem Internet in Form von Servern und Backbones zugrunde liegt, wird in der Nutzung invisibilisiert. Die Enträumlichung von Kommunikation, die Entkopplung von Sozialbeziehungen und physischem Raum hat mit dem Internet ihren vorläufigen Höhepunkt erreicht. Dass dennoch auf den Raum rekurriert wird, legt die Vermutung nahe, dass die Bezugnahme auf den Raum vielmehr aus Gründen der Herstellung von Ordnung und Orientierung erfolgt. Jenseits eines

7 Vgl. hierzu auch die Einleitung von Maresch/Werber und die dort aufgeworfenen Fragen nach der Beschaffenheit des Raumes (vgl. Marsch/Werber 2002, S. 13f.).

8 Vgl. hierzu u.a. aus sozialgeographischer Perspektive Klüters kommunikationsräumlichen Ansatz. Danach schafft sich jedes soziale System einen eigenen Systemraum. Die erdoberflächliche Bindung erfolgt zur Stabilisierung von sozialen Systemen (vgl. Klüter 1999, S. 195). Diese von Klüter als „Raumabstraktion“ (ebd., S. 193) begriffene Projektion sozialer Systeme auf die physische Umwelt erscheint jedoch problematisch, da territoriale Grenzen nicht als grenzbestimmend für soziale Systeme gedacht werden können (vgl. u.a. Stichweh 1998). 
territorialen Raumverständnisses symbolisiert der Raum Logik und „gedachte Ordnung“ (Reichert 1996, S. 17). Räumliches Denken hat etwas mit der Herstellung von Ordnung, der Handhabung mit Komplexität zu tun. Esposito zufolge besteht die ,interessanteste Eigenschaft eines raumbezogenen Denkens (...) in einer eigentümlichen Fähigkeit, konkrete Bestimmungen mit Kontingenz zu kombinieren“ (Esposito 2002, S. 36). Indem wir Reichweiten bestimmen, Grenzen sozial relevant werden lassen - etwa durch Zugehörigkeitsregeln - ordnen wir Menschen und Güter auf eine spezifische Weise, die aber - und hier kommen Prozesse der Verräumlichung bzw. des „Spacings“ (Löw 2001) ins Spiel - auch anders ausfallen können. Anders gesagt: Die Bezugnahme auf den Raum verweist auf Formen der Kontextualität.

Spätestens an dieser Stelle ist es an der Zeit, näher auf den Raumbegriff einzugehen. Drei Raumkonzepte lassen sich unterscheiden (vgl. Reichert 1996, Löw 2001): Erstens Raum als sichtbare, materielle Welt. Dieses chorische Raumverständnis findet sich im Alltag ebenso wie in der Wissenschaft. Zweitens Raum als ein real gegebenes unsichtbares Ding, das a priori unserer Wahrnehmung zugrunde liegt. Der Raum ist hier das vorgegebene „Wo“, innerhalb dessen beobachtbare Ereignisse stattfinden. Dieses Raumverständnis liegt dem Containermodell zugrunde, wonach der Raum als dreidimensionaler Behälter gedacht wird, der sich seinen Inhalten gegenüber indifferent verhält. Drittens Raum als logischer bzw. relationaler Raum. In diesem Raumverständnis hat der Raum keine vorgegebene Existenz, sondern entsteht durch die entdeckten und hergestellten Beziehungen von Menschen und Gütern zueinander. ${ }^{9}$

Der Beitrag knüpft an die dritte Bedeutung an, nach der die Konstituierung von Raum als relationaler Ordnungs- und Ortungsprozess zu verstehen ist. Löw spricht hier vom „Spacing", um die Prozesse der Platzierung und Positionierung kenntlich zu machen. Zwei Aspekte sind für die weitere Argumentation hervorzuheben: Zum einen geschehen die Prozesse der Verräumlichung, des „Spacings“ und der „Syntheseleistungen“, ${ }^{10}$ immer in bereits ,vorarrangierten Räumen“ (Löw 2001, S. 204). Zum anderen verweist der relationale Charakter von Raum auf die Zwischenörtlichkeit. Eine räumliche Perspektive einzunehmen, heißt demzufolge, die in der Kommunikation aufgespannte Differenz zwischen Regionen, Orten und Plätzen zu betrachten, um so beispielsweise regionale Besonderheiten und lokale Charakteristika zu identifizieren. Ein relationales Raumverständnis entledigt uns darüber hinaus von dem Dilemma, die Konstituierung von Räumen ausschließlich an das menschliche Handeln zu binden.

9 Den Übergang von der absoluten zur relativen Raumvorstellung formuliert von Weizsäcker in Abgrenzung zu Newtons absoluter Raumvorstellung: „Nachdem die nichteuklidische Geometrie gezeigt hat, dass verschiedene Strukturen des Raumes denkmöglich sind, liegt es nahe, mit der allgemeinen Relativitätstheorie anzunehmen, dass der Raum nicht eine fertige ,Mietska-

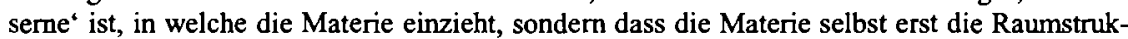
tur bestimmt“ (vgl. von Weizsäcker 1990, S. 149).

10 Durch Syntheseleistungen werden soziale Räume zu größeren Einheiten zusammengefasst. Die Syntheseleistung ,ermöglicht es, dass Ensembles sozialer Güter oder Menschen wie ein Element wahrgenommen, erinnert oder abstrahiert werden" (Löw 2001, S. 159). 


\section{ANWESEND/ABWESEND/FERNANWESEND}

Die Unterscheidung anwesend/abwesend fungiert als eine primäre Orientierung im Raum bei der Herstellung sozialer und räumlicher Distanzen. Dass sich dieses Begriffspaar angesichts der vielfältigen Möglichkeiten der Fern-Kommunikation mittlerweile als unterkomplex erweist, soll im Folgenden diskutiert werden. ${ }^{11}$ Inwiefern die technologische Entwicklung nicht nur die Überwindung von Raum in immer kürzeren Zeitintervallen ermöglicht und damit einer fortschreitenden Emanzipation vom Raum Rechnung trägt, sondern sich im Zuge dieser Entwicklung neue Räume aufspannen, zeigt Faßler anhand einer Typologie von „Raumschichtungen" (Faßler 1997, S. 76). Ausgehend von der körperzentrierten Raumerfahrung als „Urform“ sozialer Räume bilden sich im Zuge fortschreitender Technisierung immer abstraktere Räume aus, die sich zwar mit Hilfe von Technik konstituieren, jedoch nicht hinreichend über sie erklärbar sind.

- Körperliche Räume: Reichweiten bestimmen sich über die Position des Körpers, der als Nullpunkt des Koordinatensystems von Raum und Zeit fungiert. Körperliche Räume sind die Räume, die uns sinnlich verfügbar und in kurzer Zeit begehbar sind. ${ }^{12}$ Der Wahrnehmungsraum ist identisch mit dem sozialen Raum.

- Technische Räume: Hierunter fallen vielschichtige, geräte-, produktions-, medien- oder transporttechnische Aktions- und Wahrnehmungsräume sowie Wohn- und Arbeitsräume. Die Bedeutung körperzentrierter Nähe wird in diesen Räumen relativiert angesichts technisch gestützter Formen der Raumüberwindung. Raumerfahrung ist an mechanische Bewegung gekoppelt, da Bewegung - etwa mit der Eisenbahn, dem Auto - mit dem Körper erlebt wird: „Der körperliche Raum [wird] 'transportiert', versetzt" (Faßler 1997, S. 77).

- Mediale Räume: Mediale Räume bilden sich durch die Möglichkeit aus, Information und Kommunikation zu speichern. So werden durch die Flugschrift, das Buch, das Photopapier u.ä. Informationen zu anderen Orten transportiert, um dort neue Verbindungen herzustellen oder aber schon geknüpfte Sozialbeziehungen zu stabilisieren bzw. mit neuen Informationen zu versorgen. Die Reichweiten medialer Räume sind prinzipiell unbegrenzt, jedoch erfolgt die

11 Aus phänomenologischer Perspektive stellt der Körper die Referenz für die ,räumliche Aufschichtung der alltäglichen Lebenswelt“" (Schütz/Luckmann 1979, S. 63) dar. Die leibliche Präsenz bildet den Nullpunkt resp. den Ausgangspunkt für die Orientierung im Raum: „Relativ zu meinem Leib gruppiere ich die Elemente meiner Umgebung unter die Kategorien rechts, links, oben, unten, vorn, hinten, nah, fern usw.“ (ebd., S. 64). Diese räumliche Gliederung sozialen Handelns, in der soziale Nähe bzw. Distanz mit räumlichen Entfernungen korrespondiert, verliert zwar keineswegs an Plausibilität, sieht sich jedoch angesichts der Möglichkeiten neuer Technologien zunehmend der Konkurrenz neuer räumlicher Verteilungen und Anordnungen ausgesetzt, so dass neben dem Körper neue Referenzen abgestimmt werden müssen, um sich räumlich zu orientieren.

12 Hierzu gehören beispielsweise die von Goffman analysierten „Territorien des Selbst“ (Goffman 1974), die sich in Interaktionssituationen von Angesicht zu Angesicht ausbilden. 
Überwindung physischer Räume nicht in Echtzeit. Kommunikation erfolgt zeitversetzt.

- Elektronische Räume: Mit dem Einsatz von Elektrizität ermöglichen Telegraphen bzw. später das Telefon, Nachrichten über Impulse und elektronische Intervalle über weite Entfernungen hinweg schnell zu übertragen. Gegenüber den mitunter großen Zeitdehnungen der mechanischen Speichermedien wird eine direkte Beantwortbarkeit möglich. Mit der Ausbildung elektronischer Räume ist der erste wichtige Schritt für Fernanwesenheit vollzogen, und die Erfahrung gleichzeitigen Sprechens und Zuhörens wird Bestandteil einer neuen Kommunikationskultur.

- Kybernetische soziale Räume: Faßler zielt hier auf das Internet ab. Mit dem Einsatz globaler Vernetzungstechnologien entstehen virtuelle Räume, ,computational environments" (ebd.), die ebenso nahe, regionale wie auch kontinentale und globale Räume bedeuten können. In diesen Räumen kommt es zu einer Loslösung von territorialen, körperlichen oder gegenständlichen Bindungen. In diesen fluiden, ,unfassbaren“ Räumen büßen territoriale, institutionelle und körperliche Referenzmuster an Orientierungsqualität ein.

Folgt man Faßler, führt die Dehnung und Ausweitung des engen Interaktionsund Wahrnehmungsraumes mittels Technik zu immer abstrakter werdenden Räumen. So wie im Raum Orte mit spezifischen Bedeutungen aufgeladen werden, differenzieren sich aufbauend auf elektronischen Schaltungszuständen neue soziale Räume aus, deren raumzeitliche Stabilität jedoch mit der kommunikativen Nutzung steht und fällt. Faßler spricht denn auch in diesem Zusammenhang nicht von ortlosen, sondern von "territoriumsfreien Gesellschaften“ (Faßler 2001, S. 96), die aus Nutzungs-'Orten' bestehen. Während die moderne Gesellschaft durch formale, taylorisierte Umgebungen geprägt war, haben wir es jetzt angesichts netztechnischer Reichweiten mit verteilten, dynamischen Räumen zu tun. Mit Blick auf die raumrelevante Unterscheidung Nähe/Ferne wird deutlich, dass sich der Wahmehmungsraum nicht mehr vom Nullpunkt des Körpers in konzentrischen Kreisen ausdehnt. Entgegen der These der „Vernichtung des Raumes“ entstehen mediale Räume in der kommunikativen Hervorbringung. Diese elektronischen Kommunikationsumgebungen bedürfen angesichts ihrer sozialen, räumlichen und zeitlichen Indeterminiertheit der permanenten Stabilisierung und Vergewisserung. Durch den Verlust des physischen Raumes als messbares, begehund erfahrbares Prüfkriterium für Wirklichkeitsaussagen muss die Kommunikation auf räumliche Unterscheidung zurückgreifen, um für sich einen verstehbaren Kontext zu schaffen. Elektronische Anwesenheit kennzeichnet sich durch einen erhöhten Regelungsbedarf, denn indem das „erkennbare Gesicht“ (ebd., S. 191) verschwindet, gehen auch nonverbale Kommunikationsformen verloren. Die „Spur eines Lächelns“, der „Anflug von Wut", die „Augen-Blicke“ geraten aus dem Gesichtskreis. ${ }^{13}$ In der medialen Präsenz wird es somit notwendig, sich und

13 In diesem Zusammenhang lassen sich jedoch Entwicklungen beobachten, die versuchen, die „Natürlichkeit" direkter Interaktion in den elektronischen Raum zu übertragen. Um das virtu- 
sein Gegenüber permanent aufs Neue zu situieren und zu kontrollieren, um eine eigene Kommunikationsumgebung auszubilden. Während also in der face-to-faceInteraktion die Interaktionssituation sicher ist, weiß man in den computertechnisch generierten Umgebungen nie genau, inwieweit der Gesprächspartner nicht gleichzeitig Nebengespräche führt oder andere Dinge tut. Diese Unsicherheit geht soweit, dass wir gar nicht genau wissen können, mit wem bzw. ob wir überhaupt mit einer Person oder mit einem besonders cleveren Computerprogramm kommunizieren. Gegenüber Formen kopräsenter Kommunikation ist die computergestützte Kommunikation darauf angewiesen, diesen Raum als einen Kontext der Orientierung erst zu erzeugen. „Dem elektronischen Raum fehlt das Bezugssystem, von dem aus beobachtet und Geschwindigkeit, Beschleunigung, Nähe und Distanz erlebt werden können" (Wagner 1993, S. 30). Die Kommunikationsteilnehmer müssen sich erst in dem Kommunikationsraum „einschließen“. „Wie der Teilnehmer immer im Kontext der Interaktion eingeschlossen ist, so ist der Cybernaut im Cyberspace eingeschlossen“ (Esposito 2002, S. 45). Als Folge dieser Ausdifferenzierung unterschiedlicher Realitätsebenen tritt neben die Differenz von anwesend/abwesend eine weitere Differenz hinzu, und zwar die zwischen Anwesenheit und Fern-Anwesenheit. So unterstützen elektronische Vernetzungstechnologien die Tendenzen der Auflösung der Bedeutung des lokalen Zusammenhangs für die Entstehung von Sozialität, gleichzeitig jedoch verdichten sich die zusätzlichen Erlebnis-, Wahrnehmungs- und Interaktionsräume zu „glokalen“ Handlungsarenen, in denen sich neue Formen der Organisierung globaler und lokaler Bezüge ausbilden.

Es geht um eine Neujustierung räumlicher Begrifflichkeiten, und zwar um die „sozio-kulturelle Neufassung von Ferne, und damit des Anderen und des Fremden" (Faßler 1996, S. 181). Die Raumbezüge sozialen Handelns haben sich insofern gewandelt, dass nichts mehr ohne Ferne geht, dass wir neue „Routinen der Ferne“ (Faßler 1997, S. 27) ausbilden müssen und infolgedessen eine „sozialtheoretische Eroberung der Fern-Anwesenheit" (ebd., S. 199) notwendig wird. Wenn Entfernung immer weniger als Ausgrenzung gedacht werden kann, noch sich ausschließlich messen lässt, dann wird es notwendig, „Ferne und Anonymität als

elle Interaktionsgeschehen möglichst „natürlich" erscheinen zu lassen, werden so genannte „Talking Heads“, sprechende Köpfe, entwickelt. Bei der Modellierung der virtuellen Interaktion nach dem Vorbild der face-to-face Interaktion wird das über ein Hochgeschwindigkeitsnetz übertragene Bild des jeweiligen Gesprächspartners in Form einer „digitalen Fototapete“ auf eine Büste projiziert, so dass der Eindruck physischer Anwesenheit entsteht. Auf diese Weise will man virtuelle Konferenzen plastischer machen und eine größere Annäherung an die „natürliche Gesprächsatmosphäre" schaffen. Diese Form der holographischen Darstellung stellt darauf ab, die nonverbale Kommunikation - Gestik, Mimik etc. - in virtuellen Interaktionen sichtbar zu machen, denn ,auch das Augenzwinkern soll ankommen“ (Sperlich 1997). Das Interface rückt durch diese Form der Telepräsenz zunehmend in den Hintergrund. Es wird unsichtbar. Die Gesprächsteilnehmer sitzen nicht mehr vor einem Monitor, sondern agieren im virtuellen Raum als Teleakteure. Im Zuge einer derartigen Rekonfiguration des Körpers im virtuellen Raum verwischt die Grenze zwischen physischer und virtueller Präsenz. Virtuelle Repräsentanten erhalten den Status eigenständiger Akteure, die es immer schwieriger werden lassen, noch eindeutig festzulegen, was (schon) technisch ist und was (noch) menschlich. 
konstitutive Voraussetzung“ (ebd., S. 194) anzuerkennen, wenn es um das Verständnis von Identität und Gesellschaft geht. Ferne mischt sich als eine ,agierende Ferne" (ebd., S. 197) ein. Vor dem Hintergrund dieser Entwicklungen kann der Begriff der Ferne immer weniger als substantialistische (territoriale oder körperliche) Begrifflichkeit gedacht werden. Wir haben es jetzt vielmehr mit einer ,informationellen Ferne“ (Faßler 1996, S. 189) zu tun, die zu „einem Wissens-, Gesprächs-, Informations-Zustand geworden ist" (ebd.). Ferne verliert ihren territorialen Bezug und wird als ,informationelle Ferne" (ebd.) zur neuen Zustandsbedingung für Interaktions- und Kommunikationsverhältnisse. Es kommt im Zuge dieser Entwicklung zu einer grundlegenden Umkehrung: Nicht vom Mittelpunkt des Körpers - wird Anwesenheit als sinnlich erreichbare oder symbolisch bekannte Umgebung gedacht, sondern „Ferne schreibt sich durch ihre 'Nutzung' material und in kürzester Handlungszeit in Nähe ein" (Faßler 1996, S. 188).

Faßlers Raumtypologie gründet sich auf eine technikzentrierte Perspektive, in deren Vordergrund die Neuordnung des „Nexus von Körper und Umwelt" steht (Faßler 1997, S. 68). Als „pluridimensionales“ Konstrukt kann der Raum nur unter Absehung seiner Vieldeutigkeit auf eine eindeutige räumliche Unterscheidung - nah/fern, geschlossen/offen - reduziert werden. Anders gesagt: Die Vieldeutigkeit des Raumes legt die Vermutung nahe, dass wir nicht mit ein und demselben Begriff von Raum für alle Arten des Sozialen arbeiten können. Dass sich der enge körperzentrierte Raumbegriff der Interaktion ab einer gewissen Stufe mediengestützter Kommunikation als nutzlos, wenn nicht gar als sinnlos, erweist, heißt nicht, dass sich dadurch das Problem des Räumlichen erledigt. ${ }^{14}$ Die abnehmende Relevanz geographischer Entfernungen für Sozialbeziehungen ist nicht zwangsläufig mit einem Obsoletwerden des Raumes verbunden. „Das ,Wo' der Kommunikation lässt sich nicht anzeigen in dem Sinne, dass sie kein Körper mit angebbaren Raumkoordinaten ist. Das ,Wo' der Kommunikation lässt sich jedoch anzeigen in dem Sinne, dass wir mit gutem Recht sagen: dieses Gespräch (cobodily presence) hat etwa in der Universitätsbibliothek, und jenes (telefonisch) in der Stadt Freiburg stattgefunden, wobei die Ereignisse der Kommunikation in beiden Fällen durch die besagten Umstände mitbestimmt wurden" (Filippov 1999, S. 349). Es stellt sich dann aber die Frage, wie es dazu kommt, dass mit der Verbreitung und wissenschaftlichen Analyse des Internets Bezugnahmen auf den Raum an Konjunktur gewinnen.

Um dieser Frage nachzugehen, soll im folgenden Abschnitt am Beispiel von Augés Konzept der „Nicht-Orte“ deutlich gemacht werden, inwiefern sich durch die Enträumlichung sozialer Beziehungen neue Funktionen des Raumes ergeben.

14 Schreibt man allein den kopräsenten Interaktionsformen eine Relevanz des Raumes zu, wird implizit an einem Containermodell von Raum festgehalten. 


\section{DAS INTERNET ALS VERTEILERRAUM}

Mit seiner These, dass die „Übermoderne“ Nicht-Orte hervorbringt, geht es Augé um die Bedeutungsverschiebung gesellschaftlicher Raumerfahrungen (Augé 1994, S. 38) ${ }^{15}$ Befand sich das Forschungsobjekt der Anthropologen bislang in erster Linie im konkreten Hier und Jetzt, in der Untersuchung der in Raum und Zeit eindeutig lokalisierbaren Kultur, werden sie jetzt zunehmend mit einer neuen Form des Raumes konfrontiert, und zwar mit „Nicht-Orten“. Nicht-Orte entstehen aus der paradoxen Situation heraus, dass die gegenwärtigen weltweiten Vernetzungsmöglichkeiten nicht nur zu einem Schrumpfen räumlicher Entfernungen und einer „Verkleinerung von Welt" führen, vielmehr konfrontieren sie uns gleichzeitig mit einem „Übermaß an Raum“ (Augé 1994, S. 40). Nicht-Orte stellen nichtsymboliserte Orte dar, die sich nur durch geringe spezifische Eigenschaften kennzeichnen. In den Nicht-Orten verlieren Orte ihre Einzigartigkeit, ihre spezifischen, regionalen Besonderheiten, mithin ihre Authentizität. Es kommt zu einer ,artifiziellen Authentizität". So lassen sich Feriendörfer und Hotelketten ebenso wie Durchgangswohnheime und Flüchtlingslager als Nicht-Orte begreifen. Es sind Orte, die sich nicht durch geschlossene Sinnwelten kennzeichnen, sondern sich mit ihren Aufforderungen - etwa Hinweistafeln, Vorschriften, Verbote etc. - an jeden richten: „Den wirklichen Nicht-Orten der Übermoderne, an denen wir uns befinden, wenn wir über die Autobahn fahren, in einem Supermarkt einkaufen oder in einem Flughafen auf den nächsten Flug nach London oder Marseille warten, ist es eigen, dass sie auch von den Worten oder Texten definiert werden, die sie uns darbieten " (ebd., S. 113). Nicht-Orte sind in gewissem Sinne identitätslos. Es sind „Transiträume“ (Augé 1994, S. 93; vgl. auch Gumbrecht 1991, S. 58) bzw. „Verteilerräume“, die zahlreiche, je unterschiedliche Anschlüsse an mögliche Räume und Zeiten bieten. Als Transiträume verweisen Nicht-Orte auf eine Welt der Durchreise, des Provisorischen und Ephemeren. Durch ihre soziale Indifferenz sind es Orte zufälliger Begegnung. Angesichts zunehmender Mobilität, steigenden Migrationsströmen und einer expandierenden Tourismusindustrie sieht Augé in diesen Nicht-Orten das „,Maß unserer Zeit, das sich quantifizieren lässt" (Augé 1994, S. 94). Sie bilden die Summe aus den Flugstrecken, Bahnlinien, Autobahnen, Flughäfen, Einkaufszentren und ähnlichem und seit jüngster Zeit auch der virtuellen Netze. Kennzeichnet sich der qualitativ aufgeladene symbolische Ort durch die Realität der festen Wohnung, der unmittelbaren Sozialbeziehungen mithin der physischen und sozialen Nähe und unmittelbaren Wahr-

15 Mit dem Begriff „Übermoderne“ setzt Augé den Schwerpunkt auf das „Übermaß“, das die gegenwärtige Entwicklung kennzeichnet. Danach haben wir es in zeitlicher Hinsicht mit einer „mit Ereignissen überladenen Zeit“ (Augé 1994, S. 39) zu tun, in räumlicher Hinsicht mit einer „Überfiulle des Raumes“ und als dritte Figur des Übermaßes nennt Augé das Ich bzw. die Zunahme individueller Sinnkonstruktionen (vgl. ebd., S. 38f.). 
nehmung, dominieren in den Nicht-Orten die Realitäten des Transits. Im Vordergrund steht die Reise als spezifischer Umgang mit dem Raum. ${ }^{16}$

Nicht-Orte sind indifferent gegenüber Sozialität. Dies heißt jedoch nicht, Nicht-Orte wären für die Strukturierung sozialer Beziehungen bedeutungslos. Im Gegenteil: Gerade die Indifferenz, die Entkopplung von Sozialem und NichtOrten fungiert als Voraussetzung dafür, auf die Raum-Kategorie zurückzugreifen, um Sozialbeziehungen zu strukturieren, um eine Begrenzung von Eindrücken zu erreichen.

Überträgt man Augés Überlegungen zu Nicht-Orten auf die hier im Vordergrund stehenden Überlegungen zum Internet, lassen sich Rückschlüsse auf die Funktion des Internets ziehen. Neben den Aspekten der Homogenität und Standardisierung ist hier Augés Verständnis von Nicht-Orten als Transit- und Verteilerräumen hervorzuheben. Der erweiterte Rahmen weltweiter Information und Kommunikation ist mit einer unübersehbaren Menge von Unwägbarkeiten und Überraschungen versehen, so dass der Informationscharakter der Netzkommunikation hoch situativ bleibt. Bei der Internetnutzung erhalten Zufälle und Unvorhersehbares einen neuen Stellenwert. Es ergeben sich Schnittpunkte und „Begegnungen", die eine unter physischen Bedingungen nur schwer herzustellende Spontaneität aufweisen. Das Internet fungiert auf der Zeichenebene als Verteilerraum. Als anonymer, homogener und hochstandardisierter elektronischer Raum liefert das Internet einen Variationsspielraum und zwingt zur Selektion. Diese Selektion erfolgt nach sachlichen und sozialen Kriterien. Die soziale Relevanz des Raumes ergibt sich nicht bereits aus seinem Vorhandensein als Punkt auf der geographischen Landkarte oder - mit Blick auf das Internet - durch die Adresse einer Homepage, sondern dadurch, dass dies und nicht jenes gewählt wird und so weitere Entscheidungen vorstrukturiert werden. Durch die Selektion wird ein gewisses $\mathrm{Ma} \beta$ an Überschaubarkeit und Bestimmtheit erreicht, das Voraussetzung für aktives Handeln ist.

Während Augés kulturpessimistische Einschätzung - Nicht-Orte erzeugen als identitätslose Orte Einsamkeit und Anonymität - das Soziale durch die Räumlichkeit determiniert und beeinflussbar versteht, soll hier nach der besonderen Funktion von Nicht-Orten als Transit- oder Verteilerräumen gefragt werden. Ausgehend davon, dass ,erfahrungsimprägnierte' und identitätsstiftende Orte und abstrakte, homogene Räume eine Seite der Beobachtung darstellen, ${ }^{17}$ geht es um die Frage nach den je verschiedenen Funktionen der Ordnungsbildung. Überlegungen,

16 Da für diesen Zusammenhang Verräumlichungsprozesse als Prozesse des (An-)Ordnens verstanden werden, spielt es an dieser Stelle keine Rolle, ob wir es mit virtuellen Datenreisen zu tun haben oder mit Flugreisen.

17 „So wie die anthropologischen Orte Organisch-Soziales hervorbringen, so schaffen die NichtOrte eine solitäre Verträglichkeit" (Augé 1994, S. 111). Das Abstraktwerden des Raumes wird von Augé als Bedrohung und als schleichender Verlust von Individualität und Authentizität verstanden. In seiner Kritik an der Artifizialität des Raumes gerät ihm aus dem Blick, dass auch die von ihm als authentisch gekennzeichneten Orte künstliche Grenzen aufweisen und Wege und Richtungen immer weniger über natürliche Grenzen (Gebirgsverläufe, Flüsse u.ä.) ,wegweisend' sind. 
inwieweit räumliche Unterscheidungen für soziale Beziehungen relevant sind, müssen beides im Blick haben: Zum einen die Virtualisierung räumlicher Entfernungen sowie die mediale Repräsentation geographischer Orte, zum anderen den konkreten lokalen Ort und die physische Nähe. Als Knotenpunkte, die unterschiedliche Anschlussmöglichkeiten bieten, liefern Nicht-Orte die Voraussetzung dafür, den konkreten lokalen Aufenthaltsort als einen von vielen möglichen Orten wahrzunehmen. Indem Nicht-Orte als Mittler fungieren, verweisen sie auf den Horizont des Möglichen und die permanente Notwendigkeit, Eindrücke und Beobachtungen zu begrenzen.

Nicht-Orte sind sozial hergestellte Räume, die selbst nicht durch soziales Handeln geprägt werden. Von Nicht-Orten gehen Prozesse des Spacings aus, wobei Nicht-Orte genutzt werden, ohne dass diese Nutzung sich in die Nicht-Orte auf Dauer einzuschreiben vermag. Thre Funktion liegt darin, einen Überschuss an Möglichkeiten zu bieten. Verteilerräume erfordern somit ein raumbezogenes Denken, d.h. die Herstellung von Kontextualität. Sie machen die Beobachterabhängigkeit von Verräumlichungsprozessen sichtbar, indem sie deutlich werden lassen, dass jede kontextuell realisierte Wirklichkeit mit anderen konkurriert und umgekehrt gerade in ihrer Kontextualität der Verfuggbarkeit von anderen Kontexten her Grenzen setzt. Kontextualität ist nur in Abgrenzung oder in Kontrast zu anderen möglichen und präsenten Kontexten zu haben. Formen räumlicher Ansässigkeit, von Infrastrukturen oder Objekten und deren Bedeutungen haben somit keinen Maßstab an sich, sondern sind ,verhältnismäßig'. Es entsteht eine Vielzahl verschiedenartiger räumlicher Ordnungen.

\section{FAZIT}

Zwei Möglichkeiten, den Raum neu zu denken, sind hier vorgestellt worden. Die eine Möglichkeit ist Faßlers an die Technikentwicklung gebundene Auffächerung von Raumtypologien. Unter medientechnologischen Voraussetzungen unterstreicht Faßler die Bedeutung ,informationeller Ferne“, verankert sein Raumverständnis in der Cybermoderne, in der Server, Backbones und Schaltungsgeschwindigkeiten als neue postgeographische Raummaße fungieren. Indem die elektronischen Schaltungszustände als wegweisend für neue Raumformen gedacht werden, verbleibt der Raum jedoch in der Elektronik verankert und wird als rein technische Umgebungsbedingung für neue kulturelle Phänomene gedacht (vgl. u.a. Faßler 1996). Auf der anderen Seite - ausgehend vom konkreten lokalen Ort - lenkt Augé den Blick auf die Zunahme von Nicht-Orten, die als Verteilerräume fungieren. Durch sein Festhalten an der vergemeinschaftenden Funktion des konkreten authentischen Ortes werden die Verteilerräume jedoch in erster Linie als Bedrohung für das Lokale verstanden. Derartige Verengungen versucht das relationale Raumverständnis zu überwinden, indem es die aufgespannten Beziehungen zwischen den unterschiedlichen Räumen in den Blick rückt. Die Praxis der Relationierung wird zu einer notwendigen Methode, Komplexität in den Griff zu bekommen und so die soziale Wirklichkeit verstehbar zu machen. Indem Rela- 
tionen auf die Perspektivenabhängigkeit von Raumkonstituierungen abstellen, bilden sie Formen „nicht-beliebiger Kontingenz" (Esposito 2002, S. 38) aus. Entgegen der Unterscheidung virtuell versus real betont das relationale Raumverständnis die Relevanz des Netzes, ohne jedoch den Netzgedanken allein an die Technik zu binden. Demzufolge lässt sich nicht mehr bereits vorweg bestimmen, ob das Lokale der Ort ist, von dem aus abstrakte, distante Räume verstanden und interpretiert werden, oder ob das Internet das Lokale ,entdeckt'. Dies wird erst in der Relationierung entschieden. Online und Offline, hier und dort, verlieren ihre eindeutige räumliche Verankerung und treten als eigenständig markierte Bereiche hervor. Das Außen wird nicht als das Unbestimmte gedacht, sondern als das Außen, dass dadurch generiert wird, dass man ein Innen schafft. Die Herstellung von Bezügen und Verbindungen sowie das Abgrenzen und Schützen spezifischer Räume und Perspektiven erfordert ein hohes Maß an (Kommunikations-)Arbeit. Gegenüber dem modernen Container-Raum wird der Raum hier nicht als Rahmenbedingung, sondern als soziales Konstrukt gedacht, das aufs engste mit den jeweiligen Formen der Interaktion und Kommunikation verwoben ist. Inwiefern in diesem Zusammenhang durch den Einsatz des Internets Veränderungen des Lokalen als eines Kommunikationssystems entstehen, scheint insofern ein aufschlussreiches Forschungsprogramm gleichermaßen für Soziologen und Geographen.

Abschließend ist festzuhalten, dass das Internet nicht zu einer Virtualisierung und Auflösung räumlicher Differenzen führt - es sei denn, man hält an einem ontologischen Raumbegriff fest. Anstelle eines Obsoletwerdens des Raumes lassen sich Strategien der Neutralisierung, der Invisibilisierung und der Kontingenzeindämmung sowie der Begrenzung von Eindrücken beobachten. Sichtbar werden diese neuen Formen, wenn es gelingt, ein territoriales Raumkonzept hinter sich zu lassen und das Containermodell zu verabschieden. Weder wird der körpergebundene lokale Raum zum phantasmagorischen Ort, noch beschert uns das Internet eine neue Nomadologie. Auch unter globalisierten Bedingungen ,lebt niemand in der Welt im Allgemeinen“ (Geertz 1996, S. 262).

\section{LITERATUR}

Ahrens, Daniela (2000): Grenzen der Enträumlichung. Weltstädte, Cyberspace und transnationale Räume in der globalisierten Modeme, Opladen.

Augé, Marc (1994): Orte und Nicht-Orte.Vorüberlegungen zu einer Ethnologie der Einsamkeit, Frankfurt a.M.

Brisemeister, Thomas (2000): Zwischen Welt und Dorf - Raum als Differenzienungsprinzip von Gegenwartsgesellschaften, in: Volkmann, Ute und Uwe Schimank (Hrsg.): Soziologische Gegenwartsdiagnosen II, Opladen, S. 315-340.

Canzler, Weert u.a. (1995): Die Datenautobahn - Sinn und Unsinn einer populären Metapher. WZB-Paper, FS II, Berlin, S. 95-101.

Esposito, Elena (2002): Virtualisierung und Divination. Formen der Räumlichkeit der Kommunikation, in: Maresch, Rudolf und Niels Werber (Hrsg.): Raum - Wissen - Macht, Frankfurt a.M., S. 33-49. 
Faßler, Manfred (1996): Privilegien der Ferne. Elektronische Landschaften, transkulturelle Kommunikation und Weltrhetorik, in: ders., J. Will und M. Zimmermann (Hrsg.): Gegen die Restauration der Geopolitik. Zum Verhältnis von Ethnie, Nation und Globalität, Gießen, S. 166-203.

Faßler, Manfred (1997): Was ist Kommunikation? München.

Faßler, Manfred (2000): Netzwerke: Einführung in die Netzstrukturen, Netzkulturen und verteilte Gesellschaftlichkeit, München.

Filippov, Alexander (1999): Der Raum der Systeme und die großen Reiche. Über die Vieldeutigkeit des Raumes in der Soziologie, in: Honegger, Claudia u.a. (Hrsg.): Grenzenlose Gesellschaft? Verhandlungen des 29. Kongresses der Deutschen Gesellschaft für Soziologie, des 16. Kongresses der Österreichischen Gesellschaft für Soziologie, des 11. Kongresses der Schweizerischen Gesellschaft für Soziologie in Freiburg i. Br., Opladen, S. 344-357.

Funken, Christiane und Marina Löw (2000): Ego-Shooters Container. Raumkonstruktionen im elektronischen Netz, in: Maresch, Rudolf und Niels Werber (Hrsg.): Raum - Wissen Macht, Frankfurt a.M., S. 69-92.

Geertz, Clifford (1996): Welt in Stücken. Kultur und Politik am Ende des 20. Jahrhunderts, Wien.

Goffman, Erving (1974): Das Individuum im öffentlichen Austausch: Mikrostudien zur öffentlichen Ordnung, Frankfurt a.M.

Gumbrecht, Hans Ulrich (1991): nachMODERNE ZEITENräume, in: Weimann, R. u.a. (Hrsg.): Postmoderne - globale Differenz, Frankfurt a.M., S. 54-73.

Klüter, Helmut (1999): Raum und Organisation, in: Meusburger, Peter (Hrsg.): Handlungszentrierte Sozialgeographie. Benno Werlens Entwurf in kritischer Diskussion, Stuttgart, S. 187-212.

Kompast, Martin und Ina Wagner (1996): Der Computer als Architekturlabor. Zur widersprüchlichen Modernisierung professioneller Praktiken und Identitäten, in: Flecker, J. und J. Hofbauer (Hrsg.): Vernetzung und Vereinnahmung. Arbeit zwischen Internationalisierung und neuen Managementkonzepten. Österreichische Zeitschrift für Soziologie, Sonderband 3, Opladen, S. 275-303.

Löw, Martina (2001): Raumsoziologie, Frankfurt a.M.

Maresch, Rudolf und Niels Werber (Hrsg.) (2002): Raum - Wissen - Macht, Frankfurt a.M.

Münker, Stefan und Alexander Roesler (Hrsg.) (1997): Mythos Internet, Frankfurt a.M.

Nassehi, Armin (2002): Dichte Räume. Städte als Synchronisations- und Inklusionsmaschinen, in: Löw, Martina (Hrsg.): Differenzierungen des Städtischen, Opladen, S. 211-233.

Paetau, Michael (1997): Sozialität in virtuellen Räumen? in: ders. und Barbara Becker (Hrsg.): Virtualisierung des Sozialen. Die Informationsgesellschaft zwischen Fragmentierung und Globalisierung, Frankfurt a.M./New York, S. 103-134

Reichert, Dagmar (1996): Räumliches Denken als Ordnen der Dinge, in: ders. (Hrsg.): Räumliches Denken, Zürich, S. 15-43.

Sandbothe, Mike (1997): Interaktivität - Hypertextualität - Transversalität. Eine medienphilosophische Analyse des Internet, in: Münker, Stefan und Alexander Roesler (Hrsg.): Mythos Internet, Frankfurt a.M., S. 56-83.

Schütz, Alfred und Thomas Luckmann (1997): Strukturen der Lebenswelt, Bd. 1, Frankfurt a.M.

Sperlich, Thomas (1997): Auch das Augenzwinkern soll ankommen, in: Sonntagszeitung vom 18.05.1997.

Stefik, M. (1996): Introduction, in: ders. (Hrsg.); Internet Dreams, Archetypes, Myths and Metaphors, Cambridge, S. 6-14. 
Stichweh, Rudolf (1998): Raum, Region und Stadt in der Systemtheorie, in: Soziale Systeme, Jg. 4, Heft 2, S. 341-358.

Stichweh, Rudolf (2000): Adresse und Lokalisierung in einem globalen Kommunikationssystem, in: ders. (Hrsg.): Die Weltgesellschaft. Soziologische Analysen, Frankfurt a.M., S. 220-231.

Sturm, Gabriele (2000): Wege zum Raum. Methodologische Annäherung an ein Basiskonzept der Raumpplanung, Opladen.

Wagner, Ina (1993): Neue Reflexivität. Technisch vermittelte Handlungs-Realitäten in Organisationen, in: ders. (Hrsg.): Kooperative Medien. Informationstechnische Gestaltung moderner Organisationen, Frankfurt a.M.New York, S. 7-66.

Weizsäcker, Carl F. von (1990): Zum Weltbild der Physik, Stuttgart.

Werber, Niels (2003): Die Medien der Weltgesellschaft, online: http://homepage.ruhr-uni-bochum.de/niels.werber/Medien-Koeln.html

Werlen, Benno (1993): Gibt es eine Geographie ohne Raum? Zum Verhältnis von traditioneller Geographie und zeitgenössischen Gesellschaften, in: Erdkunde. Archiv für wissenschaftliche Geographie, Jg. 47, Heft 4, S. 241-254. 



\title{
ZWISCHEN DEN WELTEN \\ RAUMSIMULATIONEN IM NETZ
}

\author{
Detlef Kanwischer
}

\section{SOFTWARE FÜR KOPFRÄUME}

„Software für Massaker" titelte die FAZ-Sonntagszeitung am 28. April 2002, nachdem Robert Steinhäuser im Erfurter Gutenberg-Gymnasium 16 Lehrer, 2 Schüler und sich selbst erschossen hatte. Das Computerspiel Counterstrike wurde von den Medien aufgrund einiger Analogien als hauptverantwortlich für den Amoklauf von Steinhäuser gemacht. Zwischen Simulation und Alltagsleben lag bei Steinhäuser - im Computerspieler-Jargon ausgedrückt - womöglich nur ein "Level“. Hatte Steinhäuser das Töten am Rechner gelernt? Hatte er das Töten als Spiel gesehen? Wenn dem so wäre, dann müssten schon längst - bei 250000 regelmäßigen Counterstrike-Spielern in Deutschland - ganze Landstriche entvölkert sein. Die Kollision von virtuellen Welten und Alltagsleben ist so einfach nicht zu erklären. Jürgen Fritz, der als einer der profiliertesten Experten seit über zehn Jahren die Wirkungen von Computerspielen analysiert, hebt hervor: „Man muss ein Geflecht von verschiedenen Verstärkern und Ursachenfolgen betrachten. Und wenn auf diesem Feld dramatische Missverhältnisse entstehen, kommt es zum Amoklauf. Die Eindeutigkeit, das Computerspiel hätte ihn dazu gebracht, lehne ich ab“ (Krempl 2002).

Im Internet werden schon seit Jahren Online-Spiele angeboten. Während die erste Spielegeneration noch textbasiert war, kamen in den letzten Jahren zunehmend graphische Spiele auf den Markt. Die bekanntesten sind die Ego-ShooterSpiele (so genannt, weil sie aus der Ich-Perspektive gespielt werden) wie Counterstrike und Half-Life. Neben diesen Spielen kann man heutzutage aber auch Simulations- und Strategiespiele wie Anno 2003 online spielen. Demnächst geht auch Sim City, eines der bekanntesten Simulationsspiele, online. Die Hersteller von Spielekonsolen haben zudem ihre Produkte onlinefähig gemacht. Ziel ist es, dass nicht mehr gegen den Computer gespielt wird, sondern gegen Spieler aus aller Welt - „alles, was man dazu braucht, ist eine Sega Dreamcast-Spielekonsole, ein Kabel zur Telefonbuchse, ein Fernseher und los geht die Schlittenfahrt durch die Welt des Internets" (Werbeanzeige).

Roman Herzog hat schon 1999 darauf hingewiesen, „dass die Schulen im Unterricht die durch die Technik grundlegend veränderte Lebenswirklichkeit aufgreifen". Diese grundlegend veränderte Lebenswirklichkeit wird v.a. durch die neuen Virtuellen Welten geprägt. „Virtuell“ bedeutet so viel wie „der Kraft oder Möglichkeit nach vorhanden". Virtuelle Welten sind also der Kraft oder Möglichkeit 
nach vorhandene Welten. Aber gab es denn dann nicht schon immer virtuelle Welten? Die meisten von uns erschaffen sich in Träumen andere Welten. Film und Fernsehen vermitteln Scheinwelten. Die Freizeitindustrie schafft künstliche Ferienwelten. Piloten lernen an Flugsimulatoren, ein Flugzeug zu steuern. Menschen halluzinieren unter dem Einfluss von Drogen. Andere wiederum erfahren virtuelle Welten während einer Messe oder anderen liturgischen Feiern. Soldaten üben an Computerspielen des Typs Ego-Shooter das Töten. Die Werbung inszeniert ständig virtuelle Welten; trinken wir Barcardi, weil er uns schmeckt oder weil uns ein Schluck in das Paradies des Lasziven versetzt? Lange noch könnte diese Aufzählung weitergehen. Immer schon gab es virtuelle Welten. Seit Platon's Höhlengleichnis füllen Abhandlungen über die damit einhergehenden Wirklichkeitsvorstellungen ganze Bibliotheken. Durch die Ereignisse von Erfurt und Littleton wird jedoch die Frage nach den Veränderungen unserer Wirklichkeitsvorstellungen durch die digitale Virtualisierung mit neuer Schärfe aufgeworfen und auch in der breiten Öffentlichkeit diskutiert.

Auch die raumbezogenen Sozialwissenschaften haben die Thematik der digitalen Virtualisierung aufgegriffen. Neben dem Aspekt des "death of distance“ werden vor allem die Konstruktionen und Funktionen von digitalen virtuellen Räumen untersucht. Ein Schwerpunkt der Fragestellungen bezüglich der Anbieterseite zielt darauf ab, ob die virtuellen Räume als uneinheitlich, offen und multiperspektivisch oder als einheitlich umgebende Behälter konstruiert werden (vgl. Funken/Löw 2002). Auf der Nachfragerseite wird untersucht, wie der virtuelle Raum von den Nutzern inszeniert und angeeignet wird und inwiefern eine gegenseitige Beeinflussung des virtuellen und des realen Raumes stattfindet (vgl. Hülsmann 2000 und Schroer 2001).

Im Zuge der Digitalisierung des Lernens haben sich auch im Geographieunterricht die Computersimulationsspiele ihren Platz erobert. Der Einsatz von Spielen wie Hunger in Afrika, Stadtplanung Karbach, Ecopolicy und Mobility ist heutzutage üblich. Diese Simulationsspiele werden zwar noch nicht online gespielt, aber es wird nicht mehr lange dauern, bis auch dies möglich ist. Mobility steht zumindest schon als "download“ bereit. Die Computersimulationsspiele dienen im Unterricht vorrangig dem Erlernen von bestimmten Wissensgebieten. Die (Raum-)Konstruktionen dieser virtuellen Welten und die damit einhergehenden Wirklichkeitsvorstellungen der Schüler(innen) werden jedoch bisher nicht weiter hinterfragt.

Ich werde daher in diesem Beitrag anhand der Computersimulation Mobility und dem Medienwirkungsmodell von Jürgen Fritz (1997) den Fragen nachgehen, mit welchem geographischen Raumverständnis hier gelehrt wird und welchen Einfluss dies auf die Wirklichkeitsvorstellungen von Schüler(inne)n haben kann. Dazu wird im Folgenden zunächst aufzeigt, welche Erwartungen mit dem Einsatz von Computersimulationen im Unterricht üblicherweise verbunden werden (2.). In Abgrenzung von der herkömmlichen Art und Weise, den Erfolg von Computersimulationen im Unterricht nur im Hinblick auf die erreichten Lerneffekte zu untersuchen, werden alternative Fragen zur Erforschung ihrer Wirkung formuliert. Im Anschluss daran werde ich nach einer kurzen Vorstellung von Mobility (3.) ge- 
nauer darauf eingehen, welche Raummodelle dieser Simulation implizit unterliegen und durch sie idealtypischerweise - im interaktiven Prozess des Spielens vermittelt werden (4.). Zur Analyse ihrer tatsächlichen Wirkung auf Schüler(inne)n eignet sich Fritz' Medienwirkungsmodell „Transfer und Transformation" (5.). Mit seiner Hilfe lassen sich das durch Computersimulationen erzeugte Verstrickungsverhältnis zwischen virtueller und realer Welt sowie die damit einhergehenden Wirklichkeitsvorstellungen von Schüler(inne)n untersuchen. Dies wird exemplarisch am Beispiel eines Interviews mit einer Schülerin, die Mobility gespielt hat, demonstriert (6.). Abschließend werde ich die sichtbar gewordenen Probleme des Einsatzes von Computersimulationsspielen im (Geographie-) Unterricht diskutieren (7.).

\section{COMPUTERSIMULATIONEN UND WIRKLICHKEITSVORSTELLUNGEN IM GEOGRAPHIEUNTERRICHT}

Schon seit Ende der 1980er Jahre liegen Computersimulationen für den Geographieunterricht vor. Ein Blick in die geographiedidaktischen Zeitschriften der letzten Jahre verdeutlicht, dass Computersimulationen offensichtlich zunehmend in das Unterrichtsgeschehen integriert werden. Hierbei werden nahezu alle Themenbereiche abgedeckt. Das Spektrum der vorliegenden Simulationsprogramme reicht vom Landschafts- und Klimawandel über Mobilitätsmodelle bis hin zur Stadtplanung. Hinsichtlich der didaktischen Überlegungen zum Einsatz von Computersimulationen werden verschiedene Vorteile und Gefahren diskutiert (vgl. Hemmer 1997, S. 218f.):

\section{Vorteile}

- Erleichterung des Denkens in vernetzten Systemen

- Verdeutlichung der Konsequenzen monokausalen Denkens

- Erwerb kybernetischen Wissens durch das Betreiben dynamischer Systeme

- Einsicht in komplexe Systeme und Erwerb von Kenntnissen über Ursachen, Verläufe und Folgen von Problemen komplexer Systeme (z.B. Umweltprobleme)

- Einsicht in die Wirkung der Veränderung eines Systemparameters, besonders in den Fällen, in denen die Wirkungen nicht von vornherein erfassbar sind

\section{Gefahren}

- zu starke Vereinfachung realer Systeme und zu starke Reduktion der wirksamen Parameter mit der Gefahr, zu einem bloßen, oft utopischen Spiel zu werden, das den Blick auf die Wirklichkeit eher verstellt, als sie zu erhellen

- unkritische Übertragbarkeit der fiktiven Programmaussagen auf entsprechende Wirklichkeitsbereiche

- Suggestion der Mathematisierbarkeit von Gesellschafts-Umwelt-Problemen 
- Ausklammerung von historischen, kulturellen, sozialen und politischen Einflüssen

- Ausblendung der individuellen Bedürfnisstruktur und des Entwicklungsstandes des Lernenden

Die Auflistung der Vorteile und Gefahren verdeutlicht, dass Computersimulationen nicht nur der Informations- und Wissensvermittlung dienen, sondern dass die Schüler(innen) im Rahmen einer Unterrichtsstunde, in der eine Computersimulation eingesetzt wird, auch eine virtuelle Welt betreten, darin handeln und sie wieder verlassen.

Die geographiedidaktische Forschung zum Thema Computersimulation im Unterricht befasst sich aber bisher ausschließlich mit den Lerneffekten bei Schüler(inne)n. Eine sogenannte Medienwirkungsforschung wird überraschenderweise noch nicht praktiziert. Dazu wären die in dem Medium Computersimulation durch die Anbieter (d.h. die Autoren und Entwickler) konstruierten virtuellen Räume sowie die Wirkungen dieser Räume auf die Nachfrager (die Schüler) in den Mittelpunkt der Analyse zu rücken. Ebenso wenig Berücksichtigung finden in der einschlägigen Forschung bislang Fragestellungen zu den komplexen Beziehungen zwischen Schüler(inne)n und Computersimulation (z.B.: „Welche Konstruktion von Raum und Wirklichkeit nehmen die Schüler(innen) mit in die virtuelle Welt hinein und welche wieder mit hinaus?", „Welche Eindrücke aus der Computersimulation werden in die reale Welt transformiert und welchen Veränderungen sind sie ausgesetzt?").

\section{DAS COMPUTERSIMULATIONSSPIEL MOBLITY}

„Planen und bauen Sie Ihre eigene Stadt und managen Sie dort die Mobilität Ihrer Einwohner. Gestalten Sie für Ihre Bürgerinnen und Bürger ein attraktives und zugleich wirtschaflich wie auch ökologisch lebensfähiges Umfeld. Das ist MOBILLITY - das Verkehrssimulationsspiel!" (www.mobility-online.de)

Mobility wurde im Auftrag der Daimler-Chrysler AG und der Verkehrsverbunde Rhein-Ruhr und Rhein-Main unter wissenschaftlicher Begleitung der BauhausUniversität Weimar sowie der Verkehrsforschung der Daimler-Chrysler AG entwickelt und vom Bundesministerium für Bildung und Forschung gefördert. Maßnahmen zur Beeinflussung des Verkehrs, Raumplanung und Umwelteinwirkungen werden in dieser Computersimulation über Verfahren umgesetzt, die auf den professionellen Modellen von Raum- und Verkehrsplanern basieren.

Erste Erfahrungen mit Mobility im Schuleinsatz wurden mit Hilfe von eigens für das Programm erstellten Unterrichtsmaterialien für Lehrer(innen) gesammelt. Diese Materialien vertiefen Einzelaspekte des Themas Verkehr. Für die ausgehende Mittel- und die Oberstufe sind vier Themengruppen (Verkehrsmittelvergleich, Stadt und Verkehr, Stadtmodelle sowie Verkehrsplanung und Zukunftsszenarien) entwickelt worden. Sie berühren die natur-, technik- und sozialwissen- 
schaftlichen Aufgabenfelder in der Schule und sind in viele kleine Einzelbausteine unterteilt, die man im Unterricht flexibel kombinieren kann.

Den Herausgebern zufolge sind von den Programmierern ca. 150 Variablen zu einem komplexen, wechselwirkenden Netz versponnen worden. Welche Variablen dies im einzelnen sind und wie deren Wechselwirkungen genau modelliert wurden, ist leider nicht dokumentiert.

\section{DIE RÄUMLICHKEIT IN DER HANDLUNGSWELT VON MOBILITY}

Die Arbeitsgruppe „Curriculum 2000+ der Deutschen Gesellschaft für Geographie" geht in ihren Grundsätzen und Empfehlungen für die Lehrplanarbeit im Schulfach Geographie davon aus, dass „Räumlichkeit über Lebens- und Handlungswelten existentiell erfahren wird". Unter dieser Prämisse werden vier Typen von „Räumen“ unterschieden (vgl. Arbeitsgruppe 2002, S. 5):

- Erstens werden „Räume“ im „realistischen“ Sinne als Container aufgefasst, in denen bestimmte Sachverhalte der physisch-materiellen Welt enthalten sind.

- Zweitens werden „Räume“ als Systeme von Lagebeziehungen materieller Objekte verstanden.

- Drittens gilt „Raum“ als eine Kategorie der Sinneswahrnehmung und damit als „Anschauungsform“, mit deren Hilfe Individuen und Institutionen ihre Wahrnehmung ordnen und so die Welt in ihren Handlungen ,räumlich“ differenzieren.

- Das bedingt, dass „Räume“ viertens auch unter der Perspektive ihrer sozialen, technischen und gesellschaftlichen Konstruiertheit betrachtet werden müssen, indem danach gefragt wird, ,wer unter welchen Bedingungen und aus welchen Interesse wie über bestimmte Räume kommuniziert und sie durch alltägliches Handeln fortlaufend produziert und reproduziert".

Vor diesem definitorisch-konzeptionellen Hintergrund stellt sich im Zusammenhang von Mobility die Frage: Welches Verständnis von Raum bzw. welche Perspektive auf Raum wird den Schüler(inne)n in der Handlungswelt der Computersimulation vermittelt?

Mobility bedient sich zunächst einer inselartigen Konstruktion. Die zu bebauende Fläche ist als grünes Wiesenland dargestellt und nach außen von einem Blau begrenzt, über das man keine Verfügungsgewalt hat. Der Spiel-Raum, innerhalb dessen die Spieler ihre Stadt errichten, ist also klar abgegrenzt. Ein mit der Stadt in Korrespondenz stehendes Umland und regionale, nationale oder internationale Verflechtungen der Stadt werden nicht berücksichtigt. Das dem Simulationsspiel zugrunde liegende Raummodell entspricht folglich dem einfachen Containermodell. Aber schauen wir uns an, welche räumlichen Perspektiven sich innerhalb des Containers realisieren lassen. 
Während des Spielgeschehens können die Spieler Wegstrecken (Neben-, Haupt- und vierspurige Straßen sowie Stadtbahnen) bauen und Bebauungsflächen (Wohn-, Büro- und Industriegebiete) ausweisen, die mit verkehrsrelevanten Gebäuden (Einkaufszentren, Schulen, Theater, Kinos, Sportstätten oder Parkanlagen) und verkehrstechnischen Gebäuden (Carsharing-Verwaltungsgebäude, Bereitstellungsflächen für Carsharing-Autos, Sendemasten, Mobilitätszentralen und Parkplätze) bebaut werden können. Hierbei liegt der Akzent besonders auf der Bedeutung von Standorten, Lage-Relationen und Distanzen. Neben einer Schule muss z.B. stets eine Haltestelle des ÖPNV gebaut werden. Einkaufszentren und Bürogebiete erfordern die Ausweisung von Parkplätzen. Industriegebiete sollen, wegen der erhöhten Emissionswerte, wenigsten vier Felder von benachbarten Wohngebieten entfernt errichtet werden. Sportstätten dienen zwar dem Wohlbefinden der Bevölkerung, sie sollen aber wegen der Lärmbelästigung nicht in Wohngebieten liegen. Der Raum wird hierbei also aus der Perspektive der Lagebeziehungen materieller Objekte betrachtet. Keine Rücksicht wird jedoch darauf genommen, wie diese Lagebeziehungen von den in der Stadt lebenden Individuen, Gruppen oder Institutionen wahrgenommen werden und in ihre Lebens- und Handlungspraxis hineinreichen. Die Relevanz von „Raum“ als einer Kategorie der Sinneswahrnehmung wird unterschlagen.

Neben diesen bebauungstechnischen und rechtlichen Aspekten gibt es noch eine Reihe von Funktionsdifferenzierungen, mit deren Hilfe die Spieler ihre Stadt organisieren können. So sind die Verkehrsdienstleister unterteilt in alternative Verkehrskonzepte und ÖPNV. Die alternativen Verkehrskonzepte wiederum beinhalten einen Personal Travel Assistant, eine Mobilitätszentrale, eine passive und eine aktive Zielführung, Carsharing und Werbung. Der ÖPNV dagegen wird durch die Einzelfunktionen Finanzen, Fuhrpark, Linienführung, Kundendienst, Personal, Werbung und Fahrpreise unterschieden. Als wichtigste Steuerungsfunktion der Rubrik Parkraummanagement ist die Gebührenzuweisung vorgesehen. Und mit der Stadtverwaltung schließlich können die Spieler die Stadtentwicklung über die Funktionen Finanzen, Forschung, Gesetze, Steuern und $\mathrm{Zu}$ schüsse beeinflussen.

Trotz der Differenzierung all dieser Funktionen erreicht Mobility nicht annähernd die Pluralität der Wirklichkeit. Durch die Stadtverwaltung kann der Spieler z.B. den Stadtbewohnern Zuschüsse für die Anschaffung eines Computers für Telearbeit zukommen lassen. Die dahinter stehende Logik zielt darauf ab, dass mehr Heimarbeitsplätze entstehen und somit der Berufspendlerverkehr abnimmt. Die Rolle der arbeitnehmer- und arbeitgeberspezifischen Einstellungen zur Telearbeit wird jedoch vollkommen außer Acht gelassen. Auch die Werbung für ÖPNV und alternative Verkehrskonzepte erfolgt pauschalisierend und nicht differenziert nach Nachfragergruppen. Das Handeln der unterschiedlichen Akteure (Investoren, Stadtbewohner, Politiker) und ihr Eingebundensein in standortspezifische, rechtliche und symbolische Strukturen wird nicht thematisiert. Die komplexe Wechselwirkung zwischen gesellschaftlichen Strukturen und sozialen Handlungen, mit all ihren räumlichen Beschränkungen und Auswirkungen, taucht 
nur monoperspektivisch aus der Sicht des Stadtplaners (bzw. des Programmierers) auf - nicht auch aus der Sicht der betroffenen Stadtbewohner.

Zusammenfassend lässt sich festhalten, dass die Computersimulation Mobility nur die Perspektive des Containerraumes und die darin bestehenden Lagebeziehungen materieller Objekte berücksichtigt, d.h. nur zwei der vier von der Arbeitsgruppe "Curriculum 2000+“ unterschiedenen Raumtypen. „Raum" wird somit lediglich als eine in der gesellschaftlichen Realität vorhandene, den Handlungen von Individuen, Gruppen und Institutionen aber vorgängige Größe aufgefasst. Die Einsicht, dass Raum als eine Wahrnehmungsform auch multiperspektivisch vorkommt und damit ganz unterschiedlich relevant wird, bleibt ebenso folgenlos für die Gestaltung der Simulation wie die Einsicht, dass Räume auch soziale Räume sind, die im Handlungs- und Kommunikationsprozess sozial hergestellt, reproduziert und verändert werden. Obwohl die Raumkategorie im Alltagsleben als sowohl materiell wie auch sozial konstituiert erfahren wird, begnügt sich Mobility mit der Verwendung eines behälterförmigen Raums, in dem sich die für die Spieler relevanten Lagestrukturen und Weltdeutungen befinden.

Für die Lehrerin (den Lehrer) ergibt sich hieraus die Frage, ob die aktuellen gesellschaftlichen Probleme mit solch' einer reduktionistischen Perspektive auf Raum überhaupt aufgegriffen werden können. Wenn die Einbettung der Computersimulation in den Unterricht den aktuellen didaktischen Standards entsprechenden soll, sollte sie/er außerdem wissen, welchen denkbaren Einfluss das dem Spiel unterliegende Raumverständnis auf die Wirklichkeitsvorstellungen von Schüler(inne)n hat.

\section{5. ÜBERLEGUNGEN ZU EINEM WIRKUNGSMODELL DER VIRTUELLEN WELT}

Geht es um die Welt der Computerspiele, dann verbreiten die Medien nicht selten alarmierende Meldungen darüber, was die Nutzung mit dem Nutzer macht (s. das eingangs beschriebene Beispiel des Amoklaufs von Robert Steinhäuser in Erfurt). Aber ebenso wie im Falle von Film und Fernsehen wird das öffentliche Bedürfnis nach klaren und orientierenden Einschätzungen der möglicherweise schädigenden Wirkung dieser Medien von den Medienwirkungsforschern „nur“ mit einem differenzierenden „Es kommt drauf an" beantwortet; gesicherte Ergebnisse zur Wirkung von Film, Fernsehen und Computerspielen gibt es zur Zeit nicht.

Die Informationen der Medien werden nicht ungefiltert zum Anwender übertragen. Dieser selegiert und konstruiert sich vielmehr die für ihn relevanten Informationen vor dem Hintergrund seiner individuellen Lebenserfahrungen. Das Problem der empirisch kaum zu kontrollierenden individuellen Nutzereigenschaften betrifft auch Forschungen, die die Wechselwirkungen von virtuellen Welten und dem „realen“ Leben der Anwender untersuchen. Es gibt zwar eine Fülle von Einzeluntersuchungen, insbesondere zum Thema „Virtuelle Welt und reale Gewalt", aber ihnen fehlt ein modellorientiertes Forschungsparadigma, das die Austausch- und Transformationsprozesse vor, während und nach dem Aufenthalt in 
einer virtuellen Welt angemessen wiedergibt. Das Sozialpädagoge Jürgen Fritz (1997) hat ein solches Modell entwickelt, das ich im Folgenden skizzieren werde.

Das Modell geht von der Annahme aus, dass sich unsere Wahrnehmung nach unterschiedlichen Lebenswelten differenziert - der realen, mentalen, medialen und virtuellen Welt sowie der Traumwelt. Zwischen diesen Welten nimmt Fritz Transferprozesse an: „Transfer ist (...) die Übertragung des Gelernten auf eine andere Aufgabe oder eine andere Situation" (Fritz 1997, S. 229). Bei diesen Transferprozessen werden Erfahrungen allerdings mit bereits bestehenden individuellen Strukturen und Schemata auf unterschiedlichen Ebenen des Bewusstsein verglichen. Fritz stellt daher heraus, dass ,das Paradigma von Ursache und Wirkung bei der Beurteilung intermondialer Transferprozesse unzulänglich und die Diskussion um die Wirkung der medialen und virtuellen Welten in der realen Welt vom Ansatz her problematisch ist. Es kommt vielmehr darauf an zu prüfen, auf welchen Ebenen der Transfer stattfindet und wie der Angleichungsprozess zwischen Reizeindruck und Schemata verläuft" (ebd., S. 231). Fritz unterscheidet in seinem Modell „Transfer und Transformation“ zwischen fünf unterschiedlichen Transferebenen:

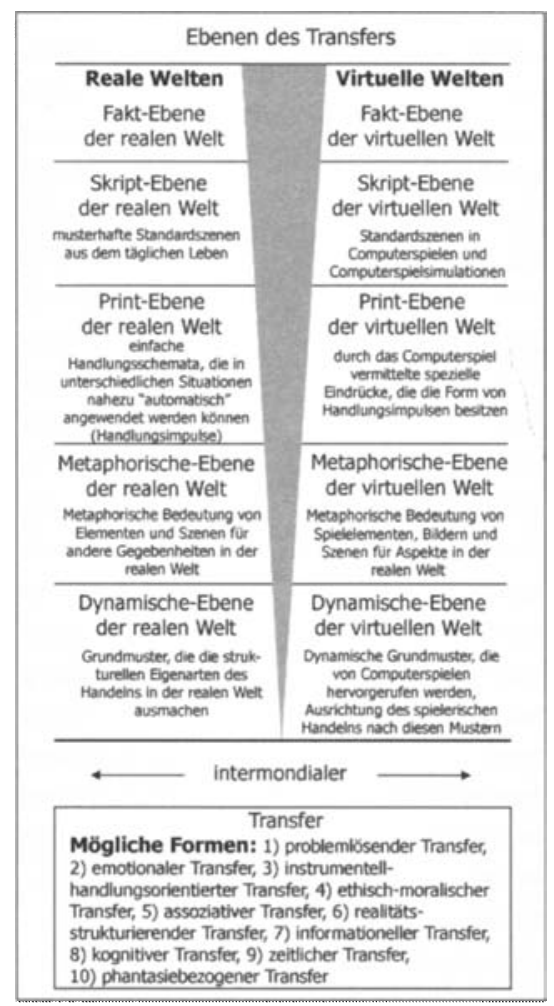

Abbildung 1: Das Wirkungsmodell „Transfer und Transformation" von Jürgen Fritz (Quelle: Fritz 1997, S. 237). 
Auf der Fakt-Ebene geht es um konkrete Tatsachen bzw. Informationen, die für die reale Welt bedeutsam sein können. Bei Mobility werden z.B. Sachinformationen zu alternativen Verkehrskonzepten und den Verwaltungsbereichen einer Stadt bereit gestellt. Skripts sind Schemata für bestimmte Ereignisabläufe bzw. musterhafte Standardszenen, die einen inhaltlichen und sozialen Bezug haben. Diese mentalen Drehbücher helfen uns, bestimmte Handlungen auszuführen. Klassische Beispiele sind der Restaurantbesuch oder das Benutzen von öffentlichen Verkehirsmitteln. Unser Verhalten in diesen Situationen folgt bestimmten Regeln, Erwartungen und Standards. Die zu einem Skript zusammengefassten Handlungsketten lassen sich besonders gut auf ähnliche Situationen übertragen. Skripte werden nicht nur von der virtuellen in die reale Welt transferiert, sondern, wie das Rollenspiel zeigt, auch von der realen in die Spielwelt. Bei diesen intermondialen Transfers ändert sich zwar nicht die Struktur der Skripts, wohl aber ihre lebensweltbezogene Bedeutung, ihr „Rahmen“. Einfache Handlungsmuster wie ,in die Hände klatschen“, ,auf einem Bein hüpfen“, „einen Ball wegwerfen“, „eine Tür öffnen“, die nur eine begrenzte Handlungstiefe und geringe kontextuelle Verankerung besitzen, werden Prints genannt. Im Gegensatz zum Skript verfügt der Print nur über einfache Handlungsabfolgen und ist losgelöst von sozialen Bezügen und Kontexten. Während Transfers auf der Skript-Ebene relativ konkrete Handlungsschemata zugrunde liegen, erfolgt der metaphorischen Transfer in einem wesentlich abstrakteren Modus. Transferiert werden keine der realen Welt entnommenen Informationen oder Skripts, denen das unmittelbare Erleben anhaftet. Es sind lediglich symbolisch-funktionale Schemata und Bedeutungszusammenhänge (z.B.: „das ist ja wie bei Mobility"), die übertragen werden. Auf der dynamischen Ebene schließlich bezieht sich der Transfer nicht mehr auf einzelne Szenen, sondern ist losgelöst von allen inhaltlichen Bezügen. Hier geht es um die Frage der medialen „Botschaft“ des Computerspiels. Bei Mobility wäre dies z.B. die intakte Umwelt, bei vielen Ego-Shooter-Spielen geht es um Macht und Kontrolle. Fritz geht davon aus, dass, je tiefer die Ebene ist, desto unbewusster die Transfers sind. Den einzelnen Ebenen des Transfers stellt er zehn mögliche Formen des Transfers gegenüber (ebd., S. 232ff.).

Für den reflektierten Umgang mit Computersimulationen im Unterricht stellt sich nun für den (die) Lehrer(in) die Frage nach dem Verstrickungsverhältnis zwischen realer und virtueller Welt: Auf welchen Ebenen kann es bei den Schüler(inne)n zu einem Transferprozess zwischen virtueller und realer Welt kommen? Dass es bisher noch keine gesicherten Erkenntnisse zur Wirkung von Computerspielen gibt, liegt nicht zuletzt darin begründet, dass von einem vielschichtigen Wirkungsgeflecht ausgegangen werden muss, das bei der Auswertung entsprechenden Datenmaterials gerade wegen seiner Komplexität immer auch eine Vielzahl von Lesarten zulässt. Im Folgenden möchte ich am Beispiel der Schülerin Farina eine solche Lesart einer möglichen Transferleistung vorstellen. 


\section{6. „DAS IST JA GAR NICHT SO WIE BEI UNS!“}

Die Schülerin, mit der ich diesen kleinen Versuch durchgeführt habe, heißt Farina, wohnt in einer Großstadt, ist 15 Jahre alt, besucht das Gymnasium im Übergang zur 10. Klasse und ist eine besonders erfolgreiche Schülerin. In ihrer Familie gibt es einen Computer, der von der gesamten Familie genutzt wird. Sie nutzt den Computer alle zwei bis drei Tage für E-mails, zur Internetrecherche, Textverarbeitung und zum Spielen. Farina spielt Denk- und Strategiespiele am Rechner. Beim Spielen achtet die Mutter darauf, dass Farina nicht länger als ein bis zwei Stunden am Tag spielt. In der Schule ist der Einsatz des Computers von den jeweiligen Lehrer(inne)n abhängig. Bei manchen Lehrer(inne)n wird der Computer häufig genutzt, bei manchen nur unregelmäßig und bei anderen gar nicht. Simulationsspiele hat Farina bisher in der Schule noch nicht gespielt.

Ich habe Farina das Spiel Mobility auf ihren Computer installiert. Anschließend haben wir uns gemeinsam das Tutorial angeschaut und ich habe ihr noch ein paar wichtige Funktionen gezeigt. Da sie Ferien hatte, sind wir so verblieben, dass sie je nach Zeit und Lust Mobility spielen konnte. Nach zwei Tagen habe ich dann ein Gespräch mit ihr über das Spiel geführt. Insgesamt hat Farina sechs Stunden Mobility gespielt. In dieser Zeit hat sie unterschiedliche Städte gebaut und verschiedene Szenarien ausprobiert.

In dem Gespräch habe ich Farina eingangs um eine generelle Stellungnahme zu dem Spiel gebeten:

Farina: Es ist auch wieder so ein Suchtspiel, das man immer weiter machen möchte, um die Stadt zu entwickeln. Aber ich finde es ein bisschen unübersichtlich, wie man die ganzen Sachen bedient, mit den Buslinien habe ich unheimlich viel Zeit gebraucht, bis ich das herausbekommen habe. Aber was ich gut finde, ist, dass einem immer angezeigt wird, was man machen soll. Woher soll ich denn auch wissen, was eine Stadt braucht, kann ich ja nicht wissen. Viele Sachen habe ich aber noch nicht ausprobiert, z.B. das mit den Steuern, wie man die verändern kann.

In dieser Aussage wird deutlich, dass der Motivationseffekt bei Farina sehr hoch war. Deutlich wird aber auch, wie abhängig Farina von den Programmgestaltern des Spiels ist. Da sie der Ansicht ist, dass sie nicht weiß, was eine Stadt alles braucht, vertraut sie den kleinen Textfeldern, die regelmäßig auf dem Bildschirm auftauchen, um ihr aufzuzeigen, was sie als nächstes machen soll. Thre Handlungen wurden also mehr oder weniger fremdgesteuert.

Im weiteren Verlauf des Gesprächs haben wir uns dann über die im Spiel aufgetretenen Probleme und Zusammenhänge des Themas Stadtplanung und Mobilität unterhalten:

D.K.: Welche Probleme bei dem Thema Mobilität bzw. Stadtplanung sind aufgetaucht?

Farina: Meine Busse waren dauernd überlastet, ich sollte immer neue Linien einrichten, kürzere Taktzeiten, das war ein bisschen extrem. 
D.K.: Wie hast Du dieses Problem gelöst?

Farina: Das war einfach, da richtest du halt neue Linien ein oder verkürzt die Taktzeiten.

D.K.: Welche Zusammenhänge hast Du entdeckt bei dem Thema Mobilität und Stadtplanung?

Farina: Die müssen halt von den Wohngebieten zu Schulen und zur Innenstadt kommen, da muss man immer Buslinien einrichten. Wenn man Buslinien im Industriegebiet hat, dann werden die kaum genutzt.

D.K.: Glaubst Du, dass sich die verwendeten Problemlösungsstrategien auch in die Realität übertragen lassen?

Farina: Ja klar, das ist ja schon so, dass in der Wirklichkeit die Buslinien auch so gebaut werden, dass man vom Wohngebiet in die Innenstadt kommt.

Diese Gesprächssequenz zeigt auf, dass die Vorteile von Computersimulationen (z.B. die Einsicht in die Wirkung bei der Veränderung eines Systemparameters oder der Erwerb kybernetischen Wissens) auch bei Mobility zum Tragen kommen. In Bezug auf das Wirkungsmodell „Transfer und Transformation“ von Jürgen Fritz wird deutlich, dass bei Farina auf der Fakt-Ebene, zumindest bei der Tatsache Buslinien, ein Transfer von der virtuellen Welt in die reale Welt stattgefunden hat. Bei dem Beispiel Buslinien kam es bei Farina auch auf der metaphorischen Ebene zu einem Transfer: „Wenn eine Buslinie in meiner Stadt jetzt überlastet ist, dann denke ich bestimmt, ah, das ist ja genauso. "Auf der dynamischen Ebene ist es bei Farina zu keinem Transfer gekommen, sie kann sich dies aber durchaus vorstellen:

D.K.: $\quad$ Glaubst Du, dass sich aufgrund des Spiels Deine Einstellungen zum Thema intakte Umwelt verändert haben?

Farina: Glaube ich nicht, es war schon vorher so, für mich jetzt. Ich fahr sowieso immer mit dem Bus, weil meine Mutter gegen Autofahren ist. Es ist ja klar, dass viel Autoverkehr schlecht ist und man besser Bahn oder Bus fährt. Wenn einem das vorher nicht klar war, dann vielleicht schon.

Da die Skript- und die Print-Ebene bei diesem Spiel nicht angesprochen wurden, kam es auch zu keinem Transfer auf diesen Ebenen. Auf den anderen drei Ebenen ist es zu einem Transfer gekommen bzw. ist dieser für Farina vorstellbar. Vor diesem Hintergrund muss sehr genau geprüft werden, insbesondere auf der Fakt-Ebene, ob und inwieweit Computersimulationsmodelle tatsächlich in der Lage sind, angemessene Informationen über die reale Welt zu vermitteln.

Dies leitet uns weiter zu dem Aspekt der Räumlichkeit, den ich am Ende unseres Gespräches angesprochen habe:

D.K.: Welche Rolle spielte für Dich der Raum bzw. die räumliche Distanz, wenn z.B. bei bestimmten Flächen Parkplätze ausgewiesen werden müssen?

Farina: Ja, es ist ja schon so, dass man Industriegebiete vier Felder von Wohngebieten weg machen muss. Es spielt schon eine Rolle, wie weit etwas voneinander weg 
ist. Dass ein Parkplatz nicht direkt im Wohngebiet liegt, sondern dass er neben einem Einkaufszentrum liegt, dort wird er ja auch genutzt.

D.K.: $\quad$ Was würdest Du generell zu der Übertragung zwischen Computerspiel und Wirklichkeit sagen?

Farina: Das ist ja gar nicht so wie bei uns! Bei uns werden die Stadtbahnen auf der Straße gebaut und wenn ich da eine Stadtbahn bauen will, kann ich nicht im nachhinein eine Stadtbahn auf die Straße setzen, geht nicht. Teilweise sind die Sachen auch wie bei uns, andererseits denke ich aber auch, dass die Stadt so nicht funktioniert, dass dort jemand sitzt und einfach baut. Das geht ja auch von der Initiative der Bevölkerung aus, wo die was haben wollen. Und nicht einer alleine. Im Spiel ist der Spieler der Stadtplaner und der bestimmt, wo was passiert. Die Stadtbewohner teilen nur mit, ob sie es gut oder schlecht finden, und nicht, dass sie sagen, wir wollen es aber so und so.

Bei der Analyse der Räumlichkeit in der Handlungswelt von Mobility habe ich oben gezeigt, dass Mobility nur zwei Perspektiven auf den Raum berücksichtigt. Dies spiegelt sich auch in diesem Gesprächsausschnitt wider. Bei Mobility werden nur die Perspektiven Containerraum und Raum als System von Lagebeziehungen materieller Orte verwendet. Für Farina ist es jedoch erkennbar, dass so einfach „,die Stadt nicht funktioniert". Sie stellt heraus, dass die realitätsnahe Behandlung der Thematik Mobilität und Stadtplanung nur unter der Berücksichtigung von Initiativen und Mitbestimmung der Bevölkerung möglich ist. Indirekt spricht sie hiermit die im Spiel fehlenden Modelle von Raum als Wahrnehmungskategorie und als soziale Herstellungsleistung an.

Damit ein Unterricht mit Computersimulationen die Lebenswirklichkeit möglichst umfassend aufgreift, müssen einige Aspekte berücksichtigt werden, auf die ich abschließend eingehen werde.

\section{TRÜGERISCHER UND DIDAKTISCHER SCHEIN}

„Es gibt zweierlei Schein - und der Unterschied liegt in der Absicht. Der eine Schein will, dass ich mit ihm rechne, will, dass ich den Abstand zwischen ihm und der Wirklichkeit denke; der andere Schein will, dass ich ihn für die Wirklichkeit halte (...) Was auf dem Bildschirm erscheint, kann fast immer beides sein: einerseits ein Mittel zur besseren und schnelleren Wiedergabe, Veranschaulichung und Gestaltung potentieller Wirklichkeit, andererseits Wirklichkeitsersatz" (von Hentig 2000, S. 28f.)

Mit diesem Zitat von Hartmut von Hentig wird die Problematik der digitalen virtuellen Welten im Allgemeinen angesprochen. Für mein Beispiel der Computersimulation im Geographieunterricht bedeutet dies, dass die Probleme dann auftauchen, wenn vermeintlich ,falsche“ Aspekte der Computersimulation ihren Weg zurück in die Wirklichkeit finden. Dass es tatsächlich zu einem Transfer zwischen virtueller und realer Welt auf unterschiedlichen Ebenen kommen kann, habe ich an dem Beispiel der Schülerin Farina aufgezeigt. Aus diesem Grund ist 
es im Einzelfall zwingend erforderlich zu prüfen, ob und inwieweit das verwendete Computersimulationsmodell tatsächlich in der Lage ist, angemessene Informationen über die Wirklichkeit zu vermitteln. Notfalls muss der (die) Lehrer(in) durch eine korrektive Reflexion eingreifen. Bei Mobility ist dies zum Beispiel bei der funktionalen Entmischung der Fall. Durch die vorgegebene Entmischung der Funktionen Wohnen, Arbeiten, sich Versorgen und Freizeit werden im Spielgeschehen gerade die Probleme geschaffen, mit denen die Städte heutzutage zu kämpfen haben: Separierte und monostrukturierte Nutzungseinheiten, die nicht nur die Erlebnisvielfalt städtischer Räume reduzieren, sondern auch für eine $\mathrm{Zu}$ nahme des motorisierten Individual- und Güterverkehrs sorgen (vgl. BfLR 1996).

Für einen Geographieunterricht, der sich den aktuellen Standards nicht verschließt und der die Wirklichkeit nicht nur reduzierend darstellt, sondern den aktuellen gesellschaftlichen Problemen angemessen gegenübertritt, muss im Falle der Simulation Mobility aber auch gefragt werden: Was geschieht mit den von der Computersimulation gar nicht erfassten Perspektiven auf Raum (hier: „Raum als Kategorie der Sinneswahrnehmung" und „Raum als Resultat von Handlung und Kommunikation")? Wissen die Schüler(innen), dass sie ebenso relevant wie die im Spiel vorkommenden Raummodelle sind? Können sie auf eine andere Weise als durch die Simulation in das Unterrichtsgeschehen eingebunden werden und, wenn ja, wie? Ich selbst habe die Erfahrung gemacht, dass dies jederzeit mit Hilfe eines regionalen Zeitungsartikels zum Thema Stadtentwicklung oder Mobilität möglich ist. Denn hier werden i.d.R. immer die Sichtweisen, Strategien und Entscheidungen unterschiedlicher Akteure sowie ihre strukturellen Handlungsbedingungen thematisiert.

Die Fähigkeit, die virtuelle Welt nicht mit der realen Welt zu vermischen bzw. geleistete Transfer von der virtuellen in die reale Welt richtig einzuordnen und zu bewerten, bezeichnet Fritz als Rahmenkompetenz (Fritz 1997, S. 245). Bei Schüler(inne)n ist diese Rahmenkompetenz eventuell noch nicht voll entfaltet, „,daher ist es sinnvoll, Grenzen zwischen den Welten zu ziehen und den Transfer zwischen ihnen zu kontrollieren“ (Fritz, 1997, S. 245). Für den Einsatz von Computersimulationen im Unterricht bedeutet dies, dass ebenso wie bei der Untat von Steinhäuser Konkurrenzkampf und Karriere, Schützenverein und Schulverweis mitbedacht werden sollten, dass auch im Unterricht nie das alltägliche Leben und alltägliche Erfahrungen aus dem Blick geraten dürfen.

\section{LITERATUR}

Arbeitsgruppe Curriculum 2000+ der Deutschen Gesellschaft für Geographie (2002): Curriculum $2000+$. Grundsätze und Empfehlungen für die Lehrplanarbeit im Schulfach Geographie, in: Geographie Heute, Nr. 200, S. 4-7.

Bundesforschungsanstalt für Landeskunde und Raumordnung (BfLR) (Hrsg.) (1996): Nachhaltige Stadtentwicklung. Herausforderungen an einen ressourcenschonenden und umweltverträglichen Städtebau. Städtebaulicher Bericht, Bonn. 
Fritz, Jürgen (1997): Zwischen Transfer und Transformation. Überlegungen zu einem Wirkungsmodell der virtuellen Welt, in: ders. und Wolfgang Fehr (Hrsg.): Handbuch Medien: Computerspiele. Theorie, Forschung, Praxis, Bonn, S. 229-246.

Funken, Christiane und Martina Löw (2002): Ego-Shooters Container. Raumkonstruktionen im elektronischen Netz, in: Maresch, Rudolf und Niels Werber (Hrsg.): Raum - Wissen Macht, Frankfurt a.M., S. 69-91.

Hemmer, Ingrid (1997): Computersimulationen im Geographieunterricht, in: Schrettenbrunner, Helmut (Hrsg.): Software für den Geographieunterricht (4. Auflage, Neubearbeitung), Nümberg, S. 215-223.

Hentig, Hartmut von (2000): Kolumnen, Stuttgart.

Hülsmann, Thorsten (2000): Geographien des Cyberspace. Wahmehmungsgeographische Studien 19 , Oldenburg.

Krempl, Stefan (2002): Mord ist Sport im Spiel. Interview mit dem Spieleforscher Jürgen Fritz, der eine alleinige Kausalität zwischen Ego-Shootern und Amokläufern als verfehlt betrachtet, in: Telepolis. Magazin der Netzkultur, online: www.telepolis.de

Schroer, Markus (2001): Land und (Daten-)Meer. Zur Besetzung von Räumen im Internet, in: Telepolis. Magazin der Netzkultur, online: www.telepolis.de

www.mobility-online.de. 
Anhang 



\section{AUTORINNEN UND AUTOREN}
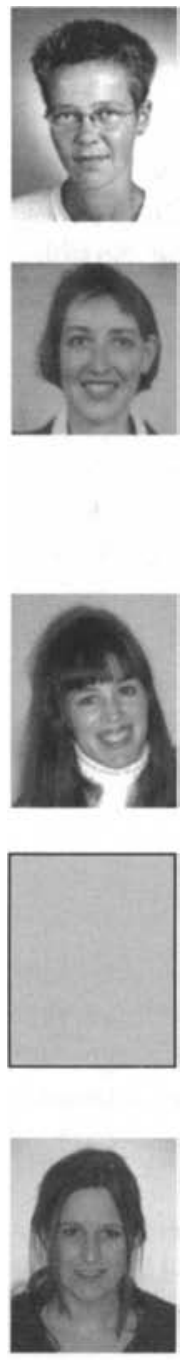

Holger Floeting, Dipl.-Geograph, geb. 1964, wissenschaftlicher Angestellter und Projektleiter am Deutschen Institut für Urbanistik (Difu). Arbeitsgebiete: Informations- und Kommunikationstechnik, Kommunikations- und Medienwirtschaft, kommunale Wirtschaftsförderung, wirtschaftlicher Strukturwandel.

E-Mail: floeting@difu.de

Inga Heinze, Dipl.-Geographin, geb. 1974, Online Producer bei der 1\&1 Internet AG. Arbeitsgebiete: Cybergeography und Interface Design.

E-Mail: inga@cybergeography.de

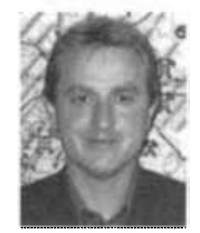

Detlef Kanwischer, Dipl.-Geograph, geb. 1965, wissenschaftlicher Mitarbeiter am Institut für Geographie, Friedrich-Schiller-Universität Jena. Arbeitsgebiete: Geographiedidaktik, Medienpädagogik, Lehrerforschung.

E-Mail: detlef.kanwischer@geogr.uni-jena.de 


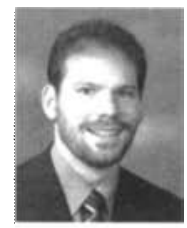

Christian Langhagen-Rohrbach, Dr. phil., geb. 1973, wissenschaftlicher Mitarbeiter am Institut für Kulturgeographie, Stadtund Regionalforschung der Johann Wolfgang Goethe-Universität Frankfurt am Main. Arbeitsgebiete: Stadt- und Regionalforschung, Geographien des Internet.

E-Mail: rohrbach@em.uni-frankfurt.de

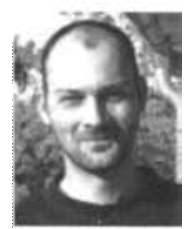

Hubertus Niedermaier, Dipl.-Soziologe, geb. 1973, wissenschaftlicher Mitarbeiter am Institut für Soziologie der Ludwig-Maximilians-Universität München. Arbeitsgebiete: Politische Soziologie, Soziologie des Internet.

E-Mail: niedermaier@soziologie.uni-muenchen.de

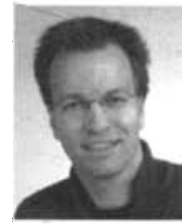

Andreas Pott, Dr. phil., geb. 1968, wissenschaftlicher Mitarbeiter am Fachbereich Gesellschaftswissenschaften der Johann Wolfgang Goethe-Universität Frankfurt am Main. Arbeitsgebiete: Gesellschaft und Raum, Migrationsforschung, Stadtforschung, Tourismusforschung.

E-Mail: pott@soz.uni-frankfurt.de

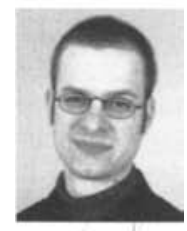

Arne Schlechter, Dipl.-Ingenieur (Raumplanung), geb. 1977, freischaffend. Arbeitsgebiete: Ästhetik \& Städtebau.

E-Mail: a.schlechter@gmx.de

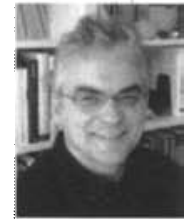

Markus Schroer, Dr. phil., geb. 1964, wissenschaftlicher Assistent am Institut für Soziologie der Ludwig-Maximilians-Universität München. Arbeitsgebiete: Allgemeine Soziologie, Soziologie der Politik, Kultursoziologie, Raumsoziologie, Soziologie des Internet. E-Mail: markus.schroer@soziologie.uni-muenchen.de

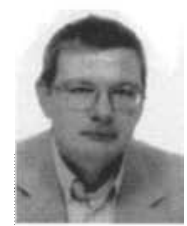

Christian Stegbauer, PD Dr. phil., geb. 1960, Fachbereich Gesellschaftswissenschaften, Johann Wolfgang Goethe-Universität Frankfurt am Main. Arbeitsgebiete: Kommunikationssoziologie; Methoden und Theorien sozialer Netzwerke.

E-Mail: stegbauer@soz.uni-frankfurt.de

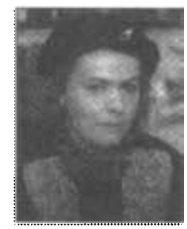

Sabine Thabe, PD Dr. rer. pol., geb. 1962, Fakultät Raumplanung, Universität Dortmund, z.Zt. in Wien. Arbeitsgebiete: Soziologie der Raumplanung; Gesellschaftstheorie \& Ästhetik; Wissenschaftstheorie.

E-Mail: sabine.thabe@chello.at 
Niels Werber, Dr. phil., geb. 1965, Privatdozent für Neuere deutsche Literatur- und Medienwissenschaften an der Ruhr-Universität Bochum. Arbeitsgebiete: Medien der Literatur, Raum und Technik, Evolutionsgeschichte der Literatur, Systemtheorie.

E-Mail: niels.werber@ruhr-uni-bochum.de 



\section{GLOSSAR}

Backbone - (wörtl. Übersetzung: Rückgrat) Bezeichnung für Datenleitungen mit sehr hoher Bandbreite (oft über 10 Gigabit/Sekunde), die die Hauptstrecken der Datennetze bilden. Auf Grund ihrer ,tragenden“ Bedeutung für den Datenverkehr werden sie - analog zur Bedeutung des Rückgrats für den Menschen - als „Backbone“ oder „Backbone-Netze“ bezeichnet.

Bandbreite - Übertragungskapazität einer Datenleitung; wird angegeben in Kilobit, Megabit oder Gigabit pro Sekunde.

Bits pro Einwohner - Einheit, in der die Menge der über das Internet übertragenen Daten pro Einwohner angegeben wird.

Breitband/Broadband - Datenleitung mit hoher Übertragungskapazität; ist der Anschluss eines Endkunden gemeint, so wird der Begriff des Breitbandanschlusses analog zu DSL-Anschluß verwendet. Üblich ist hier eine Bandbreite von ca. $800 \mathrm{Kilobit} /$ Sekunde (im Download).

Country code Top Level Domain (ccTLD) - siehe Top Level Domain.

Domain - Teil des URL, der den Namen des Servers im Internet bestimmt, auf dem sich eine Ressource befindet.

DSL - „Digital Subscriber Line“ (wörtl. Übersetzung: digitale Anschlußleitung); bezeichnet Techniken, mit denen Endkunden digitale Telekommunikationsanschlüsse zusätzlich zum vorhandenen Telefonanschluss zur Verfügung gestellt werden. Bekanntestes Produkt in diesem Segment ist „T-DSL“ der Deutschen Telekom AG.

Genric Top Level Domain (gTLD) - siehe Top Level Domain.

Host - Im allgemeinen Sinne ein Computer, der an eines oder mehrere Netzwerke angeschlossen ist und der damit einen Netzwerkknoten bildet. Im Zusammenhang des World Wide Webs wird ein Rechner dann als Host bezeichnet, wenn auf ihm Webseiten abgelegt sind und diese von Benutzern des WWW abgerufen werden können.

Hub - Nach Castells ein Umschlagplatz für Informationen, Kapital und Innovationen in der Hierarchie der Weltstädte bzw. abstrakter im "space of flows“ (im Gegensatz zu „nodes“, die Knoten im Sinne von Knoten in Daten- oder Verkehrsnetzen bezeichnen).

Internettraffic - Menge der im gesamten Internet oder in Teilbereichen des Netzes übertragenen Daten.

ISP - „Internet Service Provider“; Unternehmen, das einer Firma oder einer Privatperson die Nutzung des Internet ermöglicht, zum einen, indem ein Internetanschluss zur Verfügung gestellt wird, zum anderen, indem ein Server für eigene Angebote des Kunden vorgehalten wird.

IP - „Internet Protocol“; Übertragungsprotokoll, das die Datenübertragung im Internet regelt. Damit verbunden ist auch die Vergabe sog. IP-Adressen, die aus vier Zahlenkolonnen bestehen. Jede Zahlenkolonne kann einen Wert von 0 bis 255 annehmen und ermöglicht die sichere Identifikation eines an das Internet angeschlossenen Computers. Eine IP-Adresse kann z.B. 192.168.0.1 lauten. 
MP3 - Von den deutschen Fraunhofer-Instituten entwickeltes Verfahren zur Kompression von Musikdateien.

Multi User Domain - virtuelle Umgebung für Rollenspiele im Internet.

Router - Gerät, das bei der Datenübertragung im Internet den nächsten Punkt im Netzwerk bestimmt, an den ein Datenpaket weitergeleitet wird.

Server - Computer, der Anwendungen und Dokumente bereithält, auf die andere Computer zugreifen können.

Top Level Domain (TLD) - der allgemeine Teil eines Domainnamens in einer Internet-Adresse. Eine TLD kann entweder eine generic top-level domain (gTLD), wie z.B. .com, .org oder .edu, sein oder eine regional spezifische country code top-level domain (ccTLD), wie z.B. ,.de“ für Deutschland.

Traceroute-Programme - Software, mit deren Hilfe die Übertragungsstationen eines Datenpaketes, das über das Internet verschickt wird, ermittelt werden können. Für Webseiten gibt der URL typischerweise das HTTP-Protokoll, d.h. den Hostnamen des Servers, auf dem die Seite liegt, und den Dateipfad zur entsprechenden Seite, an

(Bsp.: http://www.cybergeography.de/index.htm).

Uniform Resource Locator (URL) - dient der Lokalisierung von Ressourcen im Internet mittels einer Zeichenfolge mit standardisierter Syntax.

Usenet/Newsgroups - Eigener Internetdienst für Online-Diskussionen. Die Benennung erfolgt ähnlich den Domain-Namen. So lautet der Name einer deutschen Newsgroup zum Thema PC-Monitore bspw. de.comp.hardware. monitore. Gelesen werden können Newsgroups mit Newsreadern, wie sie z.B. in Microsoft Outlook integriert sind. Online-Dienste wie google. com verfügen über umfangreiche Archive der in den Newsgroups veröffentlichten Beiträge.

W3B - Internetnutzeranalyse der Hamburger Fa. Fittkau \& Maaß; W3B ist die Abkürzung für WWW-Benutzeranalyse.

WHOIS - Dienst, der es erlaubt, personenbezogene Daten wie Mailadressen, Postadressen, Telefon- und Telefaxnummern von Domaininhabern zu ermitteln.

WWW - „Worldwide Web“; Internetdienst, in dem mit Hilfe der Sprache „HTML“ (Hypertext Markup Language) Seiten in sog. Browsern (Netscape, Opera, Internet Explorer) dargestellt werden. 
Dieser Band führt zwei interdisziplinäre Forschungsfelder zusammen: Die Untersuchung der gesellschaftlichen Bedeutung des Internets und die theoretische Diskussion zum Verhältnis von Raum und Gesellschaft. Die räumlichen und gesellschaftlichen Dimensionen des Internets werden anhand physisch-materieller, struktureller, perzeptiver und kommunikativer Aspekte analysiert. Sichtbar wird dabei nicht zuletzt, welche Impulse die Raumdebatte am Forschungsobjekt Internet gewinnen kann.

Der erste Teil des Bandes behandelt die Problematik der Verortung des Internets. Der zweite Teil identifiziert auf zweierlei Weise einen strukturgenerierenden Geocode des Internets. Der eingehenden Bestimmung und Untersuchung verschiedenartiger Räume des Internets widmet sich der dritte Teil des Bandes. 